UCRL-LR-105090

Distribution Category UC-705

UCRL-LR- - 105090

DE91 002566

\title{
A Numerical Theory of Lattice Gas and Lattice Boltzmann Methods in the Computation of Solutions to Nonlinear Advective-Diffusive Systems
}

Altamont Bracy Hamilton Elton (Ph.D. Thesis)

Manuscript date: September 24, 1990

\section{LAWRENCE LIVERMORE NATIONAL LABORATORY University of California - Livermore, California • 94551}

Available to DOE and DOE contractors from the Office of Scientific and Technical Information P.O. Box 62, Oak Ridge, TN 37831 Prices available from (615) 576-8601, FTS 626-8401

Available from: National Technical Information Service - U.S. Department of Commerce 5285 Port Royal Road • Springfield, VA $22161 \cdot$ A14 $\bullet$ (Microfiche A01) 


\section{A NUMERICAL THEORY OF \\ LATTICE GAS AND LATTICE BOLTZMANN METHODS \\ IN THE COMPUTATION OF SOLUTIONS TO \\ NONLINEAR ADVECTIVE-DIFFUSIVE SYSTEMS}

\section{By}

ALTAMONT BRACY HAMILTON ELTON

B.S. Cum laude (Pacific Lutheran University) 1983 ,

M.S. (University of California, Davis) 1985

\section{DISSERTATION}

Submitted in partial satisfaction of the requirements for the degree of DOCTOR OF PHILOSOPHY

in

Computer Science

in the

GRADUATE DIVISION

of the

UNIVERSITY OF CALIFORNIA

DAVIS

Approved:

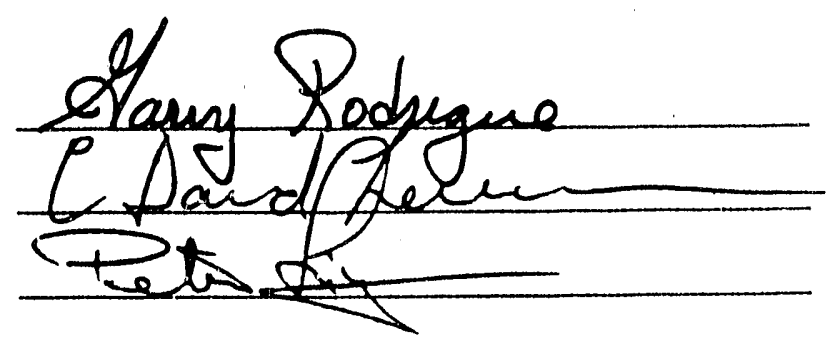

Committee in Charge 


\title{
A Numerical Theory of \\ Lattice Gas and Lattice Boltzmann Methods \\ in the Computation of Solutions to \\ Nonlinear Advective-Diffusive Systems
}

\author{
Altamont Bracy Hamilton Elton \\ Department of Applied Science \\ University of California, Davis \\ Dissertation Committee: \\ Professor Garry H. Rodrigue, Chair (University of California, Davis) \\ Professor C. David Levermore (University of Arizona, Tucson) \\ Professor Peter Linz (University of California, Davis)
}

\begin{abstract}
A numerical theory for the massively parallel lattice gas and lattice Boltzmann methods for computing solutions to nonlinear advective-diffusive systems is introduced. The convergence theory is based on consistency and stability arguments that are supported by the discrete Chapman-Enskog expansion (for consistency) and conditions of monotonicity (in establishing stability).

The theory is applied to four lattice methods: Two of the methods are for some two-dimensional nonlinear diffusion equations. One of the methods is for the onedimensional lattice method of [B. Boghosian and C. D. Levermore, Complex Systems 1(1):1987, pp. 17-30] for the one-dimensional viscous Burgers equation. And one of the methods is for a two-dimensional nonlinear advection-diffusion equation. Conve1gence is formally proven in the $L_{1}$-norm for the first three methods, revealing that they are second-order, conservative, conditionally monotone finite difference methods. Computational results which support the theory for lattice nethods are presented.

In addition, a domain decomposition strategy using mesh refinement techniques is presented for lattice gas and lattice Boltzmann methods. The strategy allows concentration of computational resources on regions of high activity. Computational evidence is reported for the strategy applied to the lattice gas method for the onedimensional viscous Burgers equation.
\end{abstract}




\section{Preface}

Since around 1986, when lattice gas methods were first seen in terms of cellular automata [72], there has been tremendous interest in the methods as they apply to hydrodynamics. Much of the interest has resulted in numerous applications and new methods. As early as $1987-8$, the Lattice Boltzmann Approximation to lattice gas methods was being applied as a finite difference technique. Throughout these developments, little headway has been made regarding the numerical stature of the methods. While the main purpose of the dissertation is to introduce a numerical theory for lattice gas and lattice Boltzmann methods, a secondary purpose is to help foster further numerical investigations of the methods. I hope that this dissertation will serve well in this secondary capacity, eliciting a growing interest in lattice methods toward a greater understanding of their numerical and computational relevance.

I would like to express my gratitude to those individuals and institutions that helped make this work possible. I thank Professor Garry Rodrigue for patiently guiding me through to completion of this work. I learned from him the basic tools of research in numerical analysis and advanced scientific computing. He also taught me how to prepare and review papers and to deliver presentations. I thank him most for enthusiastically revealing to me the wonderful world of computational sciences.

To Professor C. David Levermore, I am indebted for all that he taught me regarding the technical aspects of lattice methoris and the mathematics of partial differential 
equations. I appreciate his steadfast patience in working with me. I thank Dr. Gerry Hedstrom for helping me with solutions of partial differential equations. I also thank Dr. Bruce Boghosian, who helped me with some questions about the discrete Chapman-Enskog procedure. I appreciate the comments and criticism of Professor Peter Linz. To Professor Michael Dollinger I owe many thanks, for he first taught me about communicating mathematics. I thank Professors John Killeen, Garry Rodrigue, Gene Fisher, Rao Vemuri, and Fred Wooten for serving on my Qualifying Committee. I am grateful to Russell Brand, Dr. Gary Doolen, Dr. Farid Dowla, Dr. Mark Durst, Carolyn Hunt, Dr. Philip Manwell, Abigail Staley, Chuck Moore, Matt Nolan, Dr. Jeffrey Scroggs, Dr. Mark Seager, Clement Ulrich, and other friends and colleagues for their input and support. I also thank the secretarial staff of the Department of Applied Science. Finally, I appreciate the enduring support of each of my parents.

I am glad to have been a student in the last course sequence taught by Frofessor John Killeen. In a project for one of those courses, which were in computational physics, I first began to study seriously using the Lattice Boltzmann Approximation as a finite difference technique, i.e., the lattice Boltzmann method. He had said that the project might serve in some way as a foundation for the dissertation. Despite my doubts that it would serve for me in such a capacity, that project helped focus my efforts on lattice Boltzmann methods, which constitutes the major subject of the dissertation. In the courses, I learned about the many techniques used in computational physics, especially as they relate to solving partial differential equations of plasma physics. It became clear that better methods are vital to increased understanding in this and related areas. I thank Professor Killeen for instilling this in me. 
I acknowledge ard greatly appreciate the financial support of the Lawrence Livermore National Laboratory (LLNL) through its Student Employee Program. I thank the following for the use and availability of their respective computer resources:

- the Computing and Mathematics Research Division, LLNL,

- the Mathematics and Computer Science Division, Argonne National Laboratory,

- the National Energy Research Supercomputer Center, LLNL.

This dissertation was typeset in $\mathrm{IAT}_{\mathrm{E}} \mathrm{X}$ [51] with Psfig/ $\mathrm{T}_{\mathrm{E}} \mathrm{X}$ [29] under the support of the $\mathrm{VOR}_{\mathrm{O}} \mathrm{T}_{\mathrm{E}} \mathrm{X}$ document preparation system [19-22].

Livermore, California

Bracy H. Elton

September 1990 


\section{To Professor John Killeen}

\section{Director}

National Energy Supercomputer Research Center Lawrence Livermore National Laboratory

and

Associate Dean

Department of Applied Science

University of California, Davis

In the spirit toward new methods for computational physics.

And in memory of my grandparents,

Dr. and Mrs. Norman William Elton

and

Mr. and Mrs. Altamont Bracy Hamilton, Jr. 


\section{Contents}

Abstract $\quad$ i

Preface iii

Notation $\quad$ xiii

List of Tables $\quad$ Xv

List of Figures xvii

List of Definitions, Lemmas, Theorems, and Corollaries xix

List of Equations $\quad$ xxiii

1 Introduction 1

2 Theory 7

2.1 Definitions and Notation $\ldots \ldots \ldots \ldots \ldots$

2.1 .1 Basic Definitions . . . . . . . . . . . . . . 8

2.1.2 Lattice Gas Methods . . . . . . . . . . . . . . . . . . . . . 14

2.1.3 Lattice Boltzmann Methods . . . . . . . . . . . . . . 31

2.2 Equilibrium Analysis . . . . . . . . . . . . . . . . . 35

2.2.1 Determining Equilibria Directly . . . . . . . . . . 36

2.2 .2 Equilibrium Theorem . . . . . . . . . . . . . . . . . 36

2.2.3 Applying the Equilibrium Theorem . . . . . . . . . . . . 41

2.3 Discrete Chapman-Enskog Analysis . . . . . . . . . . . . . 44

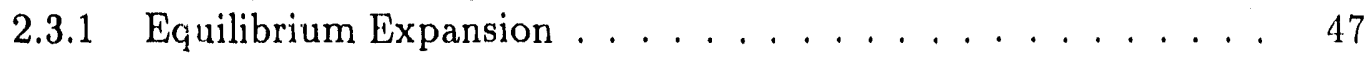

2.3.2 Linearized Collision Operator . . . . . . . . . . . . . . 48

2.3.3 Collision Operator Expansion _. . . . . . . . . . . . 58

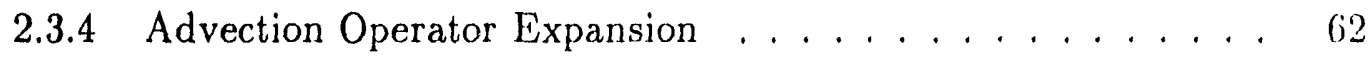

2.3.5 Matching the Expansions . . . . . . . . . . . . . . 67

2.4 Convergence Analysis . . . . . . . . . . . . . . . . . . . . . 82 
2.4 .1 Definitions . . . . . . . . . . . . . . . . . . . 84

2.4 .2 Consistency . . . . . . . . . . . . . . . . . 88

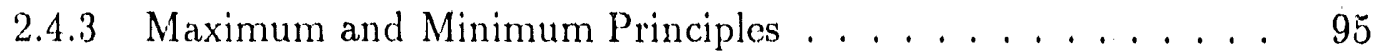

2.4 .4 Stability . . . . . . . . . . . . . . . . 113

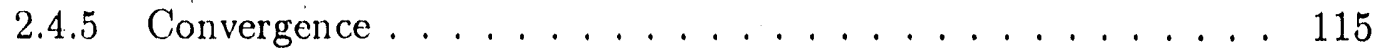

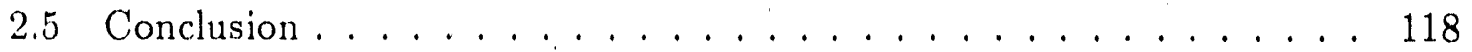

3 Applications 121

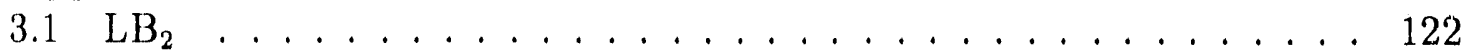

3.1 .1 Collision Rules . . . . . . . . . . . . . . . . . . . 122

3.1 .2 Collision Operator . . . . . . . . . . . . . . . 125

3.1.3 Lattice Boltzmann Approximation . . . . . . . . . . . 125

3.1 .4 Equilibrium Analysis . . . . . . . . . . . . . . . 125

3.1.5 Discrete Chapman-Enskog Expansion . . . . . . . . . . 127

3.1 .6 Convergence . . . . . . . . . . . . . . . . . . 137

3.2 A Lattice Method for the 1-D Viscous Burgers Equation . . . . . . 154

3.2 .1 Collision Rules . . . . . . . . . . . . . . . . . . 155

3.2 .2 Collision Operator . . . . . . . . . . . . . . 155

3.2.3 Lattice Boltzmann Approximation . . . . . . . . . . . 157

3.2 .4 Equilibrium Analysis . . . . . . . . . . . . . . . . . 158

3.2 .5 Linearized Collision Operator . . . . . . . . . . . . 159

3.2 .6 Eigenvalues anià Eigenvectors . . . . . . . . . . . . . . 159

3.2.7 Discrete Chapman-Enskog Expansion . . . . . . . . . . . . 160

3.2 .8 Convergence . . . . . . . . . . . . . . . . . . . . . 170

3.3 A Lattice Method for a 2-D Advection-Diffusion Equation . . . . . 180

3.3.1 Collision Rules . . . . . . . . . . . . . . . . . . . . . 181

3.3.2 Collision Operator . . . . . . . . . . . . . . . . . . 181

3.3.3 Lattice Boltzmann Approximation . . . . . . . . . . . . . 185

3.3 .4 Equilibrium Analysis . . . . . . . . . . . . . . . 186

3.3.5 Linearized Collision Operator . . . . . . . . . . . . . 187

3.3 .6 Eigenvalues and Eigenvectors _. . . . . . . . . . . . 188

3.3.7 Discrete Chapman-Enskog Expansion . . . . . . . . . . . . 188

3.4 Conclusions . . . . . . . . . . . . . . . . . . . . . . . 192

4 Computational Studies 195

$4.1 \quad \mathrm{LB}_{1} \ldots \ldots \ldots \ldots \ldots \ldots \ldots \ldots \ldots \ldots \ldots$

4.1.1 Finite Difference Method . . . . . . . . . . . . . . . 197

4.1 .2 Numerical Results . . . . . . . . . . . . . . . . . . 198

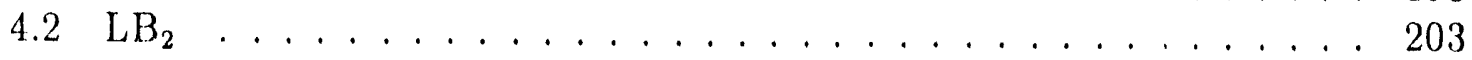

4.2 .1 Finite Difference Method . . . . . . . . . . . . . . . . . 203

4.2 .2 Numerical Results . . . . . . . . . . . . . . . . . . . 203 
4.3 1-D Burgers' Equation LB Method . . . . . . . . . . . . . . . . 209

4.3.1 Finite Difference Method . . . . . . . . . . . . . . . . . . 209

4.3.2 Numerical Results . . . . . . . . . . . . . . . . . . 210

4.4 2-D Advection-Diffusion LB Method . . . . . . . . . . . . . . . 214

4.4.1 Finite Difference Method . . . . . . . . . . . . . . . . . 214

4.4 .2 Numerical Results . . . . . . . . . . . . . . . . . 215

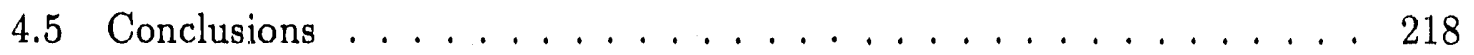

5 A Domain Decomposition for Lattice Methods 221

5.1 A Sub-Structuring Method for a Lattice Gas Method for the 1-D Viscous Burgers Equation . . . . . . . . . . . . . . . . 223

5.2 Computational Evidence . . . . . . . . . . . . . . . . 225

5.3 Conclusions . . . . . . . . . . . . . . . . . 226

6 Conclusions 231

\section{APPENDICES:}

A LB $_{1}$ Analysis Details $\quad 235$

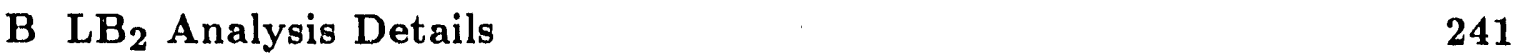

C Convergence of a Nonlinear Finite Difference Scheme 245

C.1 Nonlinear Problem . . . . . . . . . . . . . . . . . . . . 245

C.2 Explicit Finite Difference Method . . . . . . . . . . . . . . 246

C.3 Maximum Principle . . . . . . . . . . . . . . . . . . . . . . . . . . . 246

C.4 Consistency . . . . . . . . . . . . . . . . . . . . 248

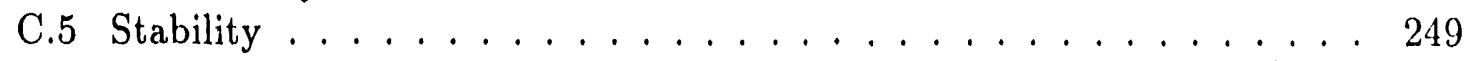

Bibliography 253

$\begin{array}{ll}\text { Index } & 261\end{array}$ 


\section{Notation}

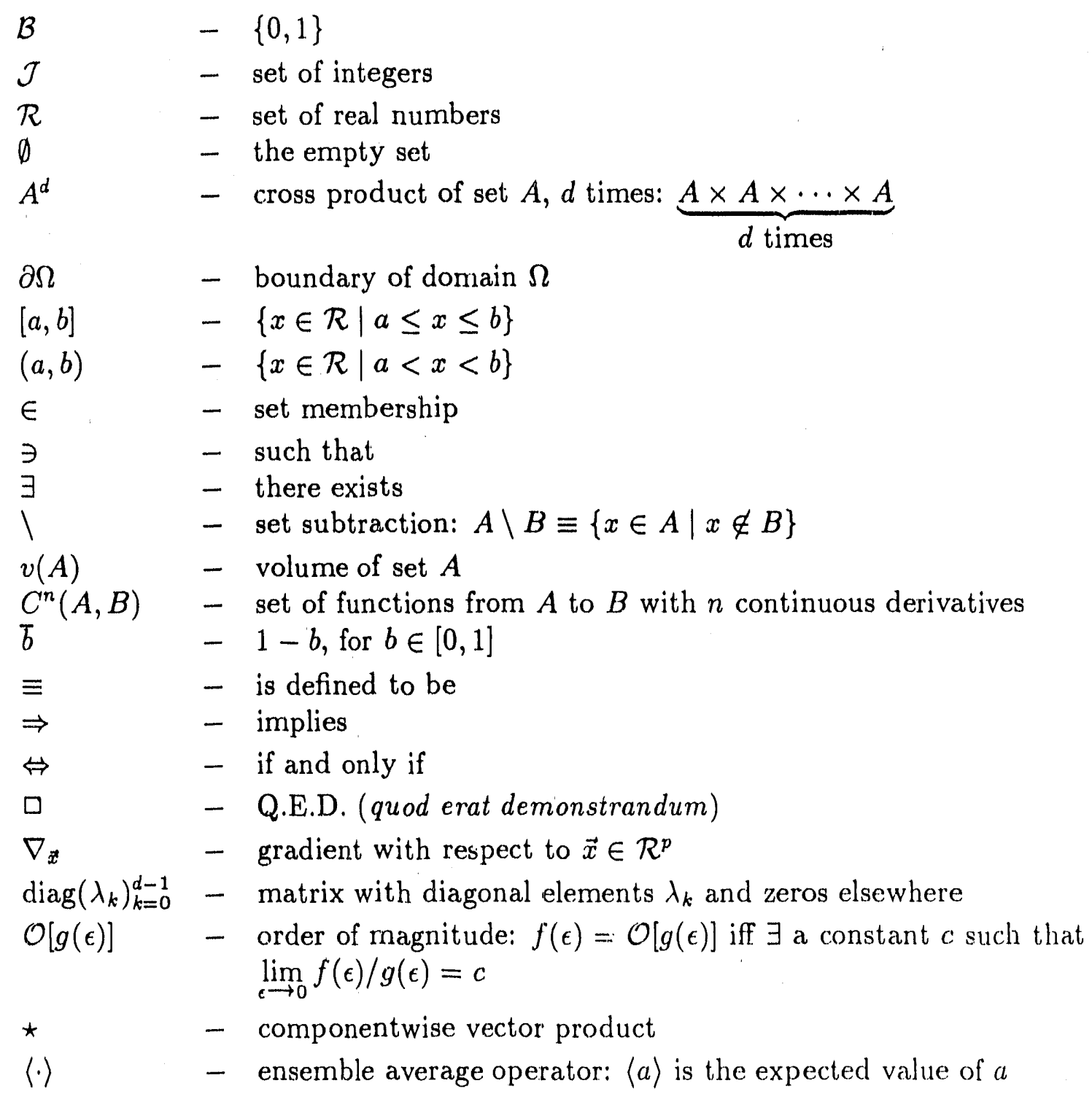



$\delta \quad-\quad-0<\delta \ll 1$, small dimensionless parameter
$T \quad$ - temporal scale length
I $\quad$ - spatial scale length
d. - number of directions on the lattice structure of a lattice method
p - number of spatial dimensions on a lattice
$\Delta x \quad-L \delta$, lattice spacing
$\Delta t \quad-T \delta^{2}$, temporal spacing
k. $\quad-\in\{0,1, \ldots, d-1\}$, lattice direction index
$n-\in \mathcal{J} \ni n \geq 0$, time step index
$\mathfrak{I} \quad-$ a $p$-dimensional Bravais lattice in $\mathcal{R}^{p}$
$\vec{e}_{k} \quad-\quad$ (unit) velocity vector in direction $k$
$\mathfrak{G}$ - crystallographic group of isometries globally preserving the set of velocity vectors

$\vec{\imath} \quad-\in \mathfrak{L}$, position vector on Bravais lattice $\mathfrak{L}$, it is a linear combination with integer coefficients of $p$ generating vectors (see [1])

$\hat{\mathbf{n}}(\vec{\imath} ; n) \quad-\in \mathcal{B}^{d}$, vector of occupation numbers at $(\vec{\imath} ; n)$

$\langle\hat{\mathbf{n}}(\vec{\imath} ; n)\rangle-\epsilon[0,1]^{d}$, ensemble average or expected value of $\hat{\mathbf{n}}(\vec{\imath} ; n)$

$\left(n_{k}\right)_{i}^{n}-\in[0,1]$, mean occupation number in direction $k$ at $(\vec{\imath} ; n)$

$\mathrm{n}(\vec{\imath} ; n) \quad-=\langle\hat{\mathbf{n}}(\vec{\imath} ; n)\rangle \in[0,1]^{d}$, vector of mean occupation numbers at $(\vec{\imath} ; n)$

$\mathbf{n}_{\vec{\imath}}^{n} \quad-\quad=\mathbf{n}(\vec{\imath} ; n)$

$\mathrm{n}(\vec{x} ; t) \quad-\quad=\sum_{j=0}^{\infty} \delta^{j} \mathbf{n}^{(j)}(\vec{x} ; t)$ is an equilibrium expansion about equilibrium $\mathbf{n}^{(0)}$

$\alpha, \beta-\quad-\in \mathcal{B}^{d}$, state at a node in a lattice gas method

$\mathcal{S}(\alpha \rightarrow \beta)-\in[0,1]$, state transition function: the probability of a lattice node in state $\alpha$ changing to state $\beta$ in effecting the collision phase

$\mathcal{D}^{j} \mathcal{C}(\mathbf{n}) \quad-\quad=\frac{\partial^{j}}{\partial \mathbf{n}^{j}} \mathcal{C}(\mathbf{n})$, differential operator (on mean occupation num-

A $\quad$ - advection operator

$\mathcal{C}$ - collision operator

$n^{(0)} \quad-\in[0,1]^{d}$, equilibrium state (ground state in discrete ChapmanEnskog asymptotic expansion)

$\mathcal{L} \quad-\quad=\left.\mathcal{D} \mathcal{C}^{(0)}(\mathbf{n})\right|_{\mathbf{n}=\mathbf{n}^{(0)}}$, linearized collision operator (linearized about equilibrium $\mathrm{n}^{(0)}$ )

$\mathcal{L}^{+} \quad-\quad$ pseudo-inverse of $\mathcal{L}$ 


\section{List of Tables}

$2.1 \quad \mathrm{LB}_{1}$ Collision Rules. . . . . . . . . . . . . . . .

2.2 Collision Rules for an Example of Semi-Detailed Balance without De-

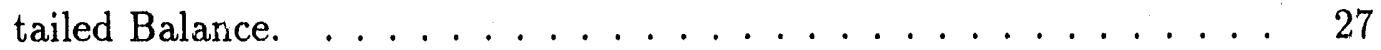

2.3 The Sigi of the First Partial Derivatives of $f(x, y) \ldots \ldots \ldots \ldots$

$2.4 \mathrm{LB}_{1}$ Componentwise Eigenvector Products. . . . . . . . . . 55

2.5 Boundary Extrema of $f(\zeta, \rho, \phi)$ and $g(\zeta, \rho, \phi)$ in $\mathrm{LB}_{1} . \ldots \ldots 106$

2.6 Direction of Increase in $f$ and $g$ for Arguments Just Outside $\mathcal{E}$ in $L_{1} B_{1} .109$

$3.1 \mathrm{JB}_{2}$ Collision Rules. . . . . . . . . . . . . . . . . . . 124

3.2 Boundary Extrema of $f(\zeta, \rho, \phi), g(\zeta, \rho, \phi)$, and $h(\zeta, \rho, \phi)$ in $\mathrm{LB}_{2} \ldots 148$

3.3 Extrema of $H$ in $\mathrm{LB}_{2} \ldots \ldots \ldots \ldots \ldots \ldots \ldots \ldots$

3.4 Direction of 1 increase in $f, g$, and $h$ for Arguments just Outside $\mathcal{E}$ in $\mathrm{LB}_{2} \ldots \ldots \ldots \ldots \ldots \ldots \ldots \ldots \ldots \ldots$

3.5 Collision Rules of a Lattice Met iod for Eq. 3.29. . . . . . . . . . 156

3.6 Componentwise Eigenvector Products. . . . . . . . . . . . . . . 160

3.7 Pictorial Description of Collision Rules for Two-Dimensional AdvectionDiffusion. . . . . . . . . . . . . . . . . . . . . 182

3.8 Collision Rules for Two-Dimensional Advection-Diffrision. . . . . . . 183

4.1 $L_{1}$-norm Comparison of $L B_{1}$ - and Finite Difference-Computed Solutions with Initial Condition Parameters, $A=1 / \sqrt{20}$ and $B=1 / 2$. . 201

$4.2 L_{\infty}$-norm Comparison of $\mathrm{LB}_{1}$ - and Finite Difference-Computed Solutions with Initial Condition Parameters, $A=1 / \sqrt{20}$ and $B=1 / 2$. . 201

4.3 $L_{1}$-norm Comparison of $\mathrm{LB}_{1}$ - and Finite Difference-Computed Solutions with Initial Condition Parameters, $A=0.45$ and $B=1 / 2 . \ldots 202$

4.4 $L_{\infty}$-norm Comparison of $\mathrm{LB}_{1^{-}}$and Finite Difference-Computed Solutions with Initial Condition Parameters, $A=0.45$ and $B=1 / 2 . \ldots 202$

$4.5 L_{1}$-norm Comparison of $\mathrm{LB}_{2^{-}}$and Finite Difference-Computed Solutions with Initial $C$ ndition Parameters, $A=1 / 12$ and $B=3 / 4$. . 207

4.6 $L_{\infty}$-norm Comparison of $\mathrm{LB}_{2^{-}}$and Finite Difference-Computed Solutions with Initial Condition Parameters, $A=1 / 12$ and $B=3 / 4 . \quad \ldots 207$

4.7 $L_{1}$-norm Comparison of $\mathrm{LB}_{2}$ - and Finite Difference-Computed Solutions with Initial Condition Parameters, $A=0.45$ and $B=1 / 2 \ldots 208$ 
4.8 $L_{\infty}$-norm Comparison of $\mathrm{LB}_{2}$ - and Finite Difference-Computed Solutions with Initial Condition Parameters, $A=0.45$ and $B=1 / 2 \ldots 208$

4.9 Comparison of Lattice Boltzmann- and Finite Difference-Computed Solutions to Eq. 3.34 at $t=1 / 4$ with $\nu=2^{-8}$ and Initial Condition $n_{I}(x)=(\cos (2 \pi x)+1) / 2 \ldots \ldots \ldots \ldots \ldots \ldots$

4.10 Comparison of Coarse and Fine Grid Finite Difference Computed Solutions to Eq. 3.34 at $t=1 / 4$ with $\nu=2^{-8}$ and Initial Condition $u_{I}(x)=(\cos (2 \pi x)+1) / 2 \ldots \ldots \ldots \ldots \ldots \ldots$

4.11 Comparison Lattice Boltzmann- and Finite Difference-Computed SoJutions to Eq. 3.58 at $t=1 / 8$ for $\nu=1 / 32$ and Initial Condition $(\cos (2 \pi x)+1) / 2 \ldots \ldots \ldots \ldots \ldots \ldots \ldots \ldots \ldots . \ldots \ldots \ldots$

4.12 Comparison of Lattice Boltzmann- anc Firite Difference-Computed Solutions to Eq. 3.58 at $t=1 / 8$ for $\nu=1 / 16$ and Initial Condition $(\cos (2 \pi x)+1) / 2 . \ldots \ldots \ldots \ldots \ldots \ldots \ldots$

4.13 Comparison of Lattice Boltzmann- and Finite Difference-Computed Solutions to Eq. 3.58 at $t=1 / 8$ for $\nu=1 / 8$ and Initial Condilin $(\cos (2 \pi x)+1) / 2 \ldots \ldots \ldots \ldots \ldots \ldots \ldots \ldots$

4.14 Comparison of Lattice Boltzmann- and Finite Difference-Computed Solutions to Eq. 3.58 at $t=1 / 8$ for $\nu=1 / 32$ and Initial Condition $(\sin (2 \pi x) \sin (2 \pi y)+1) / 2 \ldots \ldots \ldots \ldots \ldots \ldots \ldots$ 


\section{List of Figures}

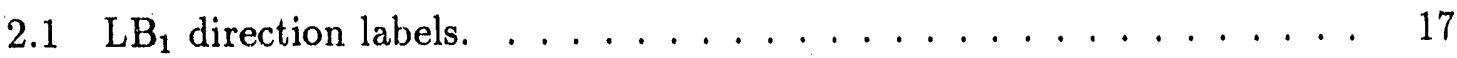

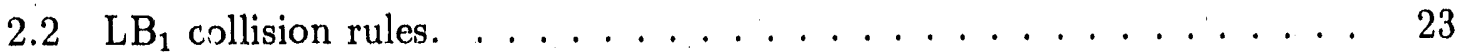

2.3 Graph of $f(x, y)=x \log (x / y)+y-x . \ldots \ldots \ldots \ldots \ldots$

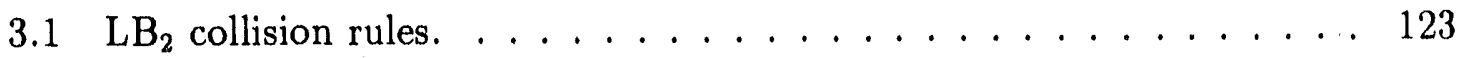

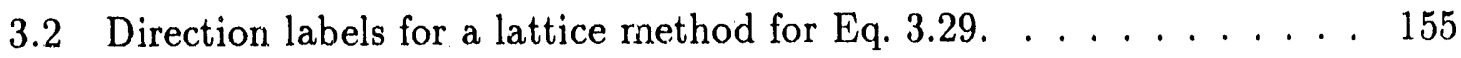

3.3 Collision ruies of a lattice method for the one-dimensional viscous Burgers equation. . . . . . . . . . . . . . 156

4.1 Evolution of $u(x, y ; t)$ according to Eq. 2.29 with $\nu=1 / 2$ : (a) Initial condition, $u(x, y ; 0)=u_{I}(x, y)=A \sin (2 \pi x) \sin (2 \pi y)+B$, where $A=$ $1 / \sqrt{20}$ and $B=1 / 2$; (b) $u(x, y ; t)$ at $t=1 / 32$.

4.2 Evolvtion of $u(x, y ; t)$ according to Eq. 2.29 with $\nu=1 / 2$ : (a) Initial condition, $u(x, y ; 0)=u_{I}(x, y)=A \sin (2 \pi x) \sin (2 \pi y)+B$, where $A=$ 0.45 and $B=1 / 2 ;$ (b) $u(x, y ; t)$ at $t=1 / 32 \ldots \ldots \ldots \ldots \ldots$

4.3 Evolution of $u(x, y ; t)$ according to Eq. 3.6 with $\nu=1 / 2$ : (a) Initial condition, $u(x, y ; 0)=u_{I}(x, y)=A \sin (2 \pi x) \sin (2 \pi y)+B$, where $A=$ $1 / 12$ and $B=3 / 4 ;(\mathrm{b}) u(x, y ; t)$ at $t=1 / 32$.

4.4 Evolution of $u(x, y ; t)$ according to Eq. 3.6 with $\nu=1 / 2$ : (a) Initial condition, $u(x, y ; 0)=u_{I}(x, y)=A \sin (2 \pi x) \sin (2 \pi y)+B$, where $A=$ 0.45 and $B=1 / 2$; (b) $u(x, y ; t)$ at $t=1 / 32$.

4.5 Evolution of $u(x ; t)$ according to Eq. 3.34 with $\nu=2^{-8}$ : (a) Initial condition $u(x ; 0)=u_{I}(x)=(\cos (2 \pi x)+1) / 2 ;(\mathrm{b}) u(x ; t)$ at $t=1 / 4$.

4.6 Comparison of lattice Boltzmann-computed and finite difference-computed solutions to Eq. 3.34 at $t=1 / 4$ with $\nu=2^{-8}$ and varying grid sizes: The finest grid, i.e., $N=32768$, is the finite difference-computed solution, $V(x, t)$; calculations for grid sizes $N \in\{256,192,160,128\}$ are the lattice Boltzmann-computed solutions, $U(x, t) \ldots \ldots \ldots \ldots 212$

4.7 Evolution of $u(x, y ; t)$ according to Eq. 3.51 with $\nu=2^{-5}$ : (a) Initial condition, $u(x, y ; 0)=u_{I}(x, y)=(\cos (2 \pi x)+1) / 2 ;$ (b) $u(x, y ; t)$ at $t=1 / 8$. 
4.8 Evolution of $u(x, y ; t)$ according to Eq. 3.51 with $\nu=2^{-5}$ : (a) Initial condition, $u(x, y ; 0)=u_{I}(x, y)=(\sin (2 \pi x) \sin (2 \pi y)+1) / 2$; (b) $u(x, y ; t)$ at $t=1 / 8 \ldots \ldots \ldots \ldots \ldots \ldots \ldots \ldots \ldots \ldots \ldots$

5.1 Initial condition. . . . . . . . . . . . . . . 227

5.2 (a) Coarse lattice and (b) fine lattice solutions; $t=0.125 ; M=1 . \quad \ldots \quad 227$

5.3 (a) Coarse lattice and (b) fine lattice solutions; $t=0.125 ; M=2 . \quad \ldots \quad 228$

5.4 (a) Coarse lattice and (b) fine lattice solutions; $t=0.125 ; M=4 . \quad$. . 228

5.5 (a) Coarse lattice and (b) fine lattice solutions; $t=0.125 ; M=8$. . . 229 


\section{List of Definitions, Lemmas, Theorems, and Corollaries}

Definition 2.1 Vector Exponentiation $\ldots \ldots \ldots \ldots \ldots$

Definition 2.2 Order of Magnitude . . . . . . . . . . . . . . . . . . . 12

Definition 2.3 Connectedness . . . . . . . . . . . . . . . . . 12

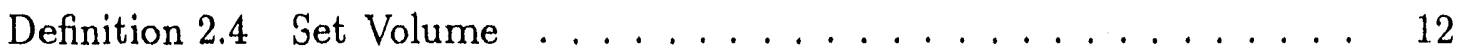

Definition 2.5 Covariance . . . . . . . . . . . . . . . . . 13

Definition 2.6 Fermi Exclusion Principle . . . . . . . . . . . . . . . . 14

Definition 2.7 Lattice . . . . . . . . . . . . . . . . . . . 15

Definition 2.8 Occupation Number . . . . . . . . . . . . . . . . . 16

Definition 2.9 Advection Operator . . . . . . . . . . . . . . . . . 19

Definition 2.10 State Transition Function . . . . . . . . . . . . 20

Definition 2.11 Lattice Isometry Invariance $\ldots \ldots \ldots \ldots$

Definition 2.12 Collision Rules . . . . . . . . . . . . . . . . 21

Definition 2.13 Conserved Quantity . . . . . . . . . . . . . . . 21

Definition 2.14 Mass-Conserving . . . . . . . . . . . . . . . . 24

Definition 2.15 Detailed Balance . . . . . . . . . . . . . . 24

Definition 2.16 Semi-Detailed Balance . . . . . . . . . . . . . 25

Definition 2.17 Quasi-Detailed Balance . . . . . . . . . . . . . . 25

Definition 2.18 Collision Operator . . . . . . . . . . . . . . . . . . . . 28

Definition 2.19 Lattice Gas Method . . . . . . . . . . . . . . . . . . . . 28

Definition 2.20 Rotation Invariance $\ldots \ldots \ldots \ldots$

Definition 2.21 Mean Occupation Numbers . . . . . . . . . . . . . . . . 31

Definition 2.22 Lattice Boltzmann Approximation . . . . . . . . . . . . 32

Definition 2.23 Lattice Boltzmann Method . . . . . . . . . . . . . . 33

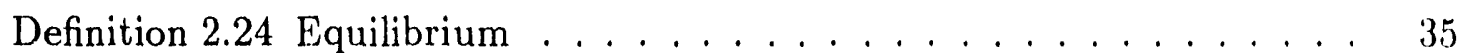

Theorem 2.25 Equilibrium Theorem . . . . . . . . . . . . . 36

Lemma $2.26 \ldots \ldots \ldots \ldots$

Lemma $2.27 \ldots \ldots \ldots$

Lemma $2.28 \ldots \ldots \ldots$

Lemma $2.29 \ldots \ldots \ldots$

Lemma $2.30 \ldots \ldots \ldots \ldots$ 


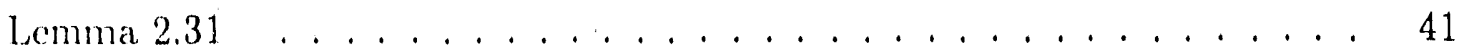

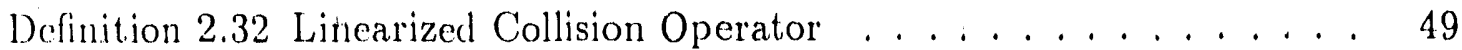

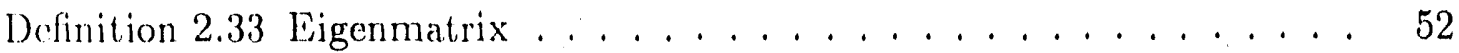

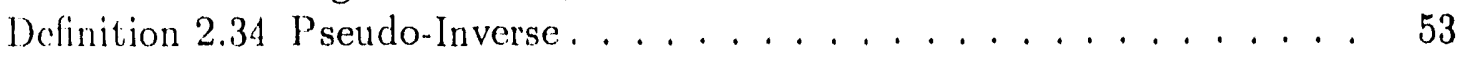

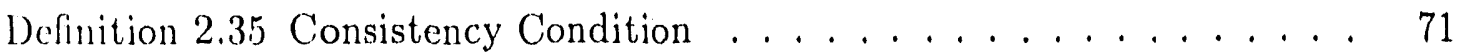

Definition 2.36 Hydrodynamical Equation . . . . . . . . . . 71

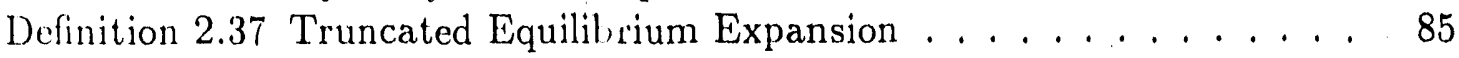

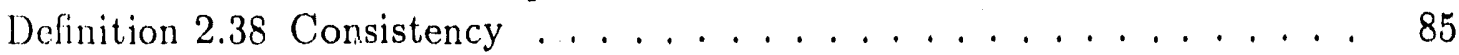

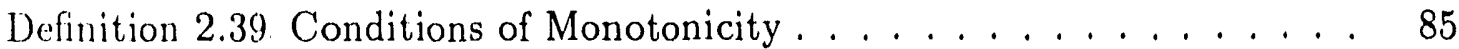

Definition 2.40 Domain of Monotonicity . . . . . . . . . . . 86

Definition $2.41 L\left[\mathbf{n}^{n}, \mathbf{h}^{n}\right] \ldots \ldots \ldots \ldots \ldots \ldots$

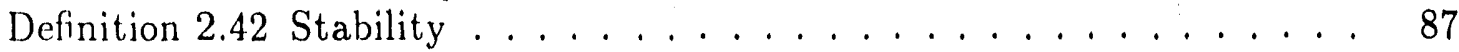

Deînition 2.43 Hydrodynamical Mode . . . . . . . . . . . . 87

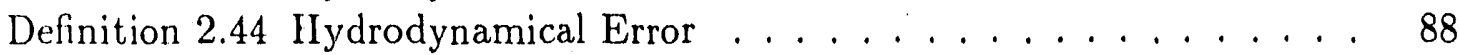

Lemma $2.45 \ldots \ldots \ldots \ldots$

Definition 2.46 Convergence $\ldots \ldots \ldots \ldots \ldots \ldots$

Theorem 2.47 Consistency . . . . . . . . . . . . . . . . . . . . . . . . . . . . . 89

Lemma 2.48 Consistency of $\mathrm{LB}_{1} \ldots \ldots \ldots \ldots$

Theorem 2.49 Discrete Maximum/Minimum Principle . . . . . . . . . 97

Lemma $2.50 \ldots \ldots$. . . . . . . . . . . . . . . . . . . 97

Lemma $2.51 \ldots \ldots \ldots$

Corollary $2.52 \ldots \ldots \ldots \ldots$

Lemma 2.53 Domain of Monot $n$ nicity for $\mathrm{LB}_{1} \ldots \ldots \ldots$. . . . . . . 99

Lemma 2.54 Discrete Maximum/Minimum Principle for $L_{1} B_{1} \ldots \ldots \ldots$

Lemma 2.55 Hydrodynamical Maximum/Minimum Principle for $\mathrm{LB}_{1} \ldots \ldots$

Lemma 2.56 Continuum Maximum/Minimum Principle for $\mathrm{LB}_{1} \ldots \ldots$. . . 111

Corollary $2.57 \ldots \ldots \ldots \ldots \ldots \ldots$

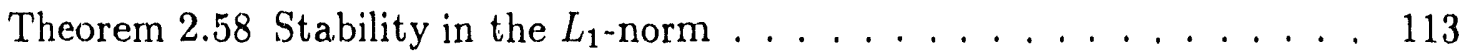

Lemma $2.59 \ldots \ldots \ldots \ldots \ldots \ldots$

Lemma 2.60 Stability of $\mathrm{LB}_{1}$ in the $L_{1}$-norm . . . . . . . . . . . . . 114

Theorem 2.61 Convergence . . . . . . . . . . . . . . . . . . . . 115

Theorem 2.62 Convergence of $\mathrm{LB}_{1} \ldots \ldots \ldots \ldots \ldots$

Lemma 3.1 Consistency of $\mathrm{LB}_{2} \ldots \ldots \ldots \ldots \ldots$

Lemma 3.2 Domain of Monotonicity for $\mathrm{LB}_{2} \ldots \ldots \ldots \ldots$

Lemma 3.3 Discrete Maximum/Minimum Principle for $\mathrm{LB}_{2} \ldots \ldots \ldots \ldots$

Lemma 3.4 Hydrodynamical Maximum/Minimum Principle for $\mathrm{LB}_{2} \ldots \ldots$

Lemma 3.5 Continuum Maximum/Minimum Principle for $\mathrm{LB}_{2} \ldots \ldots \ldots$

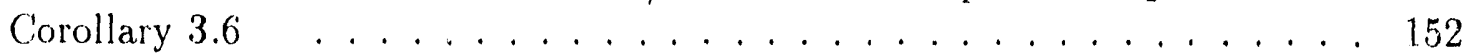

Lemma 3.7 Stability of $\mathrm{LB}_{2} \ldots \ldots \ldots \ldots \ldots \ldots$

Theorem 3.8 Convergence of $\mathrm{LB}_{2} \ldots \ldots \ldots \ldots \ldots$ 
Lemma 3.9 Consistency of LB Method for Burgers' Equation . . . . . . . 170

Lemma 3.10 Domain of Monotonicity of LB Method for Burgers' Equation 174

Lemma 3.11 Discrete Maximum/Minimum Principle of LB Method for Burgers' Equation . . . . . . . . . . . . . . . . . . . 175

Lemma 3.12 Maximum/Minimum Principle for the 1-D Viscous Burgers

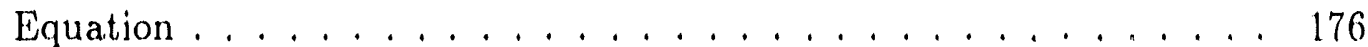

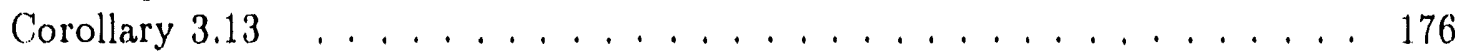

Lemma 3.14 Continuum Maximum/Minimum Principle of LB Method for Burgers' Equation . . . . . . . . . . . . . . . . . 176 Corollary $3.15 \ldots \ldots \ldots \ldots \ldots \ldots$

Lemma 3.16 Stability of LB Method for Burgers' Equation . . . . . . . . 178

Theorem 3.17 Convergence of LB Method for Burgers' Equation . . . . . . 178

Lemma C.1 Continuum Maximum Principle . . . . . . . . . . . . . . . 246

Lemma C.2 Discrete Maximum Principle . . . . . . . . . . . . . 247 


\section{List of Equations}

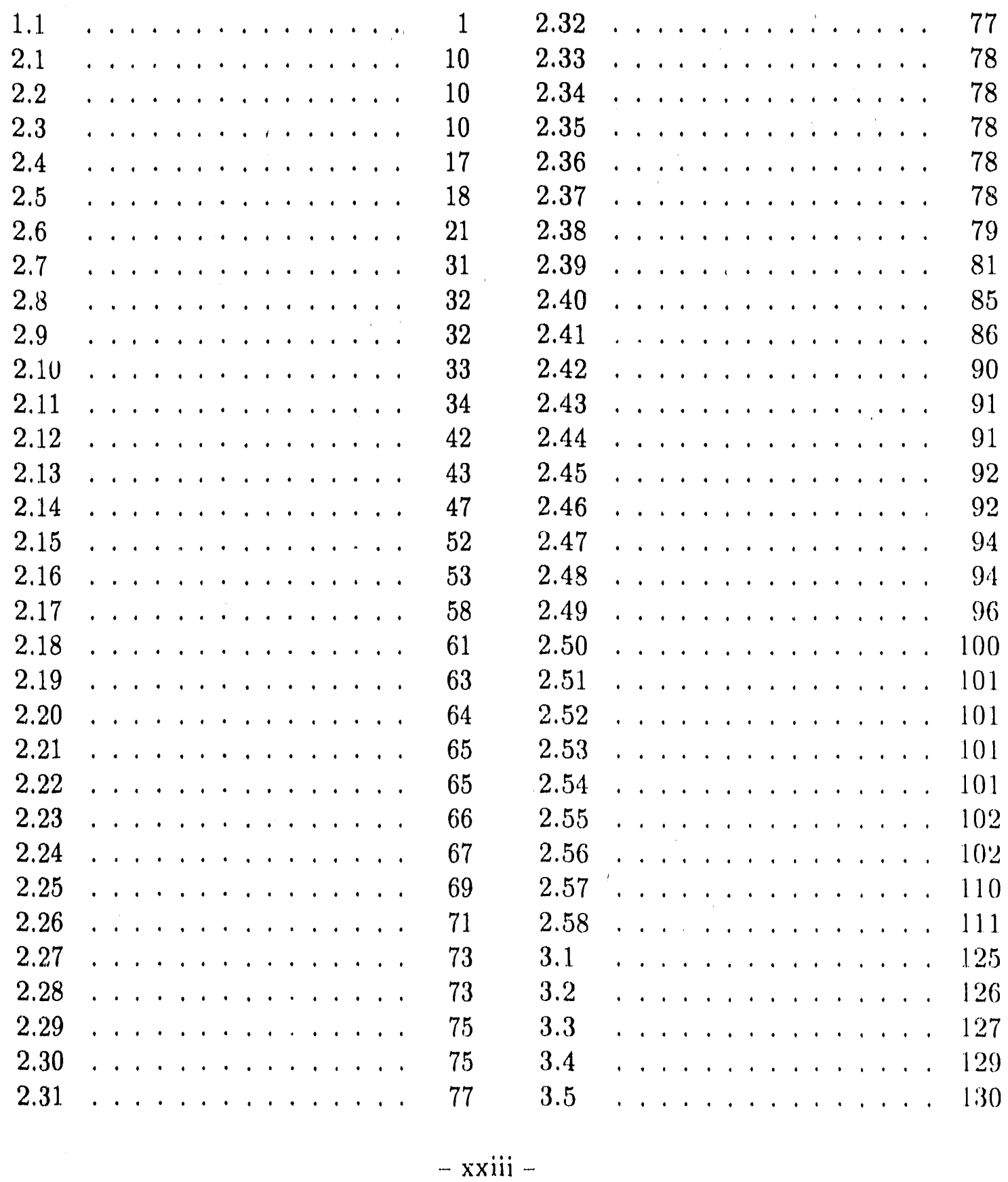




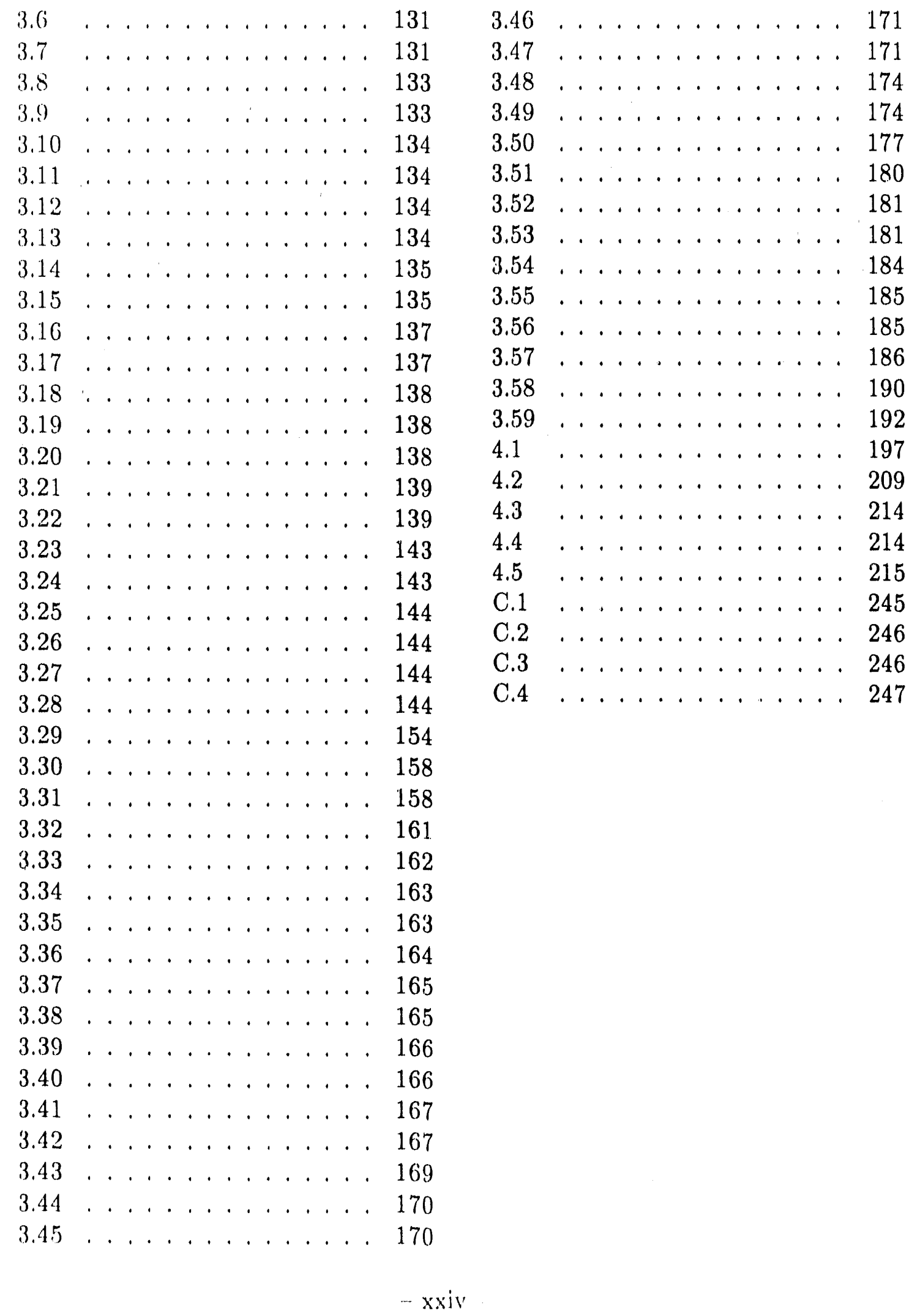




\section{Chapter 1}

\section{Introduction}

Consider the three-dimensional incompressible Navier-Stokes equations,

$$
\begin{aligned}
\frac{\partial}{\partial t} \vec{u}+(\vec{u} \cdot \nabla) \vec{u} & =-\frac{1}{\rho} \nabla P+\epsilon \Delta \vec{u} \\
\nabla \cdot \vec{u} & =0
\end{aligned}
$$

which connect the velocity $\vec{\imath}$ and pressure $P$, where $\rho$ is the constant density and $\epsilon$ is the kinematic viscosity. We would like to compute solutions to these equations with $0<\epsilon \leq 1$ efficiently and accurately for problems (to which Eq. 1.1 is only an approximation) with boundary and internal layers, shocks, turbulence, and complex domains. However, resolving accuracy of even simple problems with conventional methods far exceeds the current capability of computer resources [65]. The desired problems can easily require vasts amounts of memory and computation time, so much memory that the problems would not fit on current machines and so much time that it would surpass a lifetime. ${ }^{1}$ To make obtaining meaningful solutions to these problems more feasible we turn to the development of algorithms and supporting theory for parallel and vector computation. We encounter the same computational space-time

\footnotetext{
${ }^{1}$ A full Navier-Stokes calculation of turbulent flow over an aircraft with Reynolds numbers $5 \times 10^{7}$ to $3 \times 10^{8}$ (with 1988 algorithms) has been projected to take over $2 \times 10^{16}$ years; a large eddy simulation approximation to the Navier-Stokes equations, in which only large scale motions are computed from filtered Navier-Stokes equations and small scale motions are modeled, reduces the projection by about 5 orders of magnitude to around $2 \times 10^{11}$ years [62].
} 
problems for conventional, sequential methods transformed into parallel methods. Part of the difficulty in the development of parallel methods is that traditional training embraces sequential thinking processes. This dissertation investigates the numerical theory of some new conputational methods that are inherently parallel. The methods involve particles traversing the links of a lattice and interacting at the nodes. The particles arlvance in unison to neighboring nodes with unit speed according to a discrete clock. The system can be viewed as a discretization in time, space, and velocity of the Boltzmann equation, e.g., [37] (cf. $[15,59]$ ). However, the velocity space is coarsely discretized and does not refine in the limit as the unit spatial and temporal lengths ( $\Delta x$ and $\Delta t$ respectively) tend to zero. The fixed, coarse discretization of the velocity makes the approach amenable to a mathematical description (of the microdynamics) over a Boolean field, ${ }^{2}$ with an implementation using only logical operations, i.e., no floating point arithmetic. This point, in addition to the methods' vast implicit parallelism, has been argued in their favor. Although the microdynamics of these models is non-physical, several researchers have recovered macrodynamical descriptions that agree ${ }^{3}$ with those baser on physical microdynamics, e.g., $[37,38]$. Most models regard the macroscopic description of fluid dynamics, i.e., the NavierStokes equations. The models have been known as, among other terms, lattice gas automata, cellular automata, and cellular fluids. Obtaining the macrodynamical description involves averaging and an assumption regarding molecular chaos. Another related class of methods does not, however; they are lattice Boltzmann methods $[5,6,36,49,56]$. These methods originated from lattice gas methods, but differ in that particle distributions move along the links rather than particles themselves. In this

\footnotetext{
2The microdynamical description can also be made in terms of cellular automata, e.g., [72].

${ }^{3} \mathrm{~A}$ more precise term will be defined subsequently:
} 
way the molecular chaos assumption becomes an inherent part of the method, and the methods are floating point arithmetic calculations. Indeed, they are a form of finite difference method. Lattice gas and lattice Boltzmann methods (herein collectively termed "lattice methods") have been used in a number of computational arenas: incompressible fluid flow (Navier-Stokes) [37, 38, 46-48], magnetohydrodynamics [16, $17,58]$, the Poisson equation [18], mixed flows $[2,9,12-14,16]$, turbulence $[6,49]$, diffusion $[8,11,26,27,33]$, reaction-diffusion [28], advection-diffusion [7, 24, 53], and flow in porous media $[10,23] .{ }^{4}$ As the sur ject gains understanding, undoubtly, its scope and spectrum will increase.

However, lattice methods have lacked a formal numerical theory, which would include proving convergence through, e.g., consistency and stability arguments. With but one exception [7], numerical analysis of lattice methods only derives partial differential equations that describe the their macroscopic behavior; this is but the first part of a formal consistency argument. Consequently, many questions regarding their applicability, effectiveness, efficientness, competitiveness, and utility have remained open. Due mostly to limited knowledge about lattice methods, little progress toward answering the questions is being made. This dissertation addresses the issue by introducing a formal numerical theory for lattice methods and revealing that the methods are second-order, conservative, conditionally monotone finite difference methods for solving some partial differential equations. An attempt is made to formulate a foundation for rigorously treating convergence of the methods, including the consistency

\footnotetext{
${ }^{4}$ Note that the references listed provide examples of the application areas and are by no means meant to be exhaustive listings. The references, $[30,31,52,57]$, are collections of works and proceedings of conferences and workshops that encompass lattice methods (and related topics). Several of the articles contained therein have thorough reference lists, e.g., [37].
} 
and stability arguments. Another part of the dissertation investigates the applicability of domain decomposition techniques to the lattice methods, in an effo"t to better utilize computational resources, e.g., concentrate resources near highly active regions in the computational domain.

Chapter 2 introduces a numerical theory for lattice methods, the theory encompassing the microdynamical description, equilibria, the discrete Chapman-Enskog expansion in the derivation of associated partial differential equations, and convergence. Convergence is argued through " mal consistency and stability arguments similar to the ones made for nonlinear monotone schemes. Stability and convergence arguments are based on establishing maximum and minimum principles for both the lattice method its continuum approximation, i.e., its associated partial differential equation. The proof of the discrete maximum and minimum principles depends on, among other criteria, that a lattice method satisfies certain "conditions of monotonicity". These are the conditions under which a lattice method is a monotune finite difference method. As we will see, the conditions of monotonicity impose restrictions on the mean occupation numbers ${ }^{5}$ in a lattice method, i.e., that the mean occupation numbers must initially lie within the "domain of monotonicity". ${ }^{6}$ Convergence is proven on the basis that the occupation numbers are always within the domain of monotonicity. This is an important technical contribution of the dissertation, for it reveals that a lattice method may be unstable outside the domain of monotonicity.

\footnotetext{
${ }^{5}$ For now, the mean occupation numbers can be thought of as the average (or expected) numbers of particles per direction per node per time step.

${ }^{6}$ The domain of monotonicity, loosely speaking, is the largest domain in which the conditions of monotonicity are satisfied. And it will be shown that if the occupation numbers are initially within the domain of monotonicity, then as the system evolves they remain within the domain of monotonicity.
} 
An example is interleaved throughout this chapter to aid the presentation of the concepts and techniques. Chapter 3 applies the theory of Chapter 2 to three additional lattice methods. The first is a variation of the example lattice method in Chapter 2. The second method is for the one-dimensional Burgers equation. It was originally introduced as an advection-diffusion model in [7] and later studied as a lattice gas method in [53]. The third method is for two-dimensional advection-diffusion. Chapter 4 summarizes computational studies on all four of the lattice methods discussed in the dissertation. The results indicate agreement with the theoretical convergence rates for the methods. The chapter explores the behavior of the methods outside their domain of monotonicity (the proven realm of convergence), the results suggesting an extended realm of convergence in some cases. Largely based on the ideas in [60], Chapter 5 explores domain decomposition for lattice methods. The overall goal is to be able to concentrate computational resources on regions of high activity, such as steep gradients $[39,71]$, and boundary and internal layers [25, 32, 68]. The chapter introduces a possible strategy, one that might be used for explicit finite difference methods. Computational evidence supports the strategy and suggests that domain decomposition techniques can be applied to lattice methods in a manner to increase performance when only localized increased accuracy is desired. Chapter 6 is the conclusion. 


\section{Chapter 2}

\section{Theory}

This chapter introduces a numerical theory for lattice Boltzmann methods and the Lattice Boltzmann Approximation to lattice gas methods. The theory covers two techniques for determining equilibria of a lattice inethod, derivation of partial differential equations via the discrete Chapman-Enskog procedure, and convergence analysis. Convergence is obtained through formal consistency and stability arguments. Consistency is shown by a second apf ation of the discrete Chapman-Enskog procedure, while stability is shown by establishing discrete and continuum maximum and minimum principles for the lattice method, and showing that the method is a monotone finite difference method. An example is interleaved throughout the chapter.

Section 2.1 introduces notation and definitions of and for analysis of lattice gas and lattice Boltzmann methods. Section 2.2 provides two methods for determining equilibria of lattice methods. The first application (and introduction) of the discrete Chapman-Enskog analysis encompasses Section 2.3. And Section 2.4 introduces the convergence analysis. 


\subsection{Definitions and Notation}

This section defines terms and introduces notation for the subsequent discussions and analyses of lattice gas and lattice Boltzmann methods. We begin with introducing some vector notation and associated operations, e.g., exponentiation, differentials, gradients, and the Jacobian matrix. We then discuss norms, order of magnitude, and some set operations. Next, we discuss ensembles, ensemble averaging, and approximations to ensemble averaging. At this point we define lattice gas methods. And finally, we discuss the Lattice Boltzmann Approximation to lattice gas methods and use this to motivate the introduction of lattice Boltzmann methods.

\subsubsection{Basic Definitions}

Let us denote

$$
\begin{aligned}
\mathcal{B} & \equiv\{0,1\} \\
{[a, b] } & =\{x \mid a \leq x \leq b\} .
\end{aligned}
$$

Let $d$ be a positive integer indicating the number of directions in which particles may travel. Let

$$
\begin{aligned}
\mathbf{f}, \mathbf{g} & \in[0,1]^{d}(=\underbrace{[0,1] \times[0,1] \times \cdots \times[0,1]}_{d \text { times }}), \\
\alpha \in \mathcal{B}^{d} &
\end{aligned}
$$


and

$$
\mathbf{1}=\left[\begin{array}{c}
1 \\
1 \\
\vdots \\
1
\end{array}\right] \in[0,1]^{d} .
$$

Henceforth, boldface letters and numbers, e.g., $\mathbf{f}, \mathbf{g}, \mathbf{h}, \mathbf{n}, \mathbf{0}, \mathbf{1}$, denote column vectors of length $d$. Also, $\alpha$ and $\beta$ denote vectors in $\mathcal{B}^{d}$, although they are not in a boldface face. Let $f_{k}$ denote the $i$ th element of $\mathbf{f}$. Then in terms of $f_{k}$ we write $\mathbf{f}$ as

$$
\mathbf{f}=\left[f_{k}\right]_{k=0}^{k=d-1}
$$

Where clear the limits may be left off.

We define the following operations involving $\mathbf{f}, \mathrm{g} \in[0,1]^{d}, \alpha \in \mathcal{B}^{d}$, and $1 \in \mathcal{B}^{d}$ :

$$
\begin{array}{rlrl}
\mathbf{f}+\mathbf{g} & \equiv\left[f_{k}+g_{k}\right]_{k=0}^{d-1} & & \text { (componentwise vector sum) } \\
\mathbf{f} \star \mathbf{g} & \equiv\left[f_{k} g_{k}\right]_{k=0}^{d-i} & & \text { (componentwise vector product) } \\
\mathbf{f} \cdot \mathbf{g} & \equiv \sum_{i=0}^{d-1} f_{k} g_{k} & & (\text { dot product) }, \\
\overline{\mathbf{f}} & \equiv\left[1-f_{k}\right]_{k=0}^{d-1}=\mathbf{1}-\mathbf{f} & (\operatorname{read} \text { "not }(\mathbf{f}) "), \\
\bar{\alpha} & \equiv\left[1-\alpha_{k}\right]_{k=0}^{d-1}=1-\alpha & (\operatorname{read} " \operatorname{not}(\alpha) ") .
\end{array}
$$

The componentwise vector product $(\star)$ has the same precedence as scalar multiplication. Note that operations other than "not" $(\overline{\mathrm{f}})$ extend to vectors in $\tau^{d}$. Other operations like the logarithm are evaluated componentwise, e.g.,

$$
\log \mathbf{f}=\left[\log f_{k}\right]
$$


Definition 2.1 (Vector Exponentiation). Let $\mathbf{n} \in[0,1]^{d}$ and $\alpha \in \mathcal{B}^{d}$. Then define $\mathbf{n}^{\alpha} \in[0,1]$ by $\mathbf{n}^{\alpha} \equiv \prod_{i=0}^{d-1} n_{k}^{\alpha_{k}}$, in which $n_{k}^{0} \equiv 1$.

From the preceding definitions, we see that

$$
\begin{aligned}
\mathrm{n}^{\alpha} \overline{\mathbf{n}}^{\bar{\alpha}} & \equiv\left[\prod_{i=0}^{d-1} n_{k}^{\alpha_{k}}\right] \cdot\left[\prod_{i=0}^{d-1} \bar{n}_{k}^{\overline{\alpha_{k}}}\right] \\
& =\left[n_{0}^{\alpha_{0}} n_{1}^{\alpha_{1}} \cdots n_{d-1}^{\alpha_{d-1}}\right] \cdot\left[\left(1-n_{0}\right)^{\left(1-\alpha_{0}\right)}\left(1-n_{1}\right)^{\left(1-\alpha_{1}\right)} \cdots\left(1-n_{d-1}\right)^{\left(1-\alpha_{d-1}\right)}\right] \\
& =\prod_{i=0}^{d-1} n_{k}^{\alpha_{k}}\left(1-n_{k}\right)^{\left(1-\alpha_{k}\right)} \\
& =\prod_{i=0}^{d-1} n_{k}^{\alpha_{k}} \bar{n}_{k}^{\overline{\alpha_{k}}}
\end{aligned}
$$

a quantitity that appears, as we will see, in the collision operator (cf. Definition 2.18). And since $\alpha_{k} \in \mathcal{B}$, we find

$$
n_{\dot{k}}^{\alpha_{k}}{\overline{n_{k}}}^{\overline{\alpha_{k}}}=n_{k}^{\alpha_{k}}\left(1-n_{k}\right)^{\left(1-\alpha_{k}\right)}= \begin{cases}n_{k}, & \alpha_{k}=1 \\ \overline{n_{k}}, & \alpha_{k}=0\end{cases}
$$

Note also that

$$
\alpha \cdot \log \mathbf{n}=\sum_{i=0}^{d-1} \alpha_{k} \log n_{k}=\log \prod_{i=0}^{d-1} n_{k}{ }^{\alpha_{k}}=\log \mathbf{n}^{\alpha}
$$

\section{Differentials}

Let $\mathcal{C}(\mathbf{n})$ denote a vector-valued function $\mathcal{C}:[0,1]^{d} \rightarrow[0,1]^{d}$. Denote the $k$ th function $\mathcal{C}_{k}(\mathrm{n})$. Let $\mathrm{h}^{(0)}, \mathrm{h}^{(1)}, \ldots \mathrm{h}^{(j-1)} \in[0,1]^{d}$. Then define the $j$ th differential of $\mathcal{C}(\mathbf{n})$ as

$$
\begin{aligned}
& \frac{\partial^{j}}{\partial \mathbf{n}^{j}} \mathcal{C}(\mathbf{n}) \cdot \mathbf{h}^{(0)} \mathbf{h}^{(1)} \ldots \mathbf{h}^{(j-1)} \\
& \equiv\left[\sum_{k_{0}=0}^{d-1} \sum_{k_{1}=0}^{d-1} \cdots \sum_{k_{j-1}=0}^{d-1}\left(\frac{\partial^{j} \mathcal{C}_{-k}(\mathbf{n})}{\partial n_{k_{0}} \partial n_{k_{1}} \cdots \partial n_{k_{-1}}}\right) h_{k_{0}}^{(0)} h_{k_{:}}^{(1)} \cdots h_{k_{j-1}}^{(j-1)}\right]_{k=0}^{k=d-1} \\
& \quad \in[0,1]^{d} .
\end{aligned}
$$


The $j$ th differential of $\mathcal{C}(\mathbf{n})$ evaluated for $\mathbf{n}=\mathbf{n}^{(0)}$ is written

$$
\begin{aligned}
& \frac{\partial^{j}}{\partial \mathbf{n}^{j}} \mathcal{C}\left(\mathbf{n}^{(0)}\right) \cdot \mathbf{h}^{(0)} \mathbf{h}^{(1)} \cdots \mathbf{h}^{(j-1)} \\
& \quad=\left[\left.\sum_{k_{0}=0}^{d-1} \sum_{k_{1}=0}^{d-1} \ldots \sum_{k_{j-1}=0}^{d-1}\left(\frac{\partial^{j} \mathcal{C}_{k}(\mathbf{n})}{\partial n_{k_{0}} \partial n_{k_{1}} \cdots \partial n_{k_{j-1}}}\right)\right|_{\mathbf{n}=\mathbf{n}^{(0)}} h_{k_{0}}^{(0)} h_{k_{1}}^{(1)} \cdots h_{k_{j-1}}^{(j-1)}\right]_{k=0}^{k=d-1} \\
& \quad \in[0,1]^{d} .
\end{aligned}
$$

The Jacobian matrix of $\mathcal{C}(\mathbf{n})$ is written $\frac{\partial}{\partial \mathbf{n}} \mathcal{C}(\mathbf{n})=\left[c_{k, l}\right]_{k, l=0}^{d-1}$, where $c_{k, l}=\frac{\partial}{\partial n_{l}} \mathcal{C}_{k}(\mathbf{n})$.

We may use the shorthand notation $\mathcal{D}^{j} \equiv \frac{\partial^{j}}{\partial \mathbf{n}^{j}} \mathcal{C}(\mathbf{n})$.

\section{Norms}

In the convergence analysis, we use norms over discrete and continuum quantities. We will use the same notation for each with the context determining the appropriate definition.

The $\ell_{p}$-norm for a function $u(\vec{x} ; t)$ with $\vec{x} \in \Omega=[0, L]^{D}$ is defined by

$$
\|u(\vec{x} ; t)\|_{\ell_{p}} \equiv\left[\int_{\Omega}|u(\vec{x} ; t)|^{p} d \vec{x}\right]^{1 / p}, \text { for } p \geq 1
$$

if the integral exists and is finite, and

$$
\|u(t)\|_{\ell_{\infty}} \equiv \lim _{p \rightarrow \infty}\|u(\vec{x} ; t)\|_{\ell_{p}}
$$

if the limit exists and is finite.

Let $U_{\vec{i}}^{n}$ be an approximation to $u(\vec{\imath} \Delta x ; n \Delta t)$ with $\vec{x} \in \Omega=[0, L]^{D}$. Then the $\ell_{p}$-norm of $U$ is defined by

$$
\left\|U^{n}\right\|_{\ell_{p}} \equiv\left(\left(\frac{\Delta x}{L}\right)^{D} \sum_{i}\left|U_{i}^{n}\right|^{p}\right)^{1 / p} .
$$


Note that

$$
\left\|U^{n}\right\|_{\ell_{\infty}} \equiv \lim _{p \rightarrow \infty}\left\|U^{n}\right\|_{\ell_{p}}=\sup _{i}\left|U_{i}^{n}\right|
$$

\section{Order of Magnitude'}

DEFinition 2.2 (ORDER OF MAGNitude). We say function $g(\epsilon$ ) is on the order of the magnitude of $f(n)$, which is denoted $g(\epsilon)=\mathcal{O}[f(n)]$, if there exists a constant c such that $\lim _{\epsilon \rightarrow 0} f(\epsilon) / g(\epsilon)=c$.

\section{Sets}

The following definitions are from [55, p. 68 and p. 258], modified for lattice methods.

Definition 2.3 (CONNECTEDNESS). We say a set $A \subset \mathcal{R}^{d}$ is connected if there do not exist two non-empty, open sets, $B$ and $C$, such that $A \subset B \cup C, A \cap B \neq \emptyset$, $A \cap C \neq \emptyset$, and $A \cap B \cap C=\emptyset$.

Definition 2.4 (SET VolumE). If $\mathcal{E} \subset[0,1]^{d}$, define the characteristic function $1_{\mathcal{E}}(\mathbf{n})$ of $\mathcal{E}$ by

$$
1_{\mathcal{E}}:[0,1]^{d} \rightarrow \mathcal{R}, \quad 1_{\mathcal{E}}(\mathbf{n})= \begin{cases}1, & \mathrm{n} \in \mathcal{E} \\ 0, & \mathrm{n} \notin \mathcal{E}\end{cases}
$$

We say that $\mathcal{E}$ has volume if ${ }^{1} \mathcal{E}$ is integrable, and the volume of $\mathcal{E}$ is the number

$$
\int_{\mathcal{E}}{ }^{1} \mathcal{E}^{(\mathbf{n}) d \mathbf{n}=v(\mathcal{E})}
$$

(Note that it makes sense to talk about the integrability of ${ }^{1} \mathcal{E}$ since $\mathcal{E}$ is a bounded set.) 


\section{Ensembles}

An ensemble is a possibly infinite collection of events or values. The ensemble average is the expected or average value of the events or values. Let $\hat{Q}$ denote a particular event in an ensemble. Then its ensemble average is written $\langle\hat{Q}\rangle$. Henceforth, when discussing ensembles and ensemble averages, a quantity without a hat (" + ") is taken to be the ensemble average, e.g., $Q=\langle\hat{Q}\rangle$.

It is oftei convenient to discuss the correlation between two collections of events. One mathematical tool for doing so is the covariance, which is defined as follows:

Definition 2.5 (Covariance). Let $A$ and $B$ be two collections of events. Let $\hat{a}$ be a random event in collection $A$; let $\hat{b}$ be a random event in collection $B$. Then from probability theory, e.g., [50], the covariance between $A$ and $B, \operatorname{Cov}(A, B)$, is defined by

$$
\operatorname{Cov}(A, B) \equiv\langle\hat{a} \hat{b}\rangle-\langle\hat{a}\rangle\langle\hat{b}\rangle
$$

The covariance measures the dependence between two collections; ${ }^{1}$ the covariance between two collections is zero if they are independent, i.e., statistically uncorrelated. In the abscence of having labels for collections, we may write

$$
\operatorname{Cov}(\hat{a}, \hat{b})=\langle\hat{a} \hat{b}\rangle-\langle\hat{a}\rangle\langle\hat{b}\rangle
$$

with the meaning understood.

For spatially and temporally dependent data, there are a variety of methods for approximating the ensemble average with as few as one instance (event) of an ensemble, the simplest being to average over the few instances. Spatial and temporal averaging may be used when certain assumptions regarding correlations can be made,

\footnotetext{
ite that the concept of covariance can be extended to measure the correlation among multiple colleweris.
} 
e.g., crgodicity. (For systems of particles such an assumption may regard molecular (haos" when using spatial averaging.) The validity of temporal averaging depends on how slowly and locally the data evolves in time. Combinations of all three methods may be used to approximate the ensemble average for a possibly infinite ensemble. Lattice gas calculations have, in general, used the spatial averaging approach (applied to one instance).

\subsubsection{Lattice Gas Methods}

This dissertation focuses on one-speed models, although the concepts can easily be extended to multi-speed models, including models with rest particles, i.e., particles with no velocity. In the one-speed models, particles move about in unison according to the ticks of a discrete clock along the links of a regular lattice with unit speed and interacting at the nodes of the lattice; a set of collision rules determines the nature of the interactions. The interactions change the velocity of the particles, which are indistinguishable, and the outcomes are deterministic or nondeterministic, depending the collision rules. Lattice gas methods are inherently designed with the Fermi Exclusion Principle.

Definition 2.6 (Fermi Exclusion Principle). The Fermi Exclusion Principle (for lattice gas methods) allows at any given time, at most one particle per direction per node. ${ }^{3}$

Adhering to this principle ensures either absence or presence of a particle moving in

\footnotetext{
${ }^{2}$ See, e.g., $[64$, p. 523] for a discussion of the molecular chaos assumption.

${ }^{3}$ Note that one can adhere to this rule and obtain multiple particles per direction by duplicating directions where increased numbers of particles are desired to be allowed. Each "same" direction would have different direction labels, though they would denote (through interpretation) identical physical directions.
} 
a particular direction at a node. It is in this way that a finite number of bita may be: used to describe the state at a node--one bit for each direction. Supposing that cach node has $d$ links, a node state may be described as an eloment, of a Boolean field, $\mathcal{B}^{b}$.

Toward building notation for quantifying a latice gas method's behavior, the lattice gas methods discussed have the following characteristics: a doubly periodic (toroidal) regular lattice suspended in $p$-dimensional space, $\Omega=[0, L]^{p}$, with $d$ links per node, each link having length $\Delta x=L \delta$; time advances in units of $\Delta t=T^{\prime} \delta^{2}$, where $L$ and $T$ are the spatial and temporal scale lengths, respectively. We adopt the following from $[37, \mathrm{pp}, 657-9]$ :

Definition 2.7 (LATTTICE), Let Bravais lattice, $\mathfrak{L} \subset \mathcal{R}^{p}$, be such that there exists a set of $d$ velocity vectors, $\vec{e}_{k}, k \in\{0,1, \ldots, d-1\}$, having equal modulus (the modulus, $c$, is called the particle speed). Regarding the velocity vectors, we adopt the notation

$$
\vec{e}_{k}=\left[e_{k, i}\right]_{i=0}^{p-1} .
$$

We may refer to the velocity vectors as the unit velocity vectors. Of $\mathfrak{L}$ and the velocity vectors, we require:

1. $\left\{\vec{\imath}+\vec{e}_{k} \mid k \in\{0,1, \ldots, d-1\}\right\} \subset \mathfrak{L}$ is the set of nearest neighbors of lattice node $\vec{\imath} \in \mathfrak{L}$,

2. for any two nodes $\vec{\imath}, \vec{\jmath} \in \mathfrak{L}$, there exists a finite sequence of velocity vectors,

$$
\vec{e}_{k_{0}}, \vec{e}_{k_{1}}, \ldots, \vec{e}_{k_{P}}
$$

such that $\vec{\imath}=\vec{\jmath}+\sum_{p=0}^{P} \vec{e}_{k_{p}}$.

3. For any pair of velocity vectors, $\left(\vec{e}_{k}, \vec{e}_{l}\right)$, there exists an element, which maps 
from $\vec{e}_{k}$ to $\vec{e}_{1}$, in the crystallographic group $\mathfrak{G}$ of isometries globally preserving the set of velocity vectors.

4. Let $\mathfrak{G}_{k}$ denote the subgroup of $\mathfrak{B}$ that leaves velocity vector $\vec{e}_{k}$ invariant. Let $\mathfrak{H}_{k}$. be the orthogonal hyperplane of $\vec{e}_{k}$. Then there is no non-vanishing vector in $\mathfrak{H}_{k}$ that is invariant under all the elements of $\mathfrak{G}_{k}$, and the only linear transformations within the space $\mathfrak{H}_{k}$ that commute with all the elements of $\mathfrak{G}_{k}$ are proportional to the identity in $\mathcal{R}^{p}$.

Definition 2.8 (OCCUPATION NUMBER). The occupation number denotes the number of particles ( 0 or 1) with a particular velocity at a node. We denote this $\left(\hat{n}_{k}\right)_{\mathfrak{r}}^{n} \in \mathcal{B}$ for the number of particles in direction $k$ at lattice node $\vec{\imath} \in \mathfrak{L}$ and time step $n$. For a particular node, e.g., $(\vec{\imath} ; n)$, we denote the vector of occupation numbers

$$
\hat{\mathbf{n}}_{\hat{r}}^{n} \equiv\left[\left(\hat{n}_{k}\right)_{\hat{r}}^{n}\right]_{k=0}^{k=d-1}
$$

where $d$ is the number of directions on the lattice.

We may sometimes use the alternative notation $\hat{n}_{k}\left(\vec{l}_{;}^{\prime} n\right)=\left(\hat{n}_{k}\right)_{r}^{n}$. Note that $n$ used as a superscript denotes a time step.

At this point we introduce an example that will be interleaved throughtout the text. We will refer to this example as new material is introduced. The example lattice method, we will find, is a model for diffusion via a two-dimensional nonlinear diffusion equation.

\footnotetext{
${ }^{4}$ Examples are typeset in Sans Serif fonts at a slightly smaller pointsize than the surrounding text. Their margins are slightly reduced, also.
} 


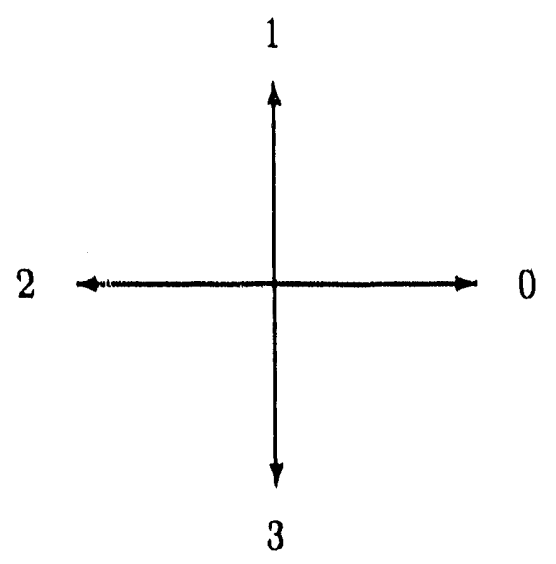

Figure 2.1: $L B_{1}$ direction labels.

Example ( $L B_{1}$ : Its Lattice and Velocity Vectors).

Consider a two-dimensional deterministic lattice gas method, called " $L B_{1}$ ", with a square lattice. Its cells are also squares. Let each edge have unit length $\Delta x$ with time advancing in units of $\Delta t$. There are four directions in which particles may travel to nearest neighbors, so $n=4$. Let us label these directions $0,1,2$, and 3 in a counterclockwise fashion with 0 being to the right. Pictorially, this is shown in Figure 2.1. We see $\left(\hat{n}_{0}\right)_{r}^{n}$ is the number of particles (either 0 or 1 ) moving in direction 0 at position $\left(\vec{\imath}_{i}, \imath\right)$. Also, $\hat{\mathbf{n}}_{\mathfrak{q}}^{n} \in \mathcal{B}^{4}$ is a vector of occupation numbers. Here, $\vec{i}=(i, j)$ is the vector of indices over the two-dimensional lattice, where $i$ and $j$ index horizontal and vertical positions on the lattice respectively. Unless stated otherwise we assume that $\hat{\mathbf{n}}$ is evaluated at $(\vec{i}, n)$. Note that the unit direction vectors are

$$
\begin{aligned}
& \vec{e}_{0}=(1,0), \\
& \vec{e}_{1}=(0,1), \\
& \vec{e}_{2}=(-1,0), \\
& \vec{e}_{3}=(0,-1),
\end{aligned}
$$


Let the coordinate axes be given by $t=\left(x_{0}, x_{1}\right)=(x, y)$, where the $x$-axis is in the direction of $\vec{e}_{0}$.

Let $u s$ verify 5 that the lattice and velocity vectors for $L B_{1}$ satifies the requirements of Definition 2.7. Note that permutations and reversals of the $x$ - and $y$. coordinates generate the isometry group $\mathbb{B}$ of the set of velocities, $\left\{\vec{e}_{0}, \vec{e}_{1}, \vec{e}_{2}, \vec{e}_{3}\right\}$. Any $\vec{e}_{k}$ can be mapped to $\ddot{e}_{l}$ by the above isometries; for example, $\vec{e}_{0}$ maps to $\vec{e}_{3}$ by permuting the $x$-and $y$-coordinates of $\vec{e}_{0}$, and then reversing the sign of the $y$-coordinate in the result. Let $g_{k}$ denote the subgroup of $\mathfrak{B}$ that leaves $\vec{e}_{k}$ invariant. We see that in each case, the subgroup consists of the identity and reversal of the $(k+1(\bmod 2))$-coordinate. In addition, the orthogonal hyperplane to $\vec{e}_{k}$ is $\left.\mathfrak{H}_{k}=\operatorname{span}\left(\vec{e}_{(k+1}(\bmod 2)\right)\right)$. There is no non-zero vector in $\mathfrak{H}_{k}$ that is invariant under the subgroup $\mathbf{g}_{k,}$ and the only linear transformations within $\mathfrak{H}_{k}$ and commuting with all elements of $\mathrm{g}_{k}$ are proportional to the identity operator.

\section{Microdynamical Equation}

'The behavior of a lattice gas is governed directly by its rules: rules for advecting particles to new locations and rules for determining new directions of the particles. 'These are characterized in the microdynamical evolution equation for a lattice gas method:

$$
\mathcal{A} \hat{\mathbf{n}}_{\mathfrak{i}}^{n}=\hat{\mathbf{n}}_{\mathfrak{i}}^{n}+\mathcal{C}\left(\hat{\mathbf{n}}_{\hat{i}}^{n}\right)
$$

where $\mathcal{A}$ and $\mathcal{C}$ are the advection and collision operators, respectively.

The microdynamical equation, Eq. 2.5, says that the new occupation numbers (on the left $)$ at the new locations, $\left(\vec{\imath}+\vec{e}_{k} ; n+1\right)$, are the same as the occupations numbers

\footnotetext{
${ }^{5}$ Note that $L B_{1}$ shares the same lattice and set of velocity vectors as the HPP model [48] for some two-dimensional Navier-Stokes-like equations. Verification of the requirements for the lattice and set of velocity vectors of the IIP' model is given in [37, p. (39)]). We repent the verification here for completerness.
} 
at the current location, $(\vec{i} ; n)$, plus some correction for particles changing directions. The term, $(\hat{\mathbf{n}})_{r}^{n}$, is the vector of occupation numbers at $(\vec{l} ; n)$. The collision operator, $\mathcal{C}$, changes the direction of particles at $(\vec{i} ; n)$ before the advection. Then the sum,

$$
\hat{\mathbf{n}}(\vec{\imath} ; n)+\mathcal{C}(\hat{\mathbf{n}}(\vec{\imath} ; n)),
$$

describes the new occupation numbers that are to be advected.

DEFINITION 2.9 (ADVECTION OPERATOR), Let a lattice, $\mathfrak{L}$, and its associated unit velocity vectors, $\left\{\vec{e}_{0}, \vec{e}_{1}, \ldots, \vec{e}_{d-1}\right\}$, be given. Then then advection operator, $\mathcal{A}$, is defined by

$$
\mathcal{A} \hat{\mathbf{n}}_{\tilde{\gamma}}^{n}=\left[\begin{array}{c}
\left(\hat{n}_{0}\right)_{i+\tilde{e}_{0}}^{n+1} \\
\left(\hat{n}_{1}\right)_{\tilde{r}+\tilde{e}_{1}}^{n+1} \\
\vdots \\
\left(\hat{n}_{d-1}\right)_{i+\tilde{e}_{d-1}}^{n+1}
\end{array}\right]=\left[\left(\hat{n}_{k}\right)_{\hat{\tau}+\tilde{e}_{k}}^{n+1}\right]_{k=0}^{d-1} .
$$

The advection operator, $\mathcal{A}$, is used to describe mathematically the movement of particles to their new locations.

Note that the microdynamical equation may be written in a more familiar difference equation format, i.e., without the advection operator,

$$
\hat{\mathbf{n}}_{\mathfrak{r}}^{n+1}=\left[H_{k}\left(\hat{\mathbf{n}}_{\tilde{\mathfrak{r}}-\vec{e}_{k}}^{n}\right)\right]_{k=0}^{d-1},
$$

where

$$
H_{k}\left(\hat{\mathbf{n}}_{\mathfrak{r}-\tilde{e}_{k}}^{n}\right)=\left(\hat{n}_{k}\right)_{\Gamma_{-} \vec{e}_{k}}^{n}+\mathcal{C}\left(\hat{\mathbf{n}}_{\mathfrak{\mathbf { r }}-\vec{e}_{k}}^{n}\right) .
$$

\section{State Transition Function and Collision Rules}

First of all, note that each lattice node has a state that describes its configuration. We have denoted this state with the occupation numbers, $\hat{\mathbf{n}}_{\mathfrak{r}}^{n}$. Because $\hat{\mathbf{n}}_{\mathfrak{r}}^{n} \in \mathcal{B}^{d}$, there 
are exactly $2^{d}$ possible configurations or states that a particular lattice location can have. Given that a location is in state $\alpha \in \mathcal{B}^{d}$, say, e.g., $\alpha=\hat{\mathrm{n}}_{\mathrm{r}}^{n}$, then there are $2^{d}$ possible new states, $\beta \in \mathcal{B}^{d}$. Depending on the lattice gas method, there will be a certain probabilility that a location in state $\alpha$ will change to state $\beta$ before the advection occurs. This is determined by the collision operator. We can define a state transition function that maps these probabilities for the finite number of $\alpha$ and $\beta$ combinations.

Definition 2.10 (STAte Transition Function). Let the set of possible states at a lattice node in a lattice gas method be $\mathcal{B}^{d}$. Let $\mathcal{S}: \mathcal{B}^{d} \times \mathcal{B}^{d} \rightarrow[0,1]$ be defined by

$$
\mathcal{S}(\alpha \rightarrow \beta)=\mathrm{P}\{\alpha \rightarrow \beta\}
$$

where

$$
\sum_{\beta \in \mathcal{B}^{d}} \mathcal{S}(\alpha \rightarrow \beta)=1 \quad \forall \alpha \in \mathcal{B}^{d}
$$

i.e., $\mathcal{S}$ obeys conservation of probability. Then $\mathcal{S}(\alpha \rightarrow \beta)$ is the probability of a transition from state $\alpha$ to state $\beta$, independent of location. $\mathcal{S}(\alpha \rightarrow \beta)$ is called the state transition function of a lattice gas method.

The state transition function determines the probability of a lattice node changing state prior to advection. Let us put this in terms of the lattice particles. First, let $\alpha$ be the state at a particular lattice node. Then it describes the particles and their respective directions at that node. Let $\beta$ be a new state at the same lattice node prior to advection, so that $\beta$ describes the particles and their new directions. Note that $\alpha$ and $\beta$ may, of course, be different. Given that a node is in state $\alpha$, the probability that it will change to state $\beta$ prior to advection, is $\mathcal{S}(\alpha \rightarrow \beta)$. If the number of particles in $\alpha$ and $\beta$ are the same, i.e., mass is conserved, then the state transition 
function describes the proces's of particles obtaining new directions.

We assume that the state transition function may be expressed in a finite sum in powers of $\delta, \forall \delta \in[0,1]$, i.e.,

$$
\mathcal{S}(\alpha \rightarrow \beta)=\sum_{j=0}^{J} \delta^{j} \mathcal{S}^{(j)}(\alpha \rightarrow \beta)
$$

for some $J \geq 0, \forall \alpha, \beta \in \mathcal{B}^{d}$. Such an expansion exists for all the methods studied herein (as well as all other one-speed lattice method methods like FHP [38] and FCHC [37]); it is the case that $J=0$ (for the diffusion models) and $J=1$ (for the advection-diffusion mod,els).

We adopt the following from [37, p. 660], which also discusses further invarianceand isotropy-related properties:

DEFINITION 2.11 (LATTICE ISOMETRY IriVARIANCE). Let $\mathcal{S}$ have the expansion in Eq. 2.6. A state transition function, $\mathcal{S}$, is said to have lattice isometry invariance if $\mathcal{S}^{(0)}$ is invariant under all isometries preserving the set of velocity vectors, i.e.,

$$
\mathcal{S}^{(0)}(\mathbf{g}(\alpha) \rightarrow \mathbf{g}(\beta))=\mathcal{S}^{(0)}(\alpha \rightarrow \beta), \quad \forall \mathbf{g} \in \mathfrak{G}, \quad \forall \alpha, \beta \in \mathcal{B}^{d}
$$

DEFINITION 2.12 (COLlision RULES). The set of collision rules of a lattice gas method is the tabulation of the mapping of its state transition function.

\section{Example ( $L B_{1}$ : Collision Rules).}

The collision rules for $\mathrm{LB}_{1}$ are exhibited in Figure 2.2 and listed in Table 2.1.

Definition 2.13 (Conserved Quantity). Let $\mathbf{a} \in[0,1]^{d}$. Then a is said to be $a$ conserved quantity if

$$
\sum_{k=0}^{d-1}\left(\beta_{k}-\alpha_{k}\right) \mathcal{S}(\alpha \rightarrow \beta) a_{k}=0 \quad \forall \alpha, \beta \in \mathcal{B}^{d} .
$$


Table 2.1: $\mathrm{LB}_{1}$ Collision Rules.

\begin{tabular}{||c||cccc|cccc|c||}
\hline \hline \multicolumn{1}{||c||}{ Rule } & \multicolumn{5}{|c|}{$\alpha$} & \multicolumn{5}{|c||}{$\beta$} & $\mathcal{S}(\alpha \rightarrow \beta)$ \\
\cline { 2 - 9 } & $\alpha_{0}$ & $\alpha_{1}$ & $\alpha_{2}$ & $\alpha_{3}$ & $\beta_{0}$ & $\beta_{1}$ & $\beta_{2}$ & $\beta_{3}$ & \\
\hline \hline 0 & 0 & 0 & 0 & 0 & 0 & 0 & 0 & 0 & 1 \\
1 & 0 & 0 & 0 & 1 & 0 & 0 & 0 & 1 & 1 \\
2 & 0 & 0 & 1 & 0 & 0 & 0 & 1 & 0 & 1 \\
3 & 0 & 0 & 1 & 1 & 1 & 1 & 0 & 0 & 1 \\
4 & 0 & 1 & 0 & 0 & 0 & 1 & 0 & 0 & 1 \\
5 & 0 & 1 & 0 & 1 & 1 & 0 & 1 & 0 & 1 \\
6 & 0 & 1 & 1 & 0 & 1 & 0 & 0 & 1 & 1 \\
7 & 0 & 1 & 1 & 1 & 0 & 1 & 1 & 1 & 1 \\
8 & 1 & 0 & 0 & 0 & 1 & 0 & 0 & 0 & 1 \\
9 & 1 & 0 & 0 & 1 & 0 & 1 & 1 & 0 & 1 \\
10 & 1 & 0 & 1 & 0 & 0 & 1 & 0 & 1 & 1 \\
11 & 1 & 0 & 1 & 1 & 1 & 0 & 1 & 1 & 1 \\
12 & 1 & 1 & 0 & 0 & 0 & 0 & 1 & 1 & 1 \\
13 & 1 & 1 & 0 & 1 & 1 & 1 & 0 & 1 & 1 \\
14 & 1 & 1 & 1 & 0 & 1 & 1 & 1 & 0 & 1 \\
15 & 1 & 1 & 1 & 1 & 1 & 1 & 1 & 1 & 1 \\
\hline \hline
\end{tabular}




\section{STATE PRE-COLLISION $\quad$ POST-COLLISION}

No Particles

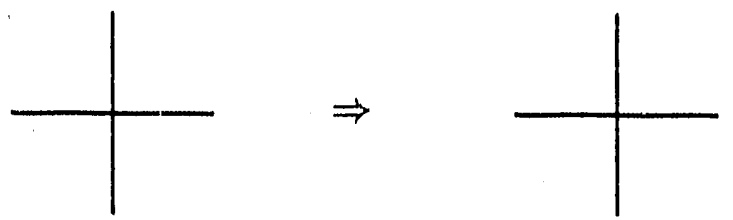

One Particle
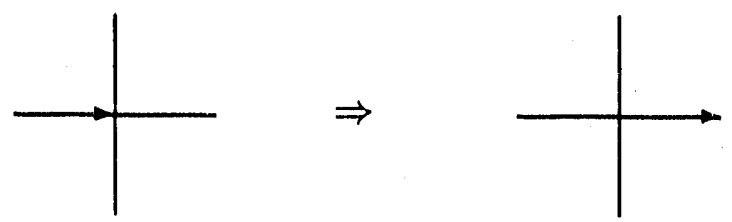

Two Orthogonal Particles
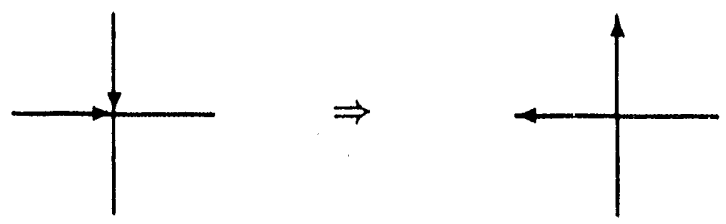

Two Head-on Particles
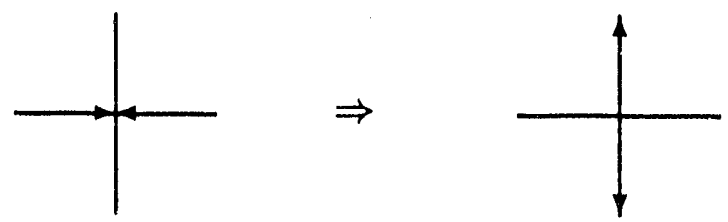

Three Particles
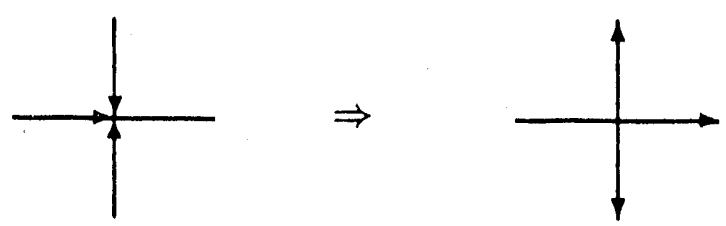

Four Particles
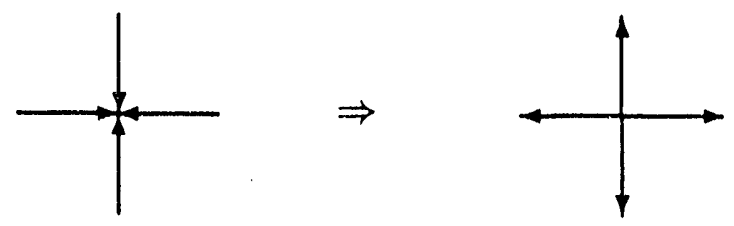

Figure 2.2: $\mathrm{LB}$, collision rules. The only states in which particles change direction are those with exactly two particles present. In both the orthogonal and head-on cases, particles change direction to the unoccupied directions. 
Conservation of mass and momentum are related to

$$
a_{k} \in \operatorname{span}(1) \quad \text { and } \quad a_{k} \in \operatorname{span}\left(e_{k, 0}, e_{k, 1}, \ldots, e_{k, p-1}\right) \text {, }
$$

respectively. We assume herein that these are the only types of conservation laws that a lattice method may have.

Definition 2.14 (Mass-Conserving). A lattice method is said to be massconserving if

$$
\sum_{k=0}^{d-1}\left(\beta_{k}-\alpha_{k}\right) \mathcal{S}(\alpha \rightarrow \beta)=0 \quad \forall \alpha, \beta \in \mathcal{B}^{d}
$$

The important concepts of detailed balance and semi-detailed balance are defined as follows: ${ }^{6}$

Definition 2.15 (Detailed BALANCE). Let $\mathcal{S}(\alpha \rightarrow \beta)$ be the state transition function of a lattice method method. Then if $\mathcal{S}(\alpha \rightarrow \beta)=\mathcal{E}(\beta \rightarrow \alpha) \forall \alpha, \beta \in \mathcal{B}^{d}$, we say that the lattice method satisfies detailed balance.

Example ( $L B_{1}$ : Detailed Balance).

To show that $L B_{1}$ satisfies detailed balance, we must verify that each collision rule satisfies detailed balance. As an example let us verify that rule 3 satisfies detailed balance. In rule 3 ,

$$
\mathcal{S}\left(\left[\begin{array}{l}
0 \\
0 \\
1 \\
1
\end{array}\right] \rightarrow\left[\begin{array}{l}
1 \\
1 \\
0 \\
0
\end{array}\right]\right)=1
$$

\footnotetext{
${ }^{6}$ They play important roles in (1) determining equilibrium solutions of a lattice method, and (2) guaranteeing that the linearized collision operator is nonpositive definite.
} 
and checking the table, we find (see rule 12)

$$
\mathcal{S}\left(\left[\begin{array}{l}
1 \\
1 \\
0 \\
0
\end{array}\right] \rightarrow\left[\begin{array}{l}
0 \\
0 \\
1 \\
1
\end{array}\right]\right)=1
$$

Thus, rule 3 satisfies detailed balance. Note that this also verifies detailed balance of rule 12 . Checking the remaining rules, we find that $L B_{1}$ satisfies detailed balance.

Note that while a lattice gas method may not satisfy detailed balance it may sacisfy semi-detailed balance.

Definition 2.16 (Semi-Détailed Balance). Let $\mathcal{S}(\alpha \rightarrow \beta)=\sum_{j=0}^{J} \delta^{j} \mathcal{S}^{(j)}(\alpha \rightarrow$ $\beta$ ) be the state transition function of a lattice gas method. If

$$
\sum_{\alpha \in \mathcal{B}^{d}} \mathcal{S}^{(0)}(\alpha \rightarrow \beta)=1 \quad \forall \beta \in \mathcal{B}^{d}
$$

then we say that the lattice method satisfies semi-detailed balance.

Definition 2.17 (Quasi-Detailed Balance). $L c^{\prime} \mathcal{S}(\alpha \rightarrow \beta)$ be the state transition function of a lattice gas method. If

$$
\sum_{\beta \in \mathcal{B}^{d}} \beta \mathcal{S}(\alpha \rightarrow \beta)=\sum_{\beta \in \mathcal{B}^{d}} \beta \mathcal{S}(\beta \rightarrow \alpha) \forall \alpha \in \mathcal{B}^{d}
$$

then we say that the lattice method satisfies quasi-detailed balance.

Note that detailed balance is a special case of semi-detailed balance, for

$$
\begin{aligned}
\mathcal{S}(\alpha \rightarrow \beta) & =\mathcal{S}(\beta \rightarrow \alpha) \forall \alpha, \beta \in \mathcal{B}^{d} \\
\Rightarrow \sum_{\alpha} \mathcal{S}(\alpha \rightarrow \beta) & =\sum_{\alpha} \mathcal{S}(\beta \rightarrow \alpha) \\
& =1 \forall \beta \in \mathcal{B}^{d},
\end{aligned}
$$


by renaming indicies and since all transition probabilities are normalized so that $\sum_{\beta \in \mathcal{B}^{d}} \mathcal{S}(\alpha \rightarrow \beta)=1 \quad \forall \alpha \in \mathcal{B}^{d}$.

Example (A Lattice Method with Semi-Detailed Balance without Detailed Balance).

Consider a lattice method on a two-dimensional square lattice (the lattice used in $\left.L B_{1}\right)$. The collision rules are defined as follows:

- If a lattice node has exactly one particle, moving in, say, direction $k$, then its new direction is $k+1$ (evaluated modulo 4$)$.

- In all other cases, particles do not interact, passing through each other, i.e., the node's state does not change.

The rules are summarized in Table 2.2. By examining the table one finds that all rules, excepting rules $1,2,4$, and 8 (the single-particle collision rules), satisfy detailed balance. Rules $1,2,4$, and 8 individually satisfy semi-detailed balance, however. Consider rule 1 , in which

$$
\alpha=\left[\begin{array}{l}
0 \\
0 \\
0 \\
1
\end{array}\right], \quad \beta=\left[\begin{array}{l}
1 \\
0 \\
0 \\
0
\end{array}\right],
$$

and $\mathcal{S}(\alpha \rightarrow \beta)=1$. But $\mathcal{S}(\beta \rightarrow \alpha)=0$, i.e., state $\beta$ never makes a transition to state $\alpha$. However, semi-detailed balance is satisfied, for with $\beta$ fixed as above, $\sum_{\zeta \in \mathcal{B}^{4}} \mathcal{S}(\zeta \rightarrow \beta)=\mathcal{S}(\alpha \rightarrow \beta)=1$. Checking the other non-detailed balance rules $(2,4$, and 8$)$, it can be verified that they satisfy semi-detailed balance. Thus, on the whole the lattice gas method satisfies semi-detailed balance.

Whether a lattice gas method satisfies detailed or semi-detailed balance is important for one method of determining its equilibrium behavior. We will see this in 
Table 2.2: Coilision Rules for an Example of Semi-Detailed Balance without Detailed Balance.

\begin{tabular}{|c|c|c|c|c|c|c|c|c|c|}
\hline \multirow[t]{2}{*}{ Rule } & \multicolumn{4}{|c|}{$\alpha$} & \multicolumn{4}{|c|}{$\beta$} & \multirow{2}{*}{$\mathcal{S}(\alpha \rightarrow \beta)$} \\
\hline & $\overline{\alpha_{0}}$ & $\bar{\alpha}_{1}$ & $\alpha_{2}$ & $\alpha_{3}$ & $\beta_{0}$ & $\beta_{1}$ & $\overline{\beta_{2}}$ & $\overline{\beta_{3}}$ & \\
\hline 0 & 0 & 0 & 0 & 0 & 0 & 0 & 0 & 0 & 1 \\
\hline 1 & 0 & 0 & 0 & 1 & 1 & 0 & 0 & 0 & 1 \\
\hline 2 & 0 & 0 & 1 & 0 & 0 & 0 & 0 & 1 & 1 \\
\hline 3 & 0 & 0 & 1 & 1 & 0 & 0 & 1 & 1 & 1 \\
\hline 4 & 0 & 1 & 0 & 0 & 0 & 0 & 1 & 0 & 1 \\
\hline 5 & 0 & 1 & 0 & 1 & 0 & 1 & 0 & 1 & 1 \\
\hline 6 & 0 & 1 & 1 & 0 & 0 & 1 & 1 & 0 & 1 \\
\hline 7 & 0 & 1 & 1 & 1 & 0 & 1 & 1 & 1 & 1 \\
\hline 8 & 1 & 0 & 0 & 0 & 0 & 1 & 0 & 0 & 1 \\
\hline 9 & 1 & 0 & 0 & 1 & 1 & 0 & 0 & 1 & 1 \\
\hline 10 & 1 & 0 & 1 & 0 & 1 & 0 & 1 & 0 & 1 \\
\hline 11 & 1 & 0 & 1 & 1 & 1 & 0 & 1 & 1 & 1 \\
\hline 12 & 1 & 1 & 0 & 0 & 1 & 1 & 0 & 0 & 1 \\
\hline 13 & 1 & 1 & 0 & 1 & 1 & 1 & 0 & 1 & 1 \\
\hline 14 & 1 & 1 & 1 & 0 & 1 & 1 & 1 & 0 & 1 \\
\hline 15 & 1 & 1 & 1 & 1 & 1 & 1 & 1 & 1 & 1 \\
\hline
\end{tabular}


Section 2.2.

The collision operator, $\mathcal{C}$, is defined in terms of the state transition function, $\mathcal{S}$, as follows:

Definition 2.18 (COLlision Operator). Let a lattice gas method on a regular lattice with d directions in $p$-dimensional space be given. Let its state transition function, $\mathcal{S}: \mathcal{B}^{d} \times \mathcal{B}^{d} \rightarrow[0,1]$, and occupation numbers $\hat{\mathbf{n}}=\hat{\mathbf{n}}(\vec{\imath} ; n) \in \mathcal{B}^{d}$, be given. Then the collision operator, $\mathcal{C}: \mathcal{B}^{d} \rightarrow \mathcal{B}^{d}$, of the lattice gas method is defined by

$$
\mathcal{C}(\hat{\mathbf{n}}) \equiv \sum_{\alpha, \beta \in \mathcal{B}^{d}} \mathcal{S}(\alpha \rightarrow \beta)(\beta-\alpha) \hat{\mathbf{n}}^{\alpha} \overline{\hat{\mathbf{n}}}^{\bar{\alpha}}
$$

The $k^{\text {th }}$ component of the collision operator is

$$
\mathcal{C}_{k}(\hat{\mathbf{n}})=\sum_{\alpha, \beta \in \mathcal{B}^{d}} \mathcal{S}(\alpha \rightarrow \beta)\left(\beta_{k}-\alpha_{k}\right) \hat{\mathbf{n}}^{\alpha} \overline{\hat{\mathbf{n}}}^{\bar{\alpha}}
$$

where

$$
\begin{aligned}
& \mathcal{S}(\alpha \rightarrow \beta) \text { is the probability of a transition from state } \alpha \text { to state } \beta, \\
& \left(\beta_{k}-\alpha_{k}\right) \in\{-1,0,1\} \text { is the correction for direction } k, \\
& \hat{\mathbf{n}}^{\alpha} \overline{\hat{\mathbf{n}}}^{\bar{\alpha}}= \begin{cases}1, & \text { if } \hat{\mathbf{n}}(\vec{\imath} ; n)=\alpha \\
0, & \text { otherwise }\end{cases}
\end{aligned}
$$

To see the last statement, look back at Eq. 2.2 and use the fact that $\hat{n}_{k} \in\{0,1\}$.

We can now formally define a lattice gas method:

Definition 2.19 (Lattice Gas Method). A lattice gas method is defined as a system that satisfies Eq. 2.5 with the advection operator, $\mathcal{A}$, and the collision operator, $\mathcal{C}$, as defined in Definitions 2.9 and 2.18 , respectively. Let $\mathcal{S}$ be the state

\footnotetext{
${ }^{7}$ The usefulness of quasi-detailed balance is under investigation for guaranteeing certain properties of the linearized collision operater.
} 
transition function of a lattice gas method. If the range of $\mathcal{S}$ is $\mathcal{B}$, then we say that the lattice gas method is deterministic. If the range of $\mathcal{S}$ is $[0,1]$ and not $\mathcal{B}$, then we say that the lattice gas method is stochastic or probabilistic.

DEFINITION 2.20 (ROTATION INVARIANCE). On a two-dimensional lattice, a collision operator is said to be rotation invariant if $\forall \hat{\mathbf{n}} \in \mathcal{B}^{d}, \forall l \in\{0,1, \ldots, d-1\}$,

$$
\mathcal{C}\left(\left[\hat{n}_{k+1}\right]_{k=0}^{d-1}\right)=\mathcal{C}\left(\left[\hat{n}_{k+1+1}\right]_{k=0}^{d-1}\right)
$$

in which all indices are evaluated modulo $d$.

\section{Example ( $L B_{1}$ : Collision Operator).}

We can write the collision operator for $L B_{1}$ by examining its collision rules in Table 2.1. The collision operator is a summation over all the collision rules and with $\hat{\mathbf{n}}=\hat{\mathbf{n}}(\vec{\imath} ; n)$ may be written:

$$
\mathcal{C}(\hat{\mathbf{n}})=\sum_{\alpha, \beta \in \mathcal{B}^{d}} \mathcal{S}(\alpha \rightarrow \beta)(\beta-\alpha) \hat{\mathbf{n}}^{\alpha} \overline{\hat{\mathbf{n}}}^{\bar{\alpha}}=\sum_{\alpha, \beta \in \mathcal{B}^{\alpha}} \mathcal{S}(\alpha \rightarrow \beta)(\beta-\alpha) \hat{\mathbf{n}}^{\alpha} \overline{\hat{\mathbf{n}}}^{\bar{\alpha}},
$$

Each rule in Table 2.1 contributes one term to the summation. We will look in detail how one of these terms is generated, and then forgo the details for the rest of the terms. Consider rule 9 (denoted $R_{9}$ ) in Table 2.1 We want to determine its contribution to the collision operator summation. Well, in rule 9

$$
\alpha=\left[\begin{array}{l}
1 \\
0 \\
0 \\
1
\end{array}\right], \quad \beta=\left[\begin{array}{l}
0 \\
1 \\
1 \\
0
\end{array}\right],
$$

and

$$
\mathcal{S}(\alpha-j)=1
$$


Then

$$
\begin{aligned}
& (\beta-\alpha)=\left(\left[\begin{array}{l}
0 \\
1 \\
1 \\
0
\end{array}\right]-\left[\begin{array}{l}
1 \\
0 \\
0 \\
1
\end{array}\right]\right)=\left[\begin{array}{l}
-1 \\
+1 \\
+1 \\
-1
\end{array}\right], \\
& \dot{\mathbf{n}}^{\alpha}=\hat{\mathfrak{n}}\left[\begin{array}{llll}
1, & 0, & 0, & 1
\end{array}\right]^{T}=\hat{n}_{0}^{1} \hat{n}_{1}^{0} \hat{n}_{2}^{0} \hat{n}_{3}^{1}=\hat{n}_{0} \hat{n}_{3}, \\
& \overrightarrow{\hat{\mathbf{n}}}^{\bar{\alpha}}=\overline{\left[\begin{array}{llll}
1, & 0, & 0, & 1
\end{array}\right]^{T}}=(1-\hat{\mathbf{n}})\left[\begin{array}{llll}
0, & 1, & 1, & 0
\end{array}\right]^{T} \\
& =\left(1-\hat{n}_{0}\right)^{0}\left(1-\hat{n}_{1}\right)^{1}\left(1-\hat{n}_{2}\right)^{1}\left(1-\hat{n}_{3}\right)^{0} \\
& =\left(1-\hat{n}_{1}\right)\left(1-\hat{n}_{2}\right) \text {. }
\end{aligned}
$$

And so, 8

$$
\begin{aligned}
\hat{\mathbf{n}}^{\alpha} \overline{\hat{\mathbf{n}}}^{\bar{\alpha}} & =\hat{\mathbf{n}}\left[\begin{array}{llll}
1, & 0, & 0, & 1
\end{array}\right]^{T} \overline{\overline{\hat{\mathbf{n}}}} \overline{\left[\begin{array}{llll}
1, & 0, & 0, & 1
\end{array}\right]^{T}} \\
& =\hat{n}_{0}\left(1-\hat{n}_{1}\right)\left(1-\hat{n}_{2}\right) \hat{n}_{3} \\
& =\hat{n}_{0} \overline{\hat{n}_{1}} \overline{\hat{n}_{2}} \hat{n}_{3},
\end{aligned}
$$

which is a scalar, as expected. Then the term contributed by rule 9 to the collision operator is

$$
\mathcal{S}(\alpha \rightarrow \beta)(\beta-\alpha) \hat{\mathbf{n}}^{\alpha} \overline{\hat{\mathbf{n}}}^{\bar{\alpha}}=\left[\begin{array}{c}
-1 \\
+1 \\
+1 \\
-1
\end{array}\right] \hat{n}_{0} \overline{\hat{n}_{1}} \overline{\hat{n}_{2}} \hat{n}_{3} .
$$

The 0th component of the above is $-\hat{n}_{0} \overline{\hat{n}_{1}} \overline{\hat{n}_{2}} \hat{n}_{3}$. Rule 9 contributes this term to the Oth component of the collision operator, $\mathcal{C}_{0}(\hat{\mathbf{n}})$, in the following: ${ }^{9}$

$$
\mathcal{C}_{0}(\hat{\mathbf{n}})=
$$

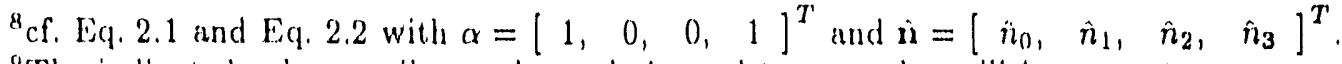

gre indicated rule contributes the underbraced term to the collision operator. 


$$
\underbrace{+\overline{n_{0}} \overline{n_{1}} \hat{n}_{2} \hat{n}_{3}}_{\text {ivule } 3} \underbrace{+\overline{n_{0}} \hat{n}_{1} \overline{n_{2}} \hat{n}_{3}}_{\text {Rule } 5} \underbrace{+\overline{n_{0}} \hat{n}_{1} \hat{n}_{2} \overline{n_{3}}}_{\text {Rule } 6} \underbrace{-\hat{n}_{0} \overline{n_{1}} \overline{n_{2}} \hat{n}_{3}}_{\text {Rule } 9} \underbrace{-\hat{n}_{0} \overline{n_{1}} \hat{n}_{2} \overline{n_{3}}}_{\text {Rule } 10} \underbrace{-\hat{n}_{0} \hat{n}_{1} \overline{\hat{n}_{2}} \overline{n_{3}}}_{\text {Rule } 12} .
$$

The other terms (besides the one contributed by rule 9) in the above san be determined directly from the collision rules in the same way as outlined for rule 9 . Because $L B_{1}$ is rotation invariant, we can write

$$
\begin{aligned}
\mathcal{C}_{k}(\hat{\mathbf{n}})= & +\overline{n_{k}} \overline{n_{k+1}} \hat{n}_{k+2} \hat{n}_{k+3}+\bar{n}_{k} \hat{n}_{k+1} \overline{n_{k+2}} \hat{n}_{k+3}+\bar{n}_{k} \hat{n}_{k+1} \hat{n}_{k+2} \overline{n_{k+3}} \\
& -\hat{n}_{k} \overline{n_{k+1}} \overline{\hat{n}_{k+2}} \hat{n}_{k+3}-\hat{n}_{k} \overline{n_{k+1}} \hat{n}_{k+2} \overline{\hat{n}_{k+3}}-\hat{n}_{k} \hat{n}_{k+1} \overline{\hat{n}_{k+2}} \overline{\hat{n}_{k+3}},
\end{aligned}
$$

in which the indices are evaluated modulo $d=4$. Alternatively, we could go through the collision rules and explicitly determine each component of the collision operator; we would find that we could write it as in Eq. 2.7. We can write the collision operator as a vector by

$$
\mathcal{C}(\hat{\mathbf{n}})=\left[\mathcal{C}_{k}(\hat{\mathbf{n}})\right] .
$$

\subsubsection{Lattice Boltzmann Methods}

Definition 2.21 (Mean Occupation Numbers). Denote the ensemble average of the occupation number for direction $k$ by

$$
\left(n_{k}\right)_{i}^{n}=\left\langle\left(\hat{n}_{k}\right)_{i}^{n}\right\rangle
$$

Then $\left(n_{k}\right)_{\mathfrak{r}}^{n} \in[0,1]$ is called the mean occupation number in direction $k$ at $(\vec{\imath} ; n)$. The vector of mean occupation numbers at node $\vec{\imath}$ and time step $n$ is denoted

$$
\mathbf{n}_{\mathfrak{r}}^{n}=\left[\left(n_{k}\right)_{\mathfrak{i}}^{n}\right]_{k=0}^{d-1} \in[0,1]^{d} .
$$

The microdynamical equation for the mean occupation numbers is

$$
\mathcal{A} \mathrm{n}_{\mathrm{r}}^{n}=\mathrm{n}_{\mathrm{r}}^{n}+\left\langle\mathcal{C}\left(\hat{\mathbf{n}}_{\mathrm{r}}^{n}\right)\right\rangle
$$


Assuming that the expectod value, i.e, the ensemble average operator, passes through the nonlinearities in the collision operator, we may write

$$
\mathcal{C}\left(\mathbf{n}_{\uparrow}^{n}\right)=\left\langle\mathcal{C}\left(\hat{\mathbf{n}}_{\uparrow}^{n}\right)\right\rangle
$$

DFfinition 2.22 (LATTiCe Boltzmann Approximation), The Lattice Boltzmann Approximation to a lattice gas method is

$$
\mathcal{A} \mathrm{n}_{\uparrow}^{n}=\mathrm{n}_{\uparrow}^{n}+\mathcal{C}\left(\mathrm{n}_{\tilde{\imath}}^{n}\right){ }^{10}
$$

We refer to $\mathcal{C}$ as the lattice Boltzmann collision operator Under this approximation, we refer to the $\mathbf{n}_{\uparrow}^{n}$ as the occupation numbers. The extent to which the Lattice Boltzmann Approximation to a lattice gas method holds depends on the underlying statistical properties of the lattice gas method. Specifically, the covariances ${ }^{11}$ must be negligible.

We will using an ordering the lattice Boltzmann collision operator in later analysis. Let $\delta$ such that $0<\delta \ll 1$ be given and consider a fixed vector, $\mathrm{n} \in[0,1]^{d}$, of occupation numbers. The we will assume that there exists coefficients, $\mathcal{C}^{(j)}$, such that

$$
\mathcal{C}(\mathbf{n})=\sum_{j=0}^{J} \delta^{j} \mathcal{C}^{(j)}(\mathbf{n}),
$$

for some $J \geq 0$. This expansion is based on the Eq. 2.6, the expansion for $\mathcal{S}$. A dependency of the collision operator on $\delta$ can produce a method that models nonlinear advection, the nonlinearity being introduced as a consequence of the Fermi Exclusion Principle. For the methods studied herein, we assume that $J \leq 1$. In the absence of advection, we write $\mathcal{C}(\mathbf{n})=\mathcal{C}^{(0)}(\mathbf{n})$; such is the case for $L B_{1}$. (See Sections 3.2 and

\footnotetext{
${ }^{10}$ This equation, in reference to lattice Boltzmann methods (see Definition 2.23 ), is called the Lattice Boltzmann Equation.

${ }^{1}$ See Definition 2.5 regarding covariances. Also, see the example in Section 3.2 , in particular, Section 3.2.3, for an explicit representation of the covariances in a lattice gas method.
} 
3.3 for cases in which $J=1$.) Note that $\mathcal{C}^{(0)}(\mathrm{n})$ is referred to as the $\mathcal{O}[1]$ contribution of the collision operator.

Example (LB $B_{1}$ Lattice Boltzmann Collision Operator).

The lattice Boltzmann collision operator for $L B_{1}$ is given by

$$
\begin{aligned}
\mathcal{C}_{k}(\mathbf{n})= & +\pi_{k} \overline{n_{k+1}} n_{k+2} n_{k+3}+\pi_{k} n_{k+1} \overline{n_{k+2}} n_{k+3}+\pi_{k} n_{k+1} n_{k+2} \overline{n_{k+3}} \\
& -n_{k} \overline{n_{k+1}} \overline{n_{k+2}} n_{k+3}-n_{k} \overline{n_{k+1}} n_{k+2} \overline{n_{k+3}}-n_{k} n_{k+1} \overline{n_{k+2}} \pi_{k+3}
\end{aligned}
$$

and remains valid in the Lattice Boltzmann Approximation to the extent that the covariances may be neglected, i.e., that the ensemble average operator passes through the nonlinearities in the collision operator. In vector notation, we write

$$
\mathcal{C}(\mathbf{n})=\left[\mathcal{C}_{k}(\mathbf{n})\right]_{k=0}^{\jmath^{3}}
$$

Definition 2.23 (LatTice Boltzmann Metiod). Let the occupation numbers $\mathbf{n}_{\tilde{r}}^{n} \in[0,1]^{d}$ evolve according to Eq. 2.8. Then a method satisfying microdynamical equation Eq. 2.8 is called a lattice Boltzmann method. We refer to Eq. 2.8 as the Lattice Boltzmann Equation.

\section{Discussion}

The occupation numbers in Eq. 2.8 are analogous to particle density functions in the Boltzmann equation of hydrodynamics as can be found in, for example, [4]. We will see later that lattice Boltzmann methods are finite difference methods. A major difference between lattice Boltzmann methods and typical finite difference methods is that in the latter, the hydrodynamical equations, i.e., the partial differential equations, are differenced, whereas in the former the hydrodynamical equations are not directly 
differenced, rather the Boltzmann equation is differenced. The differenced quantities relate in a special way to the solution of the hydrodynamical equations.

Note that the definition of lattice Boltzmann method may be extended by dropping the Fermi Exclusion Principle so that $\mathrm{n}_{\mathrm{r}}^{n} \in[0, \infty)^{d}$. The same microdynamical equation applies, i.e., Eq. 2.8; however, the collision operator is then defined by

$$
\begin{aligned}
\mathcal{C}(\mathbf{n}(\vec{\imath} ; n)) & \left.=\sum_{\alpha, \beta \in \mathcal{B}^{d}}(\beta-\alpha) \mathcal{S}_{(}^{\prime} \alpha \rightarrow \beta\right)(\mathbf{n}(\vec{\imath} ; n))^{\alpha} \\
& =\sum_{\alpha, \beta \in \mathcal{B}^{d}}(\beta-\alpha) \mathcal{S}(\alpha \rightarrow \beta) \prod_{k=0}^{d-1}\left(n_{k}(\vec{\imath} ; n)\right)^{\alpha_{k}},
\end{aligned}
$$

in which $\left(n_{k}(\vec{r} ; n)\right)^{0}$ is defined to be 1 for all $n_{k}(\vec{r} ; n) \in \mathcal{R}$. Note that a lattice Boltzmann method without the Fermi Exclusion Principle is not a Lattice Boltzmann Approximation to a lattice gas method, for without that principle the occupation numbers are not restricted to the interval $[0,1]$.

\section{Example (A Lattice Method Without the Fermi Exclusion Principle).}

Dropping the Fermi Exclusion Principle in $L B_{1}$ yields the collision operator,

$$
\mathcal{C}(\mathbf{n})=\left[\mathcal{C}_{k}(\mathbf{n})\right]_{k=0}^{3},
$$

where

$$
\mathcal{C}_{k}(\mathbf{n})=n_{k+2} n_{k+3}+n_{k+1} n_{k+3}+n_{k+1} n_{k+2}-n_{k} n_{k+3}-n_{k} n_{k+2}-n_{k} n_{k+1},
$$

the indices being evaluated modulo 4. Note that the lattice Boltzmann method,

$$
\mathcal{A} \mathbf{n}_{i}^{n}=\mathbf{n}_{\uparrow}^{n}+\mathcal{C}\left(\mathbf{n}_{\tilde{\imath}}^{n}\right)
$$

requires fewer arithmetic operations than in the corresponding lattice Boltzmann method with the Fermi Exclusion Principle, i.e., LB 1 (cf. Eq. 2.10, the collision operator for $\left.L B_{1}\right)$. 
Subsequent analysis herein pertains to lattice Boltzmann methods with the Fermi Exclusion Principle (tisn the analysis applies to their counterpart lattice gas methods under certain statistical assertions). Most of the analysis can be easily and straightforwardly extended to lattice Boltzmann methods without the Ferni Exclusion Principle by using occupation numbers $\mathbf{n} \in[0, \infty)^{d}$ and a collision operator as defined in Eq. 2.11, with the same definitions for the various types o: balance, e.g., semi-detailed balance.

\subsection{Equiiibrium Analysis}

DeFinition 2.24 (EQUILIBRIUM). If for $\mathbf{n}^{(0)} \in[0,1]^{d}, \mathcal{C}^{(0)}\left(\mathbf{n}^{(0)}\right)=0$, then we say that $\mathbf{n}^{(0)}$ is an equilibrium solution of the Lattice Boltzmann Equation, Eq. 2.8.

This section provides and discusses two methods for determining equilibrium solutions of the Lattice Boltzmann Equation, Eq. 2.8. One approach is via a theorem for lattice methods satisfying semi-detailed balance. The other approach is more direct and applies even in the absence of semi-detailed balance. It is, however, somewhat more cumbersome to use in lattice methods with large phase spaces, i.e., large $d$. But for lattice methods not satisfying semi-detailed balance, one generally resorts to the alternative approach. Once having obtained the algebraic form of equilibrium solution, it is used to calculate the linearized collision operator of the lattice method, which is employed to generate the discrete Chapman-Enskog expansion. First, we introduce the direct method, then the one requiring semi-detailed balance. 


\subsubsection{Determining Equilibria Directly}

The simplest and most direct method for determining the equilibria of a lattice method is to set the $\mathcal{O}[1]$ contribution of the collision operator, $\mathcal{C}^{(0)}(\mathbf{n})$, to zero. This produces $d$ nonlinear equations and $d$ unknowns. The equations may be manipulated so that each unknown is in terms of the other unknowns.

Example (LB 1 : Equilibria via Direct Niethod).

Consider setting the collision operator (as in Eq. 2.10) to zero. Note that the collision operator is $\mathcal{O}[1]$, i.e., $\mathcal{C}(\mathbf{n})=\mathcal{C}^{(0)}(\mathbf{n})$. Then $\mathcal{C}(\mathbf{n})=\mathbf{0}$ implies that $\mathcal{C}_{0}(\mathbf{n})=\mathcal{C}_{1}(\mathbf{n})$. After some algebraic manipulation, we find $n_{0}=n_{1}$. Similarly,

$$
\begin{aligned}
& \mathcal{C}_{0}(\mathbf{n})=\mathcal{C}_{2}(\mathbf{n}) \Rightarrow n_{0}=n_{2}, \\
& \mathcal{C}_{0}(\mathbf{n})=\mathcal{C}_{3}(\mathbf{n}) \Rightarrow n_{0}=n_{3} .
\end{aligned}
$$

Hence, $n_{0}=n_{1}=n_{2}=n_{3} \equiv u$ and the equilibrium solution has the form

$$
\mathbf{n}^{(0)}=\left[\begin{array}{l}
u \\
u \\
u \\
u
\end{array}\right] \text {. }
$$

\subsubsection{Equilibrium Theorem}

Another technique for determining equilibria of a lattice gas method is through the following theorem. This theorem applies to lattice methods that satisfy semi-detailed balance.

Theorem 2.25 (Equilibrium Theorem). Let a lattice method satisfying semi-detailed balance and upholding the Fermi Exclusion Principle be given with collision operator, $\mathcal{C}:[0,1]^{d} \rightarrow[0,1]^{d}$ with expansion, Eq. 2.9, and state transition 
function, $\mathcal{S}(\alpha \rightarrow \beta)$, with expansion, Eq. 2.6. Then for $\mathbf{n} \in(0,1)^{d}, \mathcal{C}^{(0)}(\mathbf{n})=0$ if and only if $\mathbf{n}^{\alpha} \overline{\mathbf{n}}=\mathbf{n}^{\beta} \overline{\mathbf{n}}^{\bar{\beta}} \forall \alpha, \beta \in \mathcal{B}^{d}$ such that $\mathcal{S}^{(0)}(\alpha \rightarrow \beta) \neq 0$.

First, we introduce and prove some lemmas, after which we prove the theorem. The lemmas (and the subsequent proof of the theorem) use an auxiliary function, $s: \mathcal{R}^{d} \rightarrow \mathcal{R}$, defined by

$$
s(\mathbf{n})=-(\mathbf{1}-\mathbf{n}) \cdot \log (\mathbf{1}-\mathbf{n})-\mathbf{n} \cdot \log \mathbf{n},
$$

Now, let $s^{\prime}(\mathbf{n})$ denote the gradient,

$$
s^{\prime}(\mathbf{n}) \equiv\left[\frac{\partial}{\partial n_{k}} s(\mathbf{n})\right]_{k=0}^{d-1} .
$$

Then we have vector

$$
\begin{aligned}
\boldsymbol{s}^{\prime}(\mathbf{n}) & =\log (\mathbf{1}-\mathbf{n})-\log \mathbf{n} \\
& =\log \left(\frac{\mathbf{1}-\mathbf{n}}{\mathbf{n}}\right) \\
& =-\log \left(\frac{\mathbf{n}}{\mathbf{1}-\mathbf{n}}\right) \\
& =-\left[\log \left(\frac{n_{k}}{1-n_{k}}\right)\right]_{k=0}^{d-1}
\end{aligned}
$$

and

$$
s^{\prime \prime}(\mathbf{n}) \equiv\left[\frac{\partial^{2}}{\partial n_{k} \partial n_{l}} s_{k, l}\right]_{k, l=0}^{d-1}
$$

where

$$
s_{k, l}= \begin{cases}\frac{1}{n_{k}\left(1-n_{l}\right)}, & k=l \\ 0, & \text { otherwise }\end{cases}
$$

Note that for $\mathbf{n} \in(0,1)^{d}$, the $s^{\prime \prime}(\mathbf{n})$ is negative definite. Hence, $s(\mathbf{n})$ is concave downward on the interval $(0,1)^{d}$. We now proceed with the statement of the lemmas. 
LEMMA 2.26. Let $\alpha, \beta \in \mathcal{B}^{d}$. Then $(\beta-\alpha) \cdot s^{\prime}(\mathbf{n})=\log \left(\frac{\mathbf{n}^{\alpha} \overline{\mathbf{n}}^{\bar{\alpha}}}{\mathbf{n}^{\beta} \overline{\mathbf{n}}^{\bar{\beta}}}\right)$.

Proof. Recalling that $\overline{\mathbf{n}}=\mathbf{1}-\mathbf{n}$ with $\mathbf{1} \in \mathcal{R}^{d}$ denoting a vector of ones, we have

$$
\begin{aligned}
(\beta-\alpha) \cdot s^{\prime}(\mathbf{n}) & =-\sum_{k=0}^{d-1}\left(\beta_{k}-\alpha_{k}\right) \log \frac{n_{k}}{1-n_{k}} \\
& =-\log \prod_{k=0}^{d-1}\left(\frac{n_{k}}{1-n_{k}}\right)^{\left(\beta_{k}-\alpha_{k}\right)} \\
& =\log \left(\frac{\mathbf{n}}{1-\mathbf{n}}\right)^{(\alpha-\beta)} \\
& =\log \mathbf{n}^{(\alpha-\beta)}-\log \overline{\mathbf{n}}^{(\alpha-\beta)} \\
& =\log \mathbf{n}^{\alpha} \overline{\mathbf{n}}^{\bar{\alpha}}-\log \mathbf{n}^{\beta} \overline{\mathbf{n}}^{\bar{\beta}}-\log \overline{\mathbf{n}}^{1}+\log \overline{\mathbf{n}}^{1} \\
& =\log \frac{\mathbf{n}^{\alpha} \overline{\mathbf{n}}^{\bar{\alpha}}}{\mathbf{n}^{\beta} \overline{\mathbf{n}}^{\bar{\beta}}} .
\end{aligned}
$$

A result of semi-detailed balance is the following:

LEMMA 2.27. $\sum_{\alpha, \beta} \mathcal{S}^{(0)}(\alpha \rightarrow \beta)\left(\mathbf{n}^{\beta} \overline{\mathbf{n}}^{\bar{\beta}}-\mathbf{n}^{\alpha} \overline{\mathbf{n}}^{\bar{\alpha}}\right)=0$.

Proof. Semi-detailed balance and renaming indices gives

$$
\begin{aligned}
& \sum_{\alpha, \beta \in \mathcal{B}^{\alpha}} \mathcal{S}^{(0)}(\alpha \rightarrow \beta)\left(\mathbf{n}^{\beta} \overline{\mathbf{n}}^{\bar{\beta}}-\mathbf{n}^{\alpha} \overline{\mathbf{n}}^{\bar{\alpha}}\right) \\
& =\sum_{\beta} \mathbf{n}^{\beta} \overline{\mathbf{n}}^{\bar{\beta}} \sum_{\alpha} \mathcal{S}^{(0)}(\alpha \rightarrow \beta)-\sum_{\alpha, \beta} \mathcal{S}^{(0)}(\alpha \rightarrow \beta) \mathbf{n}^{\alpha} \overline{\mathbf{n}}^{\bar{\alpha}} \\
& =\sum_{\alpha, \beta} \mathcal{S}^{(0)}(\alpha \rightarrow \beta) \mathbf{n}^{\alpha} \overline{\mathbf{n}}^{\bar{\alpha}}-\sum_{\alpha, \beta} \mathcal{S}^{(0)}(\alpha \rightarrow \beta) \mathbf{n}^{\alpha} \overline{\mathbf{n}}^{\bar{\alpha}} \\
& =0
\end{aligned}
$$

LemMA 2.28. Let $f(x, y)=x \log (x / y)+y-x$. Then for any $(x, y) \in \Omega=$ $(0, \infty) \times(0, \infty), f(x, y) \geq 0$ and $f(x, y)=0$ if and only if $x=y$.

Proof. We look at the global minimum of $f$ on $\Omega$, and we show that the global minimum is 0 is achieved for $(x, y)$ such that $x=y$. First, we show that the only local 
Table 2.3: The Sign of the First Partial Derivatives of $f(x, y)$.

\begin{tabular}{||c||c|c||}
\hline \hline & $\operatorname{sign}\left(f_{x}(x, y)\right)$ & $\operatorname{sign}\left(f_{y}(x, y)\right)$ \\
\hline \hline$x<y$ & - & + \\
\hline$x>y$ & + & - \\
\hline \hline
\end{tabular}

extrema, which are zero, are at those points in $\Lambda \equiv\{(x, y) \mid x=y\}$. Then we show that on the boundary of $\Omega$, i.e., $\partial \Omega, f(x, y)$ is positive for $(x, y) \in\{(x, y) \mid x \neq y\} \subset$ $\partial \Omega$.

The critical points of $f(x, y)$ are found where $f_{x}(x, y)=f_{y}(x, y)=0$. Noting that $f_{x}(x, y)=\log (x / y)$ and $f_{y}(x, y)=1-x / y$, we find critical points at $(x, y) \in \Lambda$. To show that $(x, y) \in \Lambda$ is at a local minimum, we resort to examining $\operatorname{sign}\left(f_{x}(x, y)\right)$ and $\operatorname{sign}\left(f_{y}(x, y)\right)$ near $x=y$ since the second derivative test fails. The sign of the first partial derivatives of $f$ are exhibited in Table 2.3. The signs are such that indeed $(x, y) \in \Lambda$ gives a local minimum of $f(x, y)=0$. Hence, $x=y$ indeed gives a local minimum of $f(x, x)=0$.

Checking the boundaries of $\Omega$, we find

1. For fixed $x \neq 0 \lim _{y \rightarrow \infty} f(x, y)=\infty$ and $\lim _{y \rightarrow 0} f(x, y)=\infty$.

2. For fixed $y \neq 0 \lim _{x \rightarrow \infty} f(x, y)=\infty$ and $\lim _{x \rightarrow 0} f(x, y)=y$.

3. $\lim _{x \rightarrow 0} \lim _{y \rightarrow 0} f(x, y)=\infty$ and $\lim _{y \rightarrow 0} \lim _{x \rightarrow 0} f(x, y)=0$.

Then since $f(x, x)=0 \forall x \in(0, \infty)$ and the only local minima are at $(x, y) \ni x=y$, the global minimum of $f(x, y)$ is 0 and is achieved for $(x, y)$ such that $x=y$.

Figure 2.3 exhibits a graph of $f(x, y)$, substantiating the lemma. 


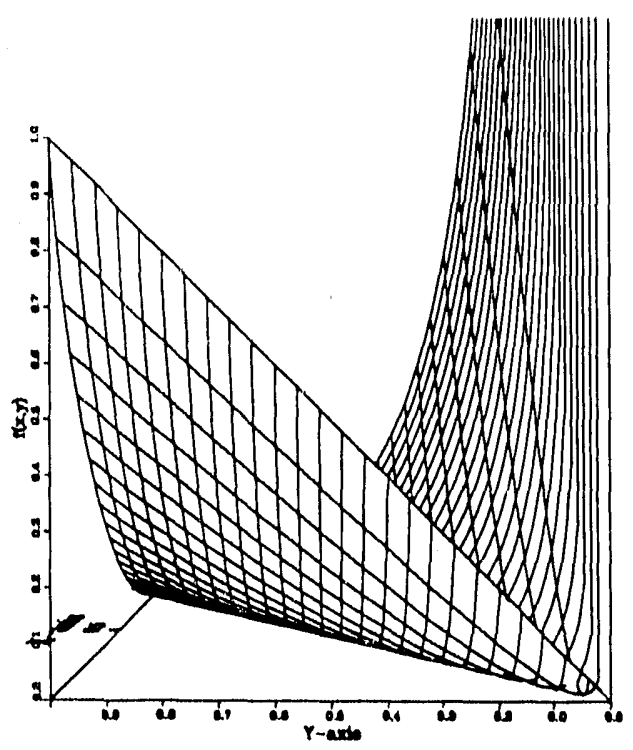

(a)

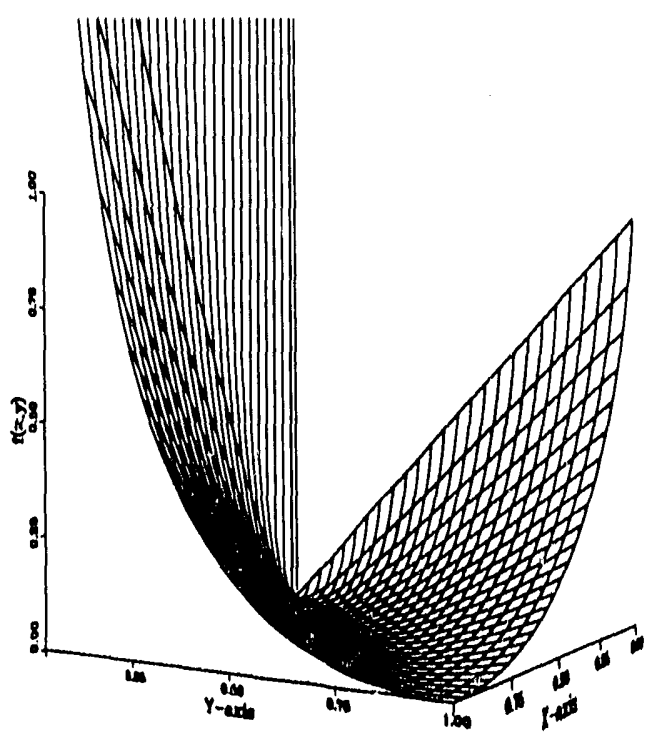

(b)

Figure 2.3: Graph of $f(x, y)=x \log (x / y)+y-x$ for $(x, y) \in[0,1] \times[0,1]$ from two perspectives. (a) Perspective is at $(x, y ; f(x, y))=(-3 / 2,1 / 2 ; 1 / 2)$; (b) perspective is at $(x, y ; f(x, y))=(3,2 ; 1 / 2)$. Note that the graphs are clipped as $f \rightarrow \infty$.

LEMMA 2.29. Let $f(x, y)$ be as in the previous lemma. Then

$$
s^{\prime}(\mathbf{n}) \cdot \mathcal{C}^{(0)}(\mathbf{n})=\sum_{\alpha, \beta} \mathcal{S}^{(0)}(\alpha \rightarrow \beta) f\left(\mathbf{n}^{\alpha} \overline{\mathbf{n}}^{\bar{\alpha}}, \mathbf{n}^{\beta} \overline{\mathbf{n}}^{\bar{\beta}}\right)
$$

Proof. The result is obtained by combining Lemmas 2.26 and 2.27 .

LEMMA 2.30. $s^{\prime}(\mathbf{n}) \cdot \mathcal{C}^{(0)}(\mathbf{n})=0$ if and only if $\mathbf{n}^{\alpha} \overline{\mathbf{n}}^{\bar{\alpha}}=\mathbf{n}^{\beta} \overline{\mathbf{n}}^{\bar{\beta}} \forall \alpha, \beta \in \mathcal{B}^{d} \ni$ $\mathcal{S}^{(0)}(\alpha \rightarrow \beta) \neq 0$.

Proof. Since $\mathcal{S}^{(0)}(\alpha \rightarrow \beta) \geq 0$,

$$
s^{\prime}(\mathbf{n}) \cdot \mathcal{C}^{(0)}(\mathbf{n})=\sum_{\alpha, \beta} \mathcal{S}^{(0)}(\alpha \rightarrow \beta) f\left(\mathbf{n}^{\alpha} \overline{\mathbf{n}}^{\bar{\alpha}}, \mathbf{n}^{\beta} \overline{\mathbf{n}}^{\bar{\beta}}\right)=0
$$

if and only if $\forall \alpha, \beta \ni \mathcal{S}^{(0)}(\alpha \rightarrow \beta)>0, f\left(\mathbf{n}^{\alpha} \overline{\mathbf{n}}^{\bar{\alpha}}, \mathbf{n}^{\beta} \overline{\mathbf{n}}^{\bar{\beta}}\right)=0$. Note that $\mathbf{n}^{\alpha} \overline{\mathbf{n}}^{\bar{\alpha}}>0$ and $\mathbf{n}^{\beta} \overline{\mathbf{n}}^{\bar{\beta}}>0$ since $\mathbf{n} \in(0,1)^{d}$. Then by Lemma $2.28, f\left(\mathbf{n}^{\alpha} \overline{\mathbf{n}}^{\bar{\alpha}}, \mathbf{n}^{\beta} \overline{\mathbf{n}}^{\bar{\beta}}\right)=0$ if and only if $\mathrm{n}^{\alpha} \overline{\mathbf{n}}^{\bar{\alpha}}=\mathrm{n}^{\beta} \overline{\mathbf{n}}^{\bar{\beta}}$. 
Proof of Theorem 2.25. In view of Lemma 2.30 it suffices to show that $s^{\prime}(\mathrm{n})$. $\mathcal{C}^{(0)}(\mathbf{n})=0$ if and only if $\mathcal{C}^{(0)}(\mathbf{n})=0$. Well, certainly $\mathcal{C}^{(0)}(\mathbf{n})=0$ implies $s^{\prime}(\mathbf{n})$. $\mathcal{C}^{(0)}(\mathbf{n})=0$. Now, assume $s^{\prime}(\mathbf{n}) \cdot \mathcal{C}^{(0)}(\mathbf{n})=0 \forall \mathbf{n} \in(0,1)^{d}$. Note that $s^{\prime}(\mathbf{n})=0$ if and only if $n_{k}=1 / 2 \forall k \in\{0,1, \ldots, d-1\}$; but, in this case, $\mathcal{C}^{(0)}(\mathbf{n})=0$ by semi-detailed balance. Thus, $\mathcal{C}^{(0)}(\mathbf{n})=0 \forall \mathbf{n} \in(0,1)^{d}$.

\subsubsection{Applying the Equilibrium Theorem}

We now apply the theorem from the previous subsection to determine possible equilibrium solutions of a lattice method satisfying semi-detailed balance. First, we will outline the steps, then apply them to our example method, $\mathrm{LB}_{1}$. The steps are as follows:

1. Verify that the lattice method satisfies semi-detailed balance, i.e., verify

$$
\sum_{\alpha} \mathcal{S}^{(0)}(\alpha \rightarrow \beta)=1
$$

2. For each $\alpha, \beta \ni \mathcal{S}^{(0)}(\alpha \rightarrow \beta)>0$ add $\mathbf{n}^{\alpha} \overline{\mathbf{n}}^{\bar{\alpha}}=\mathbf{n}^{\beta} \overline{\mathbf{n}}^{\bar{\beta}}$ to a system of (nonlinear) equations, by Theorem 2.25 .

3. Solve the system of equations and identify equilibrium solutions, if possible.

The following will help in applying Theorem 2.25.

Lemma 2.31. Let $\mathbf{n} \in(0,1)^{d}$ and define $\mathrm{g} \in(0,1)^{d}$ by $g_{k}=\frac{n_{k}}{1-n_{k}}$. Then for $l \neq k, g_{l}=g_{k}$ iff $n_{l}=n_{k}$.

Proof. Clearly,

$$
g_{l}=g_{k} \Leftrightarrow \frac{n_{l}}{1-n_{l}}=\frac{n_{k}}{1-n_{k}}
$$




$$
\begin{aligned}
& \Leftrightarrow \quad\left(1-n_{k}\right) n_{l}=\left(1-n_{l}\right) n_{k} \\
& \Leftrightarrow n_{l}-n_{k} n_{l}=n_{k}-n_{l} n_{k} \\
& \Leftrightarrow n_{l}=n_{k} .
\end{aligned}
$$

Example ( $L B_{1}$ : Equilibria via Theorem 2.25).

In $L B_{1}$, note that $\mathcal{S}(\alpha \rightarrow \beta)=\mathcal{S}^{(0)}(\alpha \rightarrow \beta)$. We determine the equilibria following the steps outlined above:

1. $L B_{1}$ satisfies detailed balance (and hence semi-detailed balance) as verified in Section 2.1 .2 (p. 24).

2. There are six collision rules in $\mathrm{LB}_{1}$ in which $\mathcal{S}^{(0)}(\alpha \rightarrow \beta)>0$. But because $L B_{1}$ satisfies detailed balance these rules generate only three unique equations. The system of equations is

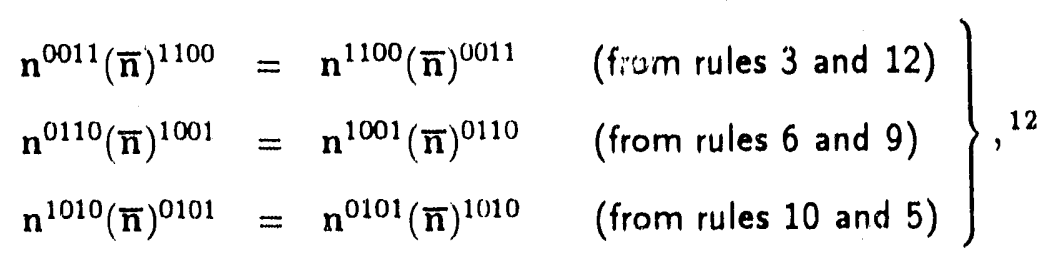

or

$$
\left.\begin{array}{l}
n_{2} n_{3} \overline{n_{0}} \overline{n_{1}}=n_{0} n_{1} \overline{n_{2}} \overline{n_{3}} \\
n_{1} n_{2} \overline{n_{0}} \overline{n_{3}}=n_{0} n_{3} \overline{n_{1}} \overline{n_{2}} \\
n_{0} n_{2} \overline{n_{1}} \overline{n_{3}}=n_{1} n_{3} \overline{n_{0}} \overline{n_{2}}
\end{array}\right\} .
$$

3. There are trivial solutions to Eq. 2.12 if we allow $n \in[0,1]^{d}$. They occur when various combinations of $n_{k}$ are either zero or one. Now, we return to

\footnotetext{
${ }^{12}$ Here, the superscripts are in shorthand vector exponent notation so that, e.g.,
}

$$
\mathbf{n}^{0011}=\mathbf{n}\left[\begin{array}{llll}
0, & 0, & 1 & 1
\end{array}\right]^{T} \text {. }
$$


the assumption, $\mathbf{n} \in(0,1)^{d}$, to determine non-trivial solutions. To help find the solutions, define $g_{k} \equiv n_{k} /\left(1-n_{k}\right)$, and write Eq. 2.12 in terms of the $g_{k}$. We then get

$$
\left.\begin{array}{ll}
g_{2} g_{3}=g_{0} g_{1} & \text { (a) } \\
g_{1} g_{2}=g_{0} g_{3} & \text { (b) } \\
g_{0} g_{2}=g_{1} g_{3} & \text { (c) }
\end{array}\right\} .
$$

Solve for $g_{0}$ in the first equation, (a), to get

$$
g_{0}=\frac{g_{2} g_{3}}{g_{1}}
$$

Substitute this for $g_{0}$ in the others, (b) and (c), to obtain (remember that each $g_{k}>0$ )

$$
\begin{aligned}
& g_{1}^{2}=g_{3}^{2} \Rightarrow g_{1}=g_{3} \\
& g_{2}^{2}=g_{1}^{2} \Rightarrow g_{2}=g_{1} .
\end{aligned}
$$

Substituting these for $g_{3}$ and $g_{2}$ in the second equation, (b), gives $g_{0}=g_{1}$. And in summary, we have

$$
g_{0}=g_{1}=g_{2}=g_{3} .
$$

Thrice by Lemma 2.31 , we have

$$
n_{0}=n_{1}=n_{2}=n_{3} \equiv u \text {. }
$$

Let $\mathbf{n}^{(0)}$ denote the non-trivial equilibrium solution. Then

$$
\mathbf{n}^{(0)}=\left[\begin{array}{l}
u \\
u \\
u \\
u
\end{array}\right] .
$$

Note that this is the same result found by the direct method of Section 2.2.1. 


\subsection{Discrete Chapman-Enskog Analysis}

This analysis recovers the partial differential equations that are consistent with a latlice method. These are known as the hydrodynamical equations of a lattice method. ${ }^{13}$ Also, toward proving convergence, the discrete Chapman-Enskog analysis is applied to verify formal consistency of lattice methods, as is shown in the next section. Buscally, the analysis involves matched asymptotic expansions: An asymptotic spatial-gradient expansion of the mean occupation numbers is combined with Taylor series expansions of the advection and collision operators for a lattice method to obtain the discrete Chapman-Enskog expansion. The analysis is accurate for lattice gas methods to the extent that the Lattice Boltzmann Approximation remains valids.

All expansions are in terms of a parameter, $\delta \ni 0<\delta \ll 1$ that is related to the size of the lattice though $\Delta x$ and $\Delta t$ and any $\mathcal{O}[\Delta x]$ advection biases. Different relationships between these parameters may result in differing sets of consistent equations. ${ }^{14}$ The following outlines the matched expansion analysis:

1. Expand the vector of occupation numbers ${ }^{15} \mathrm{n}(\vec{x} ; t)$ in an asymptotic series in $\delta$ about an equilibrium function, $\mathbf{n}^{(0)}(\vec{x} ; t)$ (the so-called "ground state" in asymptotic expansion terms),

$$
\mathbf{n}(\vec{x} ; t)=\sum_{j=r}^{\infty} \delta^{j} \mathbf{n}^{(j)}(\vec{x} ; t)
$$

\footnotetext{
${ }^{13}$ The term, "hydrodynamical equations", will be formally defined upon the introduction of sufficient notation.

${ }^{14}$ An example of how differing relationships between $\Delta x$ and $\Delta t$ in standard finite difference schemes correspond to differing sets of consistent equations may be found in [66, p. 177].

${ }^{15}$ In this section $\mathbf{n}=\mathbf{n}\left(\vec{x}_{i} t\right)$ does not denote the occupation numbers as computed by a lattice Boltzmann method; rather, it denotes an equilibrium expansion. Note also that in this section (only) does $\mathbf{n}_{r}^{n}$ denote $\mathbf{n}(\vec{\imath} \Delta x ; n \Delta t)$. Elsewhere $\mathbf{n}_{r}^{n}$ denotes the vector of occupation numbers as computed by a lattice Boltzmann method.
} 
(In the remainder of this section, we use $\mathrm{n}$ and $\mathrm{n}^{(j)}$ to denote $\mathrm{n}(\vec{x} ; t)$ and $\mathrm{n}^{(j)}(\vec{x} ; t)$, respectively.) Equilibrium $\mathrm{n}^{(0)}$ is determined by the equilibrium analysis of the last section.

2. Generate the $(p+1)$-dimensional ${ }^{16}$ Taylor expansion of the advection operator,

$$
\begin{aligned}
\mathcal{A} \mathrm{n}_{\mathfrak{r}}^{n} & =\left[\left(n_{k}\right)_{\Gamma+\varepsilon_{k}}^{n+1}\right] \\
& =\mathrm{n}_{\mathfrak{r}}^{n}+\text { (higher order terms) }
\end{aligned}
$$

about $(\vec{\imath} \Delta x ; m \Delta t)$, the temporal and spatial scales related by

$$
\begin{aligned}
\Delta x & =\delta L \\
\Delta t & =\delta^{k} T
\end{aligned}
$$

in which $L$ and $T$ are the spatial and temporal scale lengths, respectively, and $k$ is an appropriate order for relating the spatial and temporal scales. A usual choice for $k$ in diffusion-related problems is $k=2$, since diffusion occurs on an $\mathcal{O}\left[(\Delta x)^{2}\right]$ time scale. (We use $k=2$ throughout the dissertation.) Different choices may yield different sets of consistent equations, as previously indicated. We substitute the asymptotic series for $\mathbf{n}$ into the Taylor series expansion of the advection operator, then rearrange the resulting expansion to obtain the coefficients of powers of $\delta$.

3. Generate the 1-dimensional Taylor expansion of the collision operator. To do this, first substitute the asymptotic expansion for $\mathbf{n}$ into the collision operator so that

$$
\mathcal{C}(\mathbf{n})=\mathcal{C}\left(\sum_{j=0}^{\infty} \delta^{j} \mathbf{n}^{(j)}\right)
$$

\footnotetext{
${ }^{16}$ There are $p$ spatial dimensions and 1 temporal dimension.
} 
Then define an auxiliary function, $c(\delta)$, by

$$
c(\delta) \equiv \mathcal{C}\left(\sum_{j=0}^{\infty} \delta^{j} \mathbf{n}^{(j)}\right)
$$

for fixed $\mathbf{n}$. Then expand $c(\delta)$ in a Taylor series about $\delta=0$ to get

$$
c(\delta)=c(0)+\delta c^{\prime}(0)+\frac{\delta^{2}}{2 !} c^{\prime \prime}(0)+\cdots+\frac{\delta^{j}}{j !} c^{[j]}(0)+\cdots,
$$

where

$$
\left.c^{[j]}(0) \equiv\left(\frac{d^{j}}{d \delta^{j}} c(\delta)\right)\right|_{\delta=0}
$$

4. Equate the coefficients of like powers of $\delta$ on both sides of the expanded form of the Lattice Boltzmann Equation, Eq. 2.8.

5. The $\mathrm{n}^{(j)}$ are to be determined by equating the coefficients of the Taylor series expansions for each side of Eq. 2.8. This sets up a linear system of equations involving the linearized collision operator of the lattice method (see Definition $2.32)$. Since the linearized collision operator will be seen to be singular, the linear systems either have no solution or an infinite number of solutions. Imposing compatibility, or consistency conditions guarantees that solutions exist at each order in matching the expansions, i.e., the $\mathrm{n}^{(j)}$ coefficients can be determined. Such conditions introduce one degree of freedom (or parameter) at each order for each dimension of the nullspace of the linearized collision operator. These parameters, $\left\{\sigma^{(0)}, \sigma^{(1)}, \sigma^{(2)}, \ldots\right\}$, are determined by matching higher orders in the expansions. ${ }^{17}$ Note that these parameters are vectors having as many elements as the dimension of the nullspace of the linearized collision operator. ${ }^{18}$

\footnotetext{
${ }^{17} \sigma^{(0)}$ is determined by an equilibrium solution.

${ }^{18}$ In the case that the $\operatorname{dim}($ nullspace $(\mathcal{L}))=1$, the $\sigma^{(k)}$ are scalars.
} 


\subsubsection{Equilibrium Expansion}

Suppose the equilibrium solution of a lattice method is given. (Theorem 2.25 may have been used to determine this if the lattice method satisfies semi-detailed balunce.) In any case, the assumption is that we have some equilibrium solution, $\mathfrak{n}^{(0)}$.

Example (L.B.: Equilibrium Solution).

For $L B_{1}$, the equilibrium solution is $\mathbf{n}^{(0)}=\left[\begin{array}{l}u \\ u \\ u \\ u\end{array}\right]$, where $\sigma^{(0)} \equiv u$ is the $\mathcal{O}[1]$

parameter, which we will find satisfies the consistency condition at $\mathcal{O}\left[\delta^{2}\right]$. We applied both the Equilibrium Theorem and the direct method to obtain the form of the equilibrium solutions for $L B_{1}$.

Given an equilibrium, we assume existence of an asymptotic expansion of $n(\vec{x} ; t) \in$ $[0,1]^{d}$ about $\mathbf{n}^{(0)}(\vec{x} ; t) \in[0,1]^{d}$ :

$$
\mathbf{n}(\vec{x} ; t) \equiv \sum_{j=0}^{\infty} \delta^{j} \mathbf{n}^{(j)}(\vec{x} ; t)
$$

where $\delta \ni 0<\delta \ll 1$ is a small dimensionless parameter that is related to the unit spatial and temporal scale lengths, $\Delta x$ and $\Delta t$. This may be recognized as forming the basis of the well-known Chapman-Enskog expansion, which is discussed in $[44,45]$. A couple of points are now in order. The first point regards existence and convergence of the r.h.s. of Eq. 2.14; the second point is about the discrete nature of our Chapman-Enskog expansion. Grad $[44,45]$ notes that the expansion may not converge or even exist, depending upon the initial and boundary conditions. Here, the expansion is assumed to exist, and its validity is discussed in the convergence 
analysis of Section 2.4. In the Chapman-Enskog procedure of $[44,45]$ the expansion, Eq. 2.14, vicwed as a function of $\vec{a}$ and $t$, is substituted into the Boltzmann equation. ()ur expansion is substituted into the Lattice Boltamann Equation, Eq. 2.8, and is thercfore evaluated for only discrete space and time; hence, the term the discrete Chapman-Enskog expansion. The $\mathrm{n}^{(j)} \mathrm{in} \mathrm{Eq} \cdot 2.14$ are determined when applying the discrete Chapman-Enskog procedure. This procedure is the process of matching the advection and collision operator expansions to determine the $\mathbf{n}^{(j)}$ coefficients and the associated consistency conditions.

Depending on the particular lattice method, certain restrictions must hold for existence of the terms of the expansion. This will be apparent when matching the advection and collision operator expansions; the $\mathbf{n}^{(j)}$ in Eq. 2.14 are determined in matching the expansions. Note that $\mathbf{n}^{(j)}$ is a function of the parameters $\left\{\sigma^{(0)}, \sigma^{(1)}, \ldots, \sigma^{(j-1)}\right\}$, which in turn are functions of $\vec{x}$ and $t$, but are evaluated only at the lattice nodes $\vec{\imath}$ and time steps $n$.

\subsubsection{Linearized Collision Operator}

This section defines the linearized collision operator and discusses its eigenvalues and eigenvectors and pseudo-inverse. The relevance of the componentwise vector product for the eigenvectors is also discussed.

The linearized collision operator, $\mathcal{L}$, is to be defined as a linearization of the zeroth order contribution of the collision operator ${ }^{10}$ about a non-trivial equilibrium, $\mathrm{n}^{(0)}$. Our discrete Chapman-Enskog analysis applies to the case where the collision

\footnotetext{
${ }^{19}$ See p. 32 regarding ordering the lattice Boltamann collision operator.
} 
operator can be expressed (for fixed $\mathbf{n}$ ) as

$$
\mathcal{C}(\mathbf{n})=\mathcal{C}^{(0)}(\mathbf{n})+\delta \mathcal{C}^{(1)}(\mathbf{n})
$$

for some $\mathcal{C}^{(0)}(\mathbf{n})$ and $\mathcal{C}^{(1)}(\mathbf{n})$. (In our example method, i.e., $\mathrm{LB}_{1}, \mathcal{C}^{(0)}(\mathbf{n})=0$.) That the collision operator can be expanded to first order in $\delta$ encompasses lattice methods with an advection bias on the order of $\delta$. This, coupled with the Fermi Exclusion Principle leads to methods for nonlinear advection-diffusion systems. (Advection biases are addressed in the analysis for the two example lattice methods in Sections 3.2 and 3.3.) In our treatment, we assume that the highest orcier in the collision operator is $\mathcal{O}[\delta]$. The linearized collision operator and its pseudo-inverse, $\mathcal{L}^{+}$, are used in the the discrete Chapman-Enskog expansion.

Definition 2.32 (Linearized Collision Operator). The linearized collision operator, $\mathcal{L}$, of a lattice method with collision operator, $\mathcal{C}$, operating on the mean occupation numbers, $\mathbf{n}$, is defined by the $d \times d$ matrix

$$
\mathcal{L} \equiv\left[\mathcal{L}_{k, l}\right]_{k, l=0}^{d-1}
$$

where

$$
\left.\mathcal{L}_{k, l} \equiv \frac{\partial}{\partial n_{l}} \mathcal{C}_{k}^{(0)}(\mathbf{n})\right|_{\mathbf{n}=\mathbf{n}^{(0)}}
$$

and $\mathrm{n}^{(0)}$ is an equilibrium solution of the lattice method.

The linearized collision operator is a linearization of the zeroth order, i.e., $\mathcal{O}[1]$, contribution of the collision operator about a non-trivial equilibrium. Not: that

$$
\mathcal{L}=\mathcal{D} \mathcal{C}^{(0)}\left(\mathbf{n}^{(0)}\right)=\frac{\partial}{\partial \mathbf{n}} \mathcal{C}\left(\mathbf{n}^{(0)}\right)=\left.\frac{\partial}{\partial \mathbf{n}} \mathcal{C}(\mathbf{n})\right|_{\mathbf{n}=\mathbf{n}^{(0)}}
$$


For all the lattice methods studied herein, $\mathcal{L}$, is a symmetric, nonpositive definite circulant. ${ }^{20}$ This is because for all of our methods: (1) they have rotation and reflection ${ }^{21}$ invariances (these are isomorphic to the permutation and reflection isometries in the the isometry group $\mathfrak{G}$ globally preserving the set of velocity vectors on our two-dimensional lattice (see Definition 2.11)), (2) the linearization of the collision operator is about an isotropic equilibrium, i.e., it is invariant under the isometries of the lattice (see Definition 2.7), and (3) they satisfy semi-detailed balance ${ }^{22}$ (see Definition 2.16). Reasons (1) and (2) guarantee that $\mathcal{L}$ is a symmetric circulant; the addition of (3), which implies the existence of an entropy through local and global H-theorems (see [37]), guarantees nonpositive definiteness.

\section{Example ( $L B_{1}$ : Linearized Collision Operator).}

First, note that for $L B_{1}, \mathcal{C}\left(\mathbf{n}^{(0)}\right)=\mathcal{C}^{(0)}\left(\mathbf{n}^{(0)}\right)$, since it contains only $\mathcal{O}[1]$ terms, i.e., all higher order terms $\left(\mathcal{C}^{(k)}\left(\mathbf{n}^{(0)}\right)\right.$, for $\left.k \geq 1\right)$ are zero. Henceforth we write $\mathcal{C}(\mathbf{n})$ for $\mathcal{C}^{(0)}(\mathbf{n})$ in this example method. Then the linearized collision operator for $\mathrm{LB}_{1}$ is given below with $k \in\{0,1,2,3\}$, ${ }^{23}$

$$
\begin{aligned}
& \left.\frac{\partial}{\partial n_{k}} \mathcal{C}_{k}(\mathbf{n})\right|_{\mathbf{n}=\mathbf{n}(0)} \\
& \quad=\left(-\bar{n} \overline{k+1} n_{k+2} n_{k+3}-n_{k+1} \overline{n_{k+2}} n_{k+3}-n_{k+1} n_{k+2} \overline{n_{k+3}}\right.
\end{aligned}
$$

${ }^{20} \mathrm{~A} d \times d$ circulant (see for example, $[3, \mathrm{pp}, 242-4]$ ) is a $d \times d$ matrix of the form,

$$
\mathcal{L}=\left[\begin{array}{llll}
c_{0} & c_{1} & \cdots & c_{d-1} \\
c_{d-1} & c_{0} & \cdots & c_{d-2} \\
\vdots & & \ddots & \vdots \\
c_{1} & c_{2} & \cdots & c_{0}
\end{array}\right]
$$

\footnotetext{
${ }^{21}$ Reflection invariance is a symmetry about the axes spanned by each velocity vector $\vec{e}_{k}$. (It is analogous to parity-invariance in [37].) This definition suffices for two-dimensional problems on both the hexagonal and square lattices.

${ }^{22}$ Weaker assumptions are being considered (cf. quasi-detailed balance, Definition 2.17) [54].

${ }^{23}$ Recall that the indices are evaluated modulo $d=4$.
} 


$$
\begin{aligned}
& \left.-\overline{n_{k+1}} \pi_{k+2} n_{k+3}-\pi \pi_{k+1} n_{k+2} \overline{n_{k+3}}-n_{k+1} \overline{n_{k+2}} \overline{n_{k+3}}\right)\left.\right|_{\mathbf{n}=\mathbf{n}^{(0)}} \\
& =-\bar{u} u u-u \bar{u} u-u u \bar{u}-\bar{u} \bar{u} u-u \bar{u} u \\
& =-3 u \pi=-3 u(1-u), \\
& \left.\frac{\partial}{\partial n_{k+1}} \mathcal{C}_{k}(\mathbf{n})\right|_{\mathbf{n}=\mathbf{n}^{(0)}} \\
& =\left(-\overline{n_{k}} n_{k+2} n_{k+3}+\overline{n_{k}} \overline{n_{k+2}} n_{k+3}+\overline{n_{k}} n_{k+2} \overline{n_{k+3}}\right. \\
& \left.+n_{k} \overline{n_{k+2}} n_{k+3}+n_{k} n_{k+2} \overline{n_{k+3}}-n_{k} \overline{n_{k+2}} \overline{n_{k+3}}\right)\left.\right|_{\mathbf{n}=\mathbf{n}^{(0)}} \\
& =u \bar{u}=u(1-u) \text {, } \\
& \left.\frac{\partial}{\partial n_{k+2}} \mathcal{C}_{k}(\mathbf{n})\right|_{\mathbf{n}=\mathbf{n}^{(0)}} \\
& =\left(+\overline{n_{k}} \overline{n_{k+1}} n_{k+3}-\overline{n_{k}} n_{k+1} n_{k+3}+\overline{n_{k}} n_{k+1} \overline{n_{k+3}}\right. \\
& \left.+n_{k} \overline{n_{k+1}} n_{k+3}-n_{k} \bar{n}_{k+1} \overline{n_{k+3}}+n_{k} n_{k+1} \overline{n_{k+3}}\right)\left.\right|_{\mathbf{n}=\mathbf{n}(0)} \\
& =u \bar{u}=u(1-u) \text {, } \\
& \left.\frac{\partial}{\partial n_{k+3}} \mathcal{C}_{k}(\mathbf{n})\right|_{\mathbf{n}=\mathbf{n}^{(0)}} \\
& =\left(+\overline{n_{k}} \overline{n_{k+1}} n_{k+2}+\overline{n_{k}} n_{k+1} \overline{n_{k+2}}-\overline{n_{k}} n_{k+1} n_{k+2}\right. \\
& \left.-n_{k} \overline{n_{k+1}} \overline{n_{k+2}}+n_{k} \overline{n_{i+1}} n_{k+2}+n_{k} n_{k+1} \overline{n_{k+2}}\right)\left.\right|_{\mathbf{n}=\mathbf{n}^{(0)}} \\
& =u \bar{u}=u(1-u) \text {. }
\end{aligned}
$$

Hence, the linearized collision operator may be written,

$$
\mathcal{L}=-\frac{\lambda}{4}\left[\begin{array}{rrrr}
-3 & 1 & 1 & 1 \\
1 & -3 & 1 & 1 \\
1 & 1 & -3 & 1 \\
1 & 1 & 1 & -3
\end{array}\right]
$$

where $\lambda=-4 u \bar{u}=-4 u(1-u)$. Note that indeed $\mathcal{L}$ is a symmetric, nonpositive definite circulant. 


\section{Eigenvalues and Eigenvectors}

The eigenvectors and eigenvalues of the linearized collision operator are important in the discrete Chapman-Enskog analysis. They are also important in determining the conserved quantities in a lattice Boltzmann method [37]. Note that they may be easily found for circulant linearized collision operators (see, for example, [3, pp. 2424]). A symbolic mathematical manipulation tool, such as Mathematica, can be used to determine the eigenpairs. Given that the linearized collision operator is symmetric it possesses a complete set of real orthogonal eigenvectors.

Definition 2.33 (Eigenmatrix). Let $\left\{\mathbf{q}_{0}, \mathbf{q}_{1}, \ldots, \mathbf{q}_{d-1}\right\}$ be a set of real orthogonal (column) eigenvectors of $\mathcal{L}$ with corresponding real eigenvalues $\left\{\lambda_{0}, \lambda_{1}, \ldots, \lambda_{d-1}\right\}$. Then the eigenmatrix, $\mathbf{Q}$, is defined by

$$
\mathbf{Q} \equiv\left[\mathbf{q}_{0}, \mathbf{q}_{1}, \ldots, \mathbf{q}_{d-1}\right]
$$

The normalized eigenmatrix, $\hat{\mathbf{Q}}$, is defined to be

$$
\hat{\mathbf{Q}} \equiv\left[\hat{\mathbf{q}}_{0}, \hat{\mathbf{q}}_{1}, \ldots, \hat{\mathbf{q}}_{d-1}\right]
$$

where $\hat{\mathbf{q}}_{k} \equiv \mathbf{q}_{k} /\left\|\mathbf{q}_{k}\right\|_{2}$.

\section{Example ( $L B_{1}$ : Eigenpairs of the Linearized Collision Operator).}

The set of unnormalized eigenpairs (eigenvalue, eigenvector) of our example's linearized collision operator, $\mathcal{L}$, is

$$
\left\{\left(\lambda_{k}, \mathbf{q}_{k}\right) \mid k \in\{0,1,2,3\}\right\}
$$

where the eigenvalues are given by

$$
\text { eigenvalues }(\mathcal{L})=\left(\lambda_{0}, \lambda_{1}, \lambda_{2}, \lambda_{3}\right)=(0,-4 u \bar{u},-4 u \bar{u},-4 u \bar{u}),
$$


and the eigenvectors are given as the eigenmatrix,

$$
\mathbf{Q}=\left[\begin{array}{llll}
\mathbf{q}_{0}, & \mathbf{q}_{1}, & \mathbf{q}_{2}, & \mathbf{q}_{3}
\end{array}\right]=\left[\begin{array}{rrrr}
1 & 1 & 0 & 1 \\
1 & 0 & 1 & -1 \\
1 & -1 & 0 & 1 \\
1 & 0 & -1 & -1
\end{array}\right],
$$

in which the $\mathbf{q}_{k}$ are column vectors.

Recall that the nullspace of a matrix is the span of the eigenvectors corresponding to the zero eigenvalue.

\section{Example ( $L B_{1}$ : Nullspace of Linearized Collision Operator).}

In our example, the nullspace of the linearized collision operator is given by

$$
\operatorname{nullspace}(\mathcal{L})=\operatorname{span}\left(\mathbf{q}_{0}\right)
$$

The linearized collision operator may be expressed in terms of its eigenpairs. Let $\Lambda \equiv \operatorname{diag}\left(\lambda_{k}\right)_{k=0}^{d-1}$. Then

$$
\mathcal{L}=\hat{\mathbf{Q}}^{T} \Lambda \hat{\mathbf{Q}}=\sum_{k=0}^{d-1} \lambda_{k} \frac{\mathbf{q}_{k} \mathbf{q}_{k}^{T}}{\mathbf{q}_{k} \cdot \mathbf{q}_{k}} .
$$

Since the linearized collision operator has a nonempty nullspace it has no inverse; however, we can define a pseudo-inverse, $\mathcal{L}^{+}$, as follows:

Definition 2.34 (Pseudo-Inverse). Let linearized collision operator, $\mathcal{L} \in$ $\mathcal{R}^{d}$, be symmetric with a complete set of real orthogonal eigenvectors $\left\{\mathbf{q}_{0}, \mathbf{q}_{1}, \ldots, \mathbf{q}_{d-1}\right\}$ and respective eigenvalues $\left\{\lambda_{0}, \lambda_{1}, \ldots, \lambda_{d-1}\right\}$. Let $\hat{\mathbf{Q}}$ be the normalized eigenmatrix and let $\Lambda=\operatorname{diag}\left(\lambda_{k}\right)_{k=0}^{d-1}$. Also let $\Lambda^{+} \equiv \operatorname{diag}\left(f\left(\lambda_{k}\right)\right)_{k=0}^{d-1}$, where

$$
f(\lambda) \equiv \begin{cases}1 / \lambda, & \text { if } \lambda \neq 0 \\ 0, & \text { other wise. }\end{cases}
$$


Then the pseudo-inverse of $\mathcal{L}$, denoted $\mathcal{L}^{+}$, is defined by

$$
\mathcal{L}^{+} \equiv \hat{\mathbf{Q}}^{T} \Lambda^{+} \hat{\mathbf{Q}}=\sum_{k \ni \lambda_{k} \neq 0} \frac{1}{\lambda_{k}} \frac{\mathbf{q}_{k} \mathbf{q}_{k}^{T}}{\mathbf{q}_{k} \cdot \mathbf{q}_{k}}
$$

This is consistent to the definition given in Golub and Van Loan [41, pp. 138-9].

Example ( $L B_{1}$ : Pseudo-Inverse of Linearized Collision Operator).

From Eq. 2.15 and Eq. 2.16 we have

$$
\begin{aligned}
\mathcal{L} & =\sum_{k=0}^{3} \lambda_{k} \frac{\mathbf{q}_{k} \mathbf{q}_{k}^{T}}{\mathbf{q}_{k}^{T} \mathbf{q}_{k}}=\lambda\left[\frac{1}{2}\left(\mathbf{q}_{1} \mathbf{q}_{1}^{T}+\mathbf{q}_{2} \mathbf{q}_{2}^{T}\right)+\frac{1}{4} \mathbf{q}_{3} \mathbf{q}_{3}^{T}\right] \\
\mathcal{L}^{+} & =\sum_{k \ni \lambda_{k} \neq 0} \frac{1}{\lambda_{k}} \frac{\mathbf{q}_{k} \mathbf{q}_{k}^{T}}{\mathbf{q}_{k}^{T} \mathbf{q}_{k}}=\frac{1}{\lambda}\left[\frac{1}{2}\left(\mathbf{q}_{1} \mathbf{q}_{1}^{T}+\mathbf{q}_{2} \mathbf{q}_{2}^{T}\right)+\frac{1}{4} \mathbf{q}_{3} \mathbf{q}_{3}^{T}\right],
\end{aligned}
$$

where $\lambda \equiv-4 u \bar{u}=-4 u(1-u)$.

\section{Componentwise Eigenvector Product}

The componentwise vector product ( $\star$ operator) between eigenvectors of the linearized collision operator will be employed to simplify calculations in carrying out the discrete Chapman-Enskog procedure. Recalling the definition of the $\star$ componentwise product operator in Section 2.1.1, we see that for two eigenvectors, $\mathbf{q}_{i}$ and $\mathbf{q}_{j}$, their componentwise vector product is

$$
\mathbf{q}_{i} \star \mathbf{q}_{j}=\left[\mathbf{q}_{i, k} \mathbf{q}_{j, k}\right]_{k=0}^{d-1}
$$

in which $\mathrm{q}_{i, k}$ denotes the $k$ th element of the $i$ th eigenvector. Also recall our convention that the $\star$ operator has the same precedence in expressions as scalar multiplication. For example, * operations would be evaluated before vector additions. We will also refer to the $\star$ operator as the componentuise eigenvector product when applicable. 
Table 2.4: $\mathrm{LB}_{1}$ Componentwise Eigenvector Products.

\begin{tabular}{||c||c|c|c|c||}
\hline \hline$\star$ & $\mathrm{q}_{0}$ & $\mathrm{q}_{1}$ & $\mathrm{q}_{2}$ & $\mathrm{q}_{3}$ \\
\hline \hline $\mathrm{q}_{0}$ & $\mathrm{q}_{0}$ & $\mathrm{q}_{1}$ & $\mathrm{q}_{2}$ & $\mathrm{q}_{3}$ \\
\hline $\mathrm{q}_{1}$ & $\mathrm{q}_{1}$ & $\frac{1}{2}\left(\mathrm{q}_{0}+\mathrm{q}_{3}\right)$ & 0 & $\mathrm{q}_{1}$ \\
\hline $\mathrm{q}_{2}$ & $\mathrm{q}_{2}$ & 0 & $\frac{1}{2}\left(\mathrm{q}_{0}-\mathrm{q}_{3}\right)$ & $-\mathrm{q}_{2}$ \\
\hline $\mathrm{q}_{3}$ & $\mathrm{q}_{3}$ & $\mathrm{q}_{1}$ & $-\mathrm{q}_{2}$ & $\mathrm{q}_{0}$ \\
\hline \hline
\end{tabular}

In the forthcoming discrete Chapman-Enskog analysis, using componentwise products will simplify calculations. The simplification is in expressing the product's result as a linear combination of the eigenvectors. Then dot products of such an expression with an eigenvector becomes trivial (in the light that the dot product of orthogonal eigenvectors is zero).

The componentwise product of two eigenvectors can be expressed as a linear combination of the eigenvectors of the linearized collision operator, for $\mathrm{q}_{k} \star \mathrm{q}_{l} \in$ $\operatorname{span}\left(\mathbf{q}_{k}\right)_{k=0}^{d-1}$. Given the eigenvectors of $\mathcal{L}$ we can tabulate all possible componentwise products. Determining the discrete Chapman-Enskog expansion makes extensive use of such a table so that vectors, $v \in \mathcal{R}^{d}$, can be readily expressed in terms of the basis $\left\{\mathbf{q}_{0}, q_{1}, \ldots, \mathbf{q}_{d-1}\right\}$.

Example ( $L B_{1}$ : Componentwise Eigenvector Products).

The componentwise products of the eigenvectors of the linearized collision operator of $L B_{1}$ are tabulated in Table 2.4. Some exainples follow to show how the entries in the table are determined. 
1. Evaluate $q_{1} \star q_{2}$ :

$$
\mathbf{q}_{1} \star \mathbf{q}_{2}=\left[\begin{array}{c}
1 \\
0 \\
-1 \\
0
\end{array}\right] \star\left[\begin{array}{c}
0 \\
1 \\
0 \\
-1
\end{array}\right]=\left[\begin{array}{c}
(1)(0) \\
(0)(1) \\
(-1)(0) \\
(0)(-1)
\end{array}\right]=\left[\begin{array}{l}
0 \\
0 \\
0 \\
0
\end{array}\right]=0
$$

2. Evaluate $\mathbf{q}_{2} \star \mathbf{q}_{2}$ :

$$
\begin{aligned}
\mathbf{q}_{2} \star \mathbf{q}_{2} & =\left[\begin{array}{c}
0 \\
1 \\
0 \\
-1
\end{array}\right] \star\left[\begin{array}{c}
0 \\
1 \\
0 \\
-1
\end{array}\right]=\left[\begin{array}{c}
(0)(0) \\
(1)(1) \\
(0)(0) \\
(-1)(-1)
\end{array}\right]=\left[\begin{array}{l}
0 \\
1 \\
0 \\
1
\end{array}\right] \\
& =\frac{1}{2}\left(\mathbf{q}_{0}-\mathbf{q}_{3}\right) .
\end{aligned}
$$

3. Evaluate $q_{2} \star q_{3}$ :

$$
\begin{aligned}
\mathbf{q}_{2} \star \mathbf{q}_{3} & =\left[\begin{array}{c}
0 \\
1 \\
0 \\
-1
\end{array}\right] \star\left[\begin{array}{c}
1 \\
-1 \\
1 \\
-1
\end{array}\right]=\left[\begin{array}{c}
(0)(1) \\
(1)(-1) \\
(0)(1) \\
(-1)(-1)
\end{array}\right]=\left[\begin{array}{c}
0 \\
-1 \\
0 \\
1
\end{array}\right] \\
& =-\left[\begin{array}{c}
0 \\
1 \\
0 \\
-1
\end{array}\right] \\
& =-\mathbf{q}_{2} .
\end{aligned}
$$


Using Componentwise Eigenvector Products

Given a table like Table 2.4 for all componentwise eigenvector products, we will often have to calculate an expression like

$$
S=q_{1} \cdot\left\{q_{3} \star\left[\begin{array}{c}
+\frac{\partial}{\partial x} u \\
+\frac{\partial}{\partial y} u \\
-\frac{\partial}{\partial x} u \\
-\frac{\partial}{\partial y} u
\end{array}\right]\right\} \text { (cf. the example on p. 69) }
$$

To do the calculation, first we write the vector expression

$$
\left[\begin{array}{l}
+\frac{\partial}{\partial x} u \\
+\frac{\partial}{\partial y} u \\
-\frac{\partial}{\partial x} u \\
-\frac{\partial}{\partial y} u
\end{array}\right]
$$

as a linear combination of eigenvectors of $\mathcal{L}$. Then we use the componentwise eigenvector product table (Table 2.4) to simplify the expression, after which we complete the dot product. In calculating the dot product, we use orthogonality of distinct eigenvectors. We find

$$
\begin{aligned}
S & =\mathbf{q}_{1} \cdot\left\{\mathbf{q}_{3} \star\left(\mathbf{q}_{1} \frac{\partial}{\partial x} u+\mathbf{q}_{2} \frac{\partial}{\partial y} u\right)\right\} \\
& =\mathbf{q}_{1} \cdot\left(\left(\mathbf{q}_{3} \star \mathbf{q}_{1}\right) \frac{\partial}{\partial x} u+\left(\mathbf{q}_{3} \star \mathbf{q}_{2}\right) \frac{\partial}{\partial y} u\right) \\
& =\mathbf{q}_{1} \cdot\left(\mathbf{q}_{1} \frac{\partial}{\partial x} u-\mathbf{q}_{2} \frac{\partial}{\partial y} u\right) \\
& =\mathbf{q}_{1} \cdot \mathbf{q}_{1} \frac{\partial}{\partial x} u-\mathbf{q}_{1} \cdot \mathbf{q}_{2} \frac{\partial}{\partial y} u \\
& =2 \frac{\partial}{\partial x} u-0=2 \frac{\partial}{\partial x} u .
\end{aligned}
$$


(alculations involving complicated vector expressions and dot products can become much simpler with the $\star$ componentwise product.

\subsubsection{Collision Operator Expansion}

For fixed $n$ (and fixed $\left\{\mathbf{n}^{(i)} \mid j \geq 0\right\}$ ), define $c: \mathcal{R} \rightarrow[0,1]^{d}$ by

$$
\begin{aligned}
c(\delta) & \equiv \mathcal{C}\left(\mathbf{n}^{(0)}+\delta \mathbf{n}^{(1)}+\delta^{2} \mathbf{n}^{(2)}+\cdots\right) \\
& =\mathcal{C}^{(0)}\left(\mathbf{n}^{(0)}+\delta \mathbf{n}^{(1)}+\delta^{2} \mathbf{n}^{(2)}+\cdots\right)+\delta \mathcal{C}^{(1)}\left(\mathbf{n}^{(0)}+\delta \mathbf{n}^{(1)}+\delta^{2} \mathbf{n}^{(2)}+\cdots\right)
\end{aligned}
$$

Note that $c \in C^{\infty}\left(\mathcal{R},[0,1]^{d}\right)$ because $\mathcal{C}(\mathbf{n})$ is a polynomial-type function in the $n_{k}$ and since $\mathrm{n}$ is polynomial in $\delta$. Then by Taylor's theorem ${ }^{24}$,

$$
c(\delta)=c(0)+c^{\prime}(0)+\frac{\delta^{2}}{2 !} c^{\prime \prime}(0)+\frac{\delta^{3}}{3 !} c^{\prime \prime \prime}(0)+\frac{\delta^{4}}{4 !} c^{[i v]}(0)+\cdots,
$$

where

$$
\left.c^{[j]}(0) \equiv\left(\frac{d^{j}}{d \delta^{j}} c(\delta)\right)\right|_{\delta=0} .
$$

Eq. 2.17 is the expansion for the collision operator, which will become apparent through application of the chain rule.

To determine the coefficients in Eq. 2.17, we look at the derivatives of $c(\delta)$ evaluated at $\delta=0$. First, note that

$$
\begin{aligned}
\mathbf{n}^{\prime} & \equiv \frac{d}{d \delta} \mathbf{n}=\frac{d}{d \delta}\left(\mathbf{n}^{(1)}+\delta \mathbf{n}^{(1)}+\delta^{2} \mathbf{n}^{(2)}+\delta^{3} \mathbf{n}^{(3)}+\cdots\right) \\
& =\mathbf{n}^{(1)}+2 \delta \mathbf{n}^{(2)}+3 \delta^{2} \mathbf{n}^{(3)}+\cdots, \\
\mathbf{n}^{\prime \prime} & =\frac{d^{2}}{d \delta^{2}} \mathbf{n}=\frac{d}{d \delta} \mathbf{n}^{\prime} \\
& =2 \mathbf{n}^{(2)}+6 \delta \mathbf{n}^{(3)}+12 \delta^{2} \mathbf{n}^{(4)}+\cdots,
\end{aligned}
$$

\footnotetext{
${ }^{24}$ For a statement (and a proof) of 'Taylor's Theorem see, for example, [55, Section 6.8 ('Theorem 10)].
} 


$$
\begin{aligned}
\mathbf{n}^{[j]} & =\frac{d^{j}}{d \delta^{j}} \mathbf{n}=\frac{d^{j}}{d \delta^{j}}\left(\mathbf{n}^{(0)}+\delta \mathbf{n}^{(1)}+\delta^{2} \mathbf{n}^{(2)}+\delta^{3} \mathbf{n}^{(3)}+\cdots\right) \\
& =j \mid \mathbf{n}^{(j)}+\frac{(j+1) !}{2 !} \delta \mathbf{n}^{(j+1)}+\frac{(j+2) !}{3 !} \delta^{2} \mathbf{n}^{(j+2)}+\cdots
\end{aligned}
$$

and in general

$$
\left.\mathbf{n}^{[j]}\right|_{\delta=0}=\left.\frac{d^{j}}{d \delta^{j}} \mathbf{n}\right|_{\delta=0}=j \mid \mathbf{n}^{(j)}
$$

Also note that in determining the coefficients we will apply the the chain and product rules for differentiation, e.g.,

$$
\frac{d}{d \delta} \mathcal{C}^{(0)}(\mathbf{n})=\frac{\partial}{\partial \mathbf{n}} \mathcal{C}^{(0)}(\mathbf{n}) \cdot \frac{d}{d \delta} \mathbf{n}=\mathcal{D} \mathcal{C}^{(0)}(\mathbf{n}) \cdot \mathbf{n}^{\prime},
$$

and

$$
\begin{aligned}
& \frac{d}{d \delta} \mathcal{D}^{2} \mathcal{C}^{(0)}(\mathbf{n}) \cdot \mathbf{n}^{\prime} \mathbf{n}^{\prime \prime} \\
& \quad=\mathcal{D}^{2} \mathcal{C}^{(0)}(\mathbf{n}) \cdot \frac{d}{d \delta}\left(\mathbf{n}^{\prime} \mathbf{n}^{\prime \prime}\right)+\mathcal{D}^{3} \mathcal{C}^{(0)}(\mathbf{n}) \cdot \mathbf{n}^{\prime} \mathbf{n}^{\prime} \mathbf{n}^{\prime \prime} \\
& =\mathcal{D}^{2} \mathcal{C}^{(0)}(\mathbf{n}) \cdot\left(\mathbf{n}^{\prime} \mathbf{n}^{\prime \prime \prime}+\mathbf{n}^{\prime \prime} \mathbf{n}^{\prime \prime}\right)+\mathcal{D}^{3} \mathcal{C}^{(0)}(\mathbf{n}) \cdot \mathbf{n}^{\prime} \mathbf{n}^{\prime} \mathbf{n}^{\prime \prime} \\
& \quad=\mathcal{D}^{2} \mathcal{C}^{(0)}(\mathbf{n}) \cdot \mathbf{n}^{\prime} \mathbf{n}^{\prime \prime \prime}+\mathcal{D}^{2} \mathcal{C}^{(0)}(\mathbf{n}) \cdot \mathbf{n}^{\prime \prime} \mathbf{n}^{\prime \prime}+\mathcal{D}^{3} \mathcal{C}^{(0)}(\mathbf{n}) \cdot \mathbf{n}^{\prime} \mathbf{n}^{\prime} \mathbf{n}^{\prime \prime}
\end{aligned}
$$

The coefficients in Eq. 2.17 are found at each order as follows:

$\mathcal{O}[1]:$

$$
\begin{aligned}
c(\delta) & =\mathcal{C}^{(0)}(\mathbf{n})+\delta \mathcal{C}^{(1)}(\mathbf{n}) \\
\Rightarrow c(0) & =\mathcal{C}^{(0)}\left(\mathbf{n}^{(0)}\right) .
\end{aligned}
$$

$\mathcal{O}[\delta]:$

$$
\begin{aligned}
c^{\prime}(\delta) & =\mathcal{D} \mathcal{C}^{(0)}(\mathbf{n}) \cdot \mathbf{n}^{\prime}+\mathcal{C}^{(1)}(\mathbf{n})+\delta \mathcal{D C} \mathcal{C}^{(1)}(\mathbf{n}) \cdot \mathbf{n}^{\prime} \\
\Rightarrow c^{\prime}(0) & =\mathcal{D} \mathcal{C}^{(0)}\left(\mathbf{n}^{(0)}\right) \cdot \mathbf{n}^{(1)}+\mathcal{C}^{(1)}\left(\mathbf{n}^{(0)}\right)
\end{aligned}
$$


(') $\left[6^{2}\right]:$

$$
\begin{aligned}
c^{\prime \prime}(\delta) & =\frac{d}{d \delta}\left(c^{\prime}(\delta)\right) \\
& =\mathcal{D C C}^{(0)}(\mathbf{n}) \cdot \mathbf{n}^{\prime \prime}+\mathcal{D}^{2} \mathcal{C}^{(0)}(\mathbf{n}) \cdot \mathbf{n}^{\prime} \mathbf{n}^{\prime} \\
& +2 \mathcal{D} \mathcal{C}^{(1)}(\mathbf{n}) \cdot \mathbf{n}^{\prime}+\delta\left[\mathcal{D}^{2} \mathcal{C}^{(1)}(\mathbf{n}) \cdot \mathbf{n}^{\prime} \mathbf{n}^{\prime}+\mathcal{D} \mathcal{C}^{(1)}(\mathbf{n}) \cdot \mathbf{n}^{\prime \prime}\right] \\
\Rightarrow c^{\prime \prime}(0) & =2 \mathcal{D} \mathcal{C}^{(0)}\left(\mathbf{n}^{(0)}\right) \cdot \mathbf{n}^{(2)}+\mathcal{D}^{2} \mathcal{C}^{(0)}\left(\mathbf{n}^{(0)}\right) \cdot \mathbf{n}^{(1)} \mathbf{n}^{(1)}+2 \mathcal{D} \mathcal{C}^{(1)}\left(\mathbf{n}^{(0)}\right) \cdot \mathbf{n}^{(1)} .
\end{aligned}
$$

$\mathcal{O}\left[\delta^{3}\right]:$

$$
\begin{aligned}
c^{\prime \prime \prime}(\delta) & =\frac{d}{d \delta}\left(c^{\prime \prime}(\delta)\right) \\
& =\mathcal{D}^{3} \mathcal{C}^{(0)}(\mathbf{n}) \cdot \mathbf{n}^{\prime} \mathbf{n}^{\prime} \mathbf{n}^{\prime}+3 \mathcal{D}^{2} \mathcal{C}^{(0)}(\mathbf{n}) \cdot \mathbf{n}^{\prime} \mathbf{n}^{\prime \prime}+\mathcal{D C} \mathcal{C}^{(0)}(\mathbf{n}) \cdot \mathbf{n}^{\prime \prime \prime} \\
& +3 \mathcal{D}^{2} \mathcal{C}^{(1)}(\mathbf{n}) \cdot \mathbf{n}^{\prime} \mathbf{n}^{\prime}+3 \mathcal{D} \mathcal{C}^{(1)}(\mathbf{n}) \cdot \mathbf{n}^{\prime \prime} \\
& +\delta\left[\mathcal{D}^{3} \mathcal{C}^{(1)}(\mathbf{n}) \cdot \mathbf{n}^{\prime} \mathbf{n}^{\prime} \mathbf{n}^{\prime}+3 \mathcal{D}^{2} \mathcal{C}^{(1)}(\mathbf{n}) \cdot \mathbf{n}^{\prime} \mathbf{n}^{\prime \prime}+\mathcal{D} \mathcal{C}^{(1)}(\mathbf{n}) \cdot \mathbf{n}^{\prime \prime \prime}\right] \\
\Rightarrow c^{\prime \prime \prime}(0) & =\mathcal{D}^{3} \mathcal{C}^{(0)}\left(\mathbf{n}^{(0)}\right) \cdot \mathbf{n}^{(1)} \mathbf{n}^{(1)} \mathbf{n}^{(1)}+6 \mathcal{D}^{2} \mathcal{C}^{(0)}\left(\mathbf{n}^{(0)}\right) \cdot \mathbf{n}^{(1)} \mathbf{n}^{(2)} \\
& +6 \mathcal{D} \mathcal{C}^{(0)}\left(\mathbf{n}^{(0)}\right) \cdot \mathbf{n}^{(3)}+3 \mathcal{D}^{2} \mathcal{C}^{(1)}\left(\mathbf{n}^{(0)}\right) \cdot \mathbf{n}^{(1)} \mathbf{n}^{(1)} \\
& +6 \mathcal{D C} \mathcal{C}^{(1)}\left(\mathbf{n}^{(0)}\right) \cdot \mathbf{n}^{(2)}
\end{aligned}
$$

$\mathcal{O}\left[\delta^{4}\right]:$

$$
\begin{aligned}
c^{(i v)}(\delta) & =\frac{d}{d \delta}\left(c^{\prime \prime \prime}(\delta)\right) \\
& =\mathcal{D}^{4} \mathcal{C}^{(0)}(\mathbf{n}) \cdot \mathbf{n}^{\prime} \mathbf{n}^{\prime} \mathbf{n}^{\prime} \mathbf{n}^{\prime}+6 \mathcal{D}^{3} \mathcal{C}^{(0)}(\mathbf{n}) \cdot \mathbf{n}^{\prime} \mathbf{n}^{\prime} \mathbf{n}^{\prime \prime}+4 \mathcal{D}^{2} \mathcal{C}^{(0)}(\mathbf{n}) \cdot \mathbf{n}^{\prime} \mathbf{n}^{\prime \prime \prime} \\
& +3 \mathcal{D}^{2} \mathcal{C}^{(0)}(\mathbf{n}) \cdot \mathbf{n}^{\prime \prime} \mathbf{n}^{\prime \prime}+\mathcal{D} \mathcal{C}^{(0)}(\mathbf{n}) \cdot \mathbf{n}^{\prime \prime \prime \prime} \\
& +4 \mathcal{D}^{3} \mathcal{C}^{(1)}(\mathbf{n}) \cdot \mathbf{n}^{\prime} \mathbf{n}^{\prime} \mathbf{n}^{\prime}+12 \mathcal{D}^{2} \mathcal{C}^{(1)}(\mathbf{n}) \cdot \mathbf{n}^{\prime} \mathbf{n}^{\prime \prime}+4 \mathcal{D} \mathcal{C}^{(1)}(\mathbf{n}) \cdot \mathbf{n}^{\prime \prime \prime} \\
& +(\mathcal{O}[\delta] \operatorname{terms}) \\
\Rightarrow c^{[i v]}(0) & =\mathcal{D}^{4} \mathcal{C}^{(0)}\left(\mathbf{n}^{(0)}\right) \cdot \mathbf{n}^{(1)} \mathbf{n}^{(1)} \mathbf{n}^{(1)} \mathbf{n}^{(1)}+12 \mathcal{D}^{3} \mathcal{C}^{(0)}\left(\mathbf{n}^{(0)}\right) \cdot \mathbf{n}^{(1)} \mathbf{n}^{(1)} \mathbf{n}^{(2)} \\
& +24 \mathcal{D}^{2} \mathcal{C}^{(0)}\left(\mathbf{n}^{(0)}\right) \cdot \mathbf{n}^{(1)} \mathbf{n}^{(3)}+12 \mathcal{D}^{2} \mathcal{C}^{(1)}\left(\mathbf{n}^{(1)}\right) \cdot \mathbf{n}^{(2)} \mathbf{n}^{(2)}
\end{aligned}
$$




$$
\begin{aligned}
& +24 \mathcal{D} \mathcal{C}^{(0)}\left(n^{(0)}\right) \cdot n^{(4)}+4 \mathcal{D}^{3} \mathcal{C}^{(1)}\left(n^{(0)}\right) \cdot n^{(1)} n^{(1)} n^{(1)} \\
& +24 \mathcal{D}^{2} \mathcal{C}^{(1)}\left(n^{(0)}\right) \cdot n^{(1)} n^{(2)}+24 \mathcal{D C} \mathcal{C}^{(1)}\left(n^{(0)}\right) \cdot n^{(3)}
\end{aligned}
$$

Finally, substituting the $c^{(j)}(0)$ into Eq. 2.17 and using the notation $\mathcal{L}=\mathcal{D} \mathcal{C}^{(0)}\left(\mathbf{n}^{(0)}\right)$ (see Definition 2.32), we obtain the Chapman-Enskog-Taylor asymptotic expansion of the collision operator about equilibrium, $\mathbf{n}^{(0)}$,

$$
\begin{aligned}
\mathcal{C}(\mathbf{n})= & c(\delta) \\
= & \mathcal{C}^{(0)}\left(\mathbf{n}^{(0)}\right) \\
+ & \delta\left[\mathcal{L} \cdot \mathbf{n}^{(1)}+\mathcal{C}^{(1)}\left(\mathbf{n}^{(0)}\right)\right] \\
+ & \delta^{2}\left[\mathcal{L} \cdot \mathbf{n}^{(2)}+\frac{1}{2} \mathcal{D}^{2} \mathcal{C}^{(0)}\left(\mathbf{n}^{(0)}\right) \cdot \mathbf{n}^{(1)} \mathbf{n}^{(1)}+\mathcal{D} \mathcal{C}^{(1)}\left(\mathbf{n}^{(0)}\right) \cdot \mathbf{n}^{(1)}\right] \\
+ & \delta^{3}\left[\mathcal{L} \cdot \mathbf{n}^{(3)}+\mathcal{D}^{2} \mathcal{C}^{(0)}\left(\mathbf{n}^{(0)}\right) \cdot \mathbf{n}^{(1)} \mathbf{n}^{(2)}+\frac{1}{6} \mathcal{D}^{3} \mathcal{C}^{(0)}\left(\mathbf{n}^{(0)}\right) \cdot \mathbf{n}^{(1)} \mathbf{n}^{(1)} \mathbf{n}^{(1)}\right. \\
& \left.\quad+\frac{1}{2} \mathcal{D}^{2} \mathcal{C}^{(1)}\left(\mathbf{n}^{(0)}\right) \cdot \mathbf{n}^{(1)} \mathbf{n}^{(1)}+\mathcal{D} \mathcal{C}^{(1)}\left(\mathbf{n}^{(0)}\right) \cdot \mathbf{n}^{(2)}\right] \\
+ & \delta^{4}\left[\mathcal{L} \cdot \mathbf{n}^{(4)}+\frac{1}{2} \mathcal{D}^{2} \mathcal{C}^{(0)}\left(\mathbf{n}^{(0)}\right) \cdot \mathbf{n}^{(2)} \mathbf{n}^{(2)}+\mathcal{D}^{2} \mathcal{C}^{(0)}\left(\mathbf{n}^{(0)}\right) \cdot \mathbf{n}^{(1)} \mathbf{n}^{(3)}\right. \\
& \quad+\frac{1}{2} \mathcal{D}^{3} \mathcal{C}^{(0)}\left(\mathbf{n}^{(0)}\right) \cdot \mathbf{n}^{(1)} \mathbf{n}^{(1)} \mathbf{n}^{(2)}+\frac{1}{24} \mathcal{D}^{4} \mathcal{C}^{(0)}\left(\mathbf{n}^{(0)}\right) \cdot \mathbf{n}^{(1)} \mathbf{n}^{(1)} \mathbf{n}^{(1)} \mathbf{n}^{(1)} \\
& \quad+\frac{1}{6} \mathcal{D}^{3} \mathcal{C}^{(1)}\left(\mathbf{n}^{(0)}\right) \cdot \mathbf{n}^{(1)} \mathbf{n}^{(1)} \mathbf{n}^{(1)}+\mathcal{D} \mathcal{D}^{2} \mathcal{C}^{(1)}\left(\mathbf{n}^{(0)}\right) \cdot \mathbf{n}^{(1)} \mathbf{n}^{(2)} \\
& \left.\quad+\mathcal{D} \mathcal{C}^{(1)}\left(\mathbf{n}^{(0)}\right) \cdot \mathbf{n}^{(3)}\right] \\
& +\mathcal{O}\left[\delta^{5}\right] \cdot
\end{aligned}
$$

This expansion is independent of the particular collision operator (up to $\mathcal{O}[\delta]$ advection Liasing, as per the discussion at the beginning of Section 2.3.2). Note that the form of the expansion depends neither on the dimensionality of the lattice nor on the number of particle directions. 


\subsubsection{Advection Operator Expansion}

The advection operator expansion depends on the dimensionality of the lattice and the number of particle directions. It also depends on the relationship between $\Delta x$ and $\Delta t . A s$ the focus of this dissertation is on systems with diffusion, we restrict treatment of the advection operator expansion to the case $\Delta t=\mathcal{O}\left[(\Delta x)^{2}\right]$, i.e., $\Delta t=T \delta^{2}$ and $\Delta x=L \delta$. The steps for determining the expansion follow:

1. Calculate the $(p+1)$-dimensional Taylor expansion of $\mathcal{A}_{k}(\vec{x} ; t)=n_{k}(\vec{x}+$ $\left.\Delta x \vec{e}_{k} ; t+\Delta t\right)$ evaluated at $(\vec{x} ; t)$ with $\Delta t=\delta^{2} T$ and $\Delta x=L \delta$. The constants $T$ and $L$ are the temporal and spatial scale lengths, respectively.

2. Substitute the space-gradient equilibrium expansion for $\mathrm{n}$ (see Eq. 2.14) into the result of step 1, writing the result as an expansion in $\delta$.

Let us apply these steps to determine explicit expressions for the advection operator expansion. But first, let us discuss gradient expressions as they appear in the advertion operator expansion, then we will proceed with the development of the expansion.

\section{Some Derivative Operations in the Advection Operator}

Before developing the advection expansion, some notation for gradients of vector valued functions of $m$ variables $\left(x_{0}, x_{1}, \ldots, x_{m-1}\right)$ needs to be introduced. If $\vec{e}_{k}$ is a unit velocity vector in direction $k$ on the lattice (and $c_{k, i}$ is its $i$ th component), then in operator notation

$$
\left(\vec{e}_{k} \cdot \nabla\right)^{j} \equiv\left(\sum_{i=0}^{m-1} e_{k, 1} \frac{\partial}{\partial x_{i}}\right)^{\prime} .
$$

As usual, expressions involving subscripts surrounded ly square brackets denote veretors compatible with vectors of occupation mumbers, i.e., vectors in $[0,1]^{d}$. 
Example ( $L B_{1}$ : Derivatives in Advection Operator).

Let us list some derivative expressions involving the four unit vectors in $L B_{1}$ as given in Eq. 2.4. Here, we are denoting $x_{0}=x$ and $x_{1}=y$. First,

$$
\begin{aligned}
& \text { 1. } \nabla=\left(\frac{\partial}{\partial x}, \frac{\partial}{\partial y}\right), \text { and } \\
& \text { 2. } \nabla^{2} \quad \nabla \cdot \nabla=\frac{\partial^{2}}{\partial x^{2}}+\frac{\partial^{2}}{\partial y^{2}} .
\end{aligned}
$$

Then

$$
\begin{aligned}
& \vec{e}_{0} \cdot \nabla=+\frac{\partial}{\partial x}, \quad\left(\vec{e}_{0} \cdot \nabla\right)^{2}=+\frac{\partial^{2}}{\partial x^{2}}, \quad\left(\vec{e}_{0} \cdot \nabla\right)^{3}=+\frac{\partial^{3}}{\partial x^{3}}, \\
& \vec{e}_{1} \cdot \nabla=+\frac{\partial}{\partial y}, \quad\left(\vec{e}_{1} \cdot \nabla\right)^{2}=+\frac{\partial^{2}}{\partial y^{2}}, \quad\left(\vec{e}_{1} \cdot \nabla\right)^{3}=+\frac{\partial^{3}}{\partial y^{3}}, \\
& \vec{e}_{2} \cdot \nabla=-\frac{\partial}{\partial x}, \quad\left(\vec{e}_{2} \cdot \nabla\right)^{2}=+\frac{\partial^{2}}{\partial x^{2}}, \quad\left(\vec{e}_{2} \cdot \nabla\right)^{3}=-\frac{\partial^{3}}{\partial x^{3}}, \\
& \vec{e}_{3} \cdot \nabla=-\frac{\partial}{\partial y}, \quad\left(\vec{e}_{3} \cdot \nabla\right)^{2}=+\frac{\partial^{2}}{\partial y^{2}}, \quad\left(\vec{e}_{3} \cdot \nabla\right)^{3}=-\frac{\partial^{3}}{\partial y^{3}} .
\end{aligned}
$$

in our vector notation these are written,

$$
\left[\vec{e}_{k} \cdot \nabla\right]=\left[\begin{array}{c}
+\frac{\partial}{\partial x} \\
+\frac{\partial}{\partial y} \\
-\frac{\partial}{\partial x} \\
-\frac{\partial}{\partial y}
\end{array}\right],\left[\left(\vec{e}_{k} \cdot \nabla\right)^{2}\right]=\left[\begin{array}{c}
+\frac{\partial^{2}}{\partial x^{2}} \\
+\frac{\partial^{2}}{\partial y^{2}} \\
+\frac{\partial^{2}}{\partial x^{2}} \\
+\frac{\partial^{2}}{\partial y^{2}}
\end{array}\right],\left[\left(\vec{e}_{k} \cdot \nabla\right)^{3}\right]=\left[\begin{array}{c}
+\frac{\partial^{3}}{\partial x^{3}} \\
+\frac{\partial^{3}}{\partial y^{3}} \\
-\frac{\partial^{3}}{\partial x^{3}} \\
-\frac{\partial^{3}}{\partial y^{3}}
\end{array}\right] .
$$

\section{Taylor Expansion}

First, let us recall the Taylor series expansion for a vector function of several independent variables. Let $\mathrm{n} \in C^{\infty}\left(\mathcal{R}^{m}, \mathcal{R}^{d}\right)$ be analytic in $\mathcal{R}^{m}$, where $\mathrm{n}(\vec{x})$ has $m$ 
independent variables, $\vec{x}=\left(x_{0}, x_{1}, \ldots, x_{m-1}\right)$, and $d$ cependent ones, $\mathbf{n}=\left[n_{k}\right]_{k=0}^{d-1}$ Then the Taylor series expansion about $\vec{\xi}=\left(x_{0}, x_{1}, \ldots, x_{m-1}\right)$ and evaluated at $\vec{\xi}+\vec{\gamma}_{k}$ is

$$
\left[n_{k}\left(\vec{\xi}+\vec{\gamma}_{k}\right)\right]_{k=0}^{m-1}=\left[\sum_{j=0}^{\infty} \frac{1}{j !}\left(\vec{\gamma}_{k} \cdot \nabla\right)^{j} n_{k}(\vec{\xi})\right]_{k=0}^{m-1},
$$

where $\vec{\gamma}_{k}$ is some vector distance away irom $\vec{\xi}$ that depends on which function, i.e., which $\left(n_{k}\right)$, is being evaluated. Here, we are using the usual definition of the gradient,

$$
\nabla \equiv\left(\frac{\partial}{\partial x_{0}}, \frac{\partial}{\partial x_{1}}, \ldots, \frac{\partial}{\partial x_{m-1}}\right)
$$

Also,

$$
\left(\vec{\gamma}_{k} \cdot \nabla\right)^{j} \equiv\left(\sum_{l=0}^{m-1} \gamma_{k, l} \frac{\partial}{\partial x_{l}}\right)^{j}
$$

The advection operator expansion comes from a special case of Eq. 2.20.

A lattice method has $d$ directions at each node of its lattice. It also has $p$ spatial dimensions with one temporal dimension. We use a Taylor expansion with $\mathbf{n}$ being the vector of occupation numbers (each a function of space/time position $\vec{\xi}$ ). The spatial/temporal positions are indicated by $\vec{\xi}=\left(x_{0}, x_{1}, \ldots, x_{p-1} ; t\right) \equiv(\vec{x} ; t)$. The desired Taylor expansion is of a vector function with $p+1$ independent variables, i.e., $(\vec{x} ; t)$, and $d$ dependent ones, i.e., $\mathbf{n}=\left[n_{0}, n_{1}, \ldots, n_{d-1}\right]^{T}$ ). The Taylor expansion may be written

$$
\begin{aligned}
{\left[\mathcal{A} n_{k}(\vec{x} ; t)\right] } & =\left[n_{k}\left(\vec{x}+\Delta x \vec{e}_{k} ; t+\Delta t\right)\right] \\
& =\left[\sum_{j=0}^{\infty} \frac{1}{j !}\left(\Delta x\left(\vec{e}_{k} \cdot \nabla\right)+\Delta t \frac{\partial}{\partial t}\right)^{j} n_{k}\right]
\end{aligned}
$$




$$
=\left[n_{k}(\vec{x} ; t)+\sum_{j=1}^{\infty} \frac{1}{j !}\left(L \delta\left(\vec{e}_{k} \cdot \nabla\right)+T \delta^{2} \frac{\partial}{\partial t}\right)^{j} n_{k}\right]
$$

where

$$
\begin{aligned}
\Delta x & \equiv L \delta \\
\Delta t & \equiv T \delta^{2},
\end{aligned}
$$

$\vec{e}_{k}$ is the unit velocity vector in direction $k$, and the gradient operator, $\nabla$, includes only the first $p$ independent variables, i.e.,

$$
\nabla \equiv\left(\frac{\partial}{\partial x_{0}}, \frac{\partial}{\partial x_{1}}, \ldots, \frac{\partial}{\partial x_{p-1}}\right)
$$

In particular the gradient does not include the temporal variable, $t$; it is treated separately due to the differing spatial and temporal scales. Rearranging Eq. 2.21 yields

$$
\begin{aligned}
& {\left[\mathcal{A} n_{k}(\vec{x} ; t)-n_{k}(\vec{x} ; t)\right]} \\
& =\delta\left[L\left(\vec{e}_{k} \cdot \nabla\right) n_{k}\right] \\
& +\delta^{2}\left[\frac{L^{2}}{2 !}\left(\vec{e}_{k} \cdot \nabla\right)^{2} n_{k}+T \frac{\partial}{\partial t} n_{k}\right] \\
& +\delta^{3}\left[\frac{L^{3}}{3 !}\left(\vec{e}_{k} \cdot \nabla\right)^{3} n_{k}+L T\left(\vec{e}_{k} \cdot \nabla\right) \frac{\partial}{\partial t} n_{k}\right] \\
& +\delta^{4}\left[\frac{L^{4}}{4 !}\left(\vec{e}_{k} \cdot \nabla\right)^{4} n_{k}+\frac{L^{2} T}{2}\left(\vec{e}_{k} \cdot \nabla\right)^{2} \frac{\partial}{\partial t} n_{k}+\frac{T^{2}}{2} \frac{\partial^{2}}{\partial t^{2}} n_{k}\right]
\end{aligned}
$$




$$
\begin{aligned}
& +\delta^{5}\left[\frac{L^{5}}{5 !}\left(\vec{e}_{k} \cdot \nabla\right)^{5} n_{k}+\frac{L T^{2}}{2}\left(\vec{e}_{k} \cdot \nabla\right) \frac{\partial^{2}}{\partial t^{2}} n_{k}+\frac{L^{3} T}{6}\left(\vec{e}_{k} \cdot \nabla\right)^{3} \frac{\partial}{\partial t} n_{k}\right] \\
& +\mathcal{O}\left[\delta^{6}\right] .
\end{aligned}
$$

This is the Taylor expansion of the advection operator. It expands the occupation numbers about a location $(\vec{x} ; t)$ and is evaluated at their new locations.

\section{Asymptotic Expansion}

The final expansion of the advection operator is finally obtained by substituting the asymptotic equilibrium expansion for $\mathrm{n}$ (see Eq. 2.14) into Eq. 2.23 and determining the coefficients of powers of $\delta$ :

$$
\begin{aligned}
& \begin{array}{l}
\left.\mathcal{A} n_{k}(\vec{x} ; t)-n_{k}(\vec{x} ; t)\right]= \\
\delta\left[L\left(\vec{e}_{k} \cdot \nabla\right) n_{k}^{(0)}\right] \\
+\delta^{2}\left[\frac{L^{2}}{2 !}\left(\vec{e}_{k} \cdot \nabla\right)^{2} n_{k}^{(0)}+T \frac{\partial}{\partial t} n_{k}^{(0)}+L\left(\vec{e}_{k} \cdot \nabla\right) n_{k}^{(1)}\right] \\
+\delta^{3}\left[\frac{L^{3}}{3 !}\left(\vec{e}_{k} \cdot \nabla\right)^{3} n_{k}^{(0)}+L T\left(\vec{e}_{k} \cdot \nabla\right) \frac{\partial}{\partial t} n_{k}^{(0)}+L\left(\vec{e}_{k} \cdot \nabla\right) n_{k}^{(2)}\right. \\
\left.+\frac{L^{2}}{2 !}\left(\vec{e}_{k} \cdot \nabla\right)^{2} n_{k}^{(1)}+T \frac{\partial}{\partial t} n_{k}^{(1)}\right] \\
+\delta^{4}\left[\frac{L^{4}}{4 !}\left(\vec{e}_{k} \cdot \nabla\right)^{4} n_{k}^{(0)}+\frac{L^{2} T}{2}\left(\vec{e}_{k} \cdot \nabla\right)^{2} \frac{\partial}{\partial t} n_{k}^{(0)}+\frac{T^{2}}{2} \frac{\partial^{2}}{\partial t^{2}} n_{k}^{(0)}+L\left(\vec{e}_{k} \cdot \nabla\right) n_{k}^{(3)}\right.
\end{array}
\end{aligned}
$$




$$
\begin{aligned}
& \left.+\frac{L^{2}}{2 !}\left(\vec{e}_{k} \cdot \nabla\right)^{2} n_{k}^{(2)}+T \frac{\partial}{\partial t} n_{k}^{(2)}+\frac{L^{3}}{3 !}\left(\vec{e}_{k} \cdot \nabla\right)^{3} n_{k}^{(1)}+L T\left(\vec{e}_{k} \cdot \nabla\right) \frac{\partial}{\partial t} n_{k}^{(1)}\right] \\
& +\delta^{5}\left[\frac{L^{5}}{5 !}\left(\vec{e}_{k} \cdot \nabla\right)^{5} n_{k}^{(0)}+\frac{L T^{2}}{2}\left(\vec{e}_{k} \cdot \nabla\right) \frac{\partial^{2}}{\partial t^{2}} n_{k}^{(0)}+\frac{L^{3}}{6}\left(\vec{e}_{k} \cdot \nabla\right)^{3} \frac{\partial}{\partial t} n_{k}^{(0)}\right. \\
& +\frac{L^{4}}{4 !}\left(\vec{e}_{k} \cdot \nabla\right)^{4} n_{k}^{(1)}+\frac{L^{2} T}{2}\left(\vec{e}_{k} \cdot \nabla\right)^{2} \frac{\partial}{\partial t} n_{k}^{(1)}+\frac{T^{2}}{2} \frac{\partial^{2}}{\partial t^{2}} n_{k}^{(1)} \\
& \left.+\frac{L^{3}}{3 !}\left(\vec{e}_{k} \cdot \nabla\right)^{3} n_{k}^{(2)}+L T\left(\vec{e}_{k} \cdot \nabla\right) \frac{\partial}{\partial t} n_{k}^{(2)}+\frac{L^{2}}{2 !}\left(\vec{e}_{k} \cdot \nabla\right)^{2} n_{k}^{(3)}+L\left(\vec{e}_{k} \cdot \nabla\right) n_{k}^{(4)}\right] \\
& +\mathcal{O}\left[\delta^{6}\right] .
\end{aligned}
$$

\subsubsection{Matching the Expansions}

Matching coefficients of like order of the advection and collision operator asymptotic exparsions (Eq. 2.24 and Eq. 2.18, respectively), we obtain the following hierarchy of equations for orders up to $\mathcal{O}\left[\delta^{4}\right]$ :

$\mathcal{O}[1]$

$$
\mathcal{C}^{(0)}\left(\mathbf{n}^{(0)}\right)=0
$$

$\mathcal{O}[\delta]:$

$$
\mathcal{L} \cdot \mathbf{n}^{(1)}+\mathcal{C}^{(1)}\left(\mathbf{n}^{(0)}\right)=\left[L\left(\vec{e}_{k} \cdot \nabla\right) n_{k}^{(0)}\right]
$$

$\mathcal{O}\left[\delta^{2}\right]:$

$$
\begin{aligned}
\mathcal{L} & \cdot \mathbf{n}^{(2)}+\frac{1}{2} \mathcal{D}^{2} \mathcal{C}^{(0)}\left(\mathbf{n}^{(0)}\right) \cdot \mathbf{n}^{(1)} \mathbf{n}^{(1)}+\mathcal{D} \mathcal{C}^{(1)}\left(\mathbf{n}^{(0)}\right) \cdot \mathbf{n}^{(1)} \\
& =\left[\frac{L^{2}}{2 !}\left(\vec{e}_{k} \cdot \nabla\right)^{2} n_{k}^{(0)}+T \frac{\partial}{\partial t} n_{k}^{(0)}+L\left(\vec{e}_{k} \cdot \nabla\right) n_{k}^{(1)}\right] .
\end{aligned}
$$


$\left(\delta^{3}\right]:$

$$
\begin{aligned}
\mathcal{L} & \cdot \mathbf{n}^{(3)}+\frac{1}{6} \mathcal{D}^{3} \mathcal{C}^{(0)}\left(\mathbf{n}^{(0)} \cdot n^{(1)} \mathbf{n}^{(1)} \mathbf{n}^{(1)}+\mathcal{D}^{2} \mathcal{C}^{(0)}\left(\mathbf{n}^{(0)}\right) \cdot \mathbf{n}^{(1)} \mathbf{n}^{(2)}\right. \\
+ & \frac{1}{2} \mathcal{D}^{2} \mathcal{C}^{(1)}\left(\mathbf{n}^{(0)}\right) \cdot \mathbf{n}^{(2)} \cdot 2^{(1)}+6 \mathcal{L}^{(1)}\left(\mathbf{n}^{(0)}\right) \cdot \mathbf{n}^{(2)} \\
= & {\left[\frac{L^{3}}{3 !} \cdot \vec{e}_{k} \cdot \nabla\right)^{3} n_{k}^{(0)}+L T\left(\vec{e}_{k} \cdot \nabla\right) \frac{\partial}{\partial t} n_{k}^{(0)}+L\left(\vec{e}_{k} \cdot \nabla\right) n_{k}^{(2)} } \\
+ & \left.\frac{L^{2}}{2 !}\left(\vec{e}_{k} \cdot \nabla\right)^{2} n_{k}^{(1)}+T \frac{\partial}{\partial t} n_{k}^{(1)}\right] .
\end{aligned}
$$

$\mathcal{O}\left[\delta^{4}\right]:$

$$
\begin{aligned}
\mathcal{L} & \cdot \mathbf{n}^{(4)}+\frac{1}{24} \mathcal{D}^{4} \mathcal{C}^{(0)}\left(\mathbf{n}^{(0)}\right) \cdot \mathbf{n}^{(1)} \mathbf{n}^{(1)} \mathbf{n}^{(1)} \mathbf{n}^{(1)}+\frac{1}{2} \mathcal{D}^{3} \mathcal{C}^{(0)}\left(\mathbf{n}^{(0)}\right) \cdot \mathbf{n}^{(1)} \mathbf{n}^{(1)} \mathbf{n}^{(2)} \\
& +\mathcal{D}^{2} \mathcal{C}^{(0)}\left(\mathbf{n}^{(0)}\right) \cdot \mathbf{n}^{(1)} \mathbf{n}^{(3)}+\frac{1}{2} \mathcal{D}^{2} \mathcal{C}^{(0)}\left(\mathbf{n}^{(0)}\right) \cdot \mathbf{n}^{(2)} \mathbf{n}^{(2)} \\
& +\frac{1}{6} \mathcal{D}^{3} \mathcal{C}^{(1)}\left(\mathbf{n}^{(0)}\right) \cdot \mathbf{n}^{(1)} \mathbf{n}^{(1)} \mathbf{n}^{(1)}+\mathcal{D}^{2} \mathcal{C}^{(1)}\left(\mathbf{n}^{(0)}\right) \cdot \mathbf{n}^{(1)} \mathbf{n}^{(2)}+\frac{1}{2} \mathcal{D} \mathcal{C}^{(1)}\left(\mathbf{n}^{(0)}\right) \cdot \mathbf{n}^{(3)} \\
& =\left[\frac{L^{4}}{4 !}\left(\vec{e}_{k} \cdot \nabla\right)^{4} n_{k}^{(0)}+\frac{L^{2} T}{2}\left(\vec{e}_{k} \cdot \nabla\right)^{2} \frac{\partial}{\partial t} n_{k}^{(0)}+\frac{T^{2}}{2} \frac{\partial^{2}}{\partial t^{2}} n_{k}^{(0)}+L\left(\vec{e}_{k} \cdot \nabla\right) n_{k}^{(3)}\right. \\
& \left.+\frac{L^{2}}{2 !}\left(\vec{e}_{k} \cdot \nabla\right)^{2} n_{k}^{(2)}+T \frac{\partial}{\partial t} n_{k}^{(2)}+\frac{L^{3}}{3 !}\left(\vec{e}_{k} \cdot \nabla\right)^{3} n_{k}^{(1)}+L T\left(\vec{e}_{k} \cdot \nabla\right) \frac{\partial}{\partial t} n_{k}^{(1)}\right]
\end{aligned}
$$

In managing this hierarchy of equations, it is generally convenient to express vectors of the form

$$
\left[\left(\vec{e}_{k} \cdot \nabla\right)^{j} n_{k}\right]
$$

as linear combinations of the eigenvectors of the linearized collision operator, $\mathcal{L}$. This, of course, depends upon the particular lattice method, its collision rules, the lattice, and the number of directions. But intelligently parameterizing lattice methods can 
significantly reduce the analysis of similar methods. ${ }^{25}$

\section{Example (LB 1 : Gradient Expressions).}

For $L B_{1}$, expressing $\left[\left(\vec{e}_{i} \cdot \nabla\right)^{k} n_{i}\right]$ as linear combinations of eigenvectors $(\mathcal{L})$ depends on whether $j$ is even or odd:

$$
\begin{aligned}
& {\left[\left(\vec{e}_{k} \cdot \nabla\right)^{j} n_{k}\right]=} \\
& \begin{cases}\left(\mathbf{q}_{1} \star \frac{\partial^{j}}{\partial x^{j}}+\mathbf{q}_{2} \star \frac{\partial^{j}}{\partial y^{j}}\right) \mathbf{n}, & j \text { odd }, \\
\left.\frac{1}{2} \mathbf{q}_{0} \star\left(\frac{\partial^{j}}{\partial x^{j}}+\frac{\partial^{j}}{\partial y^{j}}\right)+\frac{1}{2} \mathbf{q}_{3} \star\left(\frac{\partial^{j}}{\partial x^{j}}-\frac{\partial^{j}}{\partial y^{j}}\right)\right) \mathbf{n}, & j \text { even. } .\end{cases}
\end{aligned}
$$

Then, for example,

$$
\begin{aligned}
{\left[\left(\vec{e}_{k} \cdot \nabla\right) n_{k}^{(0)}\right] } & =\left(\mathbf{q}_{1} \star \frac{\partial}{\partial x}+\mathbf{q}_{2} \star \frac{\partial}{\partial y}\right) \mathbf{n}^{(0)} \\
& =\mathbf{q}_{1} \star \frac{\partial}{\partial x} \mathbf{n}^{(0)}+\mathbf{q}_{2} \star \frac{\partial}{\partial y} \mathbf{n}^{(0)} \\
& =\mathbf{q}_{1} \frac{\partial}{\partial x} u+\mathbf{q}_{2} \frac{\partial}{\partial y} u
\end{aligned}
$$

and ${ }^{26}$

$$
\begin{aligned}
& {\left[\left(\vec{e}_{k} \cdot \nabla\right)^{2} n_{k}^{(0)}\right]} \\
& =\left(\frac{1}{2} \mathbf{q}_{0} \star\left(\frac{\partial^{2}}{\partial x^{2}}+\frac{\partial^{2}}{\partial y^{2}}\right)+\frac{1}{2} \mathbf{q}_{3} \star\left(\frac{\partial^{2}}{\partial x^{2}}-\frac{\partial^{2}}{\partial y^{2}}\right)\right) \mathbf{n}^{(0)} \\
& =\frac{1}{2} \mathbf{q}_{0} \star\left(\frac{\partial^{2}}{\partial x^{2}} \mathbf{n}^{(0)}+\frac{\partial^{2}}{\partial y^{2}} \mathbf{n}^{(0)}\right)+\frac{1}{2} \mathbf{q}_{3} \star\left(\frac{\partial^{2}}{\partial x^{2}} \mathbf{n}^{(0)}-\frac{\partial^{2}}{\partial y^{2}} \mathbf{n}^{(0)}\right) \\
& =\frac{1}{2} \mathbf{q}_{0} \star\left(\frac{\partial^{2}}{\partial x^{2}} \mathbf{q}_{0}+\frac{\partial^{2}}{\partial y^{2}} \mathbf{q}_{0}\right) u+\frac{1}{2} \mathbf{q}_{3} \star\left(\frac{\partial^{2}}{\partial x^{2}} \mathbf{q}_{0}-\frac{\partial^{2}}{\partial y^{2}} \mathbf{q}_{0}\right) u \\
& =\frac{1}{2} \mathbf{q}_{0}\left(\frac{\partial^{2}}{\partial x^{2}}+\frac{\partial^{2}}{\partial y^{2}}\right) u+\frac{1}{2} \mathbf{q}_{3}\left(\frac{\partial^{2}}{\partial x^{2}}-\frac{\partial^{2}}{\partial y^{2}}\right) u .
\end{aligned}
$$

\footnotetext{
25 "Similar" means having the same lattice dimensionality, number of particle directions, eigen vectors of the linearized collision operator, and nullspace of the linearized collision operator.

${ }^{26}$ Note the use of the $\star$ operation for componentwise vector product (see Section 2.1.1).
} 
(cf. Eq. 2.19, which lists operator $\left[\left(\vec{e}_{k} \cdot \nabla\right)^{2}\right]$ in a form without the eigenvectors.)

As previously motivated, the convenience of expressing $\left[\left(\vec{e}_{k} \cdot \nabla\right)^{j} n_{k}\right]$ as linear combinations of the eigenvectors of $\mathcal{L}$ comes from that the eigenvectors are mutually orthogonal. Then the componentwise vector product $(\star)$ can be employed to simplify complicated expressions. This is especially useful when the expressions involve dot products.

\section{Example ( $L B_{1}$ : Using Componentwise Eigenvector Products).}

Consider the expression $q_{0} \cdot\left(q_{1} \star q_{2}\right)$. Then using the $\star$ multiplication table (Table 2.4),

$$
\mathbf{q}_{0} \cdot\left(\mathbf{q}_{1} \star \mathbf{q}_{2}\right)=\mathbf{q}_{0} \cdot\left[\frac{1}{2}\left(\mathbf{q}_{0}+\mathbf{q}_{3}\right)\right]=\frac{1}{2}\left[\left(\mathbf{q}_{0} \cdot \mathbf{q}_{0}\right)+\left(\mathbf{q}_{0} \cdot \mathbf{q}_{3}\right)\right]=\frac{1}{2}[4+0]=2
$$

For the equilibrium expansion, Eq. 2.14, to exist (let alone converge), we must be able to obtain expressions for the $\mathrm{r}^{(k)}$. In matching the advection and collision operator expansions, these coefficients are obtained using the pseudo-inverse $\left(\mathcal{L}^{+}\right)$of the linearized collision operator (see Definition 2.34). At each order $j \geq 1$ in matching the expansions appears an equation, $\mathcal{L} \cdot \mathbf{n}^{(j)}=\mathbf{g}^{(j)}, \mathbf{n}^{(j)}, \mathbf{g}^{(j)} \in[0,1]^{d}$. In order that a solution exists, the r.h.s., i.e., $\mathbf{g}^{(j)}$, must be orthogonal to the nullspace of $\mathcal{L}$. This requirement forces a consistency or compatibility condition for each eigenvector in the nullspace of $\mathcal{L}$. 
Definition 2.35 (CONSISTEnCY Condition). Given that $\mathcal{L} \cdot \mathbf{n}^{(j)}=\mathbf{g}^{(i)}$ for some order $j$ in the discrete Chapman-Enskog expansion, the $j$ th order $\left(\mathcal{O}\left[\delta^{j}\right]\right)$, consistency or compatibility condition is that

$$
\mathbf{q}_{k} \cdot \mathbf{g}^{(j)}=0 \quad \forall k \ni \lambda_{k}=0
$$

where $\lambda_{k} \in$ eigenvalues $(\mathcal{L})$.

If the consistency condition holds, then $\mathbf{n}^{(j)}$ may be obtained $u$ sing $\mathcal{L}^{+}$, and $\mathbf{n}^{(j)}=$ $\mathcal{L}^{+} \cdot \mathbf{g}^{(j)}+\mathbf{a}$, in which a $\in \operatorname{span}($ nullspace $(\mathcal{L}))$ is arbitrary.

\section{Example (LB $B_{1}$ Form of $\mathcal{O}\left[\delta^{j}\right]$ Consistency Condition).}

For $L B_{1}, \operatorname{nullspace}(\mathcal{L})=\operatorname{span}\left(q_{0}\right)$. Then at $\left.\mathcal{C}\right)\left[\delta^{j}\right] \mathcal{L} \cdot \mathbf{n}^{(j)}=\mathbf{g}^{(j)}$ for some $\mathbf{g}^{(j)}$, which is known. The consistency condition is that $q_{0} \cdot g^{(i)}=0$. If the consistency condition holds, then $\mathbf{n}^{(j)}=\mathcal{L}^{+} \cdot \mathbf{g}^{(j)}+\sigma^{(j)} \mathbf{q}_{0}$, in which $\sigma^{(j)} \mathbf{q}_{0} \in$ unullspace $\left(L^{\prime}\right)$ is arbitrary $\left(\sigma^{(j)}\right.$ is a scalar $)$.

At each order, $\mathcal{O}\left[\delta^{j}\right]$, in matching the advection and collision operator expansions, we will solve for $n^{(j)}$ in terms of $\left\{n^{(1)}, \mathbf{n}^{(1)}, \ldots, \mathbf{n}^{(j-1)}\right\}$. 'This will introduce a consistency condition for each eigenvector in the nullspace of $\mathcal{L}$ at that order. It will also introduce the same number of arbitrary parameters, which are denoted $\left(\sigma_{0}^{(j)}, \sigma_{1}^{(j)}, \ldots\right){ }^{27}$

DEFinition 2.36 (Hydrodynamical Equation). If $\Delta x=\mathcal{O}[\delta]$ and $\Delta t=$ $\mathcal{O}\left[\delta^{2}\right]$, then the $\mathcal{O}\left[\delta^{2}\right]$ consistency condition is called the hydrodynamical ecfuation of a lattice method. When the $\mathcal{O}\left[\delta^{2}\right]$ consistency condition is a multiple cordition, i.e., the dimension of the nullspace of the linearized collision operator is greater than one, we refer to the multiple conditions as the hydrodynamical equations.

\footnotetext{
${ }^{27}$ If $\operatorname{dim}($ nullspace $(\mathcal{L}))=1$, we write $\sigma^{(j)}$ for $\sigma_{0}^{(j)}$, the subscript being unnecessary.
} 
Since only $\left\{n^{(0)}, n^{(1)}, \ldots, n^{,-1)}\right\}$ is needed to determine the consistency condition at order $j$, solving for $n^{(j)}$ only helps in determining the next higher order consistency condition. Now, let us perform these tasks for our example, $L_{1} B_{1}$, up to $\mathcal{O}\left[\delta^{5}\right]$. (Please note that as this will take up several pages, it is suggested that upon a first reading the reader refer to the important results, namely the $\mathcal{O}\left[\delta^{2}\right], \mathcal{O}\left[\delta^{3}\right]$, and $\mathcal{O}\left[\delta^{4}\right]$ consistency conditions, Eq. 2.29, Eq̨. 2.31, and Eq. 2.37, respectively. Also, while some details of the calculations are included in the text, the interested reader will find further details in Appendix A.)

\section{Example ( $L B_{1}$ : Applying the Discrete Chapman-Enskog Procedure).}

The following notation is used throughout this example:

$$
\begin{aligned}
\lambda & =\lambda_{1}=\lambda_{2}=\lambda_{3}=-4 u(1-u), \\
D(u) & =-\frac{1}{\lambda}-\frac{1}{2}, \\
D^{\prime}(u) & =\frac{4(2 u-1)}{\lambda^{2}}, \\
\mathbf{g}^{(j)} & =\mathcal{L} \cdot \mathbf{n}^{(j)}=\sum_{k=0}^{3} \tilde{c}_{k}^{(j)} \mathbf{q}_{k}, \\
\mathbf{n}^{(j)} & =\sigma^{(j)} \mathbf{q}_{0}+\mathcal{L}^{+} \cdot \mathbf{g}^{(j)}=\sum_{k=j^{\prime}}^{3} c_{k}^{(j)} \mathbf{q}_{k},
\end{aligned}
$$

in which $c_{0}^{(j)}=\sigma^{(j)}$ and $c_{k}^{(j)}=\frac{1}{\lambda_{k}} \tilde{c}_{k}^{(j)}$ for $k \in\{1,2,3\}$. Also, recall $\mathcal{C}(\mathbf{n})=\mathcal{C}^{(0)}(\mathbf{n})$.

In the forthcoming formal consistency and stability arguments, we will be using an equilibrium expansion truncated beyond third order, i.e., $\mathbf{h}=\sum_{j=0}^{3} \delta^{j} \mathbf{h}^{(j)}$, in which $\mathbf{h}^{(j)}=\mathbf{n}^{(j)}$. It turns out that $\sigma^{(3)}$ appears in $\mathbf{n}^{(3)}$, and $\sigma^{(3)}$ is determined by the $\mathcal{O}\left[\delta^{5}\right]$ consistency condition. Therefore, we must carry out the discrete Chapman-Enskog procedure to determine the consistency condition at $\mathcal{O}\left[\delta^{5}\right]$. For each order in matching the advection and collision operator expansions, Eq. 2.24 
and Eq. 2.18, respectively, we find the following:

$\mathcal{O}[1]: \mathcal{C}^{(0)}\left(\mathbf{n}^{(0)}\right)=\mathcal{C}\left(\mathbf{n}^{(0)}\right)=0$, as desired, by choice of $\mathbf{n}^{(0)}$. Then

$$
\mathbf{n}^{(0)}=\mathbf{q}_{0} u
$$

$\mathcal{O}[\delta]:$ We find

$$
\begin{aligned}
\mathcal{L} \cdot \mathbf{n}^{(1)} & =\left[L\left(\vec{e}_{k} \cdot \nabla\right) n_{k}^{(0)}\right] \\
& \equiv \mathbf{g}^{(1)} \\
& =\sum_{k=0}^{3} \tilde{c}_{k}^{(1)} \mathbf{q}_{k},
\end{aligned}
$$

where

$$
\begin{aligned}
& \tilde{c}_{0}^{(1)}=0, \\
& \tilde{c}_{1}^{(1)}=L u_{x}, \\
& \tilde{c}_{2}^{(1)}=L u_{y}, \\
& \tilde{c}_{3}^{(1)}=0
\end{aligned}
$$

by applying Eq. 2.25 .

- Consistency: $\mathbf{q}_{0} \cdot \mathbf{g}^{(1)}=0$ is already satisfied, so no consistency condition is introduced at this order.

- Solve for $\mathbf{n}^{(1)}$ : We have

$$
\begin{aligned}
\mathbf{n}^{(1)} & =\mathcal{L}^{+} \cdot \mathbf{g}^{(1)}+\sigma^{(1)} \mathbf{q}_{0} \\
& =c_{0}^{(1)} \mathbf{q}_{0}+\sum_{k=0}^{3} c_{k}^{(1)} \mathbf{q}_{k},
\end{aligned}
$$

where

$$
c_{0}^{(1)}=\sigma^{(1)}
$$


is a free parameter (determined in the $\mathcal{O}\left[\delta^{3}\right]$ consistency condition), and

$$
c_{k}^{(1)}=\frac{1}{\lambda_{k}} \pi_{k}^{(1)}, k \in\{1,2,3\},
$$

or

$$
\begin{aligned}
& c_{1}^{(1)}=L \frac{1}{\lambda} u_{x}, \\
& c_{2}^{(1)}=L \frac{1}{\lambda} u_{y}, \\
& c_{3}^{(1)}=0,
\end{aligned}
$$

in which $\lambda \equiv \lambda_{1}=\lambda_{2}=\lambda_{3}$ (see Eq. 2.15 for eigenvalues $(\mathcal{L})$ ).

$\mathcal{O}\left[\delta^{2}\right]:$ We find

$$
\begin{aligned}
\mathcal{L} \cdot \mathbf{n}^{(2)} & =\left[\frac{L^{2}}{2 !}\left(\vec{e}_{k} \cdot \nabla\right)^{2} n_{k}^{(0)}+T \frac{\partial}{\partial t} n_{k}^{(0)}+L\left(\vec{e}_{k} \cdot \nabla\right) n_{k}^{(1)}\right] \\
& -\frac{1}{2} \mathcal{D}^{2} \mathcal{C}^{\prime}\left(\mathbf{n}^{(0)}\right) \cdot \mathbf{n}^{(1)} \mathbf{n}^{(1)} \\
& \equiv \mathbf{g}^{(2)} \\
& =\frac{L^{2}}{2}\left(\frac{1}{2} \mathbf{q}_{0}\left(\frac{\partial^{2}}{\partial x^{2}} u+\frac{\partial^{2}}{\partial y^{2}} u\right)+\frac{1}{2} \mathbf{q}_{3}\left(\frac{\partial^{2}}{\partial x^{2}} u-\frac{\partial^{2}}{\partial y^{2}} u\right)\right) \\
& +\mathbf{q}_{0} T \frac{\partial}{\partial t} u+L\left(\mathbf{q}_{1} \star \frac{\partial}{\partial x}+\mathbf{q}_{2} \star \frac{\partial}{\partial y}\right)\left(c_{0}^{(1)} \mathbf{q}_{0}+c_{1}^{(1)} \mathbf{q}_{1}+c_{2}^{(1)} \mathbf{q}_{2}\right) \\
& -\frac{1}{2} \mathcal{D}^{2} \mathcal{C}\left(\mathbf{n}^{(0)}\right) \cdot \mathbf{n}^{(1)} \mathbf{n}^{(1)} \\
& =\sum_{\kappa=0}^{3} \dot{c}_{k}^{(2)} \mathbf{q}_{k},
\end{aligned}
$$

where

$$
\begin{aligned}
\tilde{c}_{0}^{(2)} & =T u_{t}-\frac{L^{2}}{2} \nabla \cdot D(u) \nabla u \\
\tilde{c}_{1}^{(2)} & =L \sigma_{x}^{(1)}-L D^{\prime}(u) \lambda \sigma^{(1)} u_{x} \\
\tilde{c}_{2}^{(2)} & =L \sigma_{y}^{(1)}-L D^{\prime}(u) \lambda \sigma^{(1)} u_{y}, \\
\tilde{c}_{3}^{(2)} & =\frac{L^{2}}{2}\left[\frac{1}{2} D^{\prime}(u)\left(\left(u_{x}\right)^{2}-\left(u_{y}\right)^{2}\right)-\left(\frac{\partial}{\partial x}\left(D(u) u_{x}\right)-\frac{\partial}{\partial y}\left(D(u) u_{y}\right)\right)\right] .
\end{aligned}
$$


- Consistency: Imposing the consistency condition that $\mathbf{q}_{0} \cdot \mathrm{g}^{(2)}=0$ yieids

$$
\mathbf{q}_{0} \cdot \sum_{j=0}^{3} \tilde{c}_{j}^{(2)} \mathbf{q}_{j}=4 \tilde{c}_{0}^{(2)}=0
$$

or

$$
\tilde{c}_{0}^{(2)}=0
$$

With $\nu \equiv \frac{L^{2}}{2 T}$ this may be written

$$
u_{t}=\nu \nabla \cdot D(u) \nabla u
$$

- Solve for $n^{(2)}$ : We find

$$
\begin{aligned}
\mathbf{n}^{(2)} & =\mathcal{L}^{+} \cdot \mathbf{g}^{(2)}+\sigma^{(2)} \mathbf{q u}_{0} \\
& =\sum_{k=0}^{3} c_{k}^{(2)} \mathbf{q}_{k},
\end{aligned}
$$

where

$$
c_{0}^{(2)}=\sigma^{(2)}
$$

is a free parameter (to be determined in the $\mathcal{O}\left[\delta^{d}\right]$ consistency condition), and

$$
c_{k}^{(2)}=\frac{1}{\lambda_{k}} \tilde{c}_{k}^{(2)}, k \in\{1,2,3\}
$$

or

$$
\begin{aligned}
c_{1}^{(2)} & =\frac{L}{\lambda} \sigma_{x}^{(1)}-L D^{\prime}(u) \lambda \sigma^{(1)} u_{x}, \\
c_{2}^{(2)} & =\frac{L}{\lambda} \sigma_{y}^{(1)}-L D^{\prime}(u) \lambda \sigma^{(1)} u_{y}, \\
c_{3}^{(2)} & \left.=\frac{L^{2}}{2 \lambda}\left[\frac{1}{2} D^{\prime}(u)\left(\left(u_{x}\right)^{2}-\left(u_{y}\right)^{2}\right)-\left(\frac{\partial}{\partial x}(D)(u) u_{x}\right)-\frac{\partial}{\partial y}\left(D(u) u_{y}\right)\right)\right] .
\end{aligned}
$$

$\mathcal{O}\left[\delta^{3}\right]:$ We find

$$
\mathcal{L} \cdot \mathbf{n}^{(3)}=\left[\frac{L^{3}}{3 !}\left(\vec{e}_{k} \cdot \nabla\right)^{3} n_{k}^{(0)}+L T\left(\vec{e}_{k} \cdot \nabla\right) \frac{\partial}{\partial t} n_{k}^{(0)}+L\left(\vec{e}_{k} \cdot \nabla\right) n_{k}^{(2)}\right.
$$




$$
\begin{aligned}
& \left.\quad+\frac{L^{2}}{2 !}\left(\vec{r}_{k} \cdot \nabla\right)^{2} n_{k}^{(1)}+T \frac{\partial}{\partial t} n_{k}^{(1)}\right] \\
& -D^{2} C^{\prime}\left(\mathbf{n}^{(())}\right) \cdot \mathbf{n}^{(1)} \mathbf{n}^{(2)}-\frac{1}{6} D^{3} C\left(\mathbf{n}^{(1)}\right) \cdot \mathbf{n}^{(1)} \mathbf{n}^{(1)} \mathbf{n}^{(1)} \\
& \equiv \mathbf{g}^{(3)} \\
& =\sum_{k=0}^{3} \tilde{c}_{k}^{(3)} \mathbf{q}_{k}
\end{aligned}
$$

where

$$
\begin{aligned}
& \tilde{c}_{0}^{(3)}=T \sigma_{l}^{(1)}-\frac{L^{2}}{2} \nabla \cdot\left[D(u) \nabla \sigma^{(1)}+D^{\prime}(u) \sigma^{(1)} \nabla u\right], \\
& \tilde{c}_{1}^{(3)}=\frac{L^{3}}{6} u_{x x x}+L T u_{x t}+\left(\frac{L^{3}}{2} \cdot \frac{\partial^{2}}{\partial x^{2}}+L T \frac{\partial}{\partial t}\right)\left(\frac{1}{\lambda} u_{x}\right) \\
& +\frac{L^{3}}{2} \frac{\partial}{\partial x}\left(\frac{1}{2 \lambda} D^{\prime}(u)\left(\left(u_{x}\right)^{2}-\left(u_{y}\right)^{2}\right)-\frac{1}{\lambda}\left(\frac{\partial}{\partial x}\left(D(u) u_{x}\right)-\frac{\partial}{\partial y}\left(D(u) u_{y}\right)\right)\right) \\
& +L \sigma_{x}^{(2)}-\frac{4 L}{\lambda^{3}} u_{x}\left(\left(\lambda \sigma^{(1)}\right)^{2}-\left(L u_{v}\right)^{2}\right) \\
& +\frac{L}{4} D^{\prime}(u)\left[\left(2 \lambda \sigma^{(1)}\right)^{2} D^{\prime}(u) u_{x}-4 \lambda\left(\sigma^{(2)} u_{x}+\sigma^{(1)} \sigma_{x}^{(1)}\right)\right. \\
& \left.+L^{2} u_{x}\left(D^{\prime}(u)\left(\left(u_{x}\right)^{2}-\left(u_{y}\right)^{2}\right)-2\left(\frac{\partial}{\partial x}\left(D(u) u_{x}\right)-\frac{\partial}{\partial y}\left(D(u) u_{v}\right)\right)\right)\right] \text {, } \\
& \tilde{c}_{2}^{(3)}=\frac{L^{3}}{6} u_{y y y}+L T u_{y t}+\left(\frac{L^{3}}{2} \frac{\partial^{2}}{\partial y^{2}}+L T \frac{\partial}{\partial t}\right)\left(\frac{1}{\lambda} u_{y}\right) \\
& -\frac{L^{3}}{2} \frac{\partial}{\partial y}\left(\frac{1}{2 \lambda} D^{\prime}(u)\left(\left(u_{x}\right)^{2}-\left(u_{y}\right)^{2}\right)-\frac{1}{\lambda}\left(\frac{\partial}{\partial x}\left(D(u) u_{x}\right)-\frac{\partial}{\partial y}\left(D(u) u_{y}\right)\right)\right) \\
& +\quad L \sigma_{y}^{(2)}-\frac{4 L}{\lambda^{3}} u_{y}\left(\left(\lambda \sigma^{(1)}\right)^{2}-\left(L u_{x}\right)^{2}\right) \\
& +\frac{L}{4} D^{\prime}(u)\left[\left(2 \lambda \sigma^{(1)}\right)^{2} D^{\prime}(u) u_{y}-4 \lambda\left(\sigma^{(2)} u_{y}+\sigma^{(1)} \sigma_{y}^{(1)}\right)\right. \\
& \left.-L^{2} u_{y}\left(D^{\prime}(u)\left(\left(u_{x}\right)^{2}--\left(u_{y}\right)^{2}\right)-2\left(\frac{\partial}{\partial x}\left(D(u) u_{x}\right)-\frac{\partial}{\partial y}\left(D(u) u_{y}\right)\right)\right)\right] \text {, } \\
& \tilde{r}_{3}^{(3)}=\frac{2 L^{2}}{\lambda^{2}} \sigma^{(1)}\left(\left(u_{x}\right)^{2}-\left(u_{y}\right)^{2}\right)+\frac{L^{2}}{2}\left(\frac{\partial}{\partial x},-\frac{\partial}{\partial y}\right) \cdot\left[D(u) \nabla \sigma^{(1)}+D^{\prime}(u) \sigma^{(1)} \nabla u\right] \\
& +\frac{L^{2}}{4} D^{\prime}(u)\left[2 \lambda \sigma ^ { ( 1 ) } \left(\frac{\partial}{\partial x}\left(D(u) u_{x} j-\frac{\partial}{\partial y}\left(D(u) u_{y}\right)\right)\right.\right. \\
& \left.-3 \lambda \sigma^{(1)} D^{\prime}(u)\left(\left(u_{x}\right)^{2}-\left(u_{y}\right)^{2}\right)+2\left(\sigma_{y}^{(1)} u_{x}+\sigma_{y}^{(1)} u_{y}\right)\right] \text {. }
\end{aligned}
$$


- Consistency: Imposing the consistency condition that $q_{0} \cdot g^{(3)}=0$ gives

$$
\tilde{c}_{0}^{(3)}=0
$$

or

$$
\sigma_{t}^{(1)}=\nu \nabla \cdot\left[D(u) \nabla \sigma^{(1)}+D^{\prime}(u) \sigma^{(1)} \nabla u\right] .
$$

Note that $\sigma^{(1)}(x, y ; t)=$ const. satisfies the consistency condition. Let us choose $\sigma^{(1)}(x, y ; t)=0$. This choice simplifies the $\mathcal{O}\left[\delta^{4}\right]$ calculations and is crucial for obtaining consistency and stability.

- Solve for $\mathbf{n}^{(3)}$ : We find

$$
\begin{aligned}
\mathbf{n}^{(3)} & =\mathcal{L}^{+} \cdot \mathbf{g}^{(3)}+\sigma^{(3)} \mathbf{q}_{0} \\
& =\sum_{k=0}^{3} c_{k}^{(3)} \mathbf{q}_{k},
\end{aligned}
$$

where

$$
c_{0}^{(3)}=\sigma^{(3)}
$$

is a free parameter (to be determined in the $\mathcal{O}\left[\delta^{5}\right]$ consistency condition), and

$$
c_{k}^{(3)}=\frac{1}{\lambda_{k}} \tilde{c}_{k}^{(3)}, k \in\{1,2,3\} .
$$

Note that if $\sigma^{(1)}(x, y ; t)=0$, then $\tilde{c}_{3}^{(3)}=c_{3}^{(3)}=0$. Further calculations in the derivations assume $\sigma^{(1)}=0$.

$\mathcal{O}\left[\delta^{4}\right]:$ We find

$$
\begin{aligned}
\mathcal{L} \cdot \mathbf{n}^{(4)} & \\
= & {\left[\frac{L^{4}}{4 !}\left(\vec{e}_{k} \cdot \nabla\right)^{4} n_{k}^{(0)}+\frac{L^{2} T}{2}\left(\vec{e}_{k} \cdot \nabla\right)^{2} \frac{\partial}{\partial t} n_{k}^{(0)}+\frac{T^{2}}{2} \frac{\partial^{2}}{\partial t^{2}} n_{k}^{(0)}+L\left(\vec{e}_{k} \cdot \nabla\right) n_{k}^{(3)}\right.} \\
& \left.\frac{L^{2}}{2 !}\left(\vec{e}_{k} \cdot \nabla\right)^{2} n_{k}^{(2)}+T \frac{\partial}{\partial t} n_{k}^{(2)}+\frac{L^{3}}{3 !}\left(\vec{e}_{k} \cdot \nabla\right)^{3} n_{k}^{(1)}+L T\left(\vec{e}_{k} \cdot \nabla\right) \frac{\partial}{\partial t} n_{k}^{(1)}\right]
\end{aligned}
$$




$$
\begin{aligned}
& -\frac{1}{24} \mathcal{D} \mathcal{D} \mathcal{C}\left(\mathbf{n}^{(0)}\right) \cdot \mathbf{n}^{(1)} \mathbf{n}^{(1)} \mathbf{n}^{(1)} \mathbf{n}^{(1)}-\frac{1}{2} \mathcal{D}^{3} \mathcal{C}\left(\mathbf{n}^{(0)}\right) \cdot \mathbf{n}^{(1)} \mathbf{n}^{(1)} \mathbf{n}^{(2)} \\
& -\mathcal{D}^{2} \mathcal{C}\left(\mathbf{n}^{(0)}\right) \cdot \mathbf{n}^{(1)} \mathbf{n}^{(3)}-\frac{1}{2} \mathcal{D}^{2} \mathcal{C}\left(\mathbf{n}^{(0)}\right) \cdot \mathbf{n}^{(2)} \mathbf{n}^{(2)} \\
& \equiv \mathbf{g}^{(4)} \\
& =\sum_{k=0}^{3} \tilde{c}_{k}^{(4)} \mathbf{q}_{k}
\end{aligned}
$$

where

$$
\begin{aligned}
\tilde{c}_{0}^{(4)}= & T \sigma_{t}^{(2)}+\frac{L^{4}}{48}\left(u_{x x x x}+u_{y y y y}\right)+\frac{L^{2} T}{4}\left(u_{x x t}+u_{y y t}\right) \\
+ & \frac{L^{4}}{12}\left[\frac{\partial^{3}}{\partial x^{3}}\left(\frac{1}{\lambda} u_{x}\right)+\frac{\partial^{3}}{\partial y^{3}}\left(\frac{1}{\lambda} u_{y}\right)\right]+\frac{L^{2} T}{2} \nabla \cdot \frac{\partial}{\partial t}\left(\frac{1}{\lambda} \nabla u\right) \\
+ & \frac{T^{2}}{2} u_{t t}+\frac{L}{2}\left(\frac{\partial}{\partial x} c_{1}^{(3)}+\frac{\partial}{\partial y} c_{2}^{(3)}\right)+\frac{L^{2}}{4}\left[\nabla^{2} \sigma^{(2)}+\left(\frac{\partial^{2}}{\partial x^{2}}-\frac{\partial^{2}}{\partial y^{2}}\right) c_{3}^{(2)}\right], \\
\tilde{c}_{1}^{(4)}= & L\left(\sigma_{x}^{(3)}-D^{\prime}(u) \lambda \sigma^{(3)} u_{x}\right), \\
\tilde{c}_{2}^{(4)}= & L\left(\sigma_{y}^{(3)}-D^{\prime}(u) \lambda \sigma^{(3)} u_{y}\right), \\
\tilde{c}_{3}^{(4)}= & \frac{L^{4}}{48}\left(u_{x x x x}-u_{y y y y}\right)+\frac{L^{2} T}{4}\left(u_{x x t}-u_{y y t}\right)+T \frac{\partial}{\partial t} c_{3}^{(2)} \\
+ & \frac{L^{2}}{4}\left[\sigma_{x x}^{(2)}-\sigma_{y y}^{(2)}+\left(\frac{\partial^{2}}{\partial x^{2}}+\frac{\partial^{2}}{\partial y^{2}}\right) c_{3}^{(2)}\right]+\frac{L}{2}\left(\frac{\partial}{\partial x} c_{1}^{(3)}-\frac{\partial}{\partial y} c_{2}^{(3)}\right) \\
+ & \frac{L^{4}}{12}\left(\frac{\partial^{3}}{\partial x^{3}},-\frac{\partial^{3}}{\partial y^{3}}\right) \cdot \frac{\partial}{\partial t}\left(\frac{1}{\lambda} \nabla u\right)+\frac{L^{2} T}{2}\left(\frac{\partial}{\partial x},-\frac{\partial}{\partial y}\right) \cdot \frac{\partial}{\partial t}\left(\frac{1}{\lambda} \nabla u\right) \\
- & \frac{L^{2}}{\lambda^{3}}\left[\frac{L^{2}}{2} D^{\prime}(u)\left(\left(u_{x}\right)^{4}-\left(u_{y}\right)^{4}\right)\right. \\
& \left.-L^{2}\left(\left(u_{x}\right)^{2}+\left(u_{y}\right)^{2}\right)\left(\frac{\partial}{\partial x},-\frac{\partial}{\partial y}\right) \cdot D(u) \nabla u-2 \lambda \sigma^{(2)}\left(\left(u_{x}\right)^{2}-\left(u_{y}\right)^{2}\right)\right] \\
- & \frac{L^{2}}{4} D^{\prime}(u) \lambda \sigma^{(2)}\left[D^{\prime}(u)\left(\left(u_{x}\right)^{2}-\left(u_{y}\right)^{2}\right)-2\left(\frac{\partial}{\partial x},-\frac{\partial}{\partial y}\right) \cdot D(u) \nabla u\right] \\
+ & \frac{L}{2} D^{\prime}(u) \lambda\left(c_{1}^{(3)} u_{x}-c_{2}^{(3)} u_{y}\right) \cdot \\
= &
\end{aligned}
$$

- Consistency: Imposing the consistency condition that $\mathrm{q}_{0} \cdot \mathrm{g}^{(4)}=0$, we find

$$
\begin{aligned}
\sigma_{t}^{(2)} & =\nu \nabla \cdot\left[D(u) \nabla \sigma^{(2)}+D^{\prime}(u) \sigma^{(2)} \nabla u\right] \\
& -\mathcal{F}\left(L, T ; \tilde{w}, y, t ; u, u_{x_{i}}, u_{x_{i} x_{j}}, u_{x_{i} x_{j} x_{k}}, u_{x_{i} x_{j} x_{k} x_{l}}\right)
\end{aligned}
$$


where $x_{0}$ and $x_{1}$ denote $x$ and $y$, respectively. Note that $\mathcal{F}$ can be explicitly expressed in terms of the spatial derivatives of $u$ by using the results of this section and the details of Appendix $A$. (Note that the $\mathcal{O}\left[\delta^{2}\right]$ consistency condition, Eq. 2.29, can be used to remove dependences of $\mathcal{F}$ on the temporal derivatives of $u$.)

Note that Eq. 2.37 may be written

$$
(\mathbf{L}+\mathbf{h})\left[\sigma^{(2)}\right]=\mathcal{F}
$$

where

$$
\mathbf{L} \equiv \sum_{i, j=0}^{1} a_{i j}(\vec{x} ; t) \frac{\partial^{2}}{\partial x_{i} \partial x_{j}}+\sum_{i=0}^{1} b_{i}(\vec{x} ; t) \frac{\partial}{\partial x_{i}}-\frac{\partial}{\partial t}
$$

in which

$$
\begin{aligned}
a_{00}(x, y ; t) & =\nu D(u), \\
a_{01}(x, y ; t) & =0, \\
a_{10}(x, y ; t) & =0, \\
a_{11}(x, y ; t) & =\nu D(u) ; \\
b_{u}(x, y ; t) & =\nu D^{\prime}(u) u_{x}, \\
b_{1}(x, y ; t) & =\nu D^{\prime}(u) u_{y} ;
\end{aligned}
$$

and

$$
\mathbf{h}(x, y ; t)=\nu \nabla \cdot D^{\prime}(u) \nabla u .
$$

With periodic boundary conditions and an appropriate initial condition, note that the operator $L$ is uniformly parabolic in region $E_{T}=([0, L] \times[0, L]) \times$ $(0, T]$ for $u \in C^{4}\left([0, L]^{2},(0,1)\right)$. Under these and additional assumptions (regarding smoothness) and regularity arguments, it can be shown [54] that solutions $\sigma^{(2)}$ of Eq. 2.38 are $C^{2}\left(\mathcal{R}^{2}, \mathcal{R}\right)$ functions. Then bounds on $\sigma^{(2)}$ 
and up to its second derivatives exist. We will assume such bounds to obtain statements regarding numerical convergence of the lattice method.

(See and Lemma 2.48 of Section 2.4.2 for how the bounds apply to numerical consistency and Lemma 2.56 of Sestion 2.4 .3 for how they apply to continuum maximum and minimum principles for a truncated version of $n$, i.e., the truncated equilibrium expansion. ${ }^{28}$ )

- Solve for $\mathbf{n}^{(4)}$ : We find

$$
\begin{aligned}
\mathbf{n}^{(4)} & =\mathcal{L}^{+} \cdot \mathbf{g}^{(4)}+\sigma^{(4)} \mathbf{q}_{0} \\
& =\sum_{k=0}^{3} c_{k}^{(4)} \mathbf{q}_{k}
\end{aligned}
$$

where

$$
c_{0}^{(4)}=\sigma^{(4)}
$$

and

$$
c_{k}^{(4)}=\frac{1}{\lambda_{k}} \tilde{c}_{k}^{(4)}, k \in\{1,2,3\}
$$

$\mathcal{O}\left[\delta^{5}\right]$ : At this order, only the consistency condition is to be determined. This condition specifies $\sigma^{(3)}$. We will find that $\sigma^{(3)}(x, y ; t)=$ const. satisfies this condition. We find

$$
\begin{aligned}
\mathcal{L} \cdot \mathbf{n}^{(5)} & \\
= & {\left[\frac{L^{5}}{5 !}\left(\vec{e}_{k} \cdot \nabla\right)^{5} n_{k}^{(0)}+\frac{L T^{2}}{2}\left(\vec{e}_{k} \cdot \nabla\right) \frac{\partial^{2}}{\partial t^{2}} n_{k}^{(0)}+\frac{L^{3}}{6}\left(\vec{e}_{k} \cdot \nabla\right)^{3} \frac{\partial}{\partial t} n_{k}^{(0)}\right.} \\
& +\frac{L^{4}}{4 !}\left(\vec{e}_{k} \cdot \nabla\right)^{4} n_{k}^{(1)}+\frac{L^{2} T}{2}\left(\vec{e}_{k} \cdot \nabla\right)^{2} \frac{\partial}{\partial t} n_{k}^{(1)}+\frac{T^{2}}{2} \frac{\partial^{2}}{\partial t^{2}} n_{k}^{(1)} \\
& \left.+\frac{L^{3}}{3 !}\left(\vec{e}_{k} \cdot \nabla\right)^{3} n_{k}^{(2)}+L T\left(\vec{e}_{k} \cdot \nabla\right) \frac{\partial}{\partial t} n_{k}^{(2)}+\frac{L^{2}}{2 !}\left(\vec{e}_{k} \cdot \nabla\right)^{2} n_{k}^{(3)}+L\left(\vec{e}_{k} \cdot \nabla\right) n_{k}^{(4)}\right] \\
+ & (\text { Coll. })
\end{aligned}
$$

\footnotetext{
${ }^{28}$ This will be formally defined in the next section (see Definition 2.37 ).
} 


$$
=\sum_{k=0}^{3} \tilde{c}_{k}^{(5)} \mathbf{q}_{k}
$$

where (Coll.) is the contribution from the coefficient of $\delta^{5}$ in the collision operator expansion, Eq. 2.18. Since (Coll.) is orthogonal to the nullspace of the linearized collision operator, it does not contribute to the consistency condition. Note that $\tilde{c}_{0}^{(5)}$ is completely determined in view of this by the terms listed in Appendix A. The remaining coefficients, $\tilde{c}_{k}^{(5)}(k \in\{1,2,3\})$, are left uncalculated. ${ }^{29}$

- Consistency: Imposing the consistency condition that $q_{0} \cdot g^{(5)}=0$ implies $\tilde{c}_{0}^{(5)}=0$, or

$$
\sigma_{t}^{(3)}=\nu \nabla \cdot\left[D(u) \nabla \sigma^{(3)}+D^{\prime}(u) \sigma^{(3)} \nabla u\right]
$$

We see that $\sigma^{(3)}(x, y ; \hat{\imath})=$ const. satisfies the consistency condition. Then choosing $\sigma^{(3)}(x, y ; t)=0$ implies that $\tilde{c}_{1}^{(4)}=\tilde{c}_{2}^{(4)}=0$.

- Solve for $n^{(5)}$ : We would complete this calculation were we to desire the $\mathcal{O}\left[\delta^{6}\right]$ consistency condition, which would determine $\sigma^{(4)}$. But since $\sigma^{(4)}$ does not appear in the truncated equilibrium expansion, which is used in establishing consistency (as per the next section), it is not necessary to determine $\sigma^{(4)}$ nor, hence, $\mathbf{n}^{(5)}$.

We have shown how to apply the discrete Chapman.Enskog procedure to obtain the hydrodynamical equations, and higher order consistency conditions, associated with a lattice method.

\footnotetext{
${ }^{29}$ The $\tilde{c}_{k}^{(5)}(k \in\{1,2,3\})$ would need to be calculated for determining $\mathbf{n}^{(5)}$, which means also calculating the (Coll.) term. One would complete such calculations to determine the next order's consistency condition.
} 


\subsection{Convergence Analysis}

This section describes a numerical convergence theory for lattice Boltzmann methods; it applies to lattice gas methods to the extent that the Lattice Boltzmann Approximation is valid. Convergence is obtained here under the umbrella of monotone finite difference methods. (Appendix $\mathrm{C}$ contains a model convergence proof for a monotone finite difference scheme.) Three parts constitute proofs of convergence for monotone finite difference schemes: consistency, maximum and minimum principles, and stability (see, for example, [69]). This section develops these facets for lattice methods. In typical finite difference methods, one relates the computed and exact solutions. In lattice methods, the mean occupation numbers, $n$, take on the role of the "computed solution", while a truncated equilibrium expansion, h (see Definition 2.37), takes on the role of the "exact solution". We will be viewing $\mathrm{n}$ as an approximation to $\mathrm{h}$, i.e., the truncated equilibrium expansion is treated as an "exact" solution for purposes of demonstrating consistency, stability, and convergence of a lattice method. Then, generally, the terms related to convergence of lattice methods are described as follows: Consistency is obtained by applying the discrete Chapman-Enskog procedure to truncated equilibrium expansion and showing that the remainder (from Taylor's Theorem) tends to zero. Stability is defined in terms of boundedness of a linear operator that depends on both the mean occupation numbers, i.e., the solution to Eq. 2.8, and the truncated equilibrium expansion; the linear operator can be bounded in the $L_{1}$-norm with the support of discrete and continuum maximum and minimum principles. Conditions of monotonicity are used to establish the discrete maximum and minimum principles for the Lattice Boltzmann Equation, i.e., for the finite difference method, Eq. 2.8. The continuum maximum and minimum principles depend 
on maximum and minimum principles of the hydrodynamical equations of a lattice method and may depend on regularity of the higher order consistency conditions as revealed by the discrete Chapman-Enskog expansion. Finally, convergence is related to the difference between the hydrodynamical modes and the solutions of the hydrodynamical equations of a lattice method, and the norm of that difference in the limit as $\Delta t$ tends to zero. The general convergence result is that if

1. $\Delta x=L \delta$ and $\Delta t=T \delta^{2}$,

2. The collision operator is mass-conserving,

3. The initial condition, $\mathbf{n}_{\tilde{r}}^{0}$, is in the domain of monotonicity,

4. The collision operator is zero at the extrenne points of the domain of monotonicity,

5. The lattice method has a symmetric, nonpositive definite linearized collision uperator,

6. The hydrodynamical equations have maximum and minimum principles,

7. The higher order consistency conditions (up to $\mathcal{O}\left[\delta^{5}\right]$ ) have bounded solutions,

then the hydrodynamical modes of a lattice methed converge to the solution of its hydrodynamical equations. It is established that consistency and stability imply convergence. 


\subsubsection{Definitions}

The following terms and concepts are defined in this section: truncated equilibrium expansion, consistency, truncation error, conditions of monotonicity, domain of monotonicity, stability, and hydrodynamical errors, convergence. Generally, demonstrating consistency of a finite difference method for a partial differential equation entails showing that the truncation error, which depends on the difference operator applied to an exact solution, tends to zero in the limit as the grid spacing tends to zero. ${ }^{30}$ In lattice methods, the truncated equilibrium expansion takes on the role of the "exact" solution for consistency, stability, and convergence arguments. Stability is associated with a set of linear operators being bounded; the operators depend on both the computed and exact solutions. The domain of monotonicity is, loosely, the largest connected region in which the conditions of monotonicity ${ }^{31}$ are satisfied. Discrete maximum and minimum principles for a lattice method are established through arguments assuming that the conditions of monotonicity are satisfied (see Theorem 2.49). Convergence is then defined in terms of the "hydrodynamical errors" and their tendency (in some norm) toward zero as $\Delta t \rightarrow 0$. The hydrodynamical error is the difference between hydrodynamical modes and the solutions of the hydrodynamical equations. Consistency and stability can help establish convergence of a lattice method. The formal definitions of the terms related to convergence follow.

\footnotetext{
${ }^{30}$ See, for example, [66] or [70].

${ }^{31}$ The conditions of monotonicity are those conditions that, when satisfied, ensure that the lat tice method, seen as a difference operator, is monotonically increasing in all of its arguments. This is detailed in Definition 2.39.
} 
Definition 2.37 (Truncated Equilibrium Expansion). Let $\mathbf{n}=\sum_{j=0}^{\infty} \delta^{j} \mathbf{n}^{(j)}$ be the equilibrium expansion about equilibrium $\mathbf{n}^{(0)}$; where coefficients $\mathbf{n}^{(j)}$ are determined by the discrete Chapman-Enskog expansion. Then $\mathbf{h} \equiv \sum_{j=0}^{J} \delta^{j} \mathbf{h}^{(j)}$, where $\mathrm{h}^{(j)} \equiv \mathrm{n}^{(j)}, j \in\{0,1, \ldots, J\}$, is a truncated equilibrium expansion of order $J$, where $J$ is a (finite) positive integer.

DEFINITION 2.38 (CONSISTENCY). Let equilibrium expansion

$$
\mathbf{n}_{\mathfrak{i}}^{n}=\left(\mathbf{n}^{(0)}\right)_{i}^{n}+\delta\left(\mathbf{n}^{(1)}\right)_{i}^{n}+\delta^{2}\left(\mathbf{n}^{(2)}\right)_{i}^{n}+\cdots
$$

satisfy the Lattice Boltzmann Equation, Eq. 2.8, where the $\mathbf{n}^{(j)}$ are determined by the discrete Chapman-Enskog procedure of Section 2.3. Let $\mathbf{h}_{\mathfrak{i}}^{n}$ be a truncated equilibrium expansion, i.e.,

$$
\mathbf{h}_{\mathfrak{i}}^{n}=\sum_{j=0}^{J} \delta^{j}\left(\mathbf{h}^{(j)}\right)_{i}^{n},
$$

of order $J$, in which $\mathbf{h}^{(j)} \equiv \mathbf{n}^{(j)}$, and

$$
\mathcal{A} \mathbf{h}_{\mathfrak{i}}^{n}=\mathbf{h}_{\mathfrak{i}}^{n}+\mathcal{C}\left(\mathbf{h}_{\mathfrak{i}}^{n}\right)-\mathcal{T}\left(\mathbf{h}_{\mathfrak{i}}^{n}\right),
$$

where truncation error, $\mathcal{T}\left(\mathbf{h}_{\mathfrak{f}}^{n}\right)$, is determined from the Taylor series expansion with remainder in the Chapman-Enskog procedure on $\mathrm{h}_{\mathfrak{i}}^{n}$. Suppose for some norm, $\|\cdot\|$,

$$
\lim _{\Delta t \rightarrow 0} \frac{1}{\Delta t}\|r(\mathbf{h})\|=0, \quad 0 \leq t \leq T
$$

for all sufficiently smooth ${ }^{32}$ occupation functions h of Eq. 2.40.

DEFInition 2.39 (Conditions of Mono'Tonicity). Let a lattice method be given that satisfies the Lattice Boltzmann Equation, Eq. 2.8. Define

$$
H \equiv\left[H_{k}\left(\mathbf{n}_{\vec{\imath}-\vec{e}_{k}}^{n}\right)\right]_{k=0}^{d-1},
$$

\footnotetext{
32 "Sufficiently smooth" means h has en ough continuous spatial and temporal derivatives so that. Taylor's Theorem applies. (See, for example, [55, Section 6.8 (Theorem 10)] for a statement the theorem.)
} 
where

$$
\left(n_{k}\right)_{\vec{i}}^{n+1}=H_{k}^{\prime}\left(\mathbf{n}_{\vec{i}-\vec{e}_{k}}^{n}\right)=\left(n_{k}\right)_{\vec{i}-\vec{e}_{k}}^{n}+\mathcal{C}_{k}\left(\mathbf{n}_{\vec{i}-\vec{e}_{k}}^{n}\right) .
$$

Consider $H$ as a vector function of its arguments. Then let

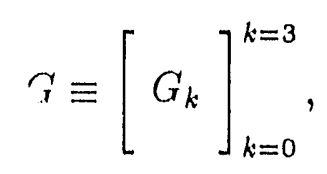

in which each $G_{k}$ is the gradient of $H_{k}$, i.e.,

$$
G_{k} \equiv \frac{\partial}{\partial \mathbf{n}_{\tilde{i}-\vec{e}_{k}}^{n}} H_{k}\left(\mathbf{n}_{\mathfrak{i}_{-}-\vec{e}_{k}}^{n}\right) .
$$

Then the inequalities,

$$
G_{k} \geq 0 \quad \forall k \in\{0,1, \ldots, d-1\}
$$

are called tire conditions of monotonicity of a lattice method.

The domain of monotonicity, denoted $\mathcal{E}$, is a $d$-dimensional rectangle in which the conditions of monotonicity are satisfied. The domain of monotonicity cannot be extended in a connected fashion to another region, the union in which the conditions of monotonicity are satisfied.

Definition 2.40 (Domain of Monotonicity). Let a lattice method be given that satisfies the Lattice Boltzmann Equation, Eq. 2.8. Let $M_{-}^{(k)}$ and $M_{+}^{(k)}$ be such that $\mathcal{E}^{(k)} \equiv\left[M_{-}^{(k)}, M_{+}^{(k)}\right] \subset[0,1] \quad \forall k \in\{0,1, \ldots, d-1\}$. Let

$$
\mathbf{M}_{-} \equiv\left[M_{-}^{(k)}\right]_{k=0}^{d-1} \text { and } \mathbf{M}_{+} \equiv\left[M_{+}^{(k)}\right]_{k=0}^{d-1} \text {. }
$$

And let $\mathcal{E} \equiv \mathcal{E}^{(0)} \times \mathcal{E}^{(1)} \times \cdots \times \mathcal{E}^{(d-1)} \subset[0,1]^{d}$. Suppose $\mathcal{E}$ defines a region in which the conditions of monotonicity are satisfied. Suppose that there does not ex ist 
some direction $\hat{k}$ for which the $\mathcal{E}^{(\hat{k})}$ can be extended and still satisfy the conditions of monotonicity in the respectively extended $\mathcal{E}$ (where the extended $\mathcal{E}$ is a connected region). Furthermore, suppose that $\mathcal{E}$ has volume, i.e., $v(\mathcal{E})>0$. Then $\mathcal{E}$ is called the domain of monotonicity, and $\mathrm{M}_{+}$and $\mathrm{M}_{+}$are called the extreme points of $\mathcal{E}$.

Definition $2.41\left(L\left[\mathbf{n}^{n}, \mathrm{~h}^{n}\right]\right)$. Let $\mathbf{n}^{n}$ satisfy Eq. 2.8. Let $\mathrm{h}^{n}$ be a truncated equilibrium expansion of order $J$ that satisfies Eq. 2.10. Define the error $\mathrm{e}^{n}$ by

$$
\mathrm{e}^{n} \equiv \mathrm{n}^{n}-\mathrm{h}^{n}
$$

Then operator $L\left[\mathrm{n}^{\mathrm{n}}, \mathrm{h}^{\mathrm{n}}\right]$ is defined by

$$
L\left[\mathbf{n}^{n}, \mathbf{h}^{n}\right]=\int_{0}^{1}\left[I+\mathcal{D C}\left(\mathbf{n}^{n} \dot{r} s\left(\mathbf{h}^{n}-\mathbf{n}^{n}\right)\right)\right] d s,
$$

so that by the Fundamental Theorem of Calculus

$$
\mathcal{A} \mathbf{e}^{n}=\mathbf{e}^{n}+\mathcal{C}\left(\mathbf{e}^{n}\right)+\mathcal{T}\left(\mathbf{h}^{n}\right)=L\left[\mathbf{n}^{n}, \mathbf{h}^{n}\right] \mathbf{e}^{n}+\mathcal{T}\left(\mathbf{h}^{n}\right)
$$

Definition 2.42 (Stability). Let $\mathbf{n}_{\tilde{i}}^{n}, \mathrm{~h}_{\tilde{i}}^{n}$, and $L\left[\mathbf{n}^{n}, \mathrm{~h}^{n}\right]$ be as in Definition 2.41. Then a lattice method is said to be stable up to time $T$ if for some $\tau>0$, the infinite set of operators,

$$
\left\{\prod_{m=0}^{n} L\left[\mathbf{n}^{m}, \mathrm{~h}^{m}\right] \mid 0<\Delta t<\tau \text { and } 0 \leq n \Delta t \leq T\right\}
$$

is uniformly bounded.

Definition 2.43 (Hydrodynamical Mode). Let $\mathbf{n}_{\mathfrak{i}}^{n}$ salisfy the Lattice Boltzmann Equation, Eq. 2.8. Then let $p_{i}^{n}=\left[\left(p_{k}\right)_{i}^{n}\right]_{k=0}^{d-1}$, where

$$
\left(p_{k}\right)_{\mathfrak{i}}^{n} \equiv \frac{\mathbf{q}_{k} \cdot \mathbf{n}_{\mathfrak{r}}^{n}}{d} \quad \forall k \in\{0,1, \ldots, d-1\} \text {. }
$$


Then $p_{k}$ such that $\mathrm{q}_{k} \in$ nullspace $(\mathcal{L})$ is called a hydrodynamical mode of the lattice method.

Definition 2.44 (HYDRODYNAMICAL ERror). Let $\mathrm{n}_{\mathfrak{i}}^{n}$ and $\mathrm{h}_{\tilde{t}}^{n}$ be as in Definition 2.4 . Let $\rho_{i}^{n}=\left[\left(\rho_{k}\right)_{i}^{n}\right]_{k=0}^{d-1}$, where

$$
\left(\rho_{k}\right)_{\hat{i}}^{n} \equiv \frac{\mathrm{q}_{k} \cdot \mathbf{h}_{i}^{n}}{d} \forall k \in\{0,1, \ldots, d-1\}
$$

Then $\rho_{k}$ such that $\mathbf{q}_{k} \in$ nullspace $(\mathcal{L})$ is called a discretized hydrodynamical mode of the truncated equilibrium expansion. Let $\mathfrak{f}_{\mathfrak{i}}^{n} \equiv\left[\left(f_{k}\right)_{i}^{n}\right]_{k=0}^{d-1}$, where

$$
\left(f_{k}\right)_{i}^{n} \equiv\left(p_{k}\right)_{i}^{n}-\left(\rho_{k}\right)_{i}^{n} \forall k \in\{0,1, \ldots, d-1\} \text {. }
$$

Then $f_{k}$ such that $\mathbf{q}_{k} \in$ nullspace $(\mathcal{L})$ is called a hydrodynamical error of the lattice method. Let $F_{k}^{n} \equiv\left[\left(f_{k}\right)_{\mathfrak{i}}^{n}\right]_{\mathfrak{i} \in \mathfrak{L}}$ and $F^{n} \equiv\left[F_{k}^{n}\right]_{k=0}^{d-1}$.

LEMMA 2.45. $\mathrm{f}_{\vec{i}}^{n}=\left[\frac{\mathrm{q}_{k} \cdot \mathrm{e}_{i}^{n}}{d}\right]_{k=0}^{d-1}$, i.e., $\mathrm{f}_{i}^{n}=\frac{1}{d} \mathrm{Q} \cdot \mathbf{e}_{i}^{n}$.

Proof. This is a combined result of Definitions 2.33, 2.41, 2.43, and 2.44.

Definition 2.46 (Convergence). If $\forall k$ such that $\mathbf{q}_{k} \in \operatorname{nullspace}(\mathcal{L})$, $\lim _{\Delta t \rightarrow 0} \frac{1}{\Delta t}\left\|F_{k}^{n}\right\|=0 \forall n$ such that $0 \leq n \Delta t \leq T$ in some norm, $\|\cdot\|$, we say that the hydrodynamical modes of a lattice method converges to the solution of its consistency conditions, as determined in the discrete Chapman-Enskog expansion.

\subsubsection{Consistency}

In a general sense, consistency of lattice methods comes from applying the discrete Chapman-Enskog expansion of Section 2.3 to n. That expansion imposes consistency conditions, and those conditions lead to overall consistency of the method. In that 
process, $\mathbf{n}$ is assumed to be an infinite expansion about an equilibrium, i.e.,

$$
\mathbf{n}=\sum_{j=0}^{\infty} \delta^{j} \mathbf{n}^{(j)}
$$

where the $\mathbf{n}^{(j)}$ are determined at each step of matching the expansions (see Section 2.3.5). Formal consistancy arguments involve applying the discrete Chapman-Enskog expansion to a truncated version of $\mathbf{n}$, which is denoted $\mathrm{h}$ (see Definition 2.37). The truncation order depends on characteristics of the lattice method, such as inclusion of $\mathcal{O}[\delta]$ advection and, though not yet verified, probably the number of directions, $d$, and spatial dimensions, $p$. The application of the discrete Chapman-Enskog procedure (on $\mathrm{n}$ ) determines the $\sigma^{(j)}$ paraineters (each set of $\sigma^{(j)}$ parameters is introduced by the nullspace of the linearized collision operator). Any $\sigma^{(j)}$ parameters appearing in the truncated expansion, h, satisfy (by definition) the consistency conditions determined by the discrete Chapman-Enskog exnansion on $\mathbf{n}$.

THEOREM 2.47 (CONSISTENCY). Let $\Delta x=L \delta$ and $\Delta t=T \delta^{2}$ for spatial and temporal scale lengths $L$ and $T$, respectively. Let $\mathrm{n}=\sum_{j=0}^{\infty} \delta^{j} \mathbf{n}^{(j)}$ satisfy ine Lattice Boltzmann Equation, Eq. 2.8, where $\mathrm{n}^{(0)}$ is an equilibrium and the remaining $\mathrm{n}^{(j)}$ are determined by the discrete Chapman-Enskog procedure of Section 2.3. Let $\mathrm{h}$ be an associated truncated equilibrium expansion of order $J$ for some $J \in \mathcal{J}$ and $J \geq$ 1 so that $\mathrm{h}=\sum_{j=0}^{J} \delta^{j} \mathrm{~h}^{(j)}$. Suppose that $\left\{\mathrm{h}^{(j)} \mid j \in\{0,1, \ldots, J\}, 0 \leq n \Delta t \leq T\right\}$ is uniformly bounded. Then h satisfies Eq. 2.40, in which $\mathcal{T}(\mathrm{h})=\mathcal{O}\left[\delta^{J+1+K}\right]$ for some integer $K \geq 0$. Further, if $J+1+K>2$, then

$$
\lim _{\Delta t \rightarrow 0} \frac{1}{\Delta t}\|\mathcal{T}(\mathrm{h})\|=0
$$

for some norm, $\|\cdot\|$. 
Proof. Note that we may write

$$
\mathcal{T}(\mathbf{h})=\sum_{j=0}^{J} \delta^{j} \mathbf{T}^{(j)}+\mathcal{O}\left[\delta^{J+1}\right]
$$

for some coefficients $\mathbf{T}^{(j)}, j \in\{0,1, \ldots, J\}$. To prove the first part of the theorem, it is enough to show that $\mathbf{T}^{(j)}=\mathbf{0} \forall j \in\{0,1, \ldots, J\}$. Since $\mathbf{h}^{(0)}=\mathbf{n}^{(0)}$ is an equilibrium solution, $\mathcal{C}\left(\mathbf{h}^{(0)}\right)=0$; hence, $\mathbf{T}^{(0)}=0$. Aid since $\mathbf{h}^{(j)}=\mathbf{n}^{(j)} \forall j \in\{1,2, \ldots, J\}$, $\mathcal{A} \mathrm{h}-\mathrm{h}-\mathcal{C}(\mathbf{h})=\mathcal{O}\left[\delta^{J+1}\right]$ hy appli zation of the discrete Chapman-Enskog procedure on $\mathbf{n}$, i.e., the $\mathbf{n}^{(j)}$ are determined in a way to insure this. Hence, $\exists K \geq 0$ such that $\mathcal{T}(\mathbf{h})=\mathcal{O}\left[\delta^{J+1+K}\right]$.

To prove the second part, we assume that $J+1+K>2$. Then $\tau^{\prime}(\mathbf{h})=\mathcal{O}\left[\delta^{J+1+K}\right]$, and $\exists$ a constant $C$ such that $\|T(h)\| \leq C \delta^{J+1+K}$ for some norm, $\|\cdot\|$. Finally,

$$
\begin{aligned}
\frac{1}{\Delta t}\|\mathcal{T}(\mathrm{h})\| & \leq \frac{1}{T \delta^{2}} C \delta^{J+1+K} \\
& \leq \frac{C}{T} \delta^{J+1+K-2} \\
& \rightarrow 0 \text { as } \measuredangle t=\frac{T}{L^{2}} \delta^{2} \rightarrow 0
\end{aligned}
$$

since $J+1+K-2>0$.

Example ( $L B_{1}$ : Consistency).

Lemma 2.48 (CONSISTENCY OF $\mathrm{LB}_{1}$ ). Let $\mathbf{n}=\sum_{j=0}^{\infty} \delta^{j} \mathbf{n}^{(j)}$ be determined by the discrete Chapman-Enskog procedure. Let the iruncated equilibrium expansion, h, ?: aefined by

$$
\mathbf{h} \equiv \sum_{j=0}^{3} \delta^{j} \mathbf{h}^{(j)}
$$

where $\mathbf{h}^{(j)} \equiv \mathbf{n}^{(j)}$ for $j \in\{0,1,2,3\}$, where the $\mathbf{n}^{(j)}, j \in\{0,1,2,3\}$, ore determined by $E_{1}$ s. 2.27, 2.28, 2.30, and 2.32. Then h satisfies Eq. 2.40; in which 
$\mathcal{T}(\mathbf{h})=\mathcal{O}\left[\delta^{4}\right]$. Suppose that $\sigma^{(0)} \in C^{4}\left([0, L]^{2},(0,1)\right)$ and $\sigma^{(2)} \in C^{2}\left(\mathcal{R}^{2}, \mathcal{R}\right){ }^{34}$ Let $\Delta t=T \delta^{2}, \Delta x=L \delta$, and $\nu=L^{2} /\left(2 T^{\prime}\right)$, for spatial and temporal scale lengths $L$ and $T$, respectively. Then

$$
\lim _{\Delta t \rightarrow 0} \frac{1}{\Delta t}\|\mathcal{T}(\mathbf{h})\|=0
$$

for some norm, $\|\cdot\|$.

Proof. First, we determine the parameters of the truncated equilibrium expansion in terms of the expansion for $\mathbf{n}$. Then we determine the remainder, $\mathcal{T}(\mathbf{h})$. Cirially, we show that $\lim _{\Delta t \rightarrow 0} \frac{1}{\Delta t}\|\mathcal{T}(\mathbf{h})\|=0$.

\section{Part 1: Determining the Truncated Equilibrium Expansion}

Recall that any constant $\sigma^{(1)}(x, y ; t)$ and ${ }^{(3)}(x, y ; t)$ satisfy the $\mathcal{O}\left[\delta^{3}\right]$ and $\mathcal{O}\left[\delta^{5}\right]$ consistency conditions, i.e., Eq. 2.31 and Eq. 2.39, respectively. Choose $\sigma^{(1)}(x, y ; t)$ $=\sigma^{(3)}(x, y ; t)=0$. Then using that $\mathbf{h}^{(j)} \equiv \mathbf{n}^{(j)}$ and Eqs. 2.27, 2.28, 2.30, and 2.32 for $\mathbf{n}^{(0)}, \mathbf{n}^{(1)}, \mathbf{n}^{(2)}$, and $\mathbf{n}^{(3)}$, respectively, we find

$$
\begin{gathered}
\mathbf{h}^{(0)}=\mathbf{q}_{0} u . \\
\mathbf{h}^{(1)}=\sum_{k=0}^{3} c_{k}^{(1)} \mathbf{q}_{k},
\end{gathered}
$$

where

$$
\begin{aligned}
c_{0}^{(1)} & =0, \\
c_{1}^{(1)} & =\frac{L}{\lambda} u_{x}, \\
c_{2}^{(1)} & =\frac{L}{\lambda} u_{y}, \\
c_{3}^{(1)} & =0 ;
\end{aligned}
$$

\footnotetext{
${ }^{34}$ Recall that $u(x, y ; t) \equiv v^{(0)}(x, y ; t)$ and that the $\sigma^{(j)}$ parameters are determined by applying the discrete Chapisan-Enskog procedure to n. See Eqs. 2.29 and 2.37 for $\sigma^{(0)}$ and $\sigma^{(2)}$, respectively.
} 


$$
\mathbf{h}^{(2)}=\sum_{k=0}^{3} c_{k}^{(2)} \mathbf{q}_{k},
$$

where

$$
\begin{aligned}
c_{0}^{(2)} & =\sigma^{(2)} \\
c_{1}^{(2)} & =0 \\
c_{2}^{(2)} & =0 \\
c_{3}^{(2)} & =\frac{L^{2}}{2 \lambda}\left[\frac{1}{2} D^{\prime}(u)\left(\left(u_{x}\right)^{2}-\left(u_{y}\right)^{2}\right)-\frac{1}{2}\left(\frac{\partial}{\partial x}\left(D(u) u_{x}\right)-\frac{\partial}{\partial y}\left(D^{\prime}(u) u_{y}\right)\right)\right]
\end{aligned}
$$

and

$$
\mathbf{h}^{(3)}=\sum_{k=0}^{3} c_{k}^{(3)} \mathbf{q}_{k},
$$

where

$$
\begin{aligned}
c_{0}^{(3)} & =0, \\
c_{1}^{(3)} & =\frac{1}{\lambda}\left[\frac{L^{3}}{6} u_{x x x}+L T u_{x t}+\left(\frac{L^{3}}{2} \frac{\partial^{2}}{\partial x^{2}}+L T \frac{\partial}{\partial t}\right)\left(\frac{1}{\lambda} u_{x}\right)\right. \\
& +\frac{L^{3}}{2} \frac{\partial}{\partial x}\left[\frac{1}{2 \lambda} D^{\prime}(u)\left(\left(u_{x}\right)^{2}-\left(u_{y}\right)^{2}\right)-\frac{1}{\lambda}\left(\frac{\partial}{\partial x},-\frac{\partial}{\partial y}\right) \cdot D(u) \nabla u\right] \\
& +L \sigma_{x}^{(2)}+\frac{4 L^{3}}{\lambda^{3}}\left(u_{x}\right)\left(u_{y}\right)^{2}-L D^{\prime}(u) \lambda \sigma^{(2)} u_{x} \\
& \left.+\frac{L^{3}}{4} D^{\prime}(u) u_{x}\left[D^{\prime}(u)\left(\left(u_{x}\right)^{2}-\left(u_{y}\right)^{2}\right)-2\left(\frac{\partial}{\partial x},-\frac{\partial}{\partial y}\right) \cdot D(u) \nabla u\right]\right], \\
c_{2}^{(3)} & =\frac{1}{\lambda}\left[\frac{L^{3}}{6} u_{y y y}+L T u_{y t}+\left(\frac{L^{3}}{2} \frac{\partial^{2}}{\partial y^{2}}+L T \frac{\partial}{\partial t}\right)\left(\frac{1}{\lambda} u_{y}\right)\right. \\
& -\frac{L^{3}}{2} \frac{\partial}{\partial y}\left[\frac{1}{2 \lambda} D^{\prime}(u)\left(\left(u_{x}\right)^{2}-\left(u_{y}\right)^{2}\right)-\frac{1}{\lambda}\left(\frac{\partial}{\partial x},-\frac{\partial}{\partial y}\right) \cdot D(u) \nabla u\right] \\
& +L \sigma_{y}^{(2)}+\frac{4 L^{3}}{\lambda^{3}}\left(u_{x}\right)^{2}\left(u_{y}\right)-L D^{\prime}(u) \lambda \sigma^{(2)} u_{y} \\
& \left.-\frac{L^{3}}{4} D^{\prime}(u) u_{y}\left[D^{\prime}(u)\left(\left(u_{x}\right)^{2}-\left(u_{y}\right)^{2}\right)-2\left(\frac{\partial}{\partial x},-\frac{\partial}{\partial y}\right) \cdot D(u) \nabla u\right]\right], \\
c_{3}^{(3)} & =0 .
\end{aligned}
$$


Part 2: Determining the Remainder

Now, to determine the truncation error, i.e., the remainder, $\mathcal{T}(\mathbf{h})$, let us apply the discrete Chapman-Enskog expansion to $h$.

We carefully apply the advection and collision operator expansions (Eq. 2.24 and Eq. 2.18, respectively) to the truncated equilibrium expansion, h. Care is taken in noting that the collision operator is $\mathcal{O}[1]$ and that $\mathrm{h}$ contains only up to the $\mathcal{O}\left[\delta^{3}\right]$ terms of the discrete Chapman-Enskog expansion. Thus, some of the terms in the referenced expansions do not appear in the expansions with $h$. The resulfing expansion follows:

$$
\mathcal{A} \mathbf{h}-\mathbf{h}-\mathcal{C}(\mathbf{h})=-\mathcal{T}(\mathbf{h})=-\sum_{j=0}^{4} \mathbf{T}^{(j)} \delta^{j}+\mathcal{O}\left[\delta^{5}\right]
$$

where

$$
\begin{aligned}
\mathbf{T}^{(0)}= & \mathcal{C}\left(\mathbf{h}^{(0)}\right) \\
\mathbf{T}^{(1)}= & \mathcal{L} \cdot \mathbf{h}^{(1)}-\left[L\left(\vec{e}_{k} \cdot \nabla\right) h_{k}^{(0)}\right] \\
\mathbf{T}^{(2)}= & \mathcal{L} \cdot \mathbf{h}^{(2)}+\frac{1}{2} \mathcal{D}^{2} \mathcal{C}\left(\mathbf{h}^{(0)}\right) \cdot \mathbf{h}^{(1)} \mathbf{h}^{(1)} \\
- & {\left[\frac{L^{2}}{2 !}\left(\vec{e}_{k} \cdot \nabla\right)^{2} h_{k}^{(0)}+T \frac{\partial}{\partial t} h_{k}^{(0)}+L\left(\vec{e}_{k} \cdot \nabla\right) h_{k}^{(1)}\right] } \\
\mathbf{T}^{(3)}= & \mathcal{L} \cdot \mathbf{h}^{(3)}+\frac{1}{6} \mathcal{D}^{3} \mathcal{C}\left(\mathbf{h}^{(0)}\right) \cdot \mathbf{h}^{(1)} \mathbf{h}^{(1)} \mathbf{h}^{(1)}+\mathcal{D}^{2} \mathcal{C}\left(\mathbf{h}^{(0)}\right) \cdot \mathbf{h}^{(1)} \mathbf{h}^{(2)} \\
- & {\left[\frac{L^{3}}{3 !}\left(\vec{e}_{k} \cdot \nabla\right)^{3} h_{k}^{(0)}+L T\left(\vec{e}_{k} \cdot \nabla\right) \frac{\partial}{\partial t} h_{k}^{(0)}+\frac{L^{2}}{2 !}\left(\vec{e}_{k} \cdot \nabla\right)^{2} h_{k}^{(1)}\right.} \\
& \left.+T \frac{\partial}{\partial t} h_{k}^{(1)}+L\left(\vec{e}_{k} \cdot \nabla\right) n_{k}^{(2)}\right], \\
\mathbf{T}^{(4)}= & \frac{1}{24} \mathcal{D}^{4} \mathcal{C}\left(\mathbf{h}^{(0)}\right) \cdot \mathbf{h}^{(1)} \mathbf{h}^{(1)} \mathbf{h}^{(1)} \mathbf{h}^{(1)}+\frac{1}{2} \mathcal{D}^{3} \mathcal{C}\left(\mathbf{h}^{(0)}\right) \cdot \mathbf{h}^{(1)} \mathbf{h}^{(1)} \mathbf{h}^{(2)} \\
+ & \frac{1}{2} \mathcal{D}^{2} \mathcal{C}\left(\mathbf{h}^{(0)}\right) \cdot \mathbf{h}^{(2)} \mathbf{h}^{(2)}+\mathcal{D}^{2} \mathcal{C}\left(\mathbf{h}^{(0)}\right) \cdot \mathbf{h}^{(1)} \mathbf{h}^{(3)} \\
- & {\left[\frac{L^{4}}{4 !}\left(\vec{e}_{k} \cdot \nabla\right)^{4} h_{k}^{(0)}+\frac{L^{2} T}{2}\left(\vec{e}_{k} \cdot \nabla\right)^{2} \frac{\partial}{\partial t} h_{k}^{(0)}+\frac{T^{2}}{2} \frac{\partial^{2}}{\partial t^{2}} h_{k}^{(0)}+L\left(\vec{e}_{k} \cdot \nabla\right) h_{k}^{(3)}\right.}
\end{aligned}
$$




$$
\left.+\frac{L^{2}}{2 !}\left(\vec{e}_{k} \cdot \nabla\right)^{2} h_{k}^{(2)}+T \frac{\partial}{\partial t} h_{k}^{(2)}+\frac{L^{3}}{3 !}\left(\vec{e}_{k} \cdot \nabla\right)^{3} h_{k}^{(1)}+L T\left(\vec{e}_{k} \cdot \nabla\right) \frac{\partial}{\partial t} h_{k}^{(1)}\right] .
$$

Now, we derive explicit expressions for the coefficients $T^{(j)}$ as linear combinations of the eigenvectors of the linearized collision operator. We will write

$$
\mathbf{T}^{(j)}=\sum_{k=0}^{3} \mathbf{T}_{k}^{(j)} \mathbf{q}_{k}
$$

where $T_{k}^{(j)}$ is a scalar expression. With this notation we may write the Taylor remainder as follows:

$$
T_{k}=\sum_{j=0}^{4} \delta^{j} \sum_{l=0}^{l=3} \mathrm{~T}_{l}^{(j)} \mathbf{q}_{l, k} .
$$

We find by the discrete Chapman-Enskog expansion on $n$ that

$$
\mathbf{T}^{(j)}=\mathbf{0}, \quad j \in\{0,1,2,3\}
$$

and

$$
\mathrm{T}^{(4)}=\sum_{k=0}^{3} \mathrm{~T}_{k}^{(4)} \mathbf{q}_{k}
$$

where

$$
\begin{aligned}
& \mathbf{T}_{0}^{(4)}=-\tilde{c}_{0}^{(4)}=0,\left(\text { because of the } \mathcal{O}\left[\delta^{3}\right]\right. \text { consistency condition) } \\
& \mathbf{T}_{1}^{(4)}=-\tilde{c}_{1}^{(4)}=0,\left(\text { because } \sigma^{(3)}=0\right) \\
& \mathbf{T}_{2}^{(4)}=-\tilde{c}_{2}^{(4)}=0,\left(\text { because } \sigma^{(3)}=0\right) \\
& \mathbf{T}_{3}^{(4)}=-\left.\tilde{c}_{3}^{(4)}\right|_{\sigma^{(1)}(x, y ; t)=\sigma^{(3)}(x, y ; t)=0}
\end{aligned}
$$

in which the $\tilde{c}_{k}^{(4)}$ are given in Eqs. 2.33-2.36. Note that $\mathrm{T}_{3}^{(4)}$ is a function of $u$ and $\sigma^{(2)}$ and up to their fourth spatial derivatives (using the $\mathcal{O}\left[\delta^{2}\right]$ and $\mathcal{O}\left[\delta^{3}\right]$ consistency conditions to remove temporal derivatives.)

We have determined that the remainder, $\mathcal{T}(\mathbf{h})$, is $\mathcal{O}\left[\delta^{4}\right]$. (Note that it in $\operatorname{span}\left(q_{3}\right)$. This is the least accurate mode; it drives the truncation error.) 


\section{Part 3: Showing Consistency}

The combined results of parts 1 and 2 establish consistency of $L B_{1}$, as summarized by the following: Note that $\mathbf{h}^{(j)}, j \in\{0,1,2,3\}$, are uniformly bounded since

$$
\sigma^{(0)} \in C^{4}\left([0,1]^{2},(0,1)\right) \text { and } \sigma^{(2)} \in C^{2}\left(\mathcal{R}^{2}, \mathcal{R}\right)
$$

and they satisfy the $\mathcal{O}\left[\delta^{2}\right]$ and $\mathcal{O}\left[\delta^{4}\right]$ consistency conditions, Eq. 2.29 and Eq. 2.37, respectively. Hence, $\exists$ a norm, $\|\cdot\|$, such that

$$
\lim _{\Delta t \rightarrow 0} \frac{1}{\Delta t}\|\mathcal{T}(\mathbf{h})\|=0
$$

by Theorem 2.47 .

\subsubsection{Maximum and Minimum Principles}

The crux of the upcoming convergence argument relies on maximum and minirnum principles for the finite difference equation, i.e., the Lattice Boltzmann Equation, Eq. 2.8, and for the dependent variables appearing in the truncated equilibrium expansion. The former involves monotonicity properties of the difference equation while the latter involves maximum and minirnum principles for partial differential equations. (These amount to obtaining discrete and continuum maximum/minimum principles.) The combined maximum/minimum principle results (along with certain regularity conditions) will provide sufficient innditions to guarantee stability of a lattice method.

The discrete maximum/minimum principle depends on monotonicity of the finite difference method, i.e., that the r.h.s. of the Lattice Boltzmann Equation, Eq. 2.8, is monotonically increasing as a function of its arguments. Consider the difference 
nethod, 35

$$
U_{j}^{n+1}=U_{j}^{n}-\frac{\Delta t}{4 \Delta x}\left[\left(U_{j+1}^{n}\right)^{2}-\left(U_{j-1}^{n}\right)^{2}\right]+\frac{\nu \Delta t}{(\Delta x)^{2}}\left[U_{j+1}^{n}-2 U_{j}^{n}+U_{j-1}^{n}\right]
$$

for the one-dimensional viscuus Burgers equation,

$$
u_{t}+u u_{x}=u^{\prime \prime} u_{x x}
$$

Note that the r.h.s, of Eq. 2.49 can be written as a function $H\left(U_{j-1}^{n}, U_{j}^{n}, U_{j+1}^{n}\right)$. Then the difference method, Eq. 2.49, is a monotone finite difference method if $H$ is monotonically increasing in all of its arguments $\left[69, \mathrm{Ch}\right.$. IV]. We assume $H \in C^{1}\left(\mathcal{R}^{3}, \mathcal{R}\right)$. Thus, the gradient of $H$ may be used to determine whether $H$ is monotonically increasing; a nonnegative gradient yields the desired result. It may be the case that some conditions on the arguments of $H^{r}$ (in addition to the familiar stability criterion) must be imposed to guarantee that the difference method is monotone. These are called the conditions of monotonicity. By the nature of the recursion relation in which $H$ appears, the monotonicity conditions are restrictions on the initial conditions, i.e., $U_{j}^{0}$ for all grid locations $j$. (See Appendix $\mathrm{C}$ for the details of this example.)

A similar function, $H$, applies in lattice methods. However, $H$ turns out to be a vector of " $H$ " functions (each of which is denoted $H_{k}$ ). In this case, we say that $H$ is monotonically increasing if each $H_{k}$ is monotonically increasing separately in all arguments (see Definition 2.39. An important contribution of this work is that a lattice method may only be conditionally monotone. That is, it may be a monotone difference method for only certain initial conditions, i.e., ones meeting the conditions of monotonicity. The conditions of monotonicity form a basis of a discrete maximum/minimum principle for lattice methods. Upon these conditions rests the crux

\footnotetext{
${ }^{35}$ This is the example discussed in Appendix C.
} 
of the convergence proofs herein; hence, it may be that a lattice method converges for orly initial conditions meeting the conditions of monotonicity.

Let $\mathcal{E}$ be the domain of monotonicity of a lattice method (see Definition 2.40). Let $\mathrm{n}_{\mathfrak{f}}^{n}$ satisfy the Lattice Boltzmann Equation, Eq. 2.8, and let truncated equilibrium expansion $h_{\mathfrak{r}}^{n}$ satisfy the lattice Boltzmann equation with remainder, Eq. 2.40. Then the discrete and continuum maximum/minimum principles we desire to establish are:

1. If $\left(\forall \vec{\imath} \in \mathfrak{L}, \mathbf{n}_{\mathfrak{\imath}}^{0} \in \mathcal{E}\right)$, then $\left(\mathbf{n}_{\mathfrak{r}}^{n} \in \mathcal{E}, \forall \vec{\imath}, \forall n\right)$.

2. If $\left(\forall \vec{\imath} \in \mathfrak{L}, h_{\mathfrak{i}}^{0} \in \mathcal{E}\right)$, then $\left(h_{\mathfrak{i}}^{\mathfrak{n}} \in \mathcal{E}, \forall \vec{\imath}, \forall n\right)$.

\section{Discrete Maximum/Minimum Principle}

Theorem 2.49 (Discrete Maximum/Minimum Principle). Let the domain of monotonicity, $\mathcal{E}=\mathcal{E}^{(0)} \times \mathcal{E}^{(1)} \times \cdots \times \mathcal{E}^{(d-1)} \subset[0,1]^{d}$, for a lattice method be given, where $\mathcal{E}^{(k)}=\left[M_{-}^{(k)}, M_{+}^{(k)}\right]$. Let

$$
\mathbf{M}_{-}=\left[M_{-}^{(k)}\right]_{k=0}^{d-1} \text { and } \mathbf{M}_{+}=\left[M_{+}^{(k)}\right]_{k=0}^{d-1}
$$

be the extreme points of the domain of monotonicity. Suppose that the initial condition is in the domain of monotonicity, i.e., $\mathrm{n}_{\mathfrak{r}}^{0} \in \mathcal{E}, \forall \vec{\imath} \in \mathcal{L}$. Further suppose that $\mathcal{C}\left(\mathbf{M}_{-}\right)=\mathcal{C}\left(\mathbf{M}_{+}\right)=0 \in[0,1]^{d}$. Then for all time steps $n, \mathbf{n}_{\mathfrak{r}}^{n} \in \mathcal{E}, \forall \vec{\imath} \in \mathfrak{L}$.

We will prove the theorem by induction on $n$ using the definitions of $H$ and $G$ in Definition 2.39. But first, let us introduce the following lemmas:

LEMMA 2.50. For each $k \in\{0,1, \ldots, d-1\}, H_{k}(\mathbf{n})$ is monotonically increasing in each of its $d$ arguments, $\left\{n_{0}, n_{1}, \ldots, n_{d-1}\right\}$, for $\mathbf{n} \in \mathcal{E}$.

Proof of lemma. In $\mathcal{E}$ the conditions of monotonicity are satisfied. 'Thus, by definition $G_{k}(\mathrm{n}) \geq 0$, which implies that $I_{k}(\mathrm{n})$ is monotonically increasing. 
LFMMA 2.51. Suppose $\mathbf{n} \in$ and $\mathcal{C}\left(\mathbf{M}_{ \pm}\right)=0$. Then $H_{k}(\mathbf{n}) \in \mathcal{E}^{(k)}, \forall k \in$ $\{0,1 \ldots, d-1\}$.

Proof of lemma. Let $k \in\{0,1, \ldots, d-1\}$ be arbitrary. Then by Lemma 2.50 $I_{k}(n)$ is monotonically increasing in each of its $d$ arguments, $\left\{n_{0}, n_{1}, \ldots, n_{d-1}\right\}$, for $n \in \mathcal{E}$. Thus, the extreme values of $H_{k}(\mathbf{n})$ are at the extreme values of $\mathrm{n}$, i.e., at. $\mathbf{n}=\mathrm{M}_{ \pm}$. Recall from Definition 2.39 that $H_{k}(\mathbf{n})=n_{k}+\mathcal{C}_{k}(\mathbf{n})$. Then since $\mathcal{C}\left(\mathrm{M}_{ \pm}\right)=0$, it follows immediately that $H_{k}\left(\mathrm{M}_{ \pm}\right)=M_{ \pm}^{(k)}$ and $H_{k}(\mathbf{n}) \in \mathcal{E}^{(k)}$. Hence, $1 /$ maps from $\mathcal{E}$ to $\mathcal{E}$, i.c., $I$ applied to an argument in the domain of monotonicity produces a value in the domain of monotonicity.

Comollamy 2.52. $H: \mathcal{E} \rightarrow \mathcal{E}$

Proof of corollary. This follows immediately from that $H_{k}: \mathcal{E} \rightarrow \mathcal{E}^{(k)}$ for each $l: \in\{0,1, \ldots, d-1\}$,

Proof of Theorem 2.49. We now proced with the induction. Considering the basc step, since the case $n=0$ is an assumption of the theorem, we must show (for time step $n=1) n_{\vec{r}}^{1} \in \mathcal{E} \forall \vec{\imath} \in \mathcal{L}$. Well, for each lattice node $\vec{\imath}$, we apply Lemma 2.51 to obtain that $H_{k}\left(\mathbf{n}_{\mathbf{r}-\vec{e}_{k}}^{0}\right) \in \mathcal{E}^{(k)} \forall k \in\{0,1, \ldots, d-1\}$. $^{38}$ 'Therefore, $\mathrm{n}_{\mathfrak{r}}^{1}=\left[H_{k}\left(\mathbf{n}_{\vec{i}-\vec{c}_{k}}^{0}\right)\right]_{k=0}^{d-1} \in \mathcal{E}$.

For the induction step, we assume that $n_{i}^{n} \in \mathcal{E}$ to show $n_{i}^{n+1} \in \mathcal{E}$. Applying Lemma 2.51 for each $\vec{\imath} \in \mathfrak{L}$, we find $\left(n_{k}\right)_{i}^{n+1}=I_{k}\left(\mathrm{n}_{i-e_{k}}^{n}\right) \in \mathcal{E}^{(k)} \forall k \in\{0,1, \ldots, d-1\}$. Hence, $n_{i}^{n+1} \in \mathcal{E}$.

In summary, 'Theorem 2.49 establishes maximum and minimum principles for a lattice mothod under the following conditions:

1. (iiven that the intial condition is in the domain of monotonicity, and

\footnotetext{
"Wo have used that $n_{i}^{0} \in \mathcal{E}$ implies $n_{1}^{0 . \ldots f_{k}} \in \mathcal{E}$.
} 
2. At the extreme points of the domain of monotonicity (extreme is applied to each dimension separately), the lattice method is in a state of eqtilibrium, i.e., the collision operator is zero.

Example (LB1: Domain of Monotoni-ity).

Lemma 2.53 (Domain of Monotonicity Fon $\mathrm{LB}_{1}$ ), Let interval $\mathcal{E}^{(k)}=$ $\left[M_{-}, M_{+}\right]$for $k \in\{0,1,2,3\}$, where $M_{+}=(1+1 / \sqrt{5}) / 2$ and $M_{-}=(1-$ $1 / \sqrt{5}) / 2 .^{37}$ Then

$$
\mathcal{E}=\mathcal{E}^{(0)} \times \mathcal{E}^{(1)} \times \mathcal{E}^{(2)} \times \mathcal{E}^{(3)}
$$

is the domain of monotonicity for the lattice method, $L B_{1}$.

(Since the proof is rather long and involved, the reader may wish to accept the lemina and proceed to the discussion and development of the continuum maximum and minimum principles on $\mathrm{p} .110$.)

Proof. There are three parts to proving the lemma: (1) show that the conditions of monotonicity are satisfied in $\mathcal{E}$, (2) show that $\mathcal{E}$ cannot be extended to a larger connected region, and (3) show that $\mathcal{E}$ has volume. We will proceed in the order (3), (1), and (2).

(3) Clearly, $\mathcal{E}$ has volume since $M_{-}<M_{+}$.

(1) To show that the conditions of monotonicity are satisfied in $\mathcal{E}$, we begin by rewriting the Lattice Boltzrnann Equation for $L B_{1}$ in terms of $n^{n+1}$ to get

$$
\mathbf{n}_{\overrightarrow{\mathfrak{r}}}^{n+1}=\left[\left(n_{k}\right)_{\vec{i}-\vec{e}_{k}}^{n}+\mathcal{C}_{k}\left(\mathbf{n}_{\vec{T}-\vec{e}_{k}}^{n}\right)\right]_{k=0}^{k=3}
$$

where $\vec{\imath}=(i, j)$ and $\vec{e}_{k}$ is the unit velocity vector for direction $k$. Then $\|$ is

${ }^{37} M_{+} \approx 0.72361$ and $M_{-} \approx 0.276339$ 
defined by

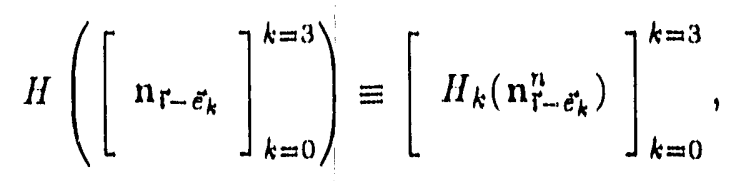

where

$$
H_{k}\left(\mathbf{n}_{\tau-\tilde{e}_{k}}^{n}\right) \equiv\left(n_{k}\right)_{\tau-e_{k}}^{n}+C_{k}\left(\mathbf{n}_{\tau_{-\tilde{e}_{k}}^{n}}^{n}\right)
$$

Then substituting for the $L B_{1}$ collision operator ( $E q .2 .10$ ), $H_{k}$ may be written explicitly,

$$
\begin{aligned}
\left(n_{k}\right)_{r}^{n+1} & =\left(n_{k}\right)_{i-e_{k}}^{n}+\mathcal{C}_{k}\left(\mathbf{n}_{r-e_{k}}^{n}\right) \\
& =H_{k}\left(\mathbf{n}_{r-e_{k}}^{n}\right) \\
& =\left(n_{k}\right)_{r-e_{k}}^{n} \\
& +\overline{\left(n_{k}\right)_{\tau-e_{k}}^{n}} \overline{\left(n_{k+1}\right)_{r-e_{k}}^{n}}\left(n_{k+2}\right)_{r-e_{k}}^{n}\left(n_{k+3}\right)_{r-e_{k}}^{n} \\
& +\overline{\left(n_{k}\right)_{r-e_{k}}^{n}}\left(n_{k+1}\right)_{r-e_{k}}^{n} \overline{\left(n_{k+2}\right)_{r-e_{k}}^{n}}\left(n_{k+3}\right)_{r-e_{k}}^{n} \\
& +\overline{\left(n_{k}\right)_{r-e_{k}}^{n}}\left(n_{k+1}\right)_{r-e_{k}}^{n}\left(n_{k+2}\right)_{r-e_{k}}^{n} \overline{\left(n_{k+3}\right)_{r-e_{k}}^{n}} \\
& -\left(n_{k}\right)_{r-e_{k}}^{n} \overline{\left(n_{k+1}\right)_{r-e_{k}}^{n}} \overline{\left(n_{k+2}\right)_{i-e_{k}}^{n}}\left(n_{k+3}\right)_{r-e_{k}}^{n} \\
& -\left(n_{k}\right)_{r-e_{k}}^{n} \overline{\left(n_{k+1}\right)_{r-e_{k}}^{n}}\left(n_{k+2}\right)_{i-e_{k}}^{n} \overline{\left(n_{k+3}\right)_{r-e_{k}}^{n}} \\
& -\left(n_{k}\right)_{r-e_{k}}^{n}\left(n_{k+1}\right)_{r-e_{k}}^{n} \overline{\left(n_{k+2}\right)_{r-e_{k}}^{n}} \overline{\left(n_{k+3}\right)_{r-e_{k}}^{n}} .
\end{aligned}
$$

Note that $\vec{\imath}-\vec{e}_{0}=(i+1, j), \vec{\imath}+\vec{e}_{1}=(i, j-1), \vec{\imath}-\vec{e}_{2}=(i-1, j)$, and $\vec{\imath}-\vec{e}_{3}=(i, j+1)$. To help clarlfy the notation, consider direction $k=0$.

Then

$$
\begin{aligned}
\left(n_{0}\right)_{i, j}^{n+1} & =\left(n_{0}\right)_{i-1, j}^{n}+\mathcal{C}_{0}\left(\mathbf{n}_{i-1, j}^{n}\right) \\
& =\left(n_{0}\right)_{i-1, j}^{n} \\
& +\overline{\left(n_{0}\right)_{i-1, j}^{n}} \overline{\left(n_{1}\right)_{i-1, j}^{n}}\left(n_{2}\right)_{i-1, j}^{n}\left(n_{3}\right)_{i-1, j}^{n} \\
& +\overline{\left(n_{0}\right)_{i-1, j}^{n}}\left(n_{1}\right)_{i-1, j}^{n} \overline{\left(n_{2}\right)_{i-1, j}^{n}}\left(n_{3}\right)_{i-1, j}^{n} \\
& +\overline{\left(n_{0}\right)_{i-1, j}^{n}}\left(n_{1}\right)_{i-1, j}^{n}\left(n_{2}\right)_{i-1, j}^{n} \overline{\left(n_{3}\right)_{i-1, j}^{n}}
\end{aligned}
$$




$$
\begin{aligned}
& -\left(n_{0}\right)_{i-1, j}^{n} \overline{\left(n_{1}\right)_{i-1, j}^{n}} \overline{\left(n_{2}\right)_{i-1, j}^{n}}-\left(n_{3}\right)_{i-1, j}^{n} \\
& -\left(n_{0}\right)_{i-1, j}^{n} \overline{\left(n_{1}\right)_{i-1, j}^{n}}\left(n_{2}\right)_{i-1, j}^{n} \overline{\left(n_{3}\right)_{i-1, j}^{n}} \\
& -\left(n_{0}\right)_{i-1, j}^{n}\left(n_{1}\right)_{i-1, j}^{n} \overline{\left(n_{2}\right)_{i-1, j}^{n}} \overline{\left(n_{3}\right)_{i-1, j}^{n}},
\end{aligned}
$$

There are similar equations for $\left(n_{1}\right)_{i, j}^{n+1},\left(n_{2}\right)_{i, j}^{n+1}$, and $\left(n_{3}\right)_{i, j}^{n+1}$. But because $L B_{1}$ is rotation invariant, we can concentrate on the general form of Eq. 2.50. Now, to prove that the conditions of monotonicity are satisfied in $\mathcal{E}$, we must show for

where

$$
G=\left[G_{k}\right]_{k=0}^{k=3}
$$

$$
G_{k}=\frac{\partial}{\partial \mathbf{n}_{\boldsymbol{i}-e_{k}}} H_{k}\left(\mathbf{n}_{\vec{i}-\tilde{e}_{k}}\right),
$$

that $G_{k} \geq 0$. In this proof, for the sake of brevity, it is understood that unless explicitly stated expressions involving $n_{\hat{k}}$ for $\hat{k} \in\{k, k+1, k+2, k+3\}$ are all evaluated at $\left(\vec{\imath}-\vec{e}_{k} ; n\right)$. Then for example, $n_{k+1}$ denotes $\left(n_{k+1}\right)_{r_{-} \vec{e}_{k}}^{n}$; similarly, $\mathbf{n}$ denotes $\mathbf{n}_{i-e_{k}}^{n}$. Then with $k$ and $l$ evaluated modulo $d=4$, we have

$$
G_{k, l}=\frac{\partial}{\partial\left(n_{l}\right)_{i-e_{k}}} H_{k}\left(\mathbf{n}_{r-e_{k}}\right) .
$$

Then we find

$$
\begin{aligned}
G_{k, k} & =\frac{\partial}{\partial\left(n_{k}\right)_{\boldsymbol{i}-\vec{e}_{k}}} H_{k}\left(\mathrm{n}_{\vec{i}-\vec{e}_{k}}\right) \\
& =1+n_{k+2} n_{k+3}+n_{k+1} n_{k+3}-n_{k+3}+n_{k+1} n_{k+2}-n_{k+2}-n_{k+1} \\
G_{k, k+1} & =\frac{\partial}{\partial\left(n_{k+1}\right)_{i-\vec{e}_{k}}} H_{k}\left(\mathrm{n}_{\vec{i}-\vec{e}_{k}}\right) \\
& =-3 n_{k+2} n_{k+3}+n_{k} n_{k+3}+n_{k+3}+n_{k} n_{k+2}+n_{k+2}-n_{k} \\
G_{k, k+2} & =\frac{\partial}{\partial\left(n_{k+2}\right)_{i-\vec{e}_{k}}} I_{k}\left(\mathrm{n}_{\vec{i}-\vec{e}_{k}}\right) \\
& =-3 n_{k+1} n_{k+3}+n_{k} n_{k+3}+n_{k+3}+n_{k} n_{k+1}+n_{k+1}-n_{k}
\end{aligned}
$$




$$
\begin{aligned}
G_{k, k+3} & =\frac{\partial}{\partial\left(n_{k+3}\right)_{i-\vec{e}_{k}}} H_{k}\left(n_{i-\vec{e}_{k}}\right) \\
& =-3 n_{k+1} n_{k+2}+n_{k} n_{k+2}+n_{k+2}+n_{k} n_{k+1}+n_{k+1}-n_{k} .
\end{aligned}
$$

(Note that these partial derivatives were calculated when determining the linearized collision operator, ${ }^{38}$ ) We show that $G_{k} \geq 0$ by showing that $G_{k, 1} \geq$ 0 .

Note that each $G_{k, l}$ is a function of three arguments. Let $E \equiv\left[M_{-}, M_{+}\right]$. Therl the domain of a particular $G_{k, l}$ is $E^{3}$, a box. Toward the end of reducing the number of cases to check, let

$$
\begin{aligned}
& f(\zeta, \rho, \phi)=1+\rho \phi+\zeta \phi+\zeta \rho-\zeta-\rho-\phi, \\
& g(\zeta, \rho, \phi)=-3 \rho \phi+\zeta \phi+\zeta \rho+\rho+\phi-\zeta .
\end{aligned}
$$

Referring to Eqs. 2.51-2.55, we see that

$$
\begin{aligned}
& G_{k, k}=f\left(n_{k+1}, n_{k+2}, n_{k+3}\right), \\
& G_{k, k+1}=g\left(n_{k}, n_{k+2}, n_{k+3}\right), \\
& G_{k, k+2}=g\left(n_{k}, n_{k+1}, n_{k+3}\right), \\
& G_{k, k+3}=g\left(n_{k}, n_{k+1}, n_{k+2}\right) .
\end{aligned}
$$

The domain of $f$ and $g$ is $E^{3}$. To prove $\left(i_{k, l} \geq 0\right.$, it is enough to show that $f(\zeta, \rho, \phi), g(\zeta, \rho, \phi) \geq 0$ for $(\zeta, \rho, \phi) \in E^{3}$. We show that the extreme values of $\int$ and $g$ are nonnegative.

Since $f, g \in C^{1}\left(\mathcal{R}^{3}, \mathcal{R}\right)$, their respective absolute extrema are on the bound. ary of $E^{3}$, i.e., $\partial E^{3}$ (on the edges and faces of the box), or where the gradients of $f$ and $y$ are zero in the interior of $E^{3}$, i.e., $E^{3} \backslash \partial E^{3}$.

\footnotetext{
${ }^{38}$ ef. Eess. 2.52-2.55 hefore the evaluation of the partial derivatives at the equilibrium state, i.e., before substituting $\mathrm{n}=\mathbf{n}^{(0)}=u(1)$.
} 
Local Extrema of $f$ and $g$. We show that for $(\zeta, \rho, \phi) \in E^{3} \backslash \partial E^{3}$, the local extreme value of $f$ and $g$ is $1 / 4$. It is found at $(\zeta, \rho, \phi)=(1 / 2,1 / 2,1 / 2)$. Consider $(\zeta, \dot{\zeta}, \phi) \in E^{3} \backslash \partial E^{3}$. Then the local extrema of $f$ and $g$ are found where $\nabla_{\zeta, \rho, \phi} f(\zeta, \rho, \phi)=(0,0,0)$ and $\nabla_{\zeta, \rho, \phi} g(\zeta, \rho, \phi)=(0,0,0)$, respec. tively.

We find

$$
\begin{aligned}
& \nabla_{\zeta, \rho, \phi} f(\zeta, \rho, \phi)=(\rho+\phi-1, \zeta+\phi-1, \zeta+\rho-1) \\
& \nabla_{\zeta, \rho, \phi} g(\zeta, \rho, \phi)=(\rho+\phi-1,-3 \phi+\zeta+1,-3 \rho+\zeta+1) .
\end{aligned}
$$

Then setting the gradients to zero, we find for $f$ that $\zeta=\rho=\phi=1 / 2$ and for $g$ that $\zeta=\rho=\phi=1 / 2$. Hence, the local extreme values are

$\begin{aligned} & f(1 / 2,1 / 2,1 / 2)=1 / 4, \\ & g(1 / 2,1 / 2,1 / 2)=1 / 4 . \\ & \text { Thus, } G_{k, l} \text { has a local extremurn at } \mathrm{n}_{e q}=\left[\begin{array}{c}1 / 2 \\ 1 / 2 \\ 1 / 2 \\ 1 / 2\end{array}\right] \text {. The local extreme values }\end{aligned}$ are given by

$$
G_{k, l}\left(\mathbf{n}_{e q}\right)=1 / 4, \quad \forall k, l \in\{0,1,2,3\}
$$

Boundary Extrema of $f$ and $g$. We will show that the boundary extrema of $f$ and $g$ are between 0 and 2/5. For each function, $f$ and $g$, there are six cases:

$$
\begin{array}{ll}
\text { 1a/b: } & (b, \rho, \phi), \quad b \in\left\{M_{-}, M_{+}\right\}, \\
\text {2a/b: } \quad(\zeta, \rho, b), \quad b \in\left\{M_{-}, M_{+}\right\}, \\
\text {3a/b: } \quad(\zeta, b, \phi), \quad b \in\left\{M_{-}, M_{+}\right\} .
\end{array}
$$


Noting that $f(\zeta, \rho, \phi)=f(\zeta, \phi, \rho)$ and $g(\zeta, \rho, \phi)=g(\zeta, \phi, \rho)$, the number of cases is reduced to four for each function. Checking cases $1 a / b$ anc $2 a / b$ will suffice.

Case $1 \mathrm{a} / \mathrm{b}:$ Consider $(b, \rho, \phi)$ with $b \in\left\{M_{-}, M_{+}\right\}$and $(\rho, \phi) \in E^{2}$. Then

$$
\begin{aligned}
& f(b, \rho, \phi)=\rho \phi+b \rho+b \phi-\rho-\phi-b+1 \\
& g(b, \rho, \phi)=-3 \rho \phi+b \rho+b \phi+\rho+\phi-b .
\end{aligned}
$$

We now consider $f$ and $g$ as functions of $\rho$ and $\phi$. The local extreme values of these $f$ and $g$ are where their gradients (in $\rho$ and $\phi$ ) are zero. For $f$ we find

$$
\nabla_{\rho, \phi} f(b, \rho, \phi)=(\phi+b-1, \rho+b-1)=(0,0)
$$

implies that $\rho=\phi$. Then

$$
f(b, \rho, \rho)=\rho^{2}+2(b-1) \rho-b+1
$$

and (treating $f(b, \rho, \rho)$ as a function of $\rho$ )

$$
\frac{\partial}{\partial \rho} f(b, \rho, \rho)=2 \rho+2(b-1)=0
$$

implies that $\rho=1-b$. The local extrema of $f(b, \rho, \phi)$ are thus found at $(b, a, c)$ for $a, b, c \in\left\{M_{-}, M_{+}\right\}$. The results are listed in Table 2.5. For $g$ we find

$$
\nabla_{\rho, \phi} g(b, \rho, \phi)=(-3 \phi+b+1,-3 \rho+b+1)=(0,0)
$$

implies that $\rho=\phi$. Then

$$
g(b, \rho, \rho)=-3 \rho^{2}+2(b+1) \rho-b
$$

and (treating $g(b, \rho, \rho)$ as a function of $\rho$ )

$$
\frac{\partial}{\partial \rho} g(b, \rho, \rho)=2(-3 \rho+b+1)=0
$$


implies that $\rho=(b+1) / 3$. The local extrema of $g(b, \rho, \phi)$ are thus found at $(b,(b+1) / 3,(b+1) / 3)$ for $b \in\left\{M_{-}, M_{+}\right\}$. We find

$$
\begin{aligned}
& g\left(M_{--},\left(M_{-}+1\right) / 3,\left(M_{-}+1\right) / 3\right)=4 / 15 \\
& g\left(M_{+},\left(M_{+}+1\right) / 3,\left(M_{+}+1\right) / 3\right)=4 / 15 .
\end{aligned}
$$

The boundary extrema are found at $(b, a, c)$ for $a, b, c \in\left\{M_{-}, M_{+}\right\}$and are listed in Table 2.5.

Case 2a/b: Consider $(\zeta, \rho, b)$ with $b \in\left\{M_{-}, M_{+}\right\}$and $(\zeta, \rho) \in E^{2}$. Then

$$
\begin{aligned}
& f(\zeta, \rho, b)=\zeta \rho+b \zeta+b \rho-\zeta-\rho-b+1, \\
& g(\zeta, \rho, b)=-3 b \rho+b \zeta+\zeta \rho-\zeta+\rho+b .
\end{aligned}
$$

We now consider $f$ and $g$ as functions of $\zeta$ and $\rho$. The local extreme values of $f$ and $g$ are where their gradients (in $\zeta$ and $\rho$ ) are zero. For $f$ we find

$$
\nabla_{\zeta, \rho} f(\zeta, \rho, b)=(\rho+b-1, \zeta+b-1)=(0,0)
$$

implies that $\zeta=\rho$. Then

$$
f(\zeta, \zeta, b)=\zeta^{2}+2(b-1) \zeta-b+1
$$

and (treating $f(\zeta, \zeta, b)$ as a function of $\zeta$ )

$$
\frac{\partial}{\partial \zeta} f(\zeta, \zeta, b)=2 \zeta+2(b-1)=0
$$

implies that $\zeta=1-b$. The local extrema of $f(\zeta, \rho, b)$ are thus found at $(a, c, b)$ for $a, b, c \in\left\{M_{-}, M_{+}\right\}$. The results are listed in Table 2.5. For $g$ we find

$$
\nabla_{\zeta, \rho} g(\zeta, \rho, b)=(\rho+b-1, \zeta-3 b+1)=(0,0)
$$


Table 2.5: Boundary Extrema of $f(\zeta, \rho, \phi)$ and $g(\zeta, \rho, \phi)$ in $\mathrm{LB}_{1}$.

\begin{tabular}{|c|c|c|c|c|}
\hline$\zeta$ & $\rho$ & $\phi$ & $\zeta, \rho, \phi$ & $g(\zeta, \rho, \phi)$ \\
\hline$\overline{M_{-}}$ & $M$ & $\overline{M_{-}}$ & $2 / 5$ & $1 / 5$ \\
\hline$M_{-}$ & $M$ & $M_{+}$ & $1 / 5$ & $2 / 5$ \\
\hline$M_{-}$ & $M_{\uparrow}$ & $M_{-}$ & $1 / 5$ & $2 / 5$ \\
\hline$M_{-}$ & $M_{\uparrow}$ & $M_{+}$ & $1 / 5$ & 0 \\
\hline$M_{+}$ & $M$ & $M_{-}$ & $1 / 5$ & 0 \\
\hline$M_{+}$ & $M$ & $M_{+}$ & $1 / 5$ & $2 / 5$ \\
\hline$M_{+}$ & $M_{-}$ & $M_{-}$ & $1 / 5$ & $2 / 5$ \\
\hline$M_{+}$ & $M_{-}$ & $M_{+}$ & $2 / 5$ & $1 / 5$ \\
\hline
\end{tabular}

implies that $\rho=\zeta-4 b+2$. Then

$$
g(\zeta, \zeta-4 b+2, b)=\zeta^{2}+2(1-3 b) \zeta-9 b+2+12 b^{2}
$$

and (treating $g(\zeta, \zeta-4 b+2, b)$ as a function of $\zeta$ )

$$
\frac{\partial}{\partial \zeta} g(\zeta, \zeta-4 b+2, b)=2(\zeta-3 b+1)=0
$$

implies that $\zeta=3 b-1$. But $3 b-1 \notin E$ for $b \in\left\{M_{-}, M_{+}\right\}$. Hence, this case does not produce a local extreme value inside the domain of $\zeta$, i.e., $E \backslash \partial E$. And noting that $a-4 b+2 \notin E$ for $a, b \in\left\{M_{-}, M_{+}\right\}$implies that there are no extreme values on the boundary of the domain of $\zeta$ either. So, $g(\zeta, \rho, b)$ has no local extrema. However, boundary extrema are found at $(a, c, b)$ for $a, b, c \in\left\{M_{-}, M_{+}\right\}$. They are listed in Table 2.5 .

Absolute Extrema of $G_{k, l}$. The absolute extrema of $f$ and $g$ are found as the respective minimum and maximum over the local and boundary extreme values. Considering the preceding analysis, we find for $(\zeta, \rho, \phi) \in E^{3}$,

$$
\begin{aligned}
& \min f(\zeta, \rho, \phi)=1 / 5, \quad \max f(\zeta, \rho, \phi)=2 / 5, \\
& \min g(\zeta, \rho, \phi)=0, \quad \max g(\zeta, \rho, \phi)=2 / 5 .
\end{aligned}
$$


Thus,

$$
\min G_{k, l}=0 \quad \text { and } \quad \max G_{k, l}=\frac{2}{5} .
$$

Hence, Eq. 2.41 holds, and everywhere in $\mathcal{E}$ are the conditions of monotonicity satisfied.

(2) To show $\mathcal{E}$ cannot be extended. Let $\epsilon>0$ be given such that $0 \leq M_{--}-\epsilon<$ $M_{+}+\epsilon \leq 1$. Then let $\mathbf{R}_{-} \equiv\left[M_{-}-\epsilon, M_{+}\right]$and $\mathbf{R}_{+} \equiv\left[M_{-}, M_{+}+\epsilon\right]$, and $\mathbf{M}=\left[M_{-}, M_{+}\right]$. Further, let $\mathbf{E}_{ \pm} \equiv \mathbf{M} \cup \mathbf{E}_{ \pm}$. Then let

$$
\begin{aligned}
& \hat{\mathcal{E}}_{0, \pm} \equiv \mathrm{M} \times \mathrm{M} \times \mathrm{M} \times \mathrm{E}_{ \pm}, \\
& \hat{\mathcal{E}}_{1, \pm} \equiv \mathrm{M} \times \mathrm{M} \times \mathbf{E}_{ \pm} \times \mathbf{M}, \\
& \hat{\mathcal{E}}_{2, \pm} \equiv \mathrm{M} \times \mathrm{E}_{ \pm} \times \mathbf{M} \times \mathbf{M}, \\
& \hat{\mathcal{E}}_{3, \pm} \equiv \mathbf{E}_{ \pm} \times \mathbf{M} \times \mathbf{M} \times \mathbf{M} .
\end{aligned}
$$

It is enough to show that for each $i \in\{0,1,2,3\}$ and $s \in\{+,-\}, G_{k, l}\left(\mathrm{n}^{*}\right)<$ 0 for some $k, l \in\{0,1, \ldots, d-1\}$ and some $\mathbf{n}^{*} \in \hat{\mathcal{E}}_{i, s}$. Well, note that from Eq. 2.56 and Table 2.5, $G_{0,1}=0$ for (1) $n_{0}=M_{-}$, and $n_{2}=n_{3}=M_{+}$, and (2) $n_{0}=M_{+}$, and $n_{2}=n_{3}=M_{-}$. There are eight cases to examine. While we will only present the proof for two cases, the other cases proceed similarly, and the tables we will be using have enough information to verify the other six cases. For each case we use a proof by contradiction.

Case $\hat{\mathcal{E}}_{0,:}=\mathrm{M} \times \mathrm{M} \times \mathrm{M} \times \mathrm{E}_{+}:$Suppose $G_{k, l} \geq 0, \quad \forall k, l \in\{0,1,2,3\}$ and $\forall \mathbf{n} \in \hat{\mathcal{E}}_{0,+}$. Let

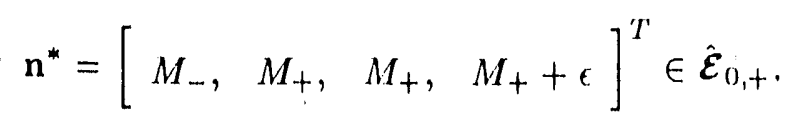

Note that $G_{0,1}\left(\mathbf{n}^{*}\right)=g\left(M_{-}, M_{+}, M_{+}+\epsilon\right)$. From Table 2.6 we see that $\frac{\partial}{\partial \epsilon} g\left(M_{-}, M_{+}, M_{+}+\epsilon\right)<0$. This coupled with $g\left(M_{-}, M_{+}, M_{+}\right)=0$ 
(see Table 2.5) implies that $G_{0,1}\left(\mathrm{n}^{*}\right)<0$, which is a contradiction.

Therefore, the conditions of monotonicity are not everywhere satisfied in $\hat{\boldsymbol{\varepsilon}}_{0,+}$

Case $\hat{\mathcal{E}}_{0,-}=\mathrm{M} \times \mathrm{M} \times \mathrm{M} \times \mathrm{E}_{-}:$Suppose $G_{k, l} \geq 0, \forall k, l \in\{0,1,2,3\}$ and $n \in \hat{\mathcal{E}}_{0, \ldots}$. Let

$$
\mathbf{n}^{*}=\left[\begin{array}{llll}
M_{+}, & M_{-}, & M_{-}, & M_{-}-\epsilon
\end{array}\right]^{T} \in \hat{\varepsilon}_{0,-}
$$

Note that $G_{0,1}\left(\mathrm{n}^{*}\right)=g\left(M_{+}, M_{-}, M_{-}-\epsilon\right)$. From Table 2.6 we see that $\frac{\partial}{\partial \epsilon} g\left(M_{+}, M_{-}, M_{-}-\epsilon\right)<0$. This coupled with $g\left(M_{+}, M_{-}, M_{-}\right)=0$ (see Table 2.5) implies that $G_{0,1}\left(\mathbf{n}^{*}\right)<0$, which is a contradiction. Therefore, the conditions of monotonicity are not everywhere satisfied in $\hat{\boldsymbol{\varepsilon}}_{0, \ldots}$.

As noted, the remaining cases proceed similarly, the final result being that none of the eight ways of extending $\mathcal{E}$ produces a region everywhere satisfying the conditions of monotonicity.

We have shown that the conditions of monotonicity are satisfied in $\mathcal{E}$, that no extension of $\mathcal{E}$ everywhere satisfies the conditions of monotonicity, and that $\mathcal{E}$ has volume. Hence, $\mathcal{E}=[(1-1 / \sqrt{5}) / 2,(1+1 / \sqrt{5}) / 2]^{4}$ is the domain of monotonicity for $L B_{1}$.

Lemma 2.54 (Discrete Maximum/Minimum Principle for LB 1 ). Considering the lattice method, $L B_{1}$, let its domain of monotonicity, $\mathcal{E}$, be as in the hypothesis of Lemma 2.53. Then LB, has the discrete maximum and minimum principles described in Theorem 2.49.

Proof. Note that $\mathbf{M}_{+}=M_{+} \mathbf{q}_{0}$ and $\mathbf{M}_{-}=M_{-} \mathbf{q}_{0}$, and that $\mathcal{C}_{k}\left(\mathbf{M}_{+}\right)=$ $\mathcal{C}_{k}\left(\mathbf{M}_{-}\right)=0 \forall k \in\{0,1,2,3\}$. Therefore, Theorem 2.49 applies, i.e., if $\mathbf{n}_{\mathbf{i}}^{0} \in \mathcal{E} \forall$ $\vec{\imath} \in \mathfrak{L}$, then $\mathbf{n}_{\vec{i}}^{n} \in \mathcal{E} \forall \vec{\imath} \in \mathfrak{L}$ for all time steps $n$. 
Table 2.6: Direction of Increase in $f$ and $g$ for Arguments Just Outside $\mathcal{E}$ in $\mathrm{LB}_{1}$. The table lists $\frac{\partial}{\partial \epsilon} f$ and $\frac{\partial}{\partial \epsilon} g$ for arguments just outside the boundary of $\mathcal{E}$. The arguments are parameterized by $\epsilon>0$.

\begin{tabular}{|c|c|c|c|c|}
\hline \multicolumn{3}{|c|}{$\vec{r}(\epsilon)=(\zeta(\epsilon), \rho(\epsilon), \phi(\epsilon))$} & \multirow{2}{*}{$\frac{\partial}{\partial \epsilon} f(\vec{r}(\epsilon))$} & \multirow{2}{*}{$\frac{\partial}{\partial \epsilon} g(\vec{r}(\epsilon))$} \\
\hline$\zeta(\epsilon)$ & $\rho(\epsilon)$ & $\phi(\varepsilon)$ & & \\
\hline$M_{-}$ & $M_{-}$ & $M_{-}-\epsilon$ & $+1 / \sqrt{5}$ & $-1 / \sqrt{5}$ \\
\hline$M_{-}$ & $M_{-}$ & $M_{+}+\epsilon$ & $-1 / \sqrt{5}$ & $+1 / \sqrt{5}$ \\
\hline$M_{-}$ & $M_{+}$ & $M_{-}-\epsilon$ & 0 & $+2 / \sqrt{5}$ \\
\hline$M_{-}$ & $M_{+}$ & $M_{+}+\epsilon$ & 0 & $-2 / \sqrt{5}$ \\
\hline$M_{+}$ & $M_{-}$ & $M_{-}-\epsilon$ & 0 & $-2 / \sqrt{5}$ \\
\hline$M_{+}$ & $M_{-}$ & $M_{+}+\epsilon$ & 0 & $+2 / \sqrt{5}$ \\
\hline$M_{+}$ & $M_{+}$ & $M_{-}-\epsilon$ & $-1 / \sqrt{5}$ & $+1 / \sqrt{5}$ \\
\hline$M_{+}$ & $M_{+}$ & $M_{+}+\epsilon$ & $+1 / \sqrt{5}$ & $-1 / \sqrt{5}$ \\
\hline$M_{-}$ & $M_{-}-\epsilon$ & $M_{-}$ & $+1 / \sqrt{5}$ & $-1 / \sqrt{5}$ \\
\hline$M_{-}$ & $M_{-}-\epsilon$ & $M_{+}$ & 0 & $+2 / \sqrt{5}$ \\
\hline$M_{-}$ & $M_{+}+\epsilon$ & $M_{-}$ & $-1 / \sqrt{5}$ & $+1 / \sqrt{5}$ \\
\hline$M_{-}$ & $M_{+}+\epsilon$ & $M_{+}$ & 0 & $-2 / \sqrt{5}$ \\
\hline$M_{+}$ & $M_{-}-\epsilon$ & $M_{-}$ & 0 & $-2 / \sqrt{5}$ \\
\hline$M_{+}$ & $M_{-}-\epsilon$ & $M_{+}$ & $-1 / \sqrt{5}$ & $+1 / \sqrt{5}$ \\
\hline$M_{+}$ & $M_{+}+\epsilon$ & $M_{-}$ & 0 & $+2 / \sqrt{5}$ \\
\hline$M_{+}$ & $M_{+}+\epsilon$ & $M_{+}$ & $+1 / \sqrt{5}$ & $-1 / \sqrt{5}$ \\
\hline$M_{-}-\epsilon$ & $M_{-}$ & $M_{-}$ & $+1 / \sqrt{5}$ & $+1 / \sqrt{5}$ \\
\hline$M_{-}-\epsilon$ & $M_{-}$ & $M_{+}$ & 0 & 0 \\
\hline$M_{-}-\epsilon$ & $M_{+}$ & $M_{-}$ & 0 & 0 \\
\hline$M_{-}-\epsilon$ & $M_{+}$ & $M_{+}$ & $-1 / \sqrt{5}$ & $-1 / \sqrt{5}$ \\
\hline$M_{+}+\epsilon$ & $M_{-}$ & $M_{-}$ & $-1 / \sqrt{5}$ & $-1 / \sqrt{5}$ \\
\hline$M_{+}+\epsilon$ & $M_{-}$ & $M_{+}$ & 0 & 0 \\
\hline$M_{+}+\epsilon$ & $M_{+}$ & $M_{-}$ & 0 & 0 \\
\hline$M_{+}+\epsilon$ & $M_{+}$ & $M_{+}$ & $+1 / \sqrt{5}$ & $+1 / \sqrt{5}$ \\
\hline
\end{tabular}


We have established maximum and minimum principles for the Lattice Boltzmann Equation for L.B. The initial requirement is that

$$
\frac{1-1 / \sqrt{5}}{2} \leq\left(n_{k}\right)_{i}^{0} \leq \frac{1+1 / \sqrt{5}}{2}
$$

for all lattice nodes $\vec{\imath}$ and directions $k \in\{0,1,2,3\}$, i.e., $\|\mathbf{n}-1 / 2\|_{\ell_{\infty}} \leq 1 / \sqrt{5}$.

\section{Continuum Maximum/Minimum Principle}

Recall that $\mathrm{h}$ is a truncated expansion about an equilibrium so that $\mathrm{h}=\sum_{j=0}^{J} \mathrm{~h}^{(j)}$ for some finite $J$. Note that the $\mathbf{h}^{(j)}$ are in terms of the $\sigma^{(l)}(l \leq j)$ parameters introduced in determining the corresponding $\mathbf{n}^{(j)}$ in the discrete Chapman-Enskog expansion on n. The $\sigma^{(0)}$ parameters are solutions of the $\mathcal{O}\left[\delta^{2}\right]$ consistency conditions, i.e., the hydrodynamical equations. The $\sigma^{(l)}$ parameters (for $l \in\{1,2, \ldots, J\}$ ) satisfy the $\mathcal{O}\left[\delta^{l+2}\right]$ consistency conditions. Thus, maximum and minimum principles for $\mathrm{h}$ can be obtained from (1) maximum and minimum principles for the $\sigma^{(0)}$ parameters, and (2) regularity of all $\sigma^{(l)}$ parameters appearing in the truncated equilibrium expansion, i.e., h, and (3) conditions on $\delta$.

Example ( $L B_{1}$ : Continuum Maximum and Minimum Principles).

We now present the continuum maximum/minimum principle for $L B_{1}$. The main results are in Lemma 2.56 and Corollary 2.57.

Lemma 2.55 (Hydrodynamical Maximum/Minimum Principle for $\left.\mathrm{LB}_{1}\right)$. Let $u(x, y ; t)$ be a solution of

$$
\frac{\partial}{\partial t} u=\nu \nabla \cdot D(u) \nabla u \text { on } \Omega=([0, L] \times[0, L]) \times(0, T],
$$

where $D(u)=\frac{1}{4 u(1-u)}-\frac{1}{2}$ with initial condition, $u(x, y ; 0)=u_{I}(x, y)$ satisfying $0<\tilde{R}_{-} \leq u(x, y ; 0) \leq \tilde{R}_{+}<1$, for some $R_{-}, R_{+} \in(0,1)$ such that 
$R_{-}<R_{+}$, and periodic boundary conditions. Then the maximum and minimum of $u(x, y ; t)$ occur either at the initial time, i.e., $\tilde{R}_{-} \leq u(x, y ; t) \leq \tilde{R}_{+} \forall$ $(x, y ; t) \in([0, L] \times[0, L]) \times[0, T]$.

Proof. Define a new coefficient, $\dot{D}(u)$, that is precisely $D(u)$ on the closed interval $\left[\tilde{R}_{-.}, \tilde{R}_{+}\right]$and smoothly extends $D(u)$ beyond the closed interval to all of $\mathcal{R}$ so that $\hat{D}(u)$ has everywhere a bounded first gradient and $\hat{D}(u)$ is a positive function. Now, let $\hat{\imath}$ be a solution of

$$
\frac{\partial}{\partial t} \hat{\imath}=\nu \nabla \cdot(\hat{D}(\hat{\imath}) \nabla \hat{\imath})
$$

with periodic boundary conditions and initial condition satisfying $\hat{R}_{-} \leq \hat{u}(x, y ; 0) \leq$ $\hat{R}_{+}$for all $(x, y) \in[0, L] \times[0, L]$ and $0<\hat{R}_{-}<\hat{R}_{+}<1$. Then since Eq. 2.58 is easily verified to be parabolic for all $\hat{u}$ and any constant satisfies that equation, Theorem 12 of $\left[63\right.$, p. 187-8] applies to Eq. 2.58. The result is that $\hat{R}_{-} \leq \hat{u}(x, y ; t) \leq$ $\hat{R}_{+}$for $(x, y ; t) \in([0, L] \times[0, L]) \times[0, T]$, establishing a maximum/minimum prin. ciple for Eq. 2.58 .

Now, suppose $\hat{u}(x, y ; 0) \equiv u_{l}(x, y)$, and $\hat{R}_{-} \equiv \tilde{R}_{-}$and $\hat{R}_{+} \equiv \tilde{R}_{+}$. Then the solutions of Eq. 2.57 and Eq. 2.58 are identical, i.e., $\hat{u}(x, y ; t)=u(x, y ; t)$. Therefore,

$$
\tilde{R}_{-} \leq u(x, y ; t) \leq \tilde{R}_{+} \forall(x, y ; t) \in([0, L] \times[0, L]) \times[0, T]
$$

where $0<\tilde{R}_{-} \leq \tilde{R}_{+}<1$.

We have established a maximum/minimum principle for the hydrodynamical equation of $L B_{1}$ (Eq. 2.29).

We arrive at the following maximum/minimum principle for $h$.

Lemma 2.56 (Continuem Maximum/Minimum Principle for la $B_{1}$ ). Let $u(x, y ; t)$ be a solution of Eq. 2.29, and let $\mathrm{h}=\sum_{j=0}^{3} \delta^{j} \mathrm{~h}^{(j)}$ be as in the 
hypothesis of leemma $2.48^{30}$ Sippose for some constants $R_{\ldots}, R_{+}, B^{(1)}, B^{(2)}$, and $B^{(3)}$,

1. $0<R_{-}<u(x, y ; 0)<R_{+}<1 \forall(x, y) \in[0, L] \times[0, L]$, and

2. $\left\|\mathrm{h}^{(i)}\right\|_{C_{\infty}} \leq B^{(j)}<\infty \forall(x, y ; t) \in([0, L] \times[0, L]) \times[0, T], \forall j \in\{1,2,3\}$.

Then $\exists \delta_{0}>0$ such that for $\delta \in\left(0, \delta_{0}\right)$

$$
\left\|\mathrm{h}^{n}-\frac{R_{+}+R_{-}}{2}\right\|_{\ell_{\infty}} \leq \frac{R_{+}-R_{-}}{2} \leq \frac{1}{\sqrt{5}}
$$

i.e., $\mathrm{h}^{n} \in\left[R_{-}, R_{+}\right]^{d}, \forall$ time steps $n \geq 0$.

Proof. Well, by Lemma 2.55 with $\tilde{R}_{-} \equiv \inf u(x, y ; 0)$ and $\tilde{R}_{+} \equiv \sup u(x, y ; 0)$ so that $R_{-}<\tilde{R}_{-} \leq \tilde{R}_{+}<R_{+}$, we have

$$
\left\|\mathrm{h}^{(0)}-\frac{R_{+}+R_{-}}{2}\right\|_{\ell_{\infty}}<\frac{R_{+}-R_{-}}{2} \forall(x, y ; t) \in([0, L] \times[0, L]) \times[0, T] .
$$

Consequently,

$$
R_{-}<\left(h_{k}^{(0)}\right)_{r}^{n}<R_{+}
$$

for all lattice nodes $\vec{\imath} \in \mathcal{L}$, time steps $n$, and directions $k \in\{0,1,2,3\}$.

Now, choose $\delta_{0}>0 \ni$ for $\delta \in\left(0, \delta_{0}\right), R_{-} \leq\left(h_{k}^{(0)}\right)_{i}^{n}+\delta B^{(1)}+\delta^{2} B^{(2)}+\delta^{3} B^{(3)} \leq$

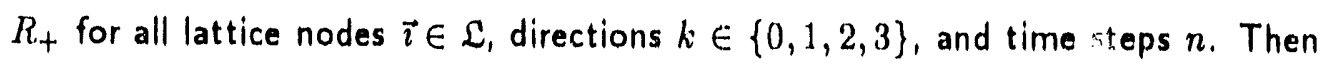
for $\delta \in\left(0, \delta_{0}\right)$ we have

$$
\begin{aligned}
\left\|\mathrm{h}^{n}-\frac{R_{+}+R_{ \pm}}{2}\right\|_{\ell_{\infty}} & =\left\|\left(\mathbf{h}^{(0)}\right)^{n}+\delta\left(\mathbf{h}^{(1)}\right)^{n}-\frac{R_{+}+R_{-}}{2}\right\|_{\ell_{\infty}} \\
& \leq\left\|\left(\mathbf{h}^{(0)}\right)^{n}-\frac{R_{+}+R_{-}}{2}\right\|_{\ell_{\infty}}+\sum_{j=1}^{3} \delta^{j}\left\|\left(\mathbf{h}^{(j)}\right)^{n}\right\|_{\ell_{\infty}} \\
& \leq\left\|\left(\mathbf{h}^{(0)}\right)^{n}-\frac{R_{+}+R_{-}}{2}\right\|_{\ell_{\infty}}+\sum_{j=1}^{3}\left(\delta_{0}\right)^{j} B^{(j)} \\
& \leq \frac{R_{+}-R_{-}}{2} .
\end{aligned}
$$

\footnotetext{
${ }^{39 t}$ The coefficients, $h^{(j)}, j \in\{0,1,2,3\}$, are defined in Eqs. 2.43-2.46, respectively.
} 
Hence, $\mathrm{h}^{n} \in\left[R_{-}, R_{+}\right]^{d}$,

Corohiary 2.57. In $L B_{1}, \exists \delta_{0}>0$, whech that $\forall \delta \in\left(0, \delta_{0}\right)$, if $h_{1}^{0} \in \mathcal{E} \backslash 0 \mathcal{E}$, $\forall \vec{\imath} \in \mathcal{L}$, then $h_{\mathfrak{r}}^{n} \in \mathfrak{E}, \forall \vec{\imath} \in \mathcal{L}, \forall n$, where $\mathcal{E}$ is the domuin of monotonirity for $L B_{1}$.

Proof, Let $R_{+} \equiv M_{+}<1$ and $R_{-} \equiv M_{-}>0$, where $M_{+}$and $M_{-}$are defined in Lemma 2.53 . Then apply Lemma 2.56 to yield the result.

We have established a continuum maximum and minimum principle for the truncated equilibrium expansion, $h$. The sufficient conditions are that $(1) \sigma^{(0)}$ has

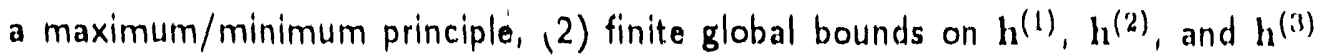
exist ${ }^{40}$, and (3) expansion parameter $\delta>0$ is sufficiently small.

\subsubsection{Stability}

THEOREM 2.58 (STABIITY IN THE $L_{1}$-NORM), Let a lattice method be given with domain of monotonicity, $\mathcal{E}$, Let $\mathrm{n}_{\mathrm{r}}^{n}$ and $\mathrm{h}_{\mathrm{p}}^{n}$. Suppose that if $\mathrm{h}_{\uparrow}^{0} \in \mathcal{E}$, then $\mathrm{h}_{\mathrm{p}}^{\mathrm{n}} \in \mathcal{E}$ $\forall n$ such that $0 \leq n \Delta t \leq T^{\prime}$ and $\Delta t<\tau$ for some $\tau>0$. Then if the method is mass-conserving it is stable $e^{41}$ in the $L_{1}-n o r m$.

Lemma 2.59. Let $\mathcal{C}(\mathrm{n})$ be a mass-conserving collision operator. Then for $\mathrm{n} \in \mathcal{E}$, $\|I+\mathcal{D C}(\mathbf{n})\|_{\ell_{1}}=1$

Proof of lemma. Well, let

$$
G \equiv\left[C_{k, 1}\right]
$$

where

$$
G_{k, l}=\frac{\partial}{\partial n_{1}} H_{k}(\mathrm{n})
$$

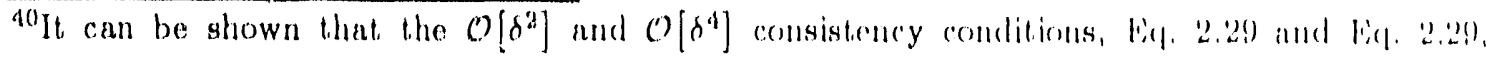
respectively, have regular solutions so that there exist miform bounds on $h^{(1)}, h^{(?)}$, and $h^{(: 3)}$.

"See Definition 2.42 for the defhnition of stability.
} 
and $I /(n)=n+C \cdot(n)$. Note that $C_{k .1} \geq 0$ for $n \in \mathcal{E}$ by Definition 2.40. Then we hisice

$$
\begin{aligned}
\|I+D \mathcal{C}(\mathbf{n})\|_{e_{1}} & =\sup _{\mid \in\{0,1, \ldots, d-1 \mid} \sum_{k=0}^{d-1}\left|C_{k, 1}\right| \\
& =\sup _{1} \sum_{k=0}^{d-1} \frac{\partial}{\partial n_{1}} H_{k}(\mathbf{n}) \\
& =\sup _{1} \frac{\partial}{\partial n_{1}} \sum_{k} H_{k}(\mathbf{n}) \\
& =\sup _{1} \frac{\partial}{\partial n_{1}} \sum_{k}\left(n_{k}+\mathcal{C}_{k}(\mathbf{n})\right) \\
& =\sup _{1}\left[1+\frac{\partial}{\partial n_{1}} \sum_{k} \mathcal{C}_{k}(\mathbf{n})\right] \\
& =1,
\end{aligned}
$$

since $\sum_{k} \mathcal{C}_{k}(\mathrm{n})=0$, i.e., $\mathcal{C}(\mathrm{n})$ is mass-conserving.

Proof of Theorem 2.58. We will show that $\left\|L\left[\mathrm{n}^{n}, \mathrm{~h}^{n}\right]\right\|_{\ell_{1}}=1 \forall n$. Since $\mathrm{n}_{i}^{0} \in \mathcal{E}$, 'Theorem 2.49 guarantees that $n_{i}^{n} \in \mathcal{E}$. Also, $h_{i}^{n} \in \mathcal{E}$ by the statement of the theorem. Then noting that $\left(n_{r}^{n}-a\left(h_{r}^{n}-n_{i}^{n}\right)\right) \in \mathcal{E}$ frr $s \in[0,1]$ so that Lemma 2.59 applies, we have

$$
\begin{aligned}
\left\|L\left[\mathrm{n}^{n}, \mathrm{~h}^{n}\right]\right\|_{\rho_{1}} & =\left\|\int_{0}^{1}\left[I+\mathcal{D C}\left(\mathrm{n}_{\tilde{r}}^{n}-s\left(\mathrm{~h}_{\tilde{r}}^{n}-\mathrm{n}_{\tilde{r}}^{n}\right)\right)\right] d s\right\|_{\ell_{1}} \\
& \leq \int_{0}^{1}\left\|I+\mathcal{D C}\left(\mathrm{n}_{\tilde{r}}^{n}-s\left(\mathrm{~h}_{\mathrm{r}}^{n}-\mathrm{n}_{\tilde{r}}^{n}\right)\right)\right\|_{\ell_{1}} d s \\
& =\int_{0}^{1} 1 d s=1 .
\end{aligned}
$$

Example ( $L B_{1}:$ Stability).

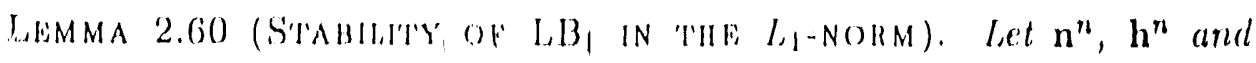
$L\left[\mathrm{n}^{n}, \mathrm{~h}^{n}\right]$ be defined as in Defintion 2.41 . Let domnain of monotonicity, $\mathcal{E}$, be 


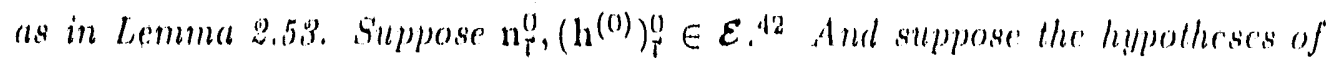
Lemma 2.56 are satisfied. Then $\exists \tau>0$ such that $\left\|l\left[\mathrm{n}^{n}, \mathrm{~h}^{n}\right]\right\|_{\ell_{1}} \leq \mid \forall n$ such that $0 \leq n \Delta t \leq T^{\prime}$ (ant $)<\Delta t<\tau$.

Proof. Let $R_{+}=M_{+}$and $R_{-}=M_{-1}$, where $M_{+}$and $M_{-.}$are defined in Lemma 2.53. Let $\delta_{0}$ be given by Lemma 2.56. Choose $r=T^{\prime}\left(\delta_{0}\right)^{2}$ so that $\Delta x \leq L \delta_{0}$. Note that Corollary 2.57 applies to yield that $\mathrm{h}^{n} \in \mathcal{E} \vee n$ such that $0 \leq n \Delta t \leq T^{\prime}$ and $0<\Delta t<\tau$. Then $\left\|l\left[\mathrm{n}^{n}, \mathrm{~h}^{n}\right]\right\|_{\ell_{1}} \leq 1$ by Lemma 2.59 .

\subsubsection{Convergence}

Convergence of a latice method can be made in terms of consistency and siability.

TuEOREM 2.61 (CONVERGENCE). Suppose a lattice method is consistent. Then stability is a sufficient condition for convergence.

Proof. We show that a consistent and stable lattice mothod is necessarily convergent. Let error $e_{i}^{n}=n_{i}^{n}-h_{\tau}^{n}$. Then note that by the Fundamental Theorem of Calculus

$$
\mathcal{A} \mathbf{e}^{n}=L\left[\mathrm{n}^{n}, \mathrm{~h}^{n}\right] \mathbf{e}^{n}+\mathcal{T}\left(\mathrm{h}_{\mathfrak{r}^{n}}^{n}\right)
$$

where

$$
L\left[\mathrm{n}^{n}, \mathrm{~h}^{n}\right]=\int_{0}^{1}\left[1+L C C\left(\mathrm{n}^{n}-\ldots \cdot s\left(\mathrm{~h}^{n}-\mathrm{n}^{n}\right)\right)\right] d s
$$

(I is the identity matrix). Then

$$
L\left[\mathbf{n}^{n}, \mathrm{~h}^{n}\right]=\operatorname{diag}\left(L_{\vec{i}}\right)_{\vec{R} \in \mathcal{L}},{ }^{43}
$$

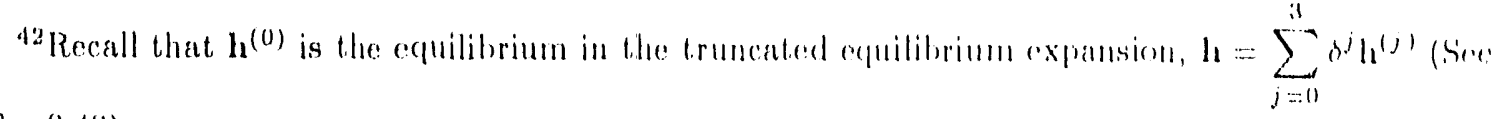
F(q. 2.42)

${ }^{43} L\left[\mathbf{n}^{\prime \prime}, h^{\prime \prime}\right]$ is block diagonal matrix. 
Whare $L_{\text {p }}$ is $n d \times d$ matrix given by

$$
L_{i}=\int_{0}^{1}\left[I+\mathcal{D C}\left(n_{\tilde{r}}^{n}+s\left(h_{r}^{n}-n_{r}^{n}\right)\right)\right] d s .
$$

Indered

$$
.4 \mathbf{e}_{\tilde{\imath}}^{n}=L_{i} \mathbf{e}_{\tau}^{n}+T\left(h_{\imath}^{n}\right)
$$

There exists a permutation matrix, $P$, such that

$$
\mathbf{e}^{n+1}=\hat{L}\left[\mathbf{n}^{n}, \mathbf{h}^{n}\right] \mathbf{e}^{n}+\hat{\mathcal{T}}\left(\mathbf{h}^{n}\right)
$$

where $(N d) \times(N d)$ matrix ${ }^{44}$,

$$
\hat{L}\left[\mathrm{n}^{n}, \mathbf{h}^{n}\right]=P^{T} L\left[\mathbf{n}^{n}, \mathrm{~h}^{n}\right] P
$$

and $\hat{\mathcal{T}}\left(\mathbf{h}^{n}\right)=P^{T} \mathcal{T}\left(\mathbf{h}^{n}\right)$.

By definition of consistency there exists a constant, $C_{0}$, such that $\left\|\mathcal{T}\left(\mathrm{h}^{n}\right)\right\| \leq$ $C_{0} \delta^{2+K}$, for some $K>0, \forall n$ such that $0 \leq n \Delta t \leq T$. By definition of stability there exists $\tau>0$ and $C_{1}$

$$
\left\|\prod_{m=0}^{n} L\left[\mathbf{n}^{m}, \mathbf{h}^{m}\right]\right\| \leq C_{1}^{\prime},
$$

for all $n$ and $\Delta t$ such that $0<\Delta t<\tau$ and $0 \leq n \Delta t \leq T$.

We have

$$
\begin{aligned}
\left\|\mathbf{e}^{n+1}\right\| & =\left\|\sum_{m=0}^{n-1}\left(\prod_{r=m+1}^{n-1} \hat{L}\left[\mathrm{n}^{r}, \mathrm{~h}^{r}\right]\right) \hat{\mathcal{T}}\left(\mathrm{h}^{m}\right)\right\| \\
& \leq\left\|P^{T}\right\| C_{1} \sum_{m=0}^{n-1}\left\|\mathcal{T}\left(\mathrm{h}^{m}\right)\right\|+\left\|P^{T}\right\|\left\|\mathcal{T}\left(\mathrm{h}^{n}\right)\right\| \\
& \leq(n-1)\left\|P^{T}\right\| C_{0} C_{1} \delta^{2+k}+\left\|P^{T}\right\| C_{0} \delta^{2+K} \\
& \leq n C \delta^{2+K},
\end{aligned}
$$

\footnotetext{
${ }^{4 A} N=\mathcal{O}\left[1 /(\Delta x)^{\prime \prime}\right]$ is the total mumber lattice notes in the lattice, $\mathbb{L}$.
} 
where $C=\left\|P^{T}\right\| \max \left(C_{0} C_{1}, C_{1}\right)$. Then

Let $\mathrm{f}_{i}^{n}$ and $F^{n}$ be as in Definition 2.44. Then $\mathbf{f}_{\tilde{i}}^{n}=\frac{1}{d} \mathbf{Q}^{T} \mathbf{e}_{\vec{i}}^{n}$ by Lemma 2.45 . Further,

$$
\left\|F^{n}\right\| \leq \frac{1}{d}\left\|Q^{T}\right\|\left\|\mathbf{e}^{n}\right\|
$$

Hence,

$$
\begin{aligned}
\frac{1}{\Delta t}\left\|F^{n+1}\right\| & \leq \frac{1}{d \Delta t}\left\|Q^{T}\right\| n\left\|\mathrm{e}^{n}\right\| \\
& \leq \frac{1}{d \Delta t}\left\|Q^{T}\right\| n C \delta^{2+K} \\
& \leq \frac{1}{d}\left\|Q^{T}\right\| n C T \delta^{K} \\
& \rightarrow 0
\end{aligned}
$$

as $\Delta t \rightarrow 0$.

Example ( $L B_{1}$ : Convergence).

Theorem 2.62 (Convergence of LB $_{1}$ ). Let the conditions of Lemmas 2.48, 2.54, and 2.56 be satisfied. Then $\lim _{\Delta t \rightarrow 0} \frac{1}{\Delta t}\left\|F^{n}\right\|_{\ell_{1}}=0, \forall n \ni 0 \leq n \Delta t \leq$ $T$.

Proof. This follows directly from Theorem 2.61.

We can recover an $\mathcal{O}\left[\delta^{2}\right]$ approximation to $u(x, y ; t)$ by the covergence arguments, the definition of the truncatcd equilibrium expansion, and consistency arguments to obtain that hydrodynamical error,

$$
\begin{aligned}
\left(f_{0}\right)_{i}^{n} & =\frac{1}{4} \mathbf{q}_{0} \cdot \mathbf{e}_{i}^{n}+\mathcal{O}\left[\delta^{2}\right] \\
& =\mathbf{p}_{i}^{n}-\rho_{i}^{n}+\mathcal{O}\left[\delta^{2}\right] \\
& =\frac{1}{4} \sum_{k=0}^{3}\left(n_{k}\right)_{i}^{n}-\frac{1}{4} \sum_{k=0}^{3}\left(h_{k}\right)_{i}^{n}+\mathcal{O}\left[\delta^{2}\right]
\end{aligned}
$$




$$
\begin{aligned}
& =\frac{1}{4} \sum_{k=0}^{3}\left(n_{k}\right)_{i}^{n}-\frac{1}{4}\left(\sum_{k=0}^{3}\left(h_{k}^{(0)}\right)_{i}^{n}+\mathcal{O}\left[\delta^{2}\right]\right)+\mathcal{O}\left[\delta^{2}\right] \\
& =\frac{1}{4} \sum_{k=0}^{3}\left(n_{k}\right)_{i}^{n}-u_{i}^{n}+\mathcal{O}\left[\delta^{2}\right] \\
& =\frac{1}{4} \sum_{k=0}^{3}\left(n_{k}\right)_{i}^{n}-u(\vec{\imath} \Delta x ; n \Delta t)+\mathcal{O}\left[\delta^{2}\right] .
\end{aligned}
$$

We have shown that the hydrodynamical mode of the lattice Boltzmann method, $L B_{1}$, converges $\mathcal{O}\left[\delta^{2}\right]$ to the solution of its hydrodynamical equation, Eq. 2.29. Assumptions and statements used to obtain convergence include: (1) monotonicity principles of the lattice method, which restricts the domain of the occupation numbers, (2) boundedness of the solutions, $u$ and $\sigma^{(2)}$ (and up to their fourth spatia! derivatives), to the $\mathcal{O}\left[\delta^{2}\right]$ and $\mathcal{O}\left[\delta^{4}\right]$ consistency conditions, and (3) a maximum/minimum principle on $u$. This concludes our example.

\subsection{Conclusion}

The analysis of this chapter showed how to obtain convergence results for lattice Boltzmann methods and the Lattice Boltzmann Approximation to lattice gas methods. Convergence can be established through formal consistency and stability arguments. These arguments are similar to those employed for establishing convergence of monotone finite difference methods. Corsistency can be obtained through two applications of the discrete Chapman-Enskog expansion (one to an infinite equilibrium expansion, one to a truncated expansion). Stability can be proven by establishing maximum/minimum principles for both the lattice method and the partial differential equation, solutions of which the lattice method approximates. Restrictions on the occupation numbers may be required for maximum/minimum principles of lattice methods to yield convergence in the $L_{1}$-norm. The next chapter applies the 
theory to additional lattice methods; its sequel provides computational evidence that substantiates the theory. 


\section{Chapter 3}

\section{Applications}

This chapter presents three lattice methods and analyzes them in accordance with the ideas set forth in the preceding chapter. Two of the methods are new and have not been previously investigated.

The first lattice method is a variation of $L B_{1}$ from the previous chapter; we denote it $\mathrm{LB}_{2}{ }^{1}$ It is the subject of Section 3.1. The second la'tice method was first introduced and analyzed in [7] as a lattice gas method for the one-dimensional viscous Burgers equation,

$$
\rho_{t}+\rho \rho_{x}=\nu \rho_{x x}
$$

The method's statistical properties, i.e., correlations and covariances, were subsequently discussed in [53]. Our treatment, the subject of Section 3.2, regards it as an example of a lattice method for advection-diffusion in one dimension, i.e., as a method with $\mathcal{O}[\delta]$ biasing in the collision rules, and we detail its analysis. In Chapter 5 , the method is applied to study a domain decomposition method for lattice gas methods. Finally, Section 3.3 presents and analyzes a lattice method for the two-dimensional

\footnotetext{
${ }^{1} \mathrm{LB}_{2}$ actually denotes one of two lattice methods. The other one is obtained by exchanging particles and holes, i.e., the meaning of 0 and 1 are reversed: In the occupation numbers, $\dot{n}_{k}=0$ would denote presence and $\dot{n}_{k}=1$ would denote absence of a particle at a node.
} 
advection-diffusion equation,

$$
\rho_{t}+\rho \rho_{x}=\nu\left(\rho_{x x}+\rho_{y y}\right)
$$

While convergence is proved for the methods in Section 3.1 and Section 3.2, convergence of the third one remains to be completed and will appear in a future work.

\section{$3.1 \quad \mathrm{LB}_{2}$}

'This section presents the numerical theory' for a lattice method that is a variation of $L B_{1} . \quad\left(L B_{1}\right.$ is analyzed extensively in the previous chapter.) The new method is denoted $L B_{2}$. Under the symmetry of exchanging particles and holes, $L B_{2}$ represents one of two identical lattice methods. One of the variants of $\mathrm{LB}_{1}$ is introduced in Section 3.1.1, and the numerical theory of the variant method comprises the succeeding sections. The equilibriurn analysis is in Section 3.1.4.

In Section 3.1.5, the discrete Chapman-Enskog expansion is reveals the hydrodynamical equations for the method. The formal convergence arguments are made in Section 3.1.6.

\subsubsection{Collision Rules}

First, note the lattice, $\mathfrak{\sim}$, and velocity vectors, $\vec{e}_{k}, k \in\{0,1,2,3\}$ are the same as those for $L B_{1}$. The collision rules for $L B_{2}$ are defined to be those for $L B_{1}$ with one addition. The additional rule adds single-particle collision rule that for a node in a pre-collision state of exactly one particle, in the post-collision state the particle returns from whence it came. The resulting collision rules are exhibited in Figure 3.1 and listed in Table 3.1. 
No Particles
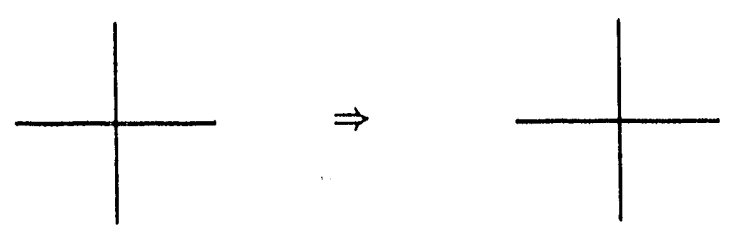

One Particle
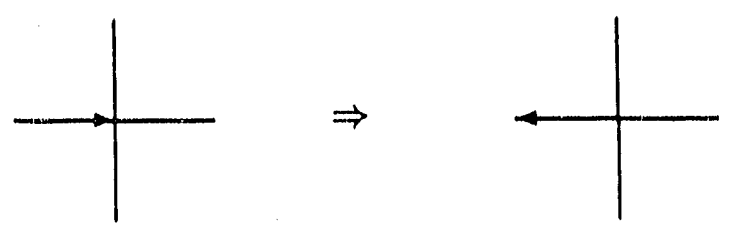

Two Orthogonal Particles
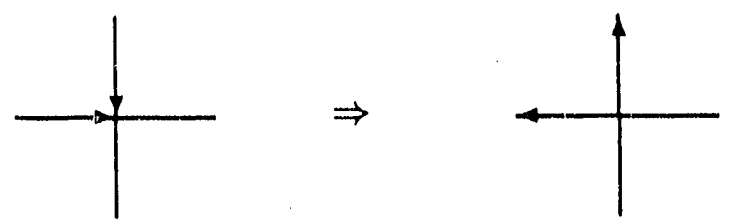

Two Head-on Particles
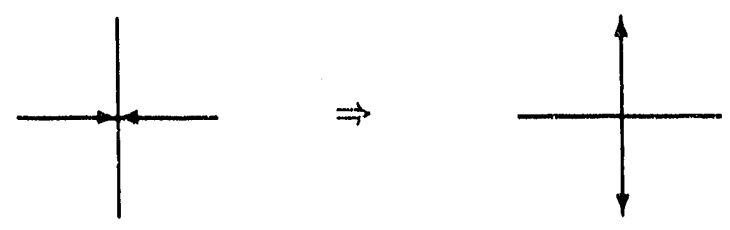

Three Particles
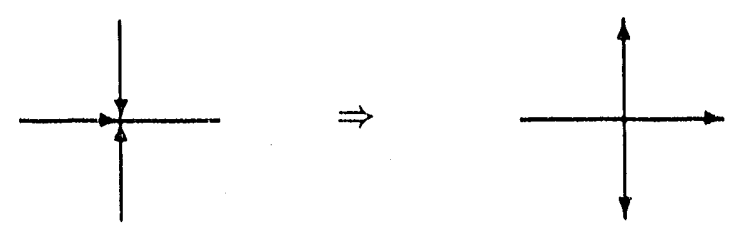

Four Particles
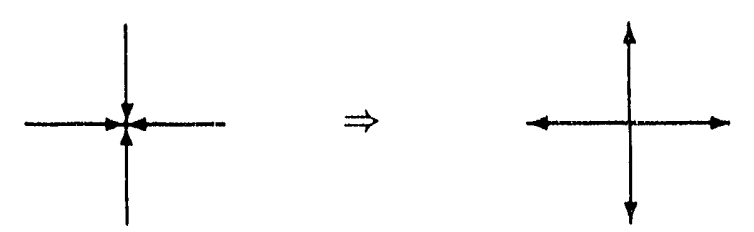

Figure 3.1: $\mathrm{LB}_{2}$ collision rules. Particles change direction only in states with exactly one or two particles present. In both the orthogonal and head-on cases of two-particle collisions, particles change direction to the moccupied directions. 
Table 3.1: $\mathrm{LB}_{2}$ Collision Rules.

\begin{tabular}{|c|c|c|c|c|c|c|c|c|c|}
\hline \multirow[t]{2}{*}{ Rule } & \multicolumn{4}{|c|}{$\alpha$} & \multicolumn{4}{|c|}{$\beta$} & \multirow{2}{*}{$\mathcal{S}(\alpha \rightarrow \beta)$} \\
\hline & $\alpha_{0}$ & $\overline{\alpha_{1}}$ & $\overline{\alpha_{2}}$ & $\overline{\alpha_{3}}$ & $\overline{\beta_{0}}$ & $\beta_{1}$ & $\beta_{2}$ & $\beta_{3}$ & \\
\hline 0 & $\overline{0}$ & 0 & 0 & 0 & $\overline{0}$ & 0 & 0 & 0 & 1 \\
\hline 1 & 0 & 0 & 0 & 1 & 0 & 1 & 0 & 0 & 1 \\
\hline 2 & 0 & 0 & 1 & 0 & 1 & 0 & 0 & 0 & 1 \\
\hline 3 & 0 & 0 & 1 & 1 & 1 & 1 & 0 & 0 & 1 \\
\hline 4 & 0 & 1 & 0 & 0 & 0 & 0 & 0 & 1 & 1 \\
\hline 5 & 0 & 1 & 0 & 1 & 1 & 0 & 1 & 0 & 1 \\
\hline 6 & 0 & 1 & 1 & 0 & 1 & 0 & 0 & 1 & 1 \\
\hline 7 & 0 & 1 & 1 & 1 & 0 & 1 & 1 & 1 & 1 \\
\hline 8 & 1 & 0 & 0 & 0 & 0 & 0 & 1 & 0 & 1 \\
\hline 9 & 1 & 0 & 0 & 1 & 0 & 1 & 1 & 0 & 1 \\
\hline 10 & 1 & 0 & 1 & 0 & 0 & 1 & 0 & 1 & 1 \\
\hline 11 & $1^{\prime}$ & 0 & 1 & 1 & 1 & 0 & 1 & 1 & 1 \\
\hline 12 & 1 & 1 & 0 & 0 & 0 & 0 & 1 & 1 & 1 \\
\hline 13 & 1 & 1 & 0 & 1 & 1 & 1 & 0 & 1 & 1 \\
\hline 14 & 1 & 1 & 1 & 0 & 1 & 1 & 1 & 0 & 1 \\
\hline 15 & 1 & 1 & 1 & 1 & 1 & 1 & 1 & 1 & 1 \\
\hline
\end{tabular}




\subsubsection{Collision Operator}

The collision operator for the lattice gas method is given by

$$
\begin{aligned}
\mathcal{C}_{k}(\hat{\mathbf{n}})= & +\overline{\hat{n}_{k}} \overline{\hat{n}}_{k+1} \hat{n}_{k+2} \hat{n}_{k+3}+\overline{\hat{n}}_{k} \hat{n}_{k+1} \overline{\hat{n}_{k+2}} \hat{n}_{k+3}+\overline{\hat{n}_{k}} \hat{n}_{k+1} \hat{n}_{k+2} \overline{\hat{n}_{k+3}} \\
& -\hat{n}_{k} \overline{\hat{n}_{k+1}} \overline{\hat{n}_{k+2}} \overline{\hat{n}_{k+3}}-\hat{n}_{k} \overline{\hat{n}_{k+1}} \overline{\hat{n}_{k+2}} \hat{n}_{k+3}-\hat{n}_{k} \overline{\hat{n}_{k+1}} \hat{n}_{k+2} \overline{\hat{n}_{k+3}} \\
& -\hat{n}_{k} \hat{n}_{k+1} \overline{\hat{n}_{k+2}} \overline{\hat{n}_{k+3}}+\hat{n}_{k} \hat{n}_{k+1} \overline{\hat{n}_{k+2}} \hat{n}_{k+3} .
\end{aligned}
$$

\subsubsection{Lattice Boltzmann Approximation}

The corresponding lattice Boltzmann method is from the Lattice Boltzmann Approximation, in which we replace the $\hat{n}_{k} \in \mathcal{B}$ with $n_{k} \in[0,1]$, where $n_{k} \approx\left\langle\hat{n}_{k}\right\rangle$. The approximation holds under "molecular chaos", in which particles are assumed to be statistically uncorrelated prior to collisions. This assumption becomes an inherent part of the lattice Boltzmann method, which then operates on mean occupation numbers, i.e., particle distributions, rather than particles themselves as with the lattice gas method.

\subsubsection{Equilibrium Analysis}

It can be easily verified that $\mathrm{LB}_{2}$ satisfies detailed balance so that the Equilibrium Theorem (Theorem 2.25) can be applied. Then considering $\mathrm{LB}_{2}$ to be in an equilibrium, i.e., $\mathcal{C}(\mathrm{n})=\mathbf{0}$, and consulting Table 3.1), the theorem yields the following: 


$$
\begin{aligned}
& \mathrm{n}^{0001}(\bar{n})^{1110}=\mathrm{n}^{0100}(\bar{n})^{1011} \quad(\text { from rules } 1 \text { and } 4) \\
& \mathrm{n}^{0010}(\overline{\mathrm{n}})^{1101}=\mathrm{n}^{1000}(\overline{\mathrm{n}})^{0111} \quad(\text { from rules } 2 \text { and } 8) \\
& \left.\mathrm{n}^{0011}(\overline{\mathrm{n}})^{1100}=\mathrm{n}^{1100}(\overline{\mathrm{n}})^{0011} \quad(\text { from rules } 3 \text { and } 12)\right\},{ }^{2} \\
& \mathrm{n}^{0110}(\overline{\mathrm{n}})^{1001}=\mathrm{n}^{1001}(\overline{\mathrm{n}})^{0110} \quad(\text { from rules } 6 \text { and } 9) \\
& \mathrm{n}^{1010}(\overline{\mathrm{n}})^{0101}=\mathrm{n}^{0101}(\overline{\mathrm{n}})^{1010} \quad(\text { from rules } 10 \text { and } 5)
\end{aligned}
$$

or

$$
\left.\begin{array}{l}
n_{3} \overline{n_{0}} \overline{n_{1}} \overline{n_{2}}=n_{1} \overline{n_{0}} \overline{n_{2}} \bar{n}_{3} \\
n_{2} \overline{n_{0}} \overline{n_{1}} \overline{n_{3}}=n_{0} \overline{n_{1}} \overline{n_{2}} \bar{n}_{3} \\
n_{2} n_{3} \overline{n_{0}} \overline{n_{1}}=n_{0} n_{1} \overline{n_{2}} \overline{n_{3}} \\
n_{1} n_{2} \overline{n_{0}} \overline{n_{3}}=n_{0} n_{3} \overline{n_{1}} \overline{n_{2}} \\
n_{0} n_{2} \overline{n_{1}} \overline{n_{3}}=n_{1} n_{3} \overline{n_{0}} \overline{n_{2}}
\end{array}\right\},
$$

for $n_{k} \in(0,1)$. Define $g_{k}=n_{k} /\left(1-n_{k}\right)$. Then Eq. 3.2 can be written

$$
\left.\begin{array}{lll}
g_{3} & =g_{1} & \text { (a) } \\
g_{2} & =g_{0} & \text { (b) } \\
g_{2} g_{3}=g_{0} g_{1} & \text { (c) } \\
g_{1} g_{2}=g_{0} g_{3} & \text { (d) } \\
g_{0} g_{2}=g_{1} g_{3} & \text { (e) }
\end{array}\right\} .
$$

Combining (d) and (e) through $g_{1}$, we get $g_{2}=g_{3}$ (because the $g_{k}$ are nonzero). Then $g_{0}=g_{1}=g_{2}=g_{3}$ and by Lemma 2.31, $n_{0}=n_{1}=n_{2}=n_{3} \equiv u$. Denoting the

\footnotetext{
${ }^{2}$ Here, the superscripts are in shorthand vector exponent notation so that, e.g.,

$$
\mathbf{n}^{0011}=\mathbf{n}\left[\begin{array}{lll}
0,1), & 1,1
\end{array}\right]^{T} .
$$
}


equilibrium solution, $n^{(0)}$, we have

$$
\mathbf{n}^{(0)}=\left[\begin{array}{l}
u \\
u \\
u \\
u
\end{array}\right]
$$

\subsubsection{Discrete Chapman-Enskog Expansion}

\section{Linearized Collision Operator}

Note that the collision operator is $\mathcal{O}[1]$. (It has no $\mathcal{O}[\delta]$ contribution for a fixed argument, i.e., $\mathcal{C}^{(1)}(\mathrm{n})=0$.) Then $\mathcal{C}(\mathrm{n})=\mathcal{C}^{(0)}(\mathbf{n})$, and we henceforth drop the superscript. The linearized collision operator is given by

$$
\mathcal{L}=(1-u)\left[\begin{array}{cccc}
-(2 u+1) & u & 1 & u \\
u & -(2 u+1) & u & 1 \\
1 & u & -(2 u+1) & u \\
u & 1 & u & -(2 u+1)
\end{array}\right]
$$

Note that indeed $\mathcal{L}$ is a symmetric circulant and thus possesses a complete orthogonal set of real eigenvectors. Note also that $\mathcal{L}$ is nonpositive definite for $u \in[0,1]$. The set of eigenpairs of the linearized collision operator, which can be found by the methods suggested on p. 52 , is

$$
\left\{\left(\lambda_{k}, \mathbf{q}_{k}\right) \mid k \in\{0,1,2,3\}\right\},
$$

where the eigenvalues are given by

$$
\begin{aligned}
& \text { eigenvalues }(\mathcal{L})=\left(\lambda_{0}, \lambda_{1}, \lambda_{2}, \lambda_{3}\right) \\
& \quad=(0,-2(1-u)(1+u),-2(1-u)(1+u),-4 u(1-u))
\end{aligned}
$$


and the respective eigenvectors (colmonn vectors) are given in the eigenmantrix,

$$
Q=\left[\begin{array}{llll}
q_{1}, & q_{1}, & q_{2}, & q_{3}
\end{array}\right]=\left[\begin{array}{rrrr}
1 & 1 & 0 & 1 \\
1 & 0 & 1 & -1 \\
1 & -1 & 0 & 1 \\
1 & 0 & -1 & -1
\end{array}\right]
$$

Note that the linearized collision operators of $L B_{1}$ and $L_{4} B_{2}$ have identical eigenvectors. Consequently, they share a common componentwise eigenvector product (*); the possible products are listed in Table 2.4 .

\section{Gradient Expressions}

Note that in the present method the unit velocity vectors, $\vec{e}_{k}(k \in\{0,1,2,3\})$, a.e the same as in $L B_{1}$. Thus, gradient expressions involving the unit velocity vectors are expressed in the same fashion (see Eq. 2.25).

\section{Matching the Advection and Collision Operator Expansions}

In matching these expansions, we assume

$$
\begin{aligned}
\Delta x & =\Delta y=L \delta \\
\Delta t & =T \delta^{2} \\
\nu & =\frac{L^{2}}{2 T}
\end{aligned}
$$

for spratial and temporal scale lengths, $L$ and $T$, respectively; $\nu$ is a diffusion coefficient. We use the following notation in the analysis:

$$
\begin{aligned}
\lambda & \equiv \lambda_{1}=\lambda_{2}=-2(1-u)(1+u), \\
D(u) & \equiv-\frac{1}{\lambda}-\frac{1}{2}
\end{aligned}
$$




$$
\begin{aligned}
D^{\prime}(u) & =\frac{d}{d u} D(u)=\frac{4 u}{\lambda^{2}}, \\
\mathbf{g}^{(j)} & =\mathcal{L} \cdot \mathbf{n}^{(j)}=\sum_{k=0}^{3} \tilde{c}_{k}^{(j)} \mathbf{q}_{k}, \\
\mathbf{n}^{(j)} & =\sigma^{(j)} \mathbf{q}_{0}+\mathcal{L}^{+} \cdot \mathbf{g}^{(j)}=\sum_{k=0}^{3} c_{k}^{(j)} \mathbf{q}_{k},
\end{aligned}
$$

in which, as with $L B_{1}, c_{0}^{(j)} \equiv \sigma^{(j)}$ and $c_{k}^{(j)}=\frac{1}{\lambda_{k}} \tilde{c}_{k}^{(j)}$ for $k \in\{1,2,3\}$. The $\sigma^{(j)}$ will be found to satisfy the consistency conditions of the lattice method. Note that $\lambda \neq \lambda_{3}$. (In referring to Eqs. 2.18 and 2.24, the reader is reminded that $\mathcal{C}^{(1)}(\mathbf{n})=0$ and $\mathcal{C}(\mathrm{n})=\mathcal{C}^{(0)}(\mathrm{n})$.)

In the convergence arguments, we will be using a third order truncated equilibrium expansion order, i.e., $\mathrm{h}=\sum_{j=0}^{3} \delta^{j} \mathbf{h}^{(j)}$, in which $\mathbf{h}^{(j)}=\mathbf{n}^{(j)}$. Since $\sigma^{(3)}$ appears in $\mathbf{n}^{(3)}$, and $\sigma^{(3)}$ is determined by the $\mathcal{O}\left[\delta^{5}\right]$ consistency condition, we carry out the discrete Chapman-Enskog procedure to the extent of determining the consistency condition at $\mathcal{O}\left[\delta^{5}\right]$. For each order in matching the advection and collision operator expansions, Eqs. 2.24 and 2.18, respectively, we find the following: ${ }^{3}$

$\mathcal{O} \mid 1]: \mathcal{C}\left(\mathbf{n}^{(0)}\right)=0$, as desired, by choice of $\mathbf{n}^{(0)}$. Then

$$
\mathbf{n}^{(0)}=\mathbf{q}_{0} u
$$

$\mathcal{O}[\delta]:$

$$
\mathcal{L} \cdot \mathbf{n}^{(1)}=\left[L\left(\vec{e}_{k} \cdot \nabla\right) n_{k}^{(0)}\right] \equiv \mathbf{g}^{(1)}=\sum_{k=0}^{3} \tilde{c}_{k}^{(1)} \mathbf{q}_{k}
$$

where

$$
\hat{c}_{0}^{(1)}=0
$$

\footnotetext{
${ }^{3}$ Since lattice methods $L B_{1}$ and $L B_{2}$ are very similar, while reading this section the reader may wish to consult the portion of Section 2.3.5 that discusses matching the advection and collision operator expansions for $L B_{1}$, pp. 72-81. Further details may found in $A$.ppendices $A$ and $B$.
} 


$$
\begin{aligned}
& i_{1}^{(1)}=L_{1} u_{x^{\prime}}, \\
& i_{2}^{(1)}=L_{1} u_{y}, \\
& \dot{c}_{2}^{(1)}=0 .
\end{aligned}
$$

- Consistency: $\mathrm{q}_{0} \cdot \mathrm{g}^{(1)}=0$ is already satisfied, so no consistency conditionis introduced at this order.

- Solve for $n^{(1)}$ : We have

$$
\begin{aligned}
n^{(1)} & =\mathcal{L}^{+} \cdot \mathrm{g}^{(1)}+\sigma^{(1)} \mathrm{q}_{0} \\
& =c_{0}^{(1)} r_{0}+\sum_{k=0}^{3} c_{k}^{(1)} \mathrm{q}_{k},
\end{aligned}
$$

where $c_{0}^{(1)}=\sigma^{(1)}$ is a free parameter that will be determined in the $\mathcal{O}\left[\delta^{3}\right]$ consistency condition, Eq. 3.8, and

$$
c_{k}^{(1)}=\frac{1}{\lambda_{k}} \dot{c}_{k}^{(1)}, k \in\{1,2,3\},
$$

or

$$
\begin{aligned}
& c_{1}^{(1)}=L \frac{1}{\lambda} u_{x}, \\
& c_{2}^{(1)}=L \frac{1}{\lambda} u_{y}, \\
& c_{3}^{(1)}=0 .
\end{aligned}
$$

$\mathcal{O}\left[\delta^{2}\right]$

$$
\begin{aligned}
\mathcal{L} \cdot \mathbf{n}^{(2)} & =\left[\frac{L^{2}}{2 !}\left(\vec{e}_{k} \cdot \nabla\right)^{2} n_{k}^{(0)}+T \frac{\partial}{\partial t} n_{k}^{(0)}+L\left(\vec{e}_{k} \cdot \nabla\right) n_{k}^{(1)}\right] \\
& -\frac{1}{2} \mathcal{D}^{2} \mathcal{C}\left(\mathbf{n}^{(0)}\right) \cdot \mathbf{n}^{(1)} \mathbf{n}^{(1)} \\
& \equiv \mathbf{g}^{(2)} \\
& =\sum_{k=0}^{3} \tilde{c}_{k}^{(2)} \mathbf{q}_{k},
\end{aligned}
$$


where

$$
\begin{aligned}
& \dot{c}_{0}^{(2)}=T u_{t}-\frac{L^{2}}{2} \nabla \cdot D(u) \nabla u, \\
& \check{c}_{1}^{(2)}=L \sigma_{x}^{(1)}-L D^{\prime}(u) \lambda \sigma^{(1)} u_{x}, \\
& \tilde{c}_{2}^{(2)}=L \sigma_{y}^{(1)}-L D^{\prime}(u) \lambda \sigma^{(1)} u_{y}, \\
& \tilde{c}_{3}^{(2)}=\frac{L^{2}}{2}\left[\frac{2 u-1}{\lambda^{2}}\left(\left(u_{t a}\right)^{2}-\left(u_{y}\right)^{2}\right)-\left(\frac{\partial}{\partial x}\left(D(u) u_{x}\right)-\frac{\partial}{\partial y}\left(D(u) u_{y}\right)\right)\right] .
\end{aligned}
$$

- Consistency: Imposing the consistency condition that $\mathbf{q}_{0} \cdot \mathbf{g}^{(2)}=0$ yields

$$
\mathbf{q}_{0} \cdot \sum_{j=0}^{3} \tilde{c}_{j}^{(2)} \mathbf{q}_{j}=4 \tilde{c}_{0}^{(2)}=0
$$

or $\tilde{c}_{0}^{(2)}=0$. With $\nu \equiv \frac{L^{2}}{2 T}$ this may be written

$$
u_{t}=\nu \nabla \cdot D(u) \nabla u
$$

- Solve for $n^{(2)}$ : We find

$$
\begin{aligned}
\mathbf{n}^{(2)} & =\mathcal{L}^{\dagger} \cdot \mathbf{g}^{(2)}+\sigma^{(2)} \mathbf{q}_{0} \\
& =\sum_{k=0}^{3} c_{k}^{(2)} \mathrm{q}_{k},
\end{aligned}
$$

where $c_{0}^{(2)}=\sigma^{(2)}$ is a free parameter that will be determined in the $\mathcal{O}\left[\delta^{4}\right]$ consistency condition), Eq. 3.14, and

$$
c_{k}^{(2)}=\frac{1}{\lambda_{k}} \tilde{c}_{k}^{(2)}, k \in\{1,2,3\},
$$

or

$$
\begin{aligned}
c_{1}^{(2)} & =\frac{L}{\lambda} \sigma_{x}^{(1)}-L D^{\prime}(u) \lambda \sigma^{(1)} u_{x} \\
c_{2}^{(2)} & =\frac{L}{\lambda} \sigma_{y}^{(1)}-L D^{\prime}(u) \lambda \sigma^{(1)} u_{y}, \\
c_{3}^{(2)} & =\frac{L^{2}}{2 \lambda_{3}}\left[\frac{2 u-1}{\lambda^{2}}\left(\left(u_{x}\right)^{2}-\left(u_{y}\right)^{2}\right)-\left(\frac{\partial}{\partial x}\left(D(u) u_{x}\right)-\frac{\partial}{\partial y}\left(D(u) u_{y}\right)\right)\right] .
\end{aligned}
$$


$\mathcal{O}\left[\delta^{3}\right]:$ We find

$$
\begin{aligned}
\mathcal{L} \cdot \mathbf{n}^{(3)}= & {\left[\frac{L^{3}}{3 !}\left(\vec{e}_{k} \cdot \nabla\right)^{3} n_{k}^{(0)}+L T\left(\vec{e}_{k} \cdot \nabla\right) \frac{\partial}{\partial t} n_{k}^{(())}+L\left(\vec{e}_{k} \cdot \nabla\right) n_{k}^{(2)}\right.} \\
& \left.+\frac{L^{2}}{2 !}\left(\vec{e}_{k} \cdot \nabla\right)^{2} n_{k}^{(1)}+T \frac{\partial}{\partial t} n_{k}^{(1)}\right] \\
- & \mathcal{D}^{2} \mathcal{C}\left(\mathbf{n}^{(0)}\right) \cdot \mathbf{n}^{(1)} \mathbf{n}^{(2)}-\frac{1}{6} \mathcal{D}^{3} \mathcal{C}\left(\mathbf{n}^{(0)}\right) \cdot \mathbf{n}^{(1)} \mathbf{n}^{(1)} \mathbf{n}^{(1)} \\
\equiv & \mathbf{g}^{(3)} \\
= & \sum_{k=0}^{3} \tilde{c}_{k}^{(3)} \mathbf{q}_{k},
\end{aligned}
$$

where

$$
\begin{aligned}
\tilde{c}_{0}^{(3)}= & T \sigma_{t}^{(1)}-\frac{I^{2}}{2} \nabla \cdot\left[D(u) \nabla \sigma^{(1)}+D^{\prime}(u) \sigma^{(1)} \nabla u\right], \\
\tilde{c}_{1}^{(3)}= & \frac{L^{3}}{6} v_{x x x}+L T u_{x t}+\left(\frac{L^{3}}{2} \frac{\partial^{2}}{\partial x^{2}}+L T \frac{\partial}{\partial t}\right)\left(\frac{1}{\lambda} u_{x}\right) \\
+ & \frac{L^{3}}{2} \frac{\partial}{\partial x}\left(\frac{1}{\lambda} u_{x}\right)-\frac{2 L}{\lambda^{3}} u_{x}\left(\left(\lambda \sigma^{(1)}\right)^{2}-\left(L u_{y}\right)^{2}\right)+L \sigma_{x}^{(2)} \\
+ & \frac{L}{2} D^{\prime}(u)\left[2\left(\lambda \sigma^{(1)}\right)^{2} D^{\prime}(u) u_{x}-2 \lambda\left(\sigma^{(2)} u_{x}+\sigma^{(1)} \sigma_{x}^{(1)}\right)\right. \\
& \left.-L^{2} u_{x}\left(\frac{\partial}{\partial x},-\frac{\partial}{\partial y}\right) \cdot D(u) \nabla u+\frac{2 L^{2}(2 u-1)}{\lambda} u_{x}\left(\left(u_{x}\right)^{2}-\left(u_{y}\right)^{2}\right)\right] \\
+ & L^{3} \frac{\partial}{\partial x}\left[\frac{1}{2 \lambda_{3}}\left(\frac{\partial}{\partial x},-\frac{\partial}{\partial y}\right) \cdot D(u) \nabla u+\frac{2 u-1}{\lambda^{2} \lambda_{3}}\left(\left(u_{x}\right)^{2}-\left(u_{y}\right)^{2}\right)\right], \\
\tilde{c}_{2}^{(3)}= & \frac{L^{3}}{6} u_{y y y}+L T u_{y t}+\left(\frac{L^{3}}{2} \frac{\partial^{2}}{\partial y^{2}}+L T \frac{\partial}{\partial t}\right)\left(\frac{1}{\lambda} u_{y}\right) \\
+ & \frac{L^{3}}{2} \frac{\partial}{\partial y}\left(\frac{1}{\lambda} u_{y}\right)-\frac{2 L}{\lambda^{3}} u_{y}\left(\left(\lambda \sigma^{(1)}\right)^{2}-\left(L u_{x}\right)^{2}\right)+L \sigma_{y}^{(2)} \\
+ & \frac{L}{2} D^{\prime}(u)\left[2\left(\lambda \sigma^{(1)}\right)^{2} D^{\prime}(u) u_{y}-2 \lambda\left(\sigma^{(2)} u_{y}+\sigma^{(1)} \sigma_{y}^{(1)}\right)\right. \\
& \left.+L^{2} u_{y}\left(\frac{\partial}{\partial x},-\frac{\partial}{\partial y}\right) \cdot D(u) \nabla u+\frac{2 L^{2}(2 u-1)}{\lambda} u_{y}\left(\left(u_{x}\right)^{2}-\left(u_{y}\right)^{2}\right)\right] \\
- & L^{3} \frac{\partial}{\partial y}\left[\frac{1}{2 \lambda_{3}}\left(\frac{\partial}{\partial x},-\frac{\partial}{\partial y}\right) \cdot D(u) \nabla u+\frac{2 u-1}{\lambda^{2} \lambda_{3}}\left(\left(u_{x}\right)^{2}-\left(u_{y}\right)^{2}\right)\right],
\end{aligned}
$$




$$
\begin{aligned}
\tilde{c}_{3}^{(3)}= & -\frac{L^{2}}{2}\left(\frac{\partial}{\partial x},-\frac{\partial}{\partial y}\right) \cdot\left[D(u) \nabla \sigma^{(1)}+D^{\prime}(\dot{u}) \sigma^{(1)} \nabla u\right]+\frac{2 L^{2}}{\lambda^{2}} \sigma^{(1)}\left(\left(u_{x}\right)^{2}-\left(u_{y}\right)^{2}\right) \\
+ & \frac{2 L^{2}(2 u-1)}{\lambda^{2}}\left[\lambda \sigma^{(1)}\left(\frac{\partial}{\partial x},-\frac{\partial}{\partial y}\right) \cdot D(u) \nabla u+\left(\sigma_{x}^{(1)} u_{x}-\sigma_{y}^{(1)} u_{y}\right)\right. \\
& \left.-\sigma^{(1)}\left((2 u-1) D^{\prime}(u) \lambda\right)\left(\left(u_{x}\right)^{2}-\left(u_{y}\right)^{2}\right)\right] .
\end{aligned}
$$

- Consistency: Imposing the consistency condition that $\mathbf{q}_{0} \cdot \mathbf{g}^{(3)}=0$, we find $\tilde{c}_{0}^{(3)}=0$, or

$$
\sigma_{t}^{(1)}=\nu \nabla \cdot\left[D(u) \nabla \sigma^{(1)}+D^{\prime}(u) \sigma^{(1)} \nabla u\right]
$$

Note that $\sigma^{(1)}(x, y ; t)=$ const. satisfies the consistency condition. Let us choose $\sigma^{(1)}(x, y ; t)=0$. This choice simplifies the $\mathcal{O}\left[\delta^{4}\right]$ calculations and is crucial for obtaining consistency and stability.

- Solve for $\mathrm{n}^{(3)}$ : We find

$$
\begin{aligned}
\mathbf{n}^{(3)} & =\mathcal{L}^{+} \cdot \mathbf{g}^{(3)}+\sigma^{(3)} \mathbf{q}_{0} \\
& =\sum_{k=0}^{3} c_{k}^{(3)} \mathbf{q}_{k}
\end{aligned}
$$

where $c_{0}^{(3)}=\sigma^{(3)}$ is a free parameter that is determined in the $\mathcal{O}\left[\delta^{5}\right]$ consistency condition, Eq. 3.16, and

$$
c_{k}^{(3)}=\frac{1}{\lambda_{k}} \check{c}_{k}^{(3)}, k \in\{1,2,3\} .
$$

Note that if $\sigma^{(1)}(x, y ; t)=0$, then $\dot{c}_{3}^{(3)}=c_{3}^{(3)}=0$. Further calculations in the derivations assume $\sigma^{(1)}(x, y ; t)=0$.

$\mathcal{O}\left[\delta^{4}\right]:$ We find

$\mathcal{L} \cdot \mathrm{n}^{(4)}$ 


$$
\begin{aligned}
= & {\left[\frac{L^{4}}{4 !} \vec{r}_{k} \cdot \nabla\right)^{4} n_{k}^{(0)}+\frac{L^{2} T}{2}\left(\vec{e}_{k} \cdot \nabla\right)^{2} \frac{\partial}{\partial t} n_{k}^{(0)}+\frac{T^{2}}{2} \frac{\partial^{2}}{\partial t^{2}} n_{k}^{(0)}+L\left(\vec{e}_{k} \cdot \nabla\right) n_{k}^{(3)} } \\
& \left.\frac{L^{2}}{2 !}\left(\vec{e}_{k} \cdot \nabla\right)^{2} n_{k}^{(2)}+T \frac{\partial}{\partial t} n_{k}^{(2)}+\frac{L^{3}}{3 !}\left(\vec{e}_{k} \cdot \nabla\right)^{3} n_{k}^{(1)}+L T\left(\vec{e}_{k} \cdot \nabla\right) \frac{\partial}{\partial t} n_{k}^{(1)}\right] \\
- & \frac{1}{24} \mathcal{D}^{4} \mathcal{C}\left(\mathbf{n}^{(0)}\right) \cdot \mathbf{n}^{(1)} \mathbf{n}^{(1)} \mathbf{n}^{(1)} \mathbf{n}^{(1)}-\frac{1}{2} \mathcal{D}^{3} \mathcal{C}\left(\mathbf{n}^{(0)}\right) \cdot \mathbf{n}^{(1)} \mathbf{n}^{(1)} \mathbf{n}^{(2)} \\
- & \mathcal{D}^{2} \mathcal{C}\left(\mathbf{n}^{(0)}\right) \cdot \mathbf{n}^{(1)} \mathbf{n}^{(3)}-\frac{1}{2} \mathcal{D}^{2} \mathcal{C}\left(\mathbf{n}^{(0)}\right) \cdot \mathbf{n}^{(2)} \mathbf{n}^{(2)} \\
\equiv & \mathbf{g}^{(4)} \\
= & \sum_{k=0}^{3} \hat{c}_{k}^{(4)} \mathbf{q}_{k},
\end{aligned}
$$

where

$$
\begin{aligned}
\tilde{c}_{0}^{(4)}= & \frac{L^{4}}{48}\left(u_{x x x x}+u_{y y y y}\right)+\frac{L^{2}}{4} \nabla^{2} u_{t}+\frac{T^{2}}{2} u_{t t}+T \sigma_{t}^{(3)} \\
+ & \frac{L^{4}}{12}\left(\frac{\partial^{3}}{\partial x^{3}}, \frac{\partial^{3}}{\partial y^{3}}\right) \cdot\left(\frac{1}{\lambda} \nabla u\right)+\frac{L^{2} T}{2} \nabla \cdot \frac{\partial}{\partial t}\left(\frac{1}{\lambda} \nabla u\right) \\
+ & \frac{L^{2}}{4}\left[\nabla^{2} \sigma^{(2)}+L^{2}\left(\frac{\partial^{2}}{\partial x^{2}}-\frac{\partial^{2}}{\partial y^{2}}\right) c_{3}^{(2)}\right]+\frac{L}{2}\left(\frac{\partial}{\partial x} c_{1}^{(3)}+\frac{\partial}{\partial y} c_{2}^{(3)}\right) \\
\tilde{c}_{1}^{(4)}= & L\left(\sigma_{x}^{(3)}-D^{\prime}(u) \lambda \sigma^{(3)} u_{x}\right), \\
\tilde{c}_{2}^{(4)}= & L\left(\sigma_{y}^{(3)}-D^{\prime}(u) \lambda \sigma^{(3)} u_{y}\right), \\
\tilde{c}_{3}^{(4)}= & \frac{L^{4}}{48}\left(u_{x x x x}-u_{y y y y}\right)+\frac{L^{2} T}{4}\left(u_{x x t}-u_{y y t}\right)+T \frac{\partial}{\partial t} c_{3}^{(2)} \\
+ & \frac{L^{2}}{4}\left[\sigma_{x x}^{(3)}-\sigma_{y y}^{(3)}+L^{2} \nabla^{2} c_{3}^{(2)}\right]+\frac{L}{2}\left(\frac{\partial}{\partial x} c_{1}^{(3)}-\frac{\partial}{\partial y} c_{2}^{(3)}\right) \\
+ & \frac{L^{4}}{12}\left(\frac{\partial^{3}}{\partial x^{3}},-\frac{\partial^{3}}{\partial y^{3}}\right) \cdot\left(\frac{1}{\lambda} \nabla u\right)+\frac{L^{2} T}{2}\left(\frac{\partial}{\partial x},-\frac{\partial}{\partial y}\right) \cdot \frac{\partial}{\partial t}\left(\frac{1}{\lambda} \nabla u\right) \\
+ & \frac{2 L(2 u-1)}{\lambda}\left(c_{1}^{(3)} u_{x}-c_{2}^{(3)}\right)-\frac{L^{2}}{\lambda^{3}}\left[\frac{2 I^{2}(2 u-1)}{\lambda \lambda_{3}}\left(\left(u_{x}\right)^{4}-\left(u_{y}\right)^{4}\right)\right. \\
& \left.-2 \lambda \sigma^{(2)}\left(\left(u_{x}\right)^{2}-\left(u_{y}\right)^{2}\right)-\frac{L^{2} \lambda}{\lambda_{3}}\left(\left(u_{x}\right)^{2}+\left(u_{y}\right)^{2}\right)\left(\frac{\partial}{\partial x},-\frac{\partial}{\partial y}\right) \cdot D(u) \nabla u\right] \\
& -\frac{2 L^{2}(2 u-1)}{\lambda_{3}} \sigma^{(2)}\left[\frac{2(2 u-1)}{\lambda^{2}}\left(\left(u_{x}\right)^{2}-\left(u_{y}\right)^{2}\right)-\left(\frac{\partial}{\partial x},-\frac{\partial}{\partial y}\right) \cdot D(u) \nabla u\right] .
\end{aligned}
$$


- Consistency: Imposing the consistency condition that $q_{0} \cdot g^{(4)}=0$, we find

$$
\begin{aligned}
\sigma_{t}^{(2)} & =\nu \nabla \cdot\left[D(u) \nabla \sigma^{(2)}+D^{\prime}(u) \sigma^{(2)} \nabla u\right] \\
& -\mathcal{F}\left(L, T ; x, y, t ; u, u_{x_{i}}, u_{x_{i} x_{j}}, u_{x_{i} x_{j} x_{k}}, u_{x_{i} x_{j} x_{k} x_{l}}\right)
\end{aligned}
$$

where $x_{0}$ and $x_{1}$ denote $x$ and $y$, respectively. Note that $\mathcal{F}$ can be explicitly expressed in terms of the spatial derivatives of $u$ using the results of this section and the details of Appendix B. (The $\mathcal{O}\left[\delta^{2}\right]$ consistency condition, Eq. 3.6, can be used to remove dependences of $\mathcal{F}$ on the temporal derivatives of $u$.)

Note that Eq. 3.14 may be written

$$
(\mathbf{L}+\mathbf{h})\left[\sigma^{(2)}\right]=\mathcal{F},
$$

where operator

$$
\mathrm{L} \equiv \sum_{i, j=0}^{1} a_{i j}(\vec{x} ; t) \frac{\partial^{2}}{\partial x_{i} \partial x_{j}}+\sum_{i=0}^{1} b_{i}(\vec{x} ; t) \frac{\partial}{\partial x_{i}}-\frac{\partial}{\partial t},
$$

in which

$$
\begin{aligned}
& a_{00}(x, y ; t)=\nu D(u), \\
& a_{01}(x, y ; t)=0 \\
& a_{10}(x, y ; t)=0, \\
& a_{11}(x, y ; t)=\nu D(u) ; \\
& b_{0}(x, y ; t)=\nu D^{\prime}(u) u_{x}, \\
& b_{1}(x, y ; t)=\nu D^{\prime}\left(u ; u_{y} ;\right.
\end{aligned}
$$

and

$$
\mathbf{h}(x, y ; t)=\nu \nabla \cdot D^{\prime}(u) \nabla u .
$$


Note that operator $\mathrm{L}$ is uniformly parabolic in $E_{T}=([0, L] \times[0, L]) \times(0, T]$ for $u \in C^{1}\left([0, L]^{2},(0,1)\right)$ Suppose further that $u \in C^{4}\left(\mathcal{R}^{2}, \mathcal{R}\right)$. Then under these and additional assumptions (regarding smoothnes:; and initial and boundary conditions) and regularty arguments, it can be shown [54] that solutions $\sigma^{(2)}$ of Eq. 3.15 are $C^{2}\left(\mathcal{R}^{2}, \mathcal{R}\right)$ functions. Then bounds on $\sigma^{(2)}$ and up to its second derivatives exist. We will assume such bounds to obtain statements regarding numerical convergence of the lattice method. (See Lemma 3.1 for how the bounds apply to numerical consistency and Lemma 3.5 for how they apply to continuum maximum and minimum principles for the truncated equilibrium expansion.)

- Solve for $n^{(4)}$ : We find

$$
\mathbf{n}^{(4)}=\mathcal{L}^{+} \cdot \mathbf{g}^{(4)}+\sigma^{(4)} \mathbf{q}_{0}=\sum_{k=0}^{3} c_{k}^{(4)} \mathbf{q}_{k},
$$

where $c_{0}^{(4)}=\sigma^{(4)}$ is a free parameter that would be determined in the $\mathcal{O}\left[\delta^{6}\right]$ consistency condition, and $c_{k}^{(4)}=\frac{1}{\lambda_{k}} \tilde{c}_{k}^{(4)}, k \in\{1,2,3\}$.

$\mathcal{O}\left[\delta^{5}\right]$ : At this order, only the consistency condition is to be determined. This condition specifies $\sigma^{(3)}$. We will find that any $\sigma^{(3)}(x, y ; t)=$ const. satisfies this condition. We have

$$
\begin{aligned}
\mathcal{L} \cdot \mathbf{n}^{(5)} & \\
= & {\left[\frac{L^{5}}{5 !}\left(\vec{e}_{k} \cdot \nabla\right)^{5} n_{k}^{(0)}+\frac{L T^{2}}{2}\left(\vec{e}_{k} \cdot \nabla\right) \frac{\partial^{2}}{\partial t^{2}} n_{k}^{(0)}+\frac{L^{3}}{6}\left(\vec{e}_{k} \cdot \nabla\right)^{3} \frac{\partial}{\partial t} n_{k}^{(0)}\right.} \\
& +\frac{L^{4}}{4 !}\left(\vec{e}_{k} \cdot \nabla\right)^{4} n_{k}^{(1)}+\frac{L^{2} T}{2}\left(\vec{e}_{k} \cdot \nabla\right)^{2} \frac{\partial}{\partial t} n_{k}^{(1)}+\frac{T^{2}}{2} \frac{\partial^{2}}{\partial t^{2}} n_{k}^{(1)} \\
& \left.+\frac{L^{3}}{3 !}\left(\vec{e}_{k} \cdot \nabla\right)^{3} n_{k}^{(2)}+L T\left(\vec{e}_{k} \cdot \nabla\right) \frac{\partial}{\partial t} n_{k}^{(2)}+\frac{L^{2}}{2 !}\left(\vec{e}_{k} \cdot \nabla\right)^{2} n_{k}^{(3)}+L\left(\vec{e}_{k} \cdot \nabla\right) n_{k}^{(4)}\right]
\end{aligned}
$$




$$
\begin{aligned}
& + \text { (Coll.) } \\
& =\sum_{k=0}^{3} \tilde{c}_{k}^{(5)} \mathbf{q}_{k},
\end{aligned}
$$

where (Coll.) is the contribution from the coefficient of $\delta^{5}$ in the collision operator expansion, Eq. 2.18. Since (Coll.) is orthogonal to the nullspace of the linearized collision operator, it does not contribute to the consistency condition. Note that $\tilde{c}_{0}^{(5)}$ is completely determined in view of this by the terms listed in Appendix B. The remaining coefficients, $\tilde{c}_{k}^{(5)}(k \in\{1,2,3\})$, are left uncalculated. ${ }^{4}$

- Consistency: Imposing the consistency condition that $q_{0} \cdot g^{(5)}=0$ implies $\tilde{c}_{0}^{(5)}=0$, or

$$
\sigma_{t}^{(3)}=\nu \nabla \cdot\left[D(u) \nabla \sigma^{(3)}+D^{\prime}(u) \sigma^{(3)} \nabla u\right] .
$$

Note that any constant $\sigma^{(3)}(x, y ; t)$ satisfies the consistency condition. Then choosing $\sigma^{(3)}(x, y ; t)=0$ implies that $\tilde{c}_{1}^{(4)}=\tilde{c}_{2}^{(4)}=0$.

- Solve for $\mathrm{n}^{(5)}$ : We have no need for calculating this quantity since the $\mathcal{O}\left[\delta^{6}\right]$ consistency condition is not desired.

\subsubsection{Convergence}

LEMma 3.1 (CONSISTENCY of $\mathrm{LB}_{2}$ ). Define the truncated equilibrium expansion by

$$
\mathrm{h}=\sum_{j=0}^{3} \delta^{j} \mathbf{h}^{(j)}
$$

\footnotetext{
${ }^{4}$ The $\tilde{c}_{k}^{(5)}(k \in\{1,2,3\})$ would need to be calculated for determining $\mathbf{n}^{(5)}$, which mean also calculating the (Coll.) term. One would complete such calculations to determine the next order's consistency condition.
} 
in which $\mathrm{h}^{(j)} \equiv \mathrm{n}^{(j)}$, the $\mathrm{n}^{(j)}$ as defincd by Eqs. 3.4, 3.5. 3.7. and 3.9 in the application of the discrete Chapman-Enstog procedure of Section 3.1.5. Then h satisfies

$$
\mathcal{A} \mathrm{h}=\mathrm{h}+\mathcal{C}(\mathrm{h})-\mathcal{T}(\mathrm{h})
$$

in which $\mathcal{T}(\mathbf{h})=\mathcal{O}\left[\delta^{4}\right]$. Suppose $\sigma^{(0)} \in C^{4}\left(\mathcal{R}^{2}, \mathcal{R}\right)$ and $\sigma^{(2)} \in C^{2}\left(\mathcal{R}^{2}, \mathcal{R}\right)$. Let $\Delta t=T \delta^{2}$, and $\Delta x=L \delta$ for spatidi and temporal scale lengths $L$ and $T$, respectively. Then

$$
\lim _{\Delta t \rightarrow 0} \frac{1}{\Delta t}\|\mathcal{T}(\mathbf{h})\|=0
$$

for some norm, $\|\cdot\|$.

Proof. First, we determine the parameters of the truncated equilibrium expansion in terms of the expansion for $\mathbf{n}$. Then we determine the remainder, $\mathcal{T}(\mathbf{h})$. Finally, we show that $\lim _{\Delta t \rightarrow 0} \frac{1}{\Delta t}\|\mathcal{T}(\mathrm{h})\|=0$, for some norm $\|\cdot\|$.

\section{Part 1: Determining the Truncated Equilibrium Expansion}

Recall that any constant $\sigma^{(1)}(x, y ; t)$ and $\sigma^{(3)}(x, y ; t)$ satisfy the $\mathcal{O}\left[\delta^{3}\right]$ and $\mathcal{O}\left[\delta^{5}\right]$ consistency conditions, i.e., Eq. 3.8 and Eq. 3.16, respectively. Choose $\sigma^{(1)}(x, y ; t)=$ $\sigma^{(3)}(x, y ; t)=0$. Then using that $\mathrm{h}^{(j)} \equiv \mathrm{n}^{(j)}$ and Eqs. 3.4, 3.5, 3.7, and 3.9 for $\mathrm{n}^{\left(0^{\prime}\right.}$ $n^{(1)}, n^{(2)}$, and $n^{(3)}$, respectively, we find

$$
\begin{gathered}
\mathrm{h}^{(0)}=\mathrm{q}_{0} u . \\
\mathrm{h}^{(1)}=\sum_{k=0}^{3} c_{k}^{(1)} \mathrm{q}_{k},
\end{gathered}
$$

where

$$
\begin{aligned}
& c_{0}^{(1)}=0, \\
& c_{1}^{(1)}=\frac{L}{\lambda} u_{x},
\end{aligned}
$$




$$
\begin{aligned}
c_{2}^{(1)} & =\frac{L}{\lambda} u_{y}, \\
c_{3}^{(1)} & =0 \\
\mathbf{h}^{(2)} & =\sum_{k=0}^{3} c_{k}^{(2)} \mathbf{q}_{k},
\end{aligned}
$$

where

$$
\begin{aligned}
& c_{0}^{(2)}=\sigma^{(2)} \\
& c_{1}^{(2)}=0, \\
& c_{2}^{(2)}=0, \\
& c_{3}^{(2)}=\frac{L^{2}}{2 \lambda_{3}}\left[\frac{2 L^{2}(2 u-1)}{\lambda^{2}}\left(\left(u_{x}\right)^{2}-\left(u_{y}\right)^{2}\right)-2\left(\frac{\partial}{\partial x}\left(D(u) u_{x}\right)-\frac{\partial}{\partial y}\left(D(u) u_{y}\right)\right)\right] ;
\end{aligned}
$$

and

$$
\mathbf{h}^{(3)}=\sum_{k=0}^{3} c_{k}^{(3)} \mathbf{q}_{k}
$$

where

$$
\begin{aligned}
c_{0}^{(3)} & =0 \\
c_{1}^{(3)} & =\frac{1}{\lambda}\left[\frac{L^{3}}{6} u_{x x x}+L T u_{x t}+L T \frac{\partial}{\partial t}\left(\frac{1}{\lambda} u_{x}\right)\right. \\
& +L \sigma_{x}^{(2)}+\frac{2 L^{3}}{\lambda^{3}} u_{x}\left(u_{y}\right)^{2}-L D^{\prime}(u) \lambda \sigma^{(2)} u_{x} \\
& +\frac{L}{2} D^{\prime}(u)\left[\frac{2 L^{2}(2 u-1)}{\lambda} u_{x}\left(\left(u_{x}\right)^{2}-\left(u_{y}\right)^{2}\right)-L^{2} u_{x}\left(\frac{\partial}{\partial x},-\frac{\partial}{\partial y}\right) \cdot D(u) \nabla u\right] \\
& \left.+L^{3} \frac{\partial}{\partial x}\left[\frac{1}{2 \lambda_{3}}\left(\frac{\partial}{\partial x},-\frac{\partial}{\partial y}\right) \cdot D(u) \nabla u+\frac{2 u-1}{\lambda^{2} \lambda_{3}}\left(\left(u_{x}\right)^{2}-\left(u_{y}\right)^{2}\right)\right]\right], \\
c_{2}^{(3)} & =\frac{1}{\lambda}\left[\frac{L^{3}}{6} u_{y y y}+L T u_{y t}+L T \frac{\partial}{\partial t}\left(\frac{1}{\lambda} u_{y}\right)\right. \\
& +L \sigma_{y}^{(2)}-\frac{2 L^{3}}{\lambda^{3}}\left(u_{x}\right)^{2} u_{y}-L D^{\prime}(u) \lambda \sigma^{(2)} u_{y} \\
& +\frac{L}{2} D^{\prime}(u)\left[-\frac{2 L^{2}(2 u-1)}{\lambda} u_{y}\left(\left(u_{x}\right)^{2}-\left(u_{y}\right)^{2}\right)+L^{2} u_{y}\left(\frac{\partial}{\partial x},-\frac{\partial}{\partial y}\right) \cdot D(u) \nabla u\right]
\end{aligned}
$$




$$
\begin{aligned}
& \left.-L^{3} \frac{\partial}{\partial y}\left[\frac{1}{2 \lambda_{3}}\left(\frac{\partial}{\partial x},-\frac{\partial}{\partial y}\right) \cdot D(u) \nabla u+\frac{2 u-1}{\lambda^{2} \lambda_{3}}\left(\left(u_{x}\right)^{2}-\left(u_{y}\right)^{2}\right)\right]\right], \\
c_{3}^{(3)}= & 0 .
\end{aligned}
$$

Part 2: Determining the Remainder

Now, define truncation error, $\mathcal{T}(\mathrm{h})$, by

$$
\mathcal{A} \mathbf{h}-\mathbf{h}-\mathcal{C}(\mathbf{h})=-\mathcal{T}(\mathbf{h})=-\sum_{j=0}^{4} \mathrm{~T}^{(j)} \delta^{j}+\mathcal{O}\left[\delta^{5}\right],
$$

where

$$
\begin{aligned}
\mathbf{T}^{(0)}= & \mathcal{C}\left(\mathbf{h}^{(0)}\right) \\
\mathbf{T}^{(1)}= & \mathcal{L} \cdot \mathbf{h}^{(1)}-\left[L\left(\vec{e}_{k} \cdot \nabla\right) h_{k}^{(0)}\right], \\
\mathbf{T}^{(2)}= & \mathcal{L} \cdot \mathbf{h}^{(2)}+\frac{1}{2} \mathcal{D}^{2} \mathcal{C}\left(\mathbf{h}^{(0)}\right) \cdot \mathbf{h}^{(1)} \mathbf{h}^{(1)} \\
- & {\left[\frac{L^{2}}{2 !}\left(\vec{e}_{k} \cdot \nabla\right)^{2} h_{k}^{(0)}+T \frac{\partial}{\partial t} h_{k}^{(0)}+L\left(\vec{e}_{k} \cdot \nabla\right) h_{k}^{(1)}\right], } \\
\mathbf{T}^{(3)}= & \mathcal{L} \cdot \mathbf{h}^{(3)}+\frac{1}{6} \mathcal{D}^{3} \mathcal{C}\left(\mathbf{h}^{(0)}\right) \cdot \mathbf{h}^{(1)} \mathbf{h}^{(1)} \mathbf{h}^{(1)}+\mathcal{D}^{2} \mathcal{C}\left(\mathbf{h}^{(0)}\right) \cdot \mathbf{h}^{(1)} \mathbf{h}^{(2)} \\
- & {\left[\frac{L^{3}}{3 !}\left(\vec{e}_{k} \cdot \nabla\right)^{3} h_{k}^{(0)}+L T\left(\vec{e}_{k} \cdot \nabla\right) \frac{\partial}{\partial t} h_{k}^{(0)}+\frac{L^{2}}{2 !}\left(\vec{e}_{k} \cdot \nabla\right)^{2} h_{k}^{(1)}\right.} \\
& \left.+T \frac{\partial}{\partial t} h_{k}^{(1)}+L\left(\vec{e}_{k} \cdot \nabla\right) n_{k}^{(2)}\right], \\
\mathbf{T}^{(4)}= & \frac{1}{24} \mathcal{D}^{4} \mathcal{C}\left(\mathbf{h}^{(0)}\right) \cdot \mathbf{h}^{(1)} \mathbf{h}^{(1)} \mathbf{h}^{(1)} \mathbf{h}^{(1)}+\frac{1}{2} \mathcal{D}^{3} \mathcal{C}\left(\mathbf{h}^{(0)}\right) \cdot \mathbf{h}^{(1)} \mathbf{h}^{(1)} \mathbf{h}^{(2)} \\
+ & \frac{1}{2} \mathcal{D}^{2} \mathcal{C}\left(\mathbf{h}^{(0)}\right) \cdot \mathbf{h}^{(2)} \mathbf{h}^{(2)}+\mathcal{D}^{2} \mathcal{C}\left(\mathbf{h}^{(0)}\right) \cdot \mathbf{h}^{(1)} \mathbf{h}^{(3)} \\
- & {\left[\frac{L^{4}}{4 !}\left(\vec{e}_{k} \cdot \nabla\right)^{4} h_{k}^{(0)}+\frac{L^{2} T}{2}\left(\vec{e}_{k} \cdot \nabla\right)^{2} \frac{\partial}{\partial t} h_{k}^{(0)}+\frac{T^{2}}{2} \frac{\partial^{2}}{\partial t^{2}} h_{k}^{(0)}+L\left(\vec{e}_{k} \cdot \nabla\right) h_{k}^{(3)}\right.} \\
& \left.+\frac{L^{2}}{2 !}\left(\vec{e}_{k} \cdot \nabla\right)^{2} h_{k}^{(2)}+T \frac{\partial}{\partial t} h_{k}^{(2)}+\frac{L^{3}}{3 !}\left(\vec{e}_{k} \cdot \nabla 7\right)^{3} h_{k}^{(1)}+L T\left(\vec{e}_{k} \cdot \nabla\right) \frac{\partial}{\partial t} h_{k}^{(1)}\right] .
\end{aligned}
$$


By the discrete Chapman-Enskog expansion on h, we find

$$
\mathrm{T}^{(0)}=\mathrm{T}^{(1)}=\mathrm{T}^{(2)}=\mathrm{T}^{(3)}=0
$$

with our choice of $\sigma^{(1)}(x, y ; t)=\sigma^{(3)}(x, y ; t)=0$. Further, we find

$$
\mathbf{T}^{(4)}=\sum_{k=0}^{3} \mathbf{T}_{k}^{(4)} \mathbf{q}_{k}
$$

where

$$
\begin{aligned}
& \mathrm{T}_{0}^{(4)}=\tilde{c}_{0}^{(4)}=0,\left(\text { because of the } \mathcal{O}\left[\delta^{4}\right]\right. \text { consistency condition) } \\
& \mathrm{T}_{1}^{(4)}=\tilde{c}_{1}^{(4)}=0,\left(\text { because } \sigma^{(3)}=0\right) \\
& \mathrm{T}_{2}^{(4)}=\tilde{c}_{2}^{(4)}=0,\left(\text { because } \sigma^{(3)}=0\right) \\
& \mathrm{T}_{3}^{(4)}=\left.\tilde{c}_{3}^{(4)}\right|_{\sigma^{(1)}(x, y ; t)=\sigma^{(3)}(x, y ; t)=0}
\end{aligned}
$$

in which the $\tilde{c}_{k}^{(4)}$ are given in Eqs. 3.10-3.13. Note that $\mathbf{T}_{3}^{(4)}$ is a functional in $u$ and up to its fourth spatial partial derivatives, and in $\sigma^{(2)}$ and up to its second spatial partial derivatives.

\section{Part 3: Showing Consistency}

The combined results of parts 1 and 2 establish consistency, as summarized by the following: Note that the $\mathrm{h}^{(j)}, j \in\{0,1,2,3\}$, are uniformly bounded since

$$
\sigma^{(0)} \in C^{4}\left(\mathcal{R}^{2}, \mathcal{R}\right) \text { and } \sigma^{(2)} \in C^{2}\left(\mathcal{R}^{2}, \mathcal{R}\right)
$$

by the assumptions of the lemma. Hence, by Theorem $2.47 \exists$ a norm, $\|\cdot\|$, such that $\lim _{\Delta t \rightarrow 0} \frac{1}{\Delta t}\|\mathcal{T}(\mathbf{h})\|=0$.

Lemma 3.2 (Domain of Monotonicity for $L_{B}$ ). Let $\mathcal{E}^{(k)}=\left[M_{-}, M_{+}\right]$for $k \in\{0,1,2,3\}$, where $M_{-}=2 / 3$ and $M_{+}=5 / 6$. Then

$$
\mathcal{E}=\mathcal{E}^{(0)} \times \mathcal{E}^{(1)} \times \mathcal{E}^{(2)} \times \mathcal{E}^{(3)}
$$


is the domain of monotonicity for the lattice method, L,B..

(Since the proof is rather long and involved, the reader may wish to accept the lemma and proceed to the discussion and development of the continumm maximum and minimum principles begiming on p. 150.)

Proof. There are three parts to proving the lomma: (1) show that the conditions of monotonicity are satisfied in $\mathcal{E}$, (2) show that $\mathcal{E}$ cannot be extended to a larger connected region, and (3) show that $\mathcal{E}$ has volume. We will proceed in the order (3), (1). alld (2)

(3) Clearly, $\mathcal{E}$ has volume since $M_{-}<M_{+}$.

(1) Our proof is by induction on $n$. Note that the base step is a special case of the induction step. The induction hypothesis is to show that the conditions of monotonicity are satisfied in $\mathcal{E}$, we begin by rewriting the Lattice Boltzmann Equation for $\mathrm{LB}_{2}$ in terms of $n^{n+1}$ to get

$$
n_{i}^{n+1}=\left[\left(n_{k}\right)_{i-\tilde{e}_{k}}^{n}+\mathcal{C}_{k}\left(n_{i-\tilde{e}_{k}}^{n}\right)\right]_{k=0}^{k=3}
$$

where $\vec{\imath}=(i, j)$ and $\vec{e}_{k}$ is the unit velocity vector for direction $k$. Then $H$ is defined by

$$
H\left(\left[\mathrm{n}_{\boldsymbol{\Gamma}-\tilde{e}_{k}}\right]_{k=0}^{k=3}\right) \equiv\left[H_{k}\left(\mathbf{n}_{\boldsymbol{\top}-\tilde{e}_{k}}^{n}\right)\right]_{k=0}^{k=3},
$$

where

$$
H_{k}\left(\mathbf{n}_{\tilde{i}-\tilde{e}_{k}}^{n}\right) \equiv\left(n_{k}\right)_{i-\tilde{e}_{k}}^{n}+\mathcal{C}_{k}\left(\mathbf{n}_{\tilde{i}-\tilde{e}_{k}}^{n}\right)
$$

Then substituting for the $\mathrm{LB}_{2}$ collision operator (Fq. 3.1), $H_{k}$ may be written explicitly,

$$
\left(n_{k}\right)_{r}^{n+1}=H_{k}\left(n_{r-\vec{e}_{k}}^{n}\right)
$$




$$
\begin{aligned}
& =\left(n_{k}\right)_{r-\delta_{k}}^{n}+\mathcal{C}_{k}\left(\mathbf{n}_{\tau-\delta_{k}}^{n}\right) \\
& =\left(n_{k}\right)_{i-e_{k}}^{n} \\
& +\overline{\left(n_{k}\right)_{i-\delta_{k}}^{n}} \overline{\left(n_{k+1}\right)_{i-\delta_{k}}^{n}}\left(n_{k+2}\right)_{t-\delta_{k}}^{n}\left(n_{k+3}\right)_{t-\delta_{k}}^{n} \\
& +\overline{\left(n_{k}\right)_{r-\delta_{k}}^{n}}\left(n_{k+1}\right)_{r-\delta_{k}}^{n} \overline{\left(n_{k+2}\right)_{r-\delta_{k}}^{n}}\left(n_{k+3}\right)_{r-\delta_{k}}^{n} \\
& +\overline{\left(n_{k}\right)_{r-\tau_{k}}^{n}}\left(n_{k+1}\right)_{r-\delta_{k}}^{n}\left(n_{k+2}\right)_{r-\delta_{k}}^{n} \overline{\left(n_{k+3}\right)_{r-\delta_{k}}^{n}} \\
& +\left(n_{k}\right)_{i-\delta_{k}}^{n} \overline{\left(n_{k+1}\right)_{i-\varepsilon_{k}}^{n}} \overline{\left(n_{k+2}\right)_{i-\varepsilon_{k}}^{n}} \overline{\left(n_{k+3}\right)_{i-\sigma_{k}}^{n}} \\
& \text { - } \overline{\left(n_{k}\right)_{i-e_{k}}^{n}}\left(n_{k+1}\right)_{i-e_{k}}^{n}\left(n_{k+2}\right)_{r-e_{k}}^{n}\left(n_{k+3}\right)_{i-e_{k}}^{n} \\
& \text { - }\left(n_{k}\right)_{i-\varepsilon_{k}}^{n} \overline{\left(n_{k+1}\right)_{i-\varepsilon_{k}}^{n}} \overline{\left(n_{k+2}\right)_{i-\varepsilon_{k}}^{n}}\left(n_{k+3}\right)_{i-\varepsilon_{k}}^{n} \\
& \text { - }\left(n_{k}\right)_{i-e_{k}}^{n} \overline{\left(n_{k+1}\right)_{r-e_{k}}^{n}}\left(n_{k+2}\right)_{i-e_{k}}^{n} \overline{\left(n_{k+3}\right)_{r-e_{k}}^{n}} \\
& \text { - }\left(n_{k}\right)_{r-\tilde{e}_{k}}^{n}\left(n_{k+1}\right)_{r-\tilde{e}_{k}}^{n} \overline{\left(n_{k+2}\right)_{i-e_{k}}^{n}} \overline{\left(n_{k+3}\right)_{r-\varepsilon_{k}}^{n}} \text {. }
\end{aligned}
$$

Now, to prove that the conditions of monotonicity are satisfied on $\mathcal{E}$, we must show for

where

$$
G \equiv\left[G_{k}\right]_{k=0}^{k=3} \geq 0
$$

$$
C_{k} \equiv \frac{\partial}{\partial \mathbf{n}_{i-r_{k}}} H_{k}\left(\mathbf{n}_{i-\tilde{e}_{k}}\right)
$$

that $G_{k} \geq 0$. In this proof, for the sake of brevity, it is understood that unless explicitly stated expressions involving $n_{k}$ for $\hat{k} \in\{k, k+1, k+2, k+3\}$ are all evaluated at $\left(\vec{\imath}-\vec{e}_{k} ; n\right)$. Then for example, $n_{k+1}$ denotes $\left(n_{k+1}\right)_{\vec{r}-\vec{e}_{k}}^{n} ;$ similarly, $\mathbf{n}$ denotes $n_{r-e_{k}}^{n}$. Then with $k$ and $l$ evaluated modulo $d=4$, we have

$$
G_{k, l}=\frac{\partial}{\partial\left(n_{l}\right)_{i-e_{k}}} H_{k}\left(\mathrm{n}_{i-e_{k}}\right)
$$

where

$$
G_{k, k}=\frac{\partial}{\partial\left(n_{k}\right)_{r-e_{k}}} H_{k}\left(\mathbf{n}_{\boldsymbol{r}-\tilde{e}_{k}}\right)
$$




$$
\begin{aligned}
& =n_{k+2} n_{k+3}+n_{k+1} n_{k+2}-n_{k+2} \text {, } \\
& \left(i_{k, k+1}=\frac{i}{i\left(n_{k+1}\right) r-\vec{c}_{k}} I_{k}\left(n_{r-i_{k}}\right)\right. \\
& =-2 n_{k+2} n_{k+3}+n_{k+3}+n_{k} n_{k+2} \text {. } \\
& C_{k, k+2}=\frac{\partial}{\partial\left(n_{k+2}\right)_{r-e_{k}}}-H_{k}\left(n_{i-r_{k}}\right) \\
& =-2 n_{k+1} n_{k+3}+n_{k} n_{k+3}+n n_{k} n_{k+1}-n_{k}+1 \text {, } \\
& C_{k, k+3}=\frac{\partial}{\partial\left(n_{k+3}\right):-e_{k}} H_{k}\left(n_{r-\tilde{e}_{k}}\right) \\
& =-2 n_{k+1} n_{k+2}+n_{k} n_{k+2}+n_{k+1} \text {. }
\end{aligned}
$$

We show that $G_{k} \geq 0$ by showing that $G_{k, 1} \geq 0$.

Note that cach $G_{k, l}$ is a function of three arguments. Let $E \equiv\left[M_{1 .}, M_{+}\right]$. Then the domain of a particular $G_{k, 1}$ is $E^{3}$. Let

$$
\begin{aligned}
& f(\zeta, \rho, \phi)=\rho \phi+\zeta \rho-\rho, \\
& g(\zeta, \rho, \phi)=-2 \rho \phi+\zeta \rho+\phi, \\
& h(\zeta, \rho, \phi)=-2 \rho \phi+\zeta \phi+\zeta \rho-\zeta+1 .
\end{aligned}
$$

Referring to Eqs, 3.23-3.27, we see tliat

$$
\begin{aligned}
& G_{k, k}=f\left(n_{k+1}, n_{k+2}, n_{k+3}\right), \\
& G_{k, k+1}=g\left(n_{k}, n_{k+2}, n_{k+3}\right), \\
& G_{k, k+2}=h\left(n_{k}, n_{k+1}, n_{k+3}\right), \\
& G_{k, k+3}=g\left(n_{k}, n_{k+2}, n_{k+1}\right) .
\end{aligned}
$$

The domain of $f, g$, and $h$ is $E^{3}$. T'o prove $\left(i_{k, l} \geq 0\right.$, it is enough to show that $f(\zeta, \rho, \phi), g(\zeta, \rho, \phi), h(\zeta, \rho, \phi) \geq 0$ for $(\zeta, \rho, \phi) \in t^{3}$. Wo show that the extrene values of $f$ and g are nonnegative. 
Since $f, g$, and $h$ are continuously differentiable in all their arguments, their respective absolute extrema are on the boundary of $E^{3}$, i.e., $\partial E^{3}$, or where the gradients of $f, g$, and $h$ are zero in the interior of $E^{3}$, i.e., $E^{3} \backslash \partial E^{3}$.

Local Extrema of $f, g$, and $h$. We show that $f, g$, and $h$ have no local extrema in the interior of $E^{3}$. We look for solutions to $\nabla_{\zeta, \rho, \phi} f(\zeta, \rho, \phi)=(0,0,0)$, $\nabla_{\zeta, p, \phi} g(\zeta, \rho, \phi)=(0,0,0)$, and $\nabla_{\zeta, \rho, \phi} h(\zeta, \rho, \phi)=(0,0,0)$.

We find

$$
\begin{aligned}
& \nabla_{\zeta, \rho, \phi} f(\zeta, \rho, \phi)=(\rho, \phi+\zeta-1, \rho), \\
& \nabla_{\zeta, \rho, \phi} g(\zeta, \rho, \phi)=(\rho,-2 \phi+\zeta,-2 \rho+\phi), \\
& \nabla_{\zeta, \rho, \phi} h(\zeta, \rho, \phi)=(\phi+\rho-1,-2 \phi+\zeta,-2 \rho+\zeta) .
\end{aligned}
$$

Setting these to $(0,0,0)$, we find no solutions inside of $E^{3}$. Therefore, there are no local extreme.

Boundary Extrema of $f, g$, and $h$. We will show that the boundary extrema of $f, g$, and $h$ are between 0 and 5/9. For each function, $f, g$, and $h$, there are six cases:

$$
\begin{aligned}
& \text { la/b: }(b, \rho, \phi), \quad b \in\left\{M_{-}, M_{+}\right\}, \\
& 2 a / b: \quad(\zeta, \rho, b), \quad b \in\left\{M_{-}, M_{+}\right\}, \\
& 3 a / b: \quad(\zeta, b, \phi), \quad b \in\left\{M_{-}, M_{+}\right\} .
\end{aligned}
$$

Case la/t: Consider $(b, \rho, \phi)$ with $b \in\left\{M_{\ldots}, M_{+}\right\}$and $(\rho, \phi) \in L^{\prime 2}$. Then

$$
f(b, \rho, \phi)=\rho \phi+(b-1) \rho,
$$




$$
\begin{aligned}
& g(b, \rho, \phi)=-2 \rho \phi+b \rho+\phi, \\
& h(b, \rho, \phi)=-2 \rho \phi+b \phi+b \rho-b+1 .
\end{aligned}
$$

We now consider $f, g$, and $h$ as functions of $\rho$ and $\phi$. The local extreme values of these $f, g$, and $h$ are where their gradients (in $\rho$ and $\phi$ ) are zero. The gradients are

$$
\begin{aligned}
& \nabla_{\rho, \phi} f(b, \rho, \phi)=(\phi+b-1, \rho), \\
& \nabla_{\rho, \phi} g(b, \rho, \phi)=(-2 \phi+b,-2 \rho+\phi), \\
& \nabla_{\rho, \phi} h(b, \rho, \phi)=(-2 \phi+b,-2 \rho+b) .
\end{aligned}
$$

For each function, there is no solution for a zero gradient. So, the extrema lie on $\partial E^{2}$. Table 3.2 lists the boundary values.

Case 2a/b: Consider $(\zeta, \rho, b)$ with $b \in\left\{M_{-}, M_{+}\right\}$and $(\zeta, \rho) \in E^{2}$. Then

$$
\begin{aligned}
& f(\zeta, \rho, b)=b \rho+\zeta \rho-\rho \\
& g(\zeta, \rho, b)=-2 b \rho+\zeta \rho+b \\
& h(\zeta, \rho, b)=-2 b \rho+b \zeta+\zeta \rho-\zeta+1 .
\end{aligned}
$$

We now consider $f, g$, and $h$ as functions of $\zeta$ and $\rho$. The local extreme values of these $f, g$, and $h$ are where their gradients (in $\rho$ and $\phi$ ) are zero. The gradients are

$$
\begin{aligned}
& \nabla_{\zeta, \rho} f(\zeta, \rho, b)=(\rho, b+\zeta-1), \\
& \nabla_{\zeta, \rho} g(\zeta, \rho, b)=(\rho,-2 b+\zeta), \\
& \nabla_{\zeta, \rho} h(\zeta, \rho, b)=(b+\rho-1,-2 b+\zeta) .
\end{aligned}
$$


For each function, there is no solution for a zero gradient. So, the extrema lie on $\partial E^{2}$. Table 3.2 lists the boundary values.

Case 3a/b: Consider $(\zeta, b, \phi)$ with $b \in\left\{M_{-}, M_{+}\right\}$and $(\zeta, \phi) \in E^{2}$. Then

$$
\begin{aligned}
& f(\zeta, b, \phi)=b \phi+b \zeta-b, \\
& g(\zeta, b, \phi)=-2 b \phi+b \zeta+\phi, \\
& h(\zeta, b, \phi)=-2 b \phi+b \zeta+\zeta \phi-\zeta+1 .
\end{aligned}
$$

We now consider $f, g$, and $h$ as functions of $\zeta$ and $\phi$. The local extreme values of these $f, g$, and $h$ are where their gradients (in $\rho$ and $\phi$ ) are zero. The gradients are

$$
\begin{aligned}
& \nabla_{\zeta, \phi} f(\zeta, b, \phi)=(b, b), \\
& \nabla_{\zeta, \phi} g(\zeta, b, \phi)=(b,-2 b+1), \\
& \nabla_{\zeta, \phi} h(\zeta, b, \phi)=(b+\phi-1,-2 b+\zeta) .
\end{aligned}
$$

For each function, there is no solution for a zero gradient. So, the extrema lie on $\partial E^{2}$. Table 3.2 lists the boundary values.

Absolute Extrema of $G_{k, l}$. By the preceding analysis, the absolute extrema of $f, g$, and $h$ on $E^{3}$ is given in Table 3.2. We find that

$$
\min G_{k, l}=0 \quad \text { and } \quad \max G_{k, l}=\frac{5}{9} .
$$

Hence, $G_{k, l} \geq 0$ and $H_{k}$ (ergo $H$ ) is a monotonically increasing function in all its arguments. Then lower and upper bounds on $H$ are found at the respective infimum and supremum of all its arguments. Table 3.3 lists the two cases. 
Table 3.2: Boundary Extrema of $f(\zeta, \rho, \phi), g(\zeta, \rho, \phi)$, and $h(\zeta, \rho, \phi)$ in $L_{2}$

\begin{tabular}{||lll|ccc||}
\hline \hline$\zeta$ & $\rho$ & $\phi$ & $f(\zeta, \rho, \phi)$ & $g(\zeta, \rho, \phi)$ & $h(\zeta, \rho, \phi)$ \\
\hline \hline$M_{-}$ & $M_{-}$ & $M_{-}$ & $2 / 9$ & $2 / 9$ & $1 / 3$ \\
$M_{-}$ & $M_{-}$ & $M_{+}$ & $1 / 3$ & $1 / 6$ & $2 / 9$ \\
$M_{-}$ & $M_{+}$ & $M_{-}$ & $5 / 18$ & $1 / 9$ & $2 / 9$ \\
$M_{-}$ & $M_{+}$ & $M_{+}$ & $5 / 12$ & 0 & $1 / 18$ \\
$M_{+}$ & $M_{-}$ & $M_{-}$ & $1 / 3$ & $1 / 3$ & $7 / 18$ \\
$M_{+}$ & $M_{-}$ & $M_{+}$ & $4 / 9$ & $5 / 18$ & $11 / 36$ \\
$M_{+}$ & $M_{+}$ & $M_{-}$ & $5 / 12$ & $1 / 4$ & $11 / 36$ \\
$M_{+}$ & $M_{+}$ & $M_{+}$ & $5 / 9$ & $5 / 36$ & $1 / 6$ \\
\hline \hline
\end{tabular}

Table 3.3: Extrema of $H$ in $\mathrm{LB}_{2}$.

\begin{tabular}{||lllll|llll||}
\hline \hline Extreme Values of Arguments & \multicolumn{4}{|c||}{ Functional Value of $H$} \\
\hline \hline$n_{0}$ & $n_{1}$ & $n_{2}$ & $n_{3}$ & $H_{0}$ & $H_{1}$ & $H_{2}$ & $H_{3}$ \\
\hline$M_{-}$ & $M_{-}$ & $M_{-}$ & $M_{-}$ & $M_{-}$ & $M_{-}$ & $M_{-}$ & $M_{-}$ \\
$M_{+}$ & $M_{+}$ & $M_{+}$ & $M_{+}$ & $M_{+}$ & $M_{+}$ & $M_{+}$ & $M_{+}$ \\
\hline \hline
\end{tabular}


(2) To show $\mathcal{E}$ cannot be extended. Let $\epsilon>0$ be given such that $0 \leq M_{-}-\epsilon<$ $M_{+}+\epsilon \leq 1$. Then let $\mathbf{R}_{-} \equiv\left[M_{-}-\epsilon, M_{+}\right]$and $\mathbf{R}_{+} \equiv\left[M_{-}, M_{+}+\epsilon\right]$, and $\mathbf{M}=\left[M_{-}, M_{+}\right]$. Further, let $\mathbf{E}_{ \pm} \equiv \mathbf{M} \cup \mathbf{E}_{ \pm}$. Then let

$$
\begin{aligned}
& \hat{\mathcal{E}}_{0, \pm} \equiv \mathbf{M} \times \mathbf{M} \times \mathbf{M} \times \mathbf{E}_{ \pm}, \\
& \hat{\mathcal{E}}_{1, \pm} \equiv \mathbf{M} \times \mathbf{M} \times \mathbf{E}_{ \pm} \times \mathbf{M}, \\
& \hat{\mathcal{E}}_{2, \pm} \equiv \mathbf{M} \times \mathbf{E}_{ \pm} \times \mathbf{M} \times \mathbf{M}, \\
& \hat{\mathcal{E}}_{3, \pm} \equiv \mathbf{E}_{ \pm} \times \mathbf{M} \times \mathbf{M} \times \mathbf{M} .
\end{aligned}
$$

It is enough to show that for each $i \in\{0,1,2,3\}$ and $s \in\{+,-\}, G_{k, l}\left(\mathbf{n}^{*}\right)<0$ for some $\mathrm{n}^{*} \in \hat{\mathcal{E}}_{i, s}$. Well, note that from Eq. 3.28 and Table 3.2, we see that (1) $G_{k, k+1}=0$ for $n_{k}=M_{-}$and $n_{k+2}=n_{k+3}=M_{+}$and (2) $G_{k, k+3}=0$ for $n_{k}=M_{-}$and $n_{k+1}=n_{k+2}=M_{+}$. There are eight cases to examine. While we will only present the proof for two cases, the other cases proceed similarly, and the tables we will be using have enough information to verify the other six cases. For each case we use a proof by contradiction.

Case $\hat{\mathcal{E}}_{0,+}=\mathbf{M} \times \mathbf{M} \times \mathbf{M} \times \mathbf{E}_{+}:$Suppose $G_{k, l} \geq 0, \forall k, l \in\{0,1,2,3\}$ and $\forall$ $\mathbf{n} \in \hat{\mathcal{E}}_{0,+}$. Let

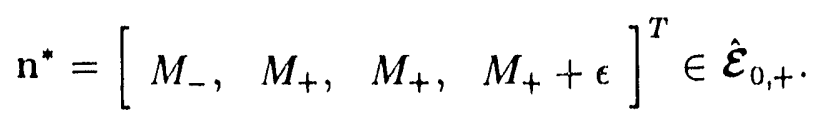

Note that $G_{0,1}^{\prime}=\left(\mathbf{n}^{*}\right)=g\left(M_{-}, M_{+}, M_{+}+\epsilon\right)$. From Table 3.4 we see that $\frac{\partial}{\partial \epsilon} g\left(M_{-}, M_{+}, M_{+}+\epsilon\right)<0$. This coupled with $g\left(M_{-}, M_{+}, M_{+}\right)=0$ (see Table 3.2) implies that $G_{0,1}\left(\mathbf{n}^{*}\right)<0$, which is a contradiction. Therefore, the conditions of monotonicity are not everywhere satisfied in $\hat{\mathcal{E}}_{0,+}$. 
Case $\hat{\mathcal{E}}_{0,-}=\mathbf{M} \times \mathbf{M} \times \mathbf{M} \times \mathbf{E}_{-}:$Suppose $C_{k, l} \geq 0, \quad \forall k, l \in\{0,1,2,3\}$ and $n \in \hat{\mathcal{E}}_{0,-}$. Let

$$
\mathbf{n}^{*}=\left[\begin{array}{lll}
M_{+}, & M_{+}, & M_{+}, M_{-} \epsilon
\end{array}\right]^{T} \in \hat{\mathcal{E}}_{0_{-}-}
$$

Note that $G_{3,0}\left(\mathbf{n}^{*}\right)=g\left(M_{-}-\epsilon, M_{+}, M_{+}\right)$. From Table 3.4 we see that $\frac{\partial}{\partial \epsilon} g\left(M_{-}-\epsilon, M_{+}, M_{+}\right)<0$. This coupled with $g\left(M_{-}, M_{+}, M_{+}\right)=0$ (sec Table 3.2) implies that $G_{3,0}\left(\mathbf{n}^{*}\right)<0$, which is a contradiction. Therefore, the conditions of monotonicity are not everywhere satisfied in $\hat{\mathcal{E}}_{0,-}$.

As noted, the remaining cases proceed similarly, the final result being that none of the eight ways of extending $\mathcal{E}$ produces a region everywhere satisfying the conditions of monotonicity.

We have shown that the conditions of monotonicity are satisfied in $\mathcal{E}$, that no cxtension of $\mathcal{E}$ everywhere satisfies the conditions of monotonicity, and that, $\mathcal{E}$ has volume. Hence, $\mathcal{E}=[2 / 3,5 / 6]^{4}$ is the domain of monotonicity for $\mathrm{LB}_{2}$.

Lemma 3.3 (Discrete Maximum/Minimum Principle for LB $_{2}$ ). Considering the lattice method, $L B_{2}$, let its domain of monotonicity, $\mathcal{E}$, be as in Lemma 3.2. Then $L B_{2}$ has the discrete maximum and minimum principles described in Theorem 2.49

Proof. Note that $\mathbf{M}_{+}=M_{+} \mathbf{q}_{0}$ and $\mathbf{M}_{-}=M_{-} \mathbf{q}_{0}$, and that $\mathcal{C}_{k}\left(\mathrm{M}_{+}\right)=\mathcal{C}_{k}\left(\mathrm{M}_{-}\right)=$ $0 \forall k \in\{0,1,2,3\}$. Therefore, Theorem 2.49 applies, i.e., if $\mathbf{n}_{\mathfrak{i}}^{0} \in \mathcal{E}, \forall \vec{\imath} \in \mathfrak{L}$, then $\mathrm{n}_{\vec{\imath}}^{n} \in \mathcal{E}, \forall \vec{\imath} \in \mathfrak{L}$ for all time steps $n$.

We have established the discrete maximum/minimum principle for $\mathrm{L}_{2} \mathrm{~B}_{2}$. Now, to establish the continuum maximum and minimum principles. 
Table 3.4: Direction of Increase in $f, g$, and $h$ for Arguments Just Outside $\mathcal{E}$ in $\mathrm{LB}_{2}$. The table lists $\frac{\partial}{\partial \epsilon} f, \frac{\partial}{\partial \epsilon} g$, and $\frac{\partial}{\partial \epsilon} h$ for arguments just outside the boundary of $\mathcal{E}$. The arguments are parameterized by $\epsilon>0$.

\begin{tabular}{||lll|lll||}
\hline \hline \multicolumn{2}{||c|}{$\vec{r}(\epsilon)=(\zeta(\epsilon), \rho(\epsilon), \phi(\epsilon))$} & \multicolumn{3}{c||}{} \\
\cline { 1 - 3 }$\zeta(\epsilon)$ & $\rho(\epsilon)$ & $\phi(\epsilon)$ & $\frac{\partial}{\partial \epsilon} f(\vec{r}(\epsilon))$ & $\frac{\partial}{\partial \epsilon} g(\vec{r}(\epsilon))$ & $\frac{\partial}{\partial \epsilon} h(\vec{r}(\epsilon))$ \\
\hline \hline$M_{-}$ & $M_{-}$ & $M_{-}-\epsilon$ & $-2 / 3$ & $+1 / 3$ & $+2 / 3$ \\
$M_{-}$ & $M_{-}$ & $M_{+}+\epsilon$ & $+2 / 3$ & $-1 / 3$ & $-2 / 3$ \\
$M_{-}$ & $M_{+}$ & $M_{-}-\epsilon$ & $-5 / 6$ & $+2 / 3$ & +1 \\
$M_{-}$ & $M_{+}$ & $M_{+}+\epsilon$ & $+5 / 6$ & $-2 / 3$ & -1 \\
$M_{+}$ & $M_{-}$ & $M_{-}-\epsilon$ & $-2 / 3$ & $+1 / 3$ & $+1 / 2$ \\
$M_{+}$ & $M_{-}$ & $M_{+}+\epsilon$ & $+2 / 3$ & $-1 / 3$ & $-1 / 2$ \\
$M_{+}$ & $M_{+}$ & $M_{-}-\epsilon$ & $-5 / 6$ & $+2 / 3$ & $+5 / 6$ \\
$M_{+}$ & $M_{+}$ & $M_{+}+\epsilon$ & $+5 / 6$ & $-2 / 3$ & $-5 / 6$ \\
\cline { 1 - 3 }$M_{-}$ & $M_{-}-\epsilon$ & $M_{-}$ & $-1 / 3$ & $+2 / 3$ & $+2 / 3$ \\
$M_{-}$ & $M_{-}-\epsilon$ & $M_{+}$ & $-1 / 2$ & +1 & +1 \\
$M_{-}$ & $M_{+}+\epsilon$ & $M_{-}$ & $+1 / 3$ & $-2 / 3$ & $-2 / 3$ \\
$M_{-}$ & $M_{+}+\epsilon$ & $M_{+}$ & $+1 / 2$ & -1 & -1 \\
$M_{+}$ & $M_{-}-\epsilon$ & $M_{-}$ & $-1 / 2$ & $+1 / 2$ & $+1 / 2$ \\
$M_{+}$ & $M_{-}-\epsilon$ & $M_{+}$ & $-2 / 3$ & $+5 / 6$ & $+5 / 6$ \\
$M_{+}$ & $M_{+}+\epsilon$ & $M_{-}$ & $+1 / 2$ & $-1 / 2$ & $-1 / 2$ \\
$M_{+}$ & $M_{+}+\epsilon$ & $M_{+}$ & $+2 / 3$ & $-5 / 6$ & $-5 / 6$ \\
\cline { 1 - 2 }$M_{-}-\epsilon$ & $M_{-}$ & $M_{-}$ & $-2 / 3$ & $-2 / 3$ & $-1 / 3$ \\
$M_{-}-\epsilon$ & $M_{-}$ & $M_{+}$ & $-2 / 3$ & $-2 / 3$ & $-1 / 2$ \\
$M_{-}-\epsilon$ & $M_{+}$ & $M_{-}$ & $-5 / 6$ & $-5 / 6$ & $-1 / 2$ \\
$M_{-}-\epsilon$ & $M_{+}$ & $M_{+}$ & $-5 / 6$ & $-5 / 6$ & $-2 / 3$ \\
$M_{+}+\epsilon$ & $M_{-}$ & $M_{-}$ & $+2 / 3$ & $+2 / 3$ & $+1 / 3$ \\
$M_{+}+\epsilon$ & $M_{-}$ & $M_{+}$ & $+2 / 3$ & $+2 / 3$ & $+1 / 2$ \\
$M_{+}+\epsilon$ & $M_{+}$ & $M_{-}$ & $+5 / 6$ & $+5 / 6$ & $+1 / 2$ \\
$M_{+}+\epsilon$ & $M_{+}$ & $M_{+}$ & $+5 / 6$ & $+5 / 6$ & $+2 / 3$ \\
\hline \hline
\end{tabular}


Lemma 3.4 (Hydrodynamical Maximum/Minimum Principle ron LB $_{2}$ ). Lemma 2.55 holds with $D(u)=\frac{1}{2(1-u)(1+u)}-\frac{1}{2}$.

Proof. The arguments follow those for Lemma 2.55, except that the smooth extension of $D(u)$ is near $u=-1$ and $u=+1$ instead of $u=0$ and $u=1$.

Lemma 3.5 (Continuum Maximum/Minimum Principle for L $B_{2}$ ). Lemma Q.56 holds with $\mathrm{h}^{(0)}, \mathrm{h}^{(1)}, \mathrm{h}^{(2)}$, and $\mathrm{h}^{(3)}$ defined in Eqs. 3.19.3.22, and $u \in C^{4}\left(\mathcal{R}^{2}, \mathcal{R}\right)$ and $\sigma^{(2)} \in C^{2}\left(\mathcal{R}^{2}, \mathcal{R}\right)$ solutions of Eq. 3.6 and Eq. 3.1., respectively.

Proof. The arguments follow those for Lemma 2.56 .

Corollary 3.6. In $L B_{2}, \exists \delta_{0}>0$ such that $\forall \delta \in\left(0, \delta_{0}\right)$, if $h_{\vec{\imath}}^{0} \in \mathcal{E}, \forall \vec{\imath} \in \mathfrak{L}$, then $\mathrm{h}_{\overrightarrow{\mathfrak{t}}}^{n} \in \mathcal{E}, \forall \vec{\imath} \in \mathfrak{L} \forall n$, where $\mathcal{E}$ is the domain of monotonicity for $L B_{\mathscr{Z}}$.

Proof. Let $R_{+} \equiv M_{+}<1$ and $R_{-} \equiv M_{-}>0$, where $M_{+}$and $M_{-}$are defined in Lemmi 3.2. Then apply Lemma 3.5 to yield the result.

We have established continuum maximum and minimum principles for the truncated equilibrium expansion, $\mathbf{h}$. The sufficient conditions are that (1) $\sigma^{(0)}$ has a maximum/minimum principle, (2) finite global bounds on $\mathbf{h}^{(1)}, \mathbf{h}^{(2)}$, and $\mathbf{h}^{(3)}$ exist $^{5}$, and (3) expansion parameter $\delta>0$ is sufficiently small.

LEMMA 3.7 (Stability of $\mathrm{LB}_{2}$ ). Let $\mathbf{n}^{n}, \mathrm{~h}^{n}$ and $L\left[\mathrm{n}^{n}, \mathrm{~h}^{n}\right]$ be defined as in Definition 2.41. Let domain of monotonicity, $\mathcal{E}$, be as in Lemma 3.2. Suppose $\mathrm{n}_{\tilde{i}}^{0},\left(\mathrm{~h}^{(0)}\right)_{i}^{0} \in \mathcal{E} .^{6}$ And suppose the hypotheses of Lemma 3.5 are satisfied. Then $\exists$ $\tau>0$ such that $\left\|L\left[\mathbf{n}^{n}, \mathbf{h}^{n}\right]\right\|_{\ell_{1}} \leq 1 \forall n$ such that $0 \leq n \Delta t \leq T$ and $0<\Delta t<\tau$.

Proof. Let $R_{+}=M_{+}$and $R_{-}=M_{-}$, where $M_{+}$and $M_{-}$are defined in Lemma

\footnotetext{
5These are guarenteed to exist by regularity of the $\mathcal{O}\left[\delta^{2}\right]$ and $\mathcal{O}\left[\delta^{4}\right]$ consistency conditions, Egs. 3.6 and 3.14 , respectively.

${ }^{6}$ Recall that $\mathbf{h}^{(0)}$ is the equilibrium in the truncated equilibrium expansion, $\mathbf{h}=\sum_{j=0}^{3} \delta^{j} \mathbf{h}^{(j)}\left(S^{(S e}\right.$
} E(1. 3.17). 
3.2. Let $\delta_{0}$ be given by Lemma 3.5. Choose $\tau=T\left(\delta_{0}\right)^{2}$ so that $\Delta x \leq L \delta_{0}$. Note that Corollary 3.6 applies to yield that $\mathrm{h}^{n} \in \mathcal{E}, \forall n$ such that $0 \leq n \Delta t \leq T$ and $0<\Delta t<\tau$. Then $\left\|L\left[\mathbf{n}^{n}, \mathbf{h}^{n}\right]\right\|_{\ell_{1}} \leq 1$ by Lemma 2.59 .

Theorem 3.8 (Convergence of $\mathrm{LB}_{2}$ ). Let the conditions of Lemmas 3.1, 3.3, and 3.5 be satisfied. Then $\lim _{\Delta t \rightarrow 0} \frac{1}{\Delta t}\left\|F^{n}\right\|_{\ell_{1}}=0, \forall n \ni 0 \leq n \Delta t \leq T$, in which $\Delta t=(\Delta x)^{2} /(2 \nu)$, and $\Delta x=L \delta, \Delta t=T \delta^{2}$, and $\nu=L^{2} /(2 T)$ for spatial and temporal scale lengths $L$ and $T$.

Proof. This is a consequence of Theorem 2.61 .

We can recover an $\mathcal{O}\left[\delta^{2}\right]$ approximation to $u(x, y ; t)$ by the covergence arguments, the definition of the truncated equilibrium expansion, and consistency arguments to obtain that hydrodynamical error,

$$
\begin{aligned}
\left(f_{0}\right)_{\mathfrak{i}}^{n} & =\frac{1}{4} \mathbf{q}_{0} \cdot \mathbf{e}_{\mathfrak{i}}^{n}+\mathcal{O}\left[\delta^{2}\right] \\
& =\mathbf{p}_{\mathfrak{i}}^{n}-\rho_{\mathfrak{i}}^{n}+\mathcal{O}\left[\delta^{2}\right] \\
& =\frac{1}{4} \sum_{k=0}^{3}\left(n_{k}\right)_{\mathfrak{i}}^{n}-\frac{1}{4} \sum_{k=0}^{3}\left(h_{k}\right)_{\mathfrak{i}}^{n}+\mathcal{O}\left[\delta^{2}\right] \\
& =\frac{1}{4} \sum_{k=0}^{3}\left(n_{k}\right)_{\mathfrak{i}}^{n}-\frac{1}{4}\left(\sum_{k=0}^{3}\left(h_{k}^{(0)}\right)_{\mathfrak{i}}^{n}+\mathcal{O}\left[\delta^{2}\right]\right)+\mathcal{O}\left[\delta^{2}\right] \\
& =\frac{1}{4} \sum_{k=0}^{3}\left(n_{k}\right)_{\mathfrak{i}}^{n}-u_{\mathfrak{i}}^{n}+\mathcal{O}\left[\delta^{2}\right] \\
& =\frac{1}{4} \sum_{k=0}^{3}\left(n_{k}\right)_{\mathfrak{i}}^{n}-u(\vec{\imath} \Delta x ; n \Delta t)+\mathcal{O}\left[\delta^{2}\right] .
\end{aligned}
$$

We have shown that the hydrodynamical mode of the lattice Boltzmann method, $\mathrm{LB}_{2}$, converges $\mathcal{O}\left[\delta^{2}\right]$ to the solution of its hydrodynamical equation, Eq. 3.6. Assumptions and statements used to obtain convergence include: (1) monotonicity principles of the lattice method, which restricts the domain of the occupation numbers, (2) boundedness of the solutions, $u$ and $\sigma^{(2)}$ (and up to their fourth spatial derivatives), to the 
$\mathcal{O}\left[\delta^{2}\right]$ and $\mathcal{O}\left[\delta^{4}\right]$ consistency conditions, and (3) a (continumm) maximum/minim!mu minciple on $u$.

\subsection{A Lattice Method for the 1-D Viscous Burg- ers Equation}

This section presents numerical analysis of a lattice method for the one-dimensional viscous Burgers equation,

$$
\rho_{t}+\rho \rho_{x}=\nu \rho_{x x}
$$

Boghosian and Levermore [7] introduced a lattice gas method for this equation. Here, we present analysis of this method as a lattice Boltzmann method under the framework set up in Chapter 2. The main purpose of this example is to point out how a collision operator with an $\mathcal{O}[\delta]$ component affects the analysis. It also fills in some of the details of the analysis in [7]. Considering the results of [53], regarding correlations for the lattice gas method, our lattice Boltzmann analysis also extends to the lattice gas case. (Lebowitz, et al. [53] showed that the correlations may safely be neglected, i.e., that particles are statistically uncorrelated prior to collisions.) While presenting computational results for the lattice Boltzmann method, we also use the corresponding lattice gas method in a forthcoming chapter on domain decomposition for lattice methods. We find the lattice Boltzmann method to be a second-order convergent. finite difference method. 


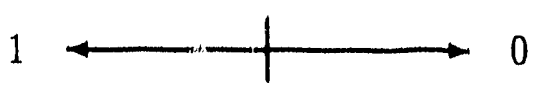

Figure 3.2: Direction labels for a lattice method for Eq. 3.29.

\subsubsection{Collision Rules}

There are two directions, labeled 0 and 1. Their orientation is depicted in Figure 3.2, with 0 to the right and 1 left. Table 3.5 and Figure 3.3 exhibit the collision rules. The probability of an advection in direction 0 (to the right) is $a$; in opposite direction it is $\bar{a}=1-a$. We assume that

$$
a=\frac{1+\epsilon}{2} \text { and } \bar{a}=\frac{1-\epsilon}{2}
$$

where for some constant $K>0, \epsilon=K \delta$ is the advection bias (to the right). ${ }^{7}$ The assumption that $\epsilon=\mathcal{O}[\delta]$ is crucial in recovering Eq. 3.29 from the dynamics of the method.

\subsubsection{Collision Operator}

By examining the collision rules (Table 3.5), we find the collision operator to be given by

$$
\begin{aligned}
& \dot{C}_{0}(\hat{\mathbf{n}})=\underbrace{+a \overline{n_{0}} \hat{n}_{1}}_{\text {Rule } 1 \mathrm{~b}} \underbrace{-\bar{a} \hat{n}_{0} \overline{\hat{n}_{1}}}_{\text {Rule } 2 \mathrm{a}} \\
& \mathcal{C}_{1}(\hat{\mathbf{n}})=\underbrace{-a \hat{n}_{0} \hat{n}_{1}}_{\text {Rule } 1 \mathrm{~b}} \underbrace{+\bar{a} \hat{n}_{0} \overline{\hat{n}_{1}}}_{\text {Rule } 2 \mathrm{a}},
\end{aligned}
$$

\footnotetext{
${ }^{7}$ The advection bias is denoted $\epsilon$ in the present text while it is denoted $\alpha$ in [7].
} 


\section{PRE-COLLISION POST-COLLISION PROBABBILITY}

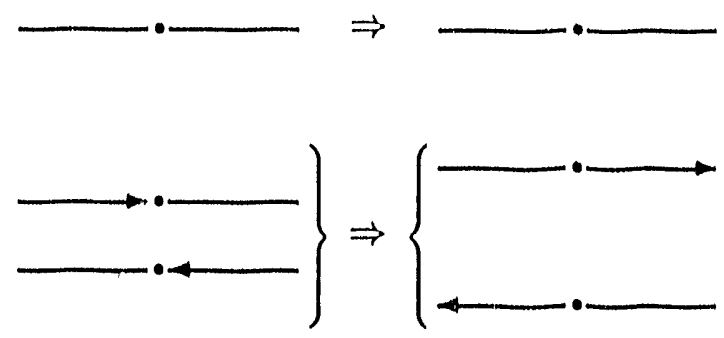

1

$$
\begin{aligned}
& a=\frac{1+\epsilon}{2} \\
& \bar{a}=\frac{1-\epsilon}{2}
\end{aligned}
$$

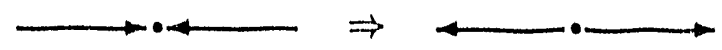

Figure 3.3: Collision rules of a lattice method for the one-dimensional viscous Burg. ers equation.

\begin{tabular}{|c|c|c|c|c|c|}
\hline \multirow[t]{2}{*}{$\overline{\text { Rule }}$} & \multicolumn{2}{|c|}{$\bar{\alpha}$} & \multicolumn{2}{|c|}{$\bar{\beta}$} & \multirow[t]{2}{*}{$\overline{\mathcal{S}(\alpha \rightarrow \beta)}$} \\
\hline & $\alpha_{0}$ & $\alpha_{1}$ & $\beta_{0}$ & $\overline{\beta_{1}}$ & \\
\hline$\overline{0}$ & $\overline{\overline{0}}$ & $\overline{0}$ & 0 & $\overline{0}$ & $\overline{\overline{1}}$ \\
\hline $1 \mathrm{a}$ & 0 & 1 & 0 & 1 & $\bar{a}$ \\
\hline $1 b$ & 0 & 1 & 1 & 0 & $a$ \\
\hline $2 a$ & 1 & $\overline{0}$ & 0 & 1 & $\bar{a}$ \\
\hline $2 b$ & 1 & 0 & 1 & 0 & $a$ \\
\hline 3 & 1 & 1 & & 1 & 1 \\
\hline
\end{tabular}

Table 3.5: Collision Rules of a Lattice Method for Eq. 3.29. 
or, in vector notation,

$$
\mathcal{C}(\hat{\mathbf{n}})=\left[\begin{array}{c}
+a \hat{n}_{0} \hat{n}_{1}-\pi \hat{n}_{0} \bar{n}_{1} \\
-a \hat{n}_{0} \hat{n}_{1}+a \hat{n}_{0} \bar{n}_{1}
\end{array}\right]=\left(\frac{1}{2}\left(-\hat{n}_{0}+\hat{n}_{1}\right)+\frac{\epsilon}{2}\left(\hat{n}_{0}+\hat{n}_{1}-2 \hat{n}_{0} \hat{n}_{1}\right)\right)\left[\begin{array}{c}
+1 \\
-1
\end{array}\right] .
$$

$\hat{n}_{k}$ is the occupation number for direction $k$, i.e., the number of particles moving in direction $k$.

\subsubsection{Lattice Boltzmann Approximation}

The exact ensembled collision operator is given by

$$
\begin{aligned}
\langle\mathcal{C}(\hat{\mathbf{n}})\rangle & =\mathcal{C}(\mathbf{n})+\mathbf{C} \\
& =\left[\begin{array}{l}
+a \pi_{0} n_{1}-\pi n_{0} \pi_{1} \\
-a \pi_{0} n_{1}+\pi n_{0} \pi_{1}
\end{array}\right]+\left[\begin{array}{l}
+a \operatorname{Cov}\left(\overline{\hat{n}_{0}}, \hat{n}_{1}\right)-\pi \operatorname{Cov}\left(\hat{n}_{0}, \overline{\hat{n}_{1}}\right) \\
-a \operatorname{Cov}\left(\overline{\hat{n}_{0}}, \hat{n}_{1}\right)+\overline{\operatorname{Cov}}\left(\hat{n}_{0}, \overline{\hat{n}_{1}}\right)
\end{array}\right] \\
& =\left(\frac{1}{2}\left(-n_{0}+n_{1}\right)+\frac{\epsilon}{2}\left(n_{0}+n_{1}-2 n_{0} n_{1}\right)-\epsilon \operatorname{Cov}\left(\hat{n}_{0}, \hat{n}_{1}\right)\right)\left[\begin{array}{l}
+1 \\
-1
\end{array}\right] \\
& =\mathcal{C}^{(0)}(\mathbf{n})+\delta \mathcal{C}^{(1)}(\mathbf{n})+\mathbf{C},
\end{aligned}
$$

where $\mathcal{C}^{(0)}(\mathbf{n})$ is the $\mathcal{O}[1]$ coefficient, $\mathcal{C}^{(1)}(\mathbf{n})$ is the $\mathcal{O}[\delta]$ coefficient, and $\mathbf{C}$ involves the covariances. 'To get the third line of the above, a certain relationship for covariances is used. Let $\hat{p}$ and $\hat{q}$ be instances of some infinite collection of events or samples. Then

$$
\begin{aligned}
\operatorname{Cov}(\overline{\hat{p}}, \hat{q}) & =\langle\overline{\hat{p}} \hat{q}\rangle-\langle\hat{\hat{p}}\rangle\langle\hat{q}\rangle \\
& =\langle\hat{q}-\hat{p} \hat{q}\rangle-\langle 1-\hat{p}\rangle\langle\hat{q}\rangle \\
& =\langle\hat{q}\rangle-\langle\hat{p} \hat{q}\rangle-\langle\hat{q}\rangle+\langle\hat{p}\rangle\langle\hat{q}\rangle \\
& =-(\langle\hat{p} \hat{q}\rangle-\langle\hat{p}\rangle\langle\hat{q}\rangle) \\
& =-\operatorname{Cov}(\hat{p}, \hat{q}) .
\end{aligned}
$$

\footnotetext{
"Because we keep track of the covariances, this equation is exact; it is not an approximation.
} 


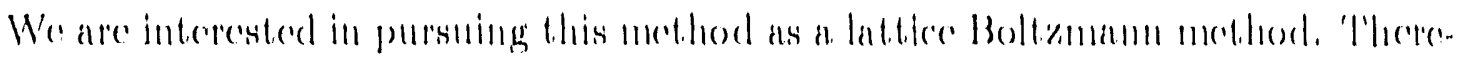

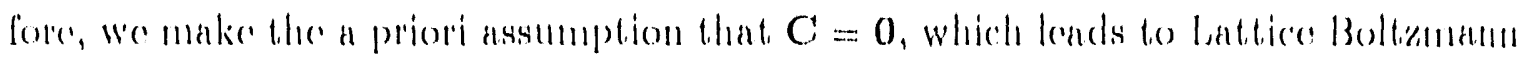
licpllation,

$$
\mathcal{A} \mathbf{n}=\mathbf{n}+\mathcal{C}(\mathbf{n})
$$

where

$$
\mathcal{C}(\mathrm{n})=\mathcal{C}^{(())}(\mathrm{n})+\delta \mathrm{C}^{(1)}(\mathrm{n})
$$

and

$$
\begin{aligned}
& \mathcal{C}^{(1)}(\mathrm{n})=\frac{1}{2}\left(-n_{0}+n_{1}\right)\left[\begin{array}{l}
+1 \\
-1
\end{array}\right] \\
& \mathcal{C}^{(1)}(\mathrm{n})=\frac{\kappa}{2}\left(n_{0}+n_{1}-2 n_{0} n_{1}\right)\left[\begin{array}{c}
+1 \\
-1
\end{array}\right]
\end{aligned}
$$

and the advection operator is defined by

$$
\mathcal{A n}_{i}^{n}=\left[\left(u_{k}\right)_{i+\vec{e}_{k}}^{n+1}\right]_{k=0}^{1}
$$

for unit velocity vectors, $\vec{e}_{0}=+1$ and $\vec{e}_{1}=-1$. Then $\mathbf{n}$ is the vector of mean occupation numbers.

\subsubsection{Equilibrium Analysis}

It is convenient to use the direct mothod of Section 2.2 .1 for detcrminimg the equilit). rimm, Setting the leading term of the collision operater to zoro, we get

$$
\mathcal{C}^{(0)}(\mathbf{n})=\left[\begin{array}{l}
0 \\
0
\end{array}\right] \Rightarrow \frac{1}{2}\left(-n_{0}+n_{1}\right)=0 \Rightarrow n_{0}=n_{1}=u
$$


Then the equilibrium mat be written

$$
\mathbf{n}^{(0)} \equiv\left[\begin{array}{l}
u \\
u
\end{array}\right]
$$

\subsubsection{Linearized Collision Operator}

Using the non-trivial equilibrium solution, $\mathbf{n}^{(0)}$, wo can now linearize the collision operator. But first, recall that only the leading order term of the collision operator is used in the linearization (sce Definition 2.32). 'Then the linearized collision operator' is given by

$$
\mathcal{L}=\left.\frac{\partial}{\partial \mathbf{n}} \mathcal{C}^{(0)}(\mathbf{n})\right|_{\mathbf{n}=\mathbf{n}^{(0)}}=\left.\left[\frac{\partial}{\partial m_{1}} \mathcal{C}_{k}^{(0)}(\mathbf{n})\right]_{k, l=0}^{1}\right|_{\mathbf{n}=\mathbf{n}^{(0)}} .
$$

There are four cases:

$$
\begin{aligned}
& \left.\frac{\partial}{\partial n_{0}} \mathcal{C}_{0}^{(0)}(\mathbf{n})\right|_{\mathbf{n}=\mathrm{n}^{(0)}}=-\frac{1}{2},\left.\quad \frac{\partial}{\partial n_{1}} \mathcal{C}_{0}^{(\mathrm{u})}(\mathbf{n})\right|_{\mathbf{n}=\mathrm{n}^{(0)}}=+\frac{1}{2} \\
& \left.\frac{\partial}{\partial n_{0}} \mathcal{C}_{1}^{(0)}(n)\right|_{n=n^{(0)}}=+\frac{1}{2},\left.\quad \frac{\partial}{\partial n_{1}} \mathcal{C}_{1}^{(0)}(n)\right|_{n=n^{(0)}}=-\frac{1}{2} .
\end{aligned}
$$

Then the linearized collision operator, $\mathcal{L}$, written in matrix notution is

$$
\mathcal{L}=\frac{1}{2}\left[\begin{array}{ll}
-1 & +1 \\
+1 & -1
\end{array}\right]
$$

Clearly, $\mathcal{L}$ is a symmetric, nonpositive definite circulant.

\subsubsection{Eigenvalues and Eigenvectors}

The eigenvalues of $\mathcal{L}$ are given by

$$
\text { eigenvalues }(\mathcal{L})==\left(\lambda_{0}, \lambda_{1}\right)=(0,-\cdots) \text {. }
$$


Table 3.6: ('omponentwise Eigenvertor Products.

\begin{tabular}{||l||l|l||}
\hline$\star \star$ & $q_{0}$ & $q_{1}$ \\
\hline$q_{0}$ & $q_{0}$ & $q_{1}$ \\
\hline$q_{1}$ & $q_{1}$ & $q_{0}$ \\
\hline
\end{tabular}

The unnormalized "ienvectors of $\mathcal{L}$ are given by its eigenmatrix,

$$
\begin{aligned}
\mathbf{Q} & =\left[\begin{array}{ll}
\mathbf{q}_{0}, & \mathbf{q}_{1}
\end{array}\right] \\
& =\left[\begin{array}{ll}
+1 & +1 \\
+1 & -1
\end{array}\right] .
\end{aligned}
$$

The nullspace of the linearized collision operator is spanned by the eigenvectors corresnonding to zero eigenvalues, i.e., nullspace $(\mathcal{L})=\operatorname{span}\left(\mathbf{q}_{0}\right)$. The pseudo-inverse of $\mathcal{L}$ can be written

$$
\mathcal{L}^{+}=-\frac{\mathbf{q}_{1} \mathbf{q}_{1}^{T}}{\mathbf{q}_{1} \cdot \mathbf{q}_{1}}=-\frac{1}{2} \mathbf{q}_{1} \mathbf{q}_{1}^{T}=-\frac{1}{2}\left[\begin{array}{ll}
+1 & -1 \\
-1 & +1
\end{array}\right]
$$

The componentwise eigenvector products ( $\star$ operation) are summarized in Table 3.6.

\subsubsection{Discrete Chapman-Enskog Expansion}

To apply the discrete Chapman-Enskog procedure of Section 2.3, we "sume

$$
\begin{aligned}
\epsilon & =K \delta \\
\Delta x & =L \delta \\
\Delta t & =T \delta^{2}
\end{aligned}
$$

\footnotetext{
${ }^{9}{ }_{10}$ and $q_{1}$ are column vectors.
} 
in which $K>0$, and $L>0$ and $T>0$ are the respective spatial and temporal scale lengths.

Note that gradient expressions involving the unit velocity vectors may be generally expressed as follows:

$$
\left[\left(\vec{e}_{k} \cdot \nabla\right)^{j}\right]_{k=0}^{1}= \begin{cases}\frac{\partial^{j}}{\partial x^{j}} \mathbf{q}_{0}, & j \text { even, } \\ \frac{\partial^{j}}{\partial x^{j}} \mathbf{q}_{1}, & j \text { odd. }\end{cases}
$$

Now, we may readily apply the discrete Chapman-Enskog procedure. We will use the following notation:

$$
\begin{aligned}
A(u) & \equiv u(1-u) \\
A^{\prime}(u) & =1-2 u \\
\mathbf{g}^{(j)} & =\mathcal{L} \cdot \mathbf{n}^{(j)}-\sum_{k=0}^{1} \tilde{c}^{(j)} \mathbf{q}_{k} \\
\mathbf{n}^{(j)} & =\sigma^{(j)} \mathbf{q}_{0}+\mathcal{L}^{+} \cdot \mathbf{g}^{(j)}=\sum_{k=0}^{1} c^{(j)} \mathbf{q}_{k}
\end{aligned}
$$

in which $c_{0}^{(j)}=\sigma^{(j)}$ and $c_{1}^{(j)}=-\tilde{c}_{1}^{(j)}{ }^{10}$ We will be using a third order truncated equilibrium expansion, i.e., $\mathbf{h}=\sum_{j=0}^{3} \delta^{j} \mathbf{h}^{(j)}$, in which $\mathbf{h}^{(j)}=\mathbf{n}^{(j)}$. And as $\sigma^{(3)}$ appears in $\mathbf{n}^{(3)}$, and $\sigma^{(3)}$ is determined by the $\mathcal{O}\left[\delta^{5}\right]$ consistency condition, we carry out the discrete Chaprnan-Enskog procedure to determine the consistency condition at $\mathcal{O}\left[\delta^{5}\right]$. For each order in matching the advection and collision operator expansions, Eq. 2.24 and Eq. 2.18, respectively, we find the following:

$\mathcal{O}[1]: \mathcal{C}^{(0)}\left(\mathbf{n}^{(0)}\right)=0$ as desired, si $\mathrm{e}$

$$
\mathbf{n}^{(0)}=\left[\begin{array}{l}
u \\
u
\end{array}\right]
$$

$$
{ }^{10} c_{1}^{(j)}=\frac{1}{\lambda_{1}} \tilde{c}_{1}^{(j)} \text { and } \lambda_{1}=-1
$$


is an equilibrium.

$\mathcal{O}[\delta]:$ We find

$$
\begin{aligned}
\mathcal{L} \cdot \mathbf{n}^{(1)} & =L\left[\vec{e}_{k} \cdot \nabla\right] \mathbf{n}^{(0)}-\mathcal{C}^{(1)}\left(\mathbf{n}^{(0)}\right) \\
& \equiv \mathbf{g}^{(1)} \\
& =\sum_{k=0}^{1} \tilde{c}_{k}^{(1)} \mathbf{G}_{k},
\end{aligned}
$$

where

$$
\begin{aligned}
& \tilde{c}_{0}^{(1)}=0, \\
& \tilde{c}_{0}^{(1)}=L u_{x}-K A(u) .
\end{aligned}
$$

- Consistency: Note that already $\mathbf{g}^{(1)}$ is orthogonal to the nullspace of $\mathcal{L}$, i.e., $\mathrm{q}_{0} \cdot \mathrm{g}^{(1)}=0$, so that no consistency condition is introduced at this order.

- Solve for $n^{(1)}$ : We have

$$
\begin{aligned}
\mathbf{n}^{(1)} & =\mathcal{L}^{+} \cdot \mathbf{g}^{(1)}+\sigma^{(1)} \mathbf{q}_{0} \\
& =\sum_{k=0}^{1} c_{k}^{(1)} \mathbf{q}_{k},
\end{aligned}
$$

where

$$
\begin{aligned}
& c_{0}^{(1)}=\sigma^{(1)}, \\
& c_{1}^{(1)}=K A(u)-L u_{x},
\end{aligned}
$$

and $\sigma^{(1)}$ is the arbitrary parameter introduced by the nullspace of the linearized collision operator. ${ }^{11}$

\footnotetext{
${ }^{11}$ One parameter is introduced since $\operatorname{dim}($ nullspace $(\mathcal{L}))=1$.
} 
$\mathcal{O}\left[\delta^{2}\right]:$ We find

$$
\begin{aligned}
\mathcal{L} \cdot \mathbf{n}^{(2)} & =\frac{L^{2}}{2}\left[\left(\vec{e}_{k} \cdot \nabla\right)^{2}\right] \mathbf{n}^{(0)}+T \frac{\partial}{\partial t} \mathbf{r}^{(0)}+L\left[\vec{e}_{k} \cdot \nabla\right] \mathbf{n}^{(1)} \\
& -\frac{1}{2} \mathcal{D}^{2} \mathcal{C}^{(0)}\left(\mathbf{n}^{(0)}\right) \cdot \mathbf{n}^{(1)} \mathbf{n}^{(1)}-\mathcal{D} \mathcal{C}^{(1)}\left(\mathbf{n}^{(0)}\right) \cdot \mathbf{n}^{(1)} \\
& \equiv \mathbf{g}^{(2)} \\
& =\sum_{k=0}^{1} \tilde{c}_{k}^{(2)} \mathrm{q}_{k}
\end{aligned}
$$

where

$$
\begin{aligned}
& \tilde{c}_{0}^{(2)}=T u_{t}+K L \frac{\partial}{\partial x} A(u)-\frac{L^{2}}{2} u_{x x}, \\
& \tilde{c}_{1}^{(2)}=L \sigma_{x}^{(1)}-K A^{\prime}(u) \sigma^{(1)},
\end{aligned}
$$

and in which

$$
\begin{aligned}
& \mathcal{D}^{2} \mathcal{C}^{(0)}\left(\mathbf{n}^{(0)}\right) \cdot \mathrm{n}^{(1)} \mathrm{n}^{(1)}=0, \\
& \mathcal{D C} \mathcal{C}^{(1)}\left(\mathrm{n}^{(0)}\right) \cdot \mathrm{n}^{(1)} \quad=K A^{\prime \prime}(u) \sigma^{(1)} \mathrm{q}_{1} \text {. }
\end{aligned}
$$

- Consistency: Setting $\mathbf{q}_{0} \cdot \mathbf{g}^{(2)}=0$ yields the $\mathcal{O}\left[\delta^{2}\right]$ consistency condition,

$$
\frac{\partial}{\partial t} u+c \frac{\partial}{\partial x} A(u)=\nu \frac{\partial^{2}}{\partial x^{2}} u
$$

where

$$
\begin{aligned}
& c=\frac{K L}{T}, \\
& \nu=\frac{L^{2}}{2 T} .
\end{aligned}
$$

With the change of variable, ${ }^{12} \rho=c A^{\prime}(u)$, a linear transformation, we find that $\rho$ satisfies Eq. 3.29. (This is identical to the result of [7]. ${ }^{13}$ ) The transformation

\footnotetext{
${ }^{12}$ Recall from the beginning of this subsection, i.e., Section 3.2 .7 , that $A(u)=u(1-u)$ and $A^{\prime}(u)=1-2 u$.

${ }^{13}$ Our $\rho$ is their $u$.
} 
is consistent with the following parameters:

$$
\begin{aligned}
\Delta x & \equiv L \delta \\
\Delta t & \equiv T \delta^{2}=\frac{(\Delta x)^{2}}{2 \nu}, \\
\epsilon & \equiv K \delta=\frac{c \Delta x}{2 \nu} \\
c & \neq 0 .
\end{aligned}
$$

- Solve for $\mathrm{n}^{(2)}$ : We have

$$
\begin{aligned}
\mathbf{n}^{(2)} & =\mathcal{L}^{+} \cdot \mathbf{g}^{(2)}+\sigma^{(2)} \mathbf{q}_{0} \\
& =\sum_{k=0}^{1} c_{k}^{(2)} \mathbf{q}_{k},
\end{aligned}
$$

where

$$
\begin{aligned}
& c_{0}^{(2)}=\sigma^{(2)}, \\
& c_{1}^{(2)}=K A^{\prime}(u) \sigma^{(1)}-L \sigma_{x}^{(1)},
\end{aligned}
$$

in which $\sigma^{(2)}$ is the arbitrary parameter (a scalar) introduced by the nullspace of the linearized collision operator.

$\mathcal{O}\left[\delta^{3}\right]:$ We find

$$
\begin{aligned}
\mathcal{L} & \cdot \mathbf{n}^{(3)} \\
& =\left[\frac{L^{3}}{3 !}\left(\vec{e}_{k} \cdot \nabla\right)^{3}+L T\left(\vec{e}_{k} \cdot \nabla\right) \frac{\partial}{\partial t}\right] \mathbf{n}^{(0)}+\left[\frac{L^{2}}{2 !}\left(\vec{e}_{k} \cdot \nabla\right)^{2}+T \frac{\partial}{\partial t}\right] \mathbf{n}^{(1)} \\
& +\left[L\left(\vec{e}_{k} \cdot \nabla\right)\right] \mathbf{n}^{(2)}-\frac{1}{6} \mathcal{D}^{3} \mathcal{C}^{(0)}\left(\mathbf{n}^{(0)}\right) \cdot \mathbf{n}^{(1)} \mathbf{n}^{(1)} \mathbf{n}^{(1)}-\mathcal{D}^{2} \mathcal{C}^{(0)}\left(\mathbf{n}^{(0)}\right) \cdot \mathbf{n}^{(1)} \mathbf{n}^{(2)} \\
& -\frac{1}{2} \mathcal{D}^{2} \mathcal{C}^{(1)}\left(\mathbf{n}^{(0)}\right) \cdot \mathbf{n}^{(1)} \mathbf{n}^{(1)}-6 \mathcal{D} \mathcal{C}^{(1)}\left(\mathbf{n}^{(0)}\right) \cdot \mathbf{n}^{(2)} \\
& \equiv \mathbf{g}^{(3)}
\end{aligned}
$$


where

$$
\begin{aligned}
\tilde{c}_{0}^{(3)}= & K L \frac{\partial}{\partial x}\left(A^{\prime}(u) \sigma(\zeta)-\frac{L^{2}}{2} \sigma_{x x}^{(1)}+T \sigma_{t}^{(1)}\right. \\
\tilde{c}_{1}^{(3)=} & -\frac{L^{3}}{3} u_{x x x}+K\left(\frac{L^{2}}{\partial} \frac{\partial}{\partial x}+T \frac{\partial}{\partial t}\right) A^{\prime}(u)+L \sigma_{x}^{(2)}-6 K A^{\prime}(u) \sigma^{(2)} \\
& +K\left[\left(\sigma^{(2)}\right)^{2}-\left(L u_{x}-K A(u)\right)^{2}\right]
\end{aligned}
$$

and in which

$$
\begin{array}{ll}
\mathcal{D}^{3} \mathcal{C}^{(0)}\left(\mathrm{n}^{(0)}\right) \cdot \mathrm{n}^{(1)} \mathrm{n}^{(1)} \mathrm{n}^{(1)} & =0, \\
\mathcal{D}^{2} \mathcal{C}^{(0)}\left(\mathrm{n}^{(0)}\right) \cdot \mathrm{n}^{(1)} \mathrm{n}^{(2)} & =0, \\
\mathcal{D}^{2} \mathcal{C}^{(1)}\left(\mathrm{n}^{(0)}\right) \cdot \mathrm{n}^{(1)} \mathrm{n}^{(1)} & =2 K\left[\left(L u_{x}-K A(u)\right)^{2}-\left(\sigma^{(2)}\right)^{2}\right] \mathrm{q}_{1}, \\
\mathcal{D C} \mathcal{C}^{(1)}\left(\mathrm{n}^{(0)}\right) \cdot \mathrm{n}^{(2)} & =K A^{\prime}(u) \sigma^{(2)} \mathrm{q}_{1} .
\end{array}
$$

- Consistency: Setting $q_{0} \cdot g^{(3)}=0$ yields the $\mathcal{O}\left[\delta^{3}\right]$ consistency condition,

$$
\frac{\partial}{\partial t} \sigma^{(1)}+c \frac{\partial}{\partial x}\left(A^{\prime}(u) \sigma^{(1)}\right)=\nu \frac{\partial^{2}}{\partial x^{2}} \sigma^{(1)} \text {. }
$$

Note that as with $L B_{1}$ and $L B_{2}$, the $\mathcal{O}\left[\delta^{3}\right]$ consistency condition is satisfied with $\sigma^{(1)}(x ; t)=$ const. Let us choose $\sigma^{(1)}(x ; t)=0$; further analysis reflects this choice.

- Solve for $\mathrm{n}^{(3)}$ : We have

$$
\begin{aligned}
\mathbf{n}^{(3)} & =\mathcal{L}^{+} \cdot \mathbf{g}^{(3)}+\sigma^{(2)} \mathbf{q}_{0} \\
& =\sum_{k=0}^{1} c_{k}^{(3)} \mathbf{q}_{k},
\end{aligned}
$$

where

$$
\begin{aligned}
c_{0}^{(3)} & =\sigma^{(3)} \\
c_{1}^{(3)} & =-\frac{L^{3}}{3} u_{x x x}+K\left(\frac{L^{2}}{2} \frac{\partial}{\partial x}+T \frac{\partial}{\partial t}\right) A^{\prime}(u)+L \sigma_{x}^{(2)}-6 K A^{\prime}(u) \sigma^{(2)} \\
& +K\left[\left(\sigma^{(2)}\right)^{2}-\left(L u_{x}-K A(u)\right)^{2}\right]
\end{aligned}
$$


in which $\sigma^{(3)}$ is the arlitsary scalia parameter int doduced by the nullspare of the linearized collision operator.

$\mathcal{O}\left[\delta^{4}\right]$. We find

$$
\begin{aligned}
& \mathcal{L} \cdot 1^{(4)} \\
& =\left[\frac{L^{4}}{4 !}\left(\vec{e}_{k} \cdot \nabla\right)^{4}+\frac{L^{2} T}{2}\left(\vec{e}_{k} \cdot \nabla\right)^{2} \frac{\partial}{\partial t}+\frac{T^{2}}{2} \frac{\partial^{2}}{\partial t^{2}}\right] \mathbf{n}^{(0)} \\
& +\left[\frac{L^{3}}{3 !}\left(\vec{c}_{k} \cdot \nabla\right)^{3}+L T\left(\vec{c}_{k} \cdot \nabla\right) \frac{\partial}{\partial !}\right] \mathrm{n}^{(1)}+\left[\frac{L^{2}}{2 !}\left(\vec{c}_{k} \cdot \nabla\right)^{2}+T \frac{\partial}{\partial t}\right] \mathrm{n}^{(2)} \\
& +\left[L\left(\vec{c}_{k} \cdot \nabla\right)\right] n^{(3)}-\frac{1}{2 \cdot 1} \mathcal{D}^{4} \mathcal{C}^{(0)}\left(n^{(1)}\right) \cdot n^{(1)} n^{(1)} n^{(1)} n^{(1)} \\
& -\frac{1}{2} \mathcal{D}^{3} \mathcal{C}^{(0)}\left(n^{(0)}\right) \cdot n^{(1)} n^{(1)} n^{(2)}-\mathcal{D}^{2} \mathcal{C}^{(0)}\left(n^{(0)}\right) \cdot n^{(1)} n^{(3)}-\frac{1}{2} \mathcal{D}^{2} \mathcal{C}^{(0)}\left(n^{(0)}\right) \cdot n^{(2)} n^{(2)} \\
& -\frac{1}{6} \mathcal{D}^{9} \mathcal{C}^{(1)}\left(n^{(0)}\right) \cdot n^{(1)} n^{(1)} n^{(1)}-\mathcal{D}^{2} \mathcal{C}^{(1)}\left(\mathbf{n}^{(0)}\right) \cdot n^{(1)} n^{(2)}-\mathcal{D} \mathcal{C}^{(1)}\left(n^{(0)}\right) \cdot n^{(3)} \\
& \equiv g^{(4)}
\end{aligned}
$$

where, using Eq. 3.34,

$$
\begin{aligned}
\tilde{c}_{0}^{(4)} & =T \sigma_{t}^{(2)}+6 K L \frac{\partial}{\partial x}\left(A^{\prime}(u) \sigma^{(2)}\right)-\frac{L^{2}}{2} \sigma_{x x}^{(2)} \\
& +T \mathcal{F}\left(K, L ; x, t ; u, u_{x}, u_{x x}, u_{x x x x} . u_{x x x x}\right), \\
\tilde{c}_{1}^{(4)} & =L \sigma_{x}^{(3)}-K A^{\prime}(u) \sigma^{(3)},
\end{aligned}
$$

for

$$
\begin{aligned}
& \mathcal{F}\left(K, L, T ; x, t ; u, u_{x}, u_{x x}, u_{x x x}, u_{x x x x}\right) \\
& \equiv+\frac{L^{4}}{12 T} u_{x x x x}+\frac{K L^{3}}{T}\left[\frac{1}{3} \frac{\partial^{3}}{\partial x^{3}} A(u)+u_{x} u_{x x}\right]-\frac{K^{2} L^{2}}{T}\left[\frac{2}{3} \frac{\partial^{2}}{\partial x^{2}}\left(A(u) A^{\prime}(u)\right)-\frac{1}{6} u_{x x}\right] \\
& +\frac{2 K^{3} L}{T} A(u) A^{\prime}(u) u_{x},
\end{aligned}
$$


and in which

$$
\begin{array}{ll}
\mathcal{D}^{4} \mathcal{C}^{(0)}\left(\mathbf{n}^{(0)}\right) \cdot \mathbf{n}^{(1)} \mathbf{n}^{(1)} \mathbf{n}^{(1)} \mathbf{n}^{(1)} & =0, \\
\mathcal{D}^{3} \mathcal{C}^{(0)}\left(\mathbf{n}^{(0)}\right) \cdot \mathbf{n}^{(1)} \mathbf{n}^{(1)} \mathbf{n}^{(2)} & =0, \\
\mathcal{D}^{2} \mathcal{C}^{(0)}\left(\mathbf{n}^{(0)}\right) \cdot \mathbf{n}^{(1)} \mathbf{n}^{(3)} & =0, \\
\mathcal{D}^{2} \mathcal{C}^{(0)}\left(\mathbf{n}^{(0)}\right) \cdot \mathbf{n}^{(2)} \mathbf{n}^{(2)} & =0, \\
\mathcal{D}^{3} \mathcal{C}^{(1)}\left(\mathbf{n}^{(0)}\right) \cdot \mathbf{n}^{(1)} \mathbf{n}^{(1)} \mathbf{n}^{(1)} & =0, \\
\mathcal{D}^{2} \mathcal{C}^{(1)}\left(\mathbf{n}^{(0)}\right) \cdot \mathbf{n}^{(1)} \mathbf{n}^{(2)} & =0, \\
\mathcal{D} \mathcal{C}^{(1)}\left(\mathbf{n}^{(0)}\right) \cdot \mathbf{n}^{(3)} & =K A^{\prime}(u) \sigma^{(3)} .
\end{array}
$$

Note that our choice of $\sigma^{(1)}(x ; t)=0$ played a significant role in simplifying the above calculations.

- Consistency: Setting $\mathrm{q}_{0} \cdot \mathrm{g}^{(4)}=0$ yields the $\mathcal{O}\left[\delta^{4}\right]$ consistency condition,

$$
\begin{aligned}
\frac{\partial}{\partial t} \sigma^{(2)}+6 c \frac{\partial}{\partial x}\left(A^{\prime}(u) \sigma^{(2)}\right) & =\nu \frac{\partial^{2}}{\partial x^{2}} \sigma^{(2)} \\
& -\mathcal{F}\left(K, L, T ; x, t ; u, u_{x}, u_{x x}, u_{x x x}, u_{x: x x}\right)
\end{aligned}
$$

Note that Eq. 3.41 may be written

$$
(\mathbf{L}+\mathbf{h})\left[\sigma^{(2)}\right]=\mathcal{F}
$$

where operator

$$
\mathrm{L} \equiv a(x ; t) \frac{\partial^{2}}{\partial x^{2}}+b(x ; t) \frac{\partial}{\partial x}-\frac{\partial}{\partial t}
$$

in which

$$
\begin{aligned}
& a(x ; t)=\nu, \\
& b(x ; t)=-6 c A^{\prime}(u), \\
& \mathbf{h}(x ; t)=12 c u_{x} .
\end{aligned}
$$

Note that $L$ is uniformly parabolic in region $E_{T}=([0, L] \times[0, L]) \times(0, T]$. Suppose that $u \in C^{4}([0,1],[0,1])$. Then under this and additional assumptions 
(regarding initial and boundary conditions) and regularity anguments, it can be shown [5.t] that solutions $\sigma^{(2)}$ of Eq. 3.12 ane $\left(^{2}(\pi \cdot \pi)\right.$ functions and, in particular, $\sigma^{(2)}, \sigma_{x^{2}}^{(2)}$, and $\sigma_{x x}^{(2)}$ are uniformly bounded. We will assume that such bounds exist to obtain statements regarding numerical convergence of the lattice method (see Lemma 3.9 for how the bounds apply to numerical consistency and Lemma 3.14 for how they apply to continuum maximum and minimum principles for the truncated equilibrium expansion).

- Solve for $\mathrm{n}^{(4)}$ : We have

$$
\begin{aligned}
\mathbf{n}^{(4)} & =\mathcal{L}^{+} \cdot \mathbf{g}^{(4)}+\sigma^{(2)} \mathbf{q}_{0} \\
& =\sum_{k=0}^{1} c_{k}^{(4)} \mathbf{q}_{k},
\end{aligned}
$$

where

$$
\begin{aligned}
& c_{0}^{(4)}=\sigma^{(4)}, \\
& c_{1}^{(4)}=K A^{\prime}(u) \sigma^{(3)}-L \sigma_{x}^{(3)},
\end{aligned}
$$

in which $\sigma^{(4)}$ is the arbitrary scalar parameter introduced by the nullspace of the linearized collision operator.

$\mathcal{O}\left[\delta^{5}\right]$ : At this order, only the consistency condition is to be determined. This condition specifies $\sigma^{(3)}$. We will fiad that $\sigma^{(3)}(x ; t)=$ const. satisfies the condition. We find

$$
\begin{aligned}
& \mathcal{L} \cdot \mathbf{n}^{(5)} \\
& =\left[\frac{L^{5}}{5 !}\left(\vec{e}_{k} \cdot \nabla\right)^{5}+\frac{L T^{2}}{2}\left(\vec{e}_{k} \cdot \nabla\right) \frac{\partial^{2}}{\partial t^{2}}+\frac{L^{3}}{6}\left(\vec{e}_{k} \cdot \nabla\right)^{3} \frac{\partial}{\partial t}\right] \mathrm{n}^{(0)}
\end{aligned}
$$




$$
\begin{aligned}
& +\left[\frac{L^{4}}{4 !}\left(\vec{e}_{k} \cdot \nabla\right)^{4}+\frac{L^{2} T}{2}\left(\vec{e}_{k} \cdot \nabla\right)^{2} \frac{\partial}{\partial t}+\frac{T^{2}}{2} \frac{\partial^{2}}{\partial t^{2}}\right] \mathbf{n}^{(1)} \\
& +\left[\frac{L^{3}}{3 !}\left(\vec{e}_{k} \cdot \nabla\right)^{3}+L T\left(\vec{e}_{k} \cdot \nabla\right) \frac{\partial}{\partial t}\right] \mathbf{n}^{(2)} \\
& +\left[\frac{L^{2}}{2 !}\left(\vec{e}_{k} \cdot \nabla\right)^{2}\right] \mathbf{n}^{(3)}+\left[L\left(\vec{e}_{k} \cdot \nabla\right)\right] \mathbf{n}^{(4)} \\
& +(\text { Coll. }) \\
& \equiv \mathbf{g}^{(5)} \\
& =\sum_{k=0}^{1} \tilde{c}_{k}^{(5)} \mathbf{q}_{k},
\end{aligned}
$$

where

$$
\tilde{c}_{0}^{(5)}=K L \frac{\partial}{\partial x}\left(A^{\prime}(u) \sigma^{(3)}\right)-\frac{L^{2}}{2} \sigma_{x x}^{(3)}+T \sigma_{t}^{(3)} .
$$

and (Coll.) is the contribution from the coefficient of $\delta^{5}$ in the collision operator expansion, Eq. 2.18. ${ }^{14}$ Note that (Coll.) is orthogonal to the nullspace of the linearized collision operator, and therefore does not contribute to the consistency condition. The remaining coefficient, $\tilde{c}_{1}^{(5)} ;$ left uncalculated..$^{15}$

- Consistency: Setting $\mathrm{q}_{0} \cdot \mathrm{g}^{(5)}=0$ yields the $\mathcal{O}\left[\delta^{5}\right]$ consistency condition,

$$
\frac{\partial}{\partial t} \sigma^{(3)}+c \frac{\partial}{\partial x}\left(A^{\prime}(u) \sigma^{(3)}\right)=\nu \frac{\partial^{2}}{\partial x^{2}} \sigma^{(3)}
$$

Note that $\sigma^{(3)}(x ; t)=$ const. satisfies Eq. 3.43. Let us choose $\sigma^{(3)}(x ; t)=0$. (This implies that $\check{c}_{1}^{(-)}=0$.)

\footnotetext{
${ }^{14}($ Coll. $)$ consists of the $\mathcal{O}\left[\delta^{5}\right]$ collision operator expansion terms minus $\mathcal{L} \cdot \mathbf{n}^{(5)}$.

${ }^{15} \tilde{c}_{1}^{(5)}$ would need to be calculated for determining $\mathbf{n}^{(5)}$, which would also mean calculating the (Coll.) term. One would complete such calculations to determine the $\mathcal{O}\left[\delta^{6}\right]$ consistency condition.
} 
- Solve for $\mathrm{n}^{(5)}$ : We would complete this calculation were we to desire the (') $\left[f^{(i)}\right.$ consistency condition, which would determine, $\sigma^{(4)}$. But since $\sigma^{(4)}$ does not. appear in the truncated e(pulibrium expansion, which is used in establishing consistency (as per the noxt subsection), it is not necessary to determine $\sigma^{(4)}$ nor, hence, $n^{(5)}$.

This completes the first application of the discrete Chapman-Enskog procedure. It determined hydrodynamical equation of the lattice Boltzmann method to be Eq. 3.3.4 and the consistency equations for $\sigma^{(1)}, \sigma^{(2)}$, and $\sigma^{(3)}$ to be Eys. 3.37, 3.41, and 3.43, respectively. We saw that $\sigma^{(1)}$ and $\sigma^{(3)}$ may be chosen to be constants, and that $u=\sigma^{(0)} \in C^{4}([0,1],[0,1])$ and $\sigma^{(2)} \in C^{2}(\mathcal{R}, \mathcal{R})$.

\subsubsection{Coirvergence}

To obtain a statement regarding the convergence of the lattice Boltzmann method, we establish consistency, discrete and continuum maximum and minimum principles, and stability. The discrete maximum and minimum principles result from monotonicity arguments.

Lemma 3.9 (Consistengy of LB Method for Burgers' Equation). Define the truncated equilibrium expansion by

$$
\mathbf{h}=\sum_{j=0}^{3} \delta^{j} \mathbf{h}^{(j)}
$$

in which $\mathrm{h}^{(j)} \equiv \mathrm{n}^{(j)}$, the $\mathrm{n}^{(j)}$ as defined by Eqs. 3.32, 3.33, 3.36, and 3.38 in the application of the discrete Chapman-Enstog procedure of Section 3.2.7. Then hatisfies

$$
\mathcal{A} \mathrm{h}=\mathrm{h}+\mathcal{C}(\mathrm{h})-\mathcal{T}(\mathrm{h})
$$


in whuch truncation error, $\mathcal{T}(\mathrm{h})=\mathcal{O}\left[\delta^{5}\right]$. Suppose $\sigma^{(0)} \in C^{4}([0,1],[0,1])$ and $\sigma^{(2)} \in$ $C^{2}(\mathcal{R}, \mathcal{R}){ }^{16}$ Let $\Delta t=T \delta^{2}, \Delta x=L \delta, \nu=L^{2} /(2 T)$, and $c=K L / T$, for spatial, temporal, and advection scale lengths $L, T$, and $K$, respectively. Then

$$
\lim _{\Delta t \rightarrow 0} \frac{1}{\Delta t}\|\mathcal{T}(\mathbf{h})\|=0
$$

for some norm, $\|\cdot\|$.

Proof. First, we apply the discrete Chapman. Enskog procedure to determine the truncated equilibrium expansion. Then we determine the remainder, $T(h)$. Finally, we show that $\lim _{\Delta t \rightarrow 0} \frac{1}{\Delta t}\|T(h)\|=0$.

\section{Part 1: Determining the Truncated Equilibrium Expansion}

Recall that $\sigma^{(1)}(x ; t)=$ const. and $\sigma^{(3)}(x ; t)=$ const. satisfy the $\mathcal{O}\left[\delta^{3}\right]$ and $\mathcal{O}\left[\delta^{5}\right]$ consistency conditions, i.e., Eq. 3.37 and Eq. 3.43, respectively. Choose $\sigma^{(1)}(x ; t)=$ $\sigma^{(3)}(x ; t)=0$. Then using that $h^{(\jmath)} \equiv n^{(\jmath)}$ and Eqs. 3.32, 3.33, 3.36, and 3.38 for $n^{(0)}, n^{(1)}, n^{(2)}$, and $n^{(3)}$, respectively, we find

$$
\begin{gathered}
\mathrm{n}^{(0)}=u \mathrm{q}_{0} \\
\mathrm{~h}^{(1)}=\sum_{k=0}^{1} c_{k}^{(1)} \mathrm{q}_{k}, \mathrm{~h}^{(2)}=\sum_{k=0}^{1} c_{k}^{(2)} \mathrm{q}_{k}, \mathrm{~h}^{(3)}=\sum_{k=0}^{1} c_{k}^{(3)} \mathrm{q}_{k},
\end{gathered}
$$

where

$$
\begin{aligned}
& c_{0}^{(1)}=0, \\
& c_{1}^{(1)}=K A(u)-L u_{x} ;
\end{aligned}
$$

${ }^{16}$ Recall that $u(x ; t) \equiv \sigma^{(0)}(x ; t)$. 


$$
\begin{aligned}
& \text { (1) }=\sigma^{(2)} \text {. } \\
& c_{1}^{(2)}=l i \Lambda^{\prime}(11) \sigma^{(1)}-l, \sigma_{x}^{(1)} ; \\
& c_{(1)}^{(3)}=0 \text {, } \\
& ()^{(3)}=-\frac{L^{3}}{3} u_{x x x}+K\left(\frac{L^{2}}{2} \frac{\partial}{\partial x}+\Gamma \frac{\partial}{\partial t}\right) A^{\prime}(u)+L \sigma_{x}^{(2)}-6 K A^{\prime}(u) \sigma^{(2)} \\
& +K\left[\left(\sigma^{(2)}\right)^{2}-\left(L u_{x}-K A(u)\right)^{2}\right] \text {. }
\end{aligned}
$$

\section{Part 2: Determining the Remainder}

Now, to determine the remainder, $\mathcal{T}(\mathrm{h})$, let us apply the discrete Chapman-Linkog procedure to h. We apply the advection and collision operator expansions(Eq. 2.24 and $E_{1} .2 .18$, respectively) to the truncated equilibrium expansion, h. Care is taken in noting that only up to the $\mathcal{O}\left[\delta^{3}\right]$ terms of $\mathbf{n}$ appear in $\mathbf{h}$. Thus, some of the terms in the advection and collision operator expansions do not appear in the discrete Chapman-Enskog expansion on $\mathbf{h}$. The resulting expansion follows:

$$
\mathcal{A} \mathrm{h}-\mathrm{h}-\mathcal{C}(\mathrm{h})=-\mathcal{T}(\mathrm{h})=-\sum_{j=0}^{4} \delta^{j} \mathrm{~T}^{(j)}+\mathcal{O}\left[\delta^{5}\right]
$$

where

$$
\begin{aligned}
\mathbf{T}^{(0)} & =\mathcal{C}^{(0)}\left(\mathrm{h}^{(0)}\right) \\
\mathrm{T}^{(1)} & =\mathcal{L} \cdot \mathrm{h}^{(1)}+\mathcal{C}^{(1)}\left(\mathrm{h}^{(0)}\right)-L\left[\vec{e}_{k} \cdot \nabla\right] \mathrm{h}^{(0)}, \\
\mathrm{T}^{(2)} & =\mathcal{L} \cdot \mathrm{h}^{(2)}+\frac{1}{2} \mathcal{D}^{2} \mathcal{C}^{(0)}\left(\mathrm{h}^{(0)}\right) \cdot \mathrm{h}^{(1)} \mathrm{h}^{(1)}+\mathcal{L} \mathcal{C}^{(1)}\left(\mathrm{h}^{(0)}\right) \cdot \mathrm{h}^{(1)} \\
& -\frac{L^{2}}{2}\left[\left(\vec{e}_{k} \cdot \nabla\right)^{2}\right] \mathrm{h}^{(0)}-T \frac{\partial}{\partial t} \mathrm{~h}^{(0)}+L\left[\overrightarrow{e_{k}} \cdot \nabla\right] \mathrm{h}^{(1)}, \\
\mathrm{T}^{(3)} & =\mathcal{L} \cdot \mathrm{h}^{(3)}+\frac{1}{6} \mathcal{D}^{3} \mathcal{C}^{(0)}\left(\mathrm{h}^{(0)}\right) \cdot \mathrm{h}^{(1)} \mathrm{h}^{(1)} \mathrm{h}^{(1)}+\mathcal{D}^{2} \mathcal{C}^{(0)}\left(\mathrm{h}^{(0)}\right) \cdot \mathrm{h}^{(1)} \mathrm{h}^{(2)} \\
& +\frac{1}{2} \mathcal{D}^{2} \mathcal{C}^{(1)}\left(\mathrm{h}^{(0)}\right) \cdot \mathrm{h}^{(1)} \mathrm{h}^{(1)}+\left(\mathcal{D} \mathcal{D} \mathcal{C}^{(1)}\left(\mathrm{h}^{(0)}\right) \cdot \mathfrak{h}^{(2)}\right.
\end{aligned}
$$




\section{P'urt is shouring Comsistenc!l}

Ihe combined results of parts 1 and 2 establish consistences as summarized by the following: Note that the $h(3), j \in\{0,1,2,3\}$, are miformly bounded since

$$
\sigma^{(0)} \in(\cdot 1)([0,1),[0,1]) \text { and } \sigma^{(2)} \in\left(!^{2}(\pi, \pi)\right.
$$

by the assumptions of the lenuma, Hence, $\exists$ a norm, $\|\cdot\|$, such that $\lim _{\Delta t \rightarrow(1)} \frac{1}{\Delta t}\|T(h)\|=$ (1) by Theorem 2.47 ,

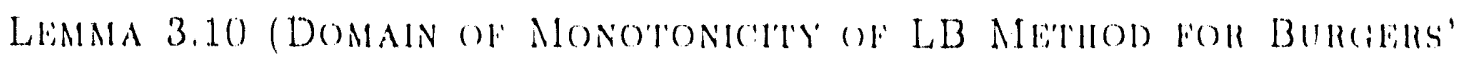
Equation). Lete $E(0,1)$ in the lattice Boltzmann method.

$$
A n_{i}^{n}=n_{i}^{n}+C\left(n_{1}^{n}\right)
$$

where:

$$
\mathcal{C}\left(\mathbf{n}_{i}^{n}\right)=q_{1}\left[\frac{1}{2}\left(-\left(n_{0}\right)_{i}^{n}+\left(n_{1}\right)_{i}^{n}\right)+\frac{\epsilon}{2}\left(\left(n_{0}\right)_{i}^{n}+\left(n_{1}\right)_{i}^{n}-2\left(n_{0}\right)_{i}^{n}\left(n_{1}\right)_{i}^{n}\right)\right]
$$

and

$$
\mathcal{A} n_{i}^{n}=\left[\begin{array}{l}
\left(n_{1}\right)_{i-1}^{n+1} \\
\left(n_{1}\right)_{i+1}^{n+1}
\end{array}\right] .
$$

Let $\mathcal{E}^{(0)}:=\mathcal{E}^{(1)}=[0,1]$. Then $\mathcal{E} \equiv \mathcal{E}^{(0)} \times \mathcal{E}^{(1)}$ is the domain of monotonicity for the method.

I'roof. There are three parts to proving the lemma: (1) show that the conditions of monotonicity are satisfied in $\mathcal{E},(2)$ show that $\mathcal{E}$ cannot be extended to a larger connected region, and (3) show that $\mathcal{E}$ has volume. (learly, (2) and (3) are true.17

for (2) let us write the Lattice Boltzinann liquation, lide. 3.48, in the form

$$
\mathrm{n}_{i}^{n+1}=\left[\begin{array}{l}
U_{0}\left(\mathrm{n}_{i-1}^{n}\right) \\
H_{1}\left(\mathrm{n}_{i+1}^{n}\right)
\end{array}\right],
$$

\footnotetext{
$17 \mathcal{E}$ camnot be extended beyond $[0,1]^{\prime \prime}$, and it obviously has volume.
} 
Part 3: Showing Consistency

The combined results of parts 1 and 2 establish consistency, as summarized by the following: Note that the $\mathrm{h}^{(j)}, j \in\{0,1,2,3\}$, are uniformly bounded since

$$
\sigma^{(0)} \in C^{4}(\{0,1],[0,1]) \text { and } \sigma^{(2)} \in C^{2}(\mathcal{R}, \mathcal{R})
$$

by the assumptions of the lemma. Hence, $\exists$ a norm, $\|\cdot\|$, such that $\lim _{\Delta t \rightarrow 0} \frac{1}{\Delta t}\|\mathcal{T}(\mathbf{h})\|=$ 0 by Theorem 2.47 .

Lemma 3.10 (Domain of Monotonicity of LB Method for Burgers' Equation). Let $\epsilon \in(0,1)$ in the lattice Boltzmann method.

$$
\mathcal{A} \mathbf{n}_{i}^{n}=\mathbf{n}_{i}^{n}+\mathcal{C}\left(\mathbf{n}_{i}^{n}\right)
$$

where

$$
\mathcal{C}\left(\mathbf{n}_{i}^{n}\right)=\mathbf{q}_{1}\left[\frac{1}{2}\left(-\left(n_{0}\right)_{i}^{n}+\left(n_{1}\right)_{i}^{n}\right)+\frac{\epsilon}{2}\left(\left(n_{0}\right)_{i}^{n}+\left(n_{1}\right)_{i}^{n}-2\left(n_{0}\right)_{i}^{n}\left(n_{1}\right)_{i}^{n}\right)\right]
$$

and

$$
\mathcal{A} \mathbf{n}_{i}^{n}=\left[\begin{array}{c}
\left(n_{0}\right)_{i-1}^{n+1} \\
\left(n_{1}\right)_{i+1}^{n+1}
\end{array}\right] .
$$

Let $\mathcal{E}^{(0)}=\mathcal{E}^{(1)}=[0,1]$. Then $\mathcal{E} \equiv \mathcal{E}^{(0)} \times \mathcal{E}^{(1)}$ is the domain of monotonicity for the method.

Proof. There are three parts to proving the lemma: (1) show that the conditions of monotonicity are satisfied in $\mathcal{E},(2)$ show that $\mathcal{E}$ cannot be extended to a larger connected region, and (3) show that $\mathcal{E}$ has volume. Clearly, (2) and (3) are true. ${ }^{17}$

For (2) let us write the Lattice Boltzmann Equation, Eq. 3.48, in the form

$$
\mathbf{n}_{i}^{n+1}=\left[\begin{array}{l}
H_{0}\left(\mathbf{n}_{i-1}^{n}\right) \\
H_{1}\left(\mathbf{n}_{i+1}^{n}\right)
\end{array}\right],
$$

\footnotetext{
${ }^{17} \mathcal{E}$ cannot be extended beyond $[0,1]^{2}$, and it obviously has volume.
} 
where

$$
\left[\begin{array}{l}
H_{0}\left(\mathbf{n}_{i-1}^{n}\right) \\
H_{1}\left(\mathbf{n}_{i+1}^{n}\right)
\end{array}\right]=\left[\begin{array}{l}
\frac{1+\epsilon}{2}\left(\left(n_{0}\right)_{i-1}^{n}+\left(n_{1}\right)_{i-1}^{n}\right)-\epsilon\left(n_{0}\right)_{i-1}^{n}\left(n_{1}\right)_{i-1}^{n} \\
\frac{1-\epsilon}{2}\left(\left(n_{0}\right)_{i+1}^{n}+\left(n_{1}\right)_{i+1}^{n}\right)+\epsilon\left(n_{0}\right)_{i+1}^{n}\left(n_{1}\right)_{i+1}^{n}
\end{array}\right] .
$$

Define $G_{k} \equiv \frac{\partial}{\partial \mathbf{n}_{i-e_{k}}^{n}} H_{k}\left(\mathbf{n}_{i-\vec{e}_{k}}^{n}\right), k \in\{0,1\}$. We must show that $G_{k} \geq 0$ for $k \in\{0,1\}$. Well, we have

$$
\left[\begin{array}{l}
G_{0} \\
G_{1}
\end{array}\right]=\left[\begin{array}{ll}
\frac{1+\epsilon}{2}-\epsilon\left(n_{1}\right)_{i-1}^{n} & \frac{1+\epsilon}{2}-\epsilon\left(n_{0}\right)_{i-1}^{n} \\
\frac{1-\epsilon}{2}+\epsilon\left(n_{1}\right)_{i+1}^{n} & \frac{1-\epsilon}{2}+\epsilon\left(n_{0}\right)_{i+1}^{n}
\end{array}\right] \geq 0, \forall i \in \mathfrak{L}
$$

if

$$
\begin{aligned}
& -\frac{1-\epsilon}{2 \epsilon} \leq\left(n_{0}\right)_{i}^{n} \leq \frac{1+\epsilon}{2 \epsilon}, \\
& -\frac{1-\epsilon}{2 \epsilon} \leq\left(n_{1}\right)_{i}^{n} \leq \frac{1+\epsilon}{2 \epsilon},
\end{aligned}
$$

which is certainly true since $\epsilon \in(0,1)$. Hence, the domain of monotonicity is $\mathcal{E}$.

We have established that the domain of monotonicity encompasses all possible occupation numbers, i.e., $n_{k} \in[0,1]$. Hence, the method is monotonically increasing.

Lemma 3.11 (Discrete Maximum/Minimum Principle of LB Method FOR BURGERS' EQUATION). Theorem 2.49 holds for the lattice Boltzmann method, Eq. 3.48, with domain of monotonicity, $\mathcal{E}=[0,1]^{2}$.

Proof. First note that any initial condition in this method must necessarily ${ }^{18}$ be in $\mathcal{E}$. Let $\mathrm{M}_{ \pm}$denote the extreme values of $\mathcal{E}$, i.e.,

$$
\mathbf{M}_{-}=\left[\begin{array}{l}
0 \\
0
\end{array}\right] \text { and } \mathbf{M}_{+}=\left[\begin{array}{l}
1 \\
1
\end{array}\right]
$$

Then note that $\mathcal{C}\left(\mathbf{M}_{-}\right)=\mathcal{C}\left(\mathbf{M}_{+}\right)=0 \in[0,1]^{2}$.

\footnotetext{
${ }^{18}$ By definition of a lattice Boltzmann method with the Fermi Exclusion Principle.
} 
To establish a continuum maximum/minimum principle on h, we use two lemmas. One is a maximum/minimum principle for Eq. 3.29. The other is a maximum/minimum principle for the hydrodynamical equation of our lattice method, Eq. 3.34 .

Lemma 3.12 (Maximum/Minimual Principle for the 1-D Viscous BurgERS EQUATION). Let $\rho(x ; t)$ satisfy the one-dimensional viscous Burgers equation, Eq. 3.29, on $\Omega=[0, L] \times(0, T]$ with $\nu>0$, periodic boundary conditions and initial condition $\rho(x ; 0)=\rho_{I}(x) \in C^{2}(\mathcal{R}, \mathcal{R})$. Then the maximum and minimum values of $\rho(x ; t)$ occur at the initial boundary.

Proof. We easily verify that Eq. 3.29 is parabolic for all functions $\rho$. Noting that any constant satisfies Eq. 3.29, we may apply Theorem 12 of $[63$, p. 187] to conclude that the maximum and minimum values of $\rho(x ; t)$ occur at the initial boundary.

Corollary 3.13. Let $u(x ; t)$ be a solution of Eq. 3.34 on $\Omega=[0, L] \times\left(0,1^{\prime}\right]$ with initial condition $u(x ; 0)=u_{I}(x) u_{I} \in C^{2}(\mathcal{R}, \mathcal{R})$ satisfying $0 \leq \tilde{R}_{-} \leq u_{I}(x) \leq \tilde{R}_{+} \leq 1$ and periodic boundary conditions. Then the maximum and minimum values of $u(x ; t)$ occur at the initial time, i.e., $u(x ; t) \in\left[\tilde{R}_{-}, \tilde{R}_{+}\right], \forall(x ; t) \in[0, L] \times[0, T]$.

Proof. Let $\rho(x ; t)=\frac{K L}{T}(1-2 u)$ and $\nu=\frac{L^{2}}{2 T}$. Under this transformation, Lemma 3.12 may be applied to obtain the desired result.

Lemma 3.14 (Continuum Maximum/Minimum Principle of LB Metiod For Burgers' Equation). Let $u(x ; t) \in C^{4}(\mathcal{R}, \mathcal{R})$ be a solution of Eq. 3.34. Let $\mathrm{h}^{(j)}, j \in\{0,1,2,3\}$ be defined by Eqs. 3.46-3.47. Then suppose $\exists$ constants $R_{-}, R_{+}$, $B^{(1)}, B^{(2)}$, and $B^{(3)}$ such that

1. $0 \leq R_{-}<u(x ; 0)<R_{+} \leq 1, \forall x \in[0, L]$, and

2. For each $j \in\{1,2,3\},\left\|\mathbf{h}^{(j)}\right\|_{\ell_{\infty}} \leq B^{(j)}, \forall(x ; t) \in[0, L] \times(0, T]$. 
Then $\exists \delta_{0}>0 \ni$ for $\delta \in\left(0, \delta_{0}\right)$

$$
\left\|\mathrm{h}^{n}-\frac{R_{+}+R_{-}}{2}\right\|_{\ell_{\infty}} \leq \frac{R_{+}-R_{-}}{2}
$$

for all time steps $n \geq 0$.

Proof. Let $\tilde{R}_{-}=\inf u(x ; 0)$ and $\tilde{R}_{+}=\sup u(x ; 0)$. Then by Corollary 3.13 and noting that $R_{-}<\tilde{R}_{-} \leq \tilde{R}_{+}<R_{+}$, we have

$$
\left\|\mathrm{h}^{(0)}-\frac{R_{+}+R_{-}}{2}\right\|_{\ell_{\infty}}<\frac{R_{+}-R_{-}}{2}
$$

where $h_{0}^{(0)}=h_{1}^{(0)}=u(x ; t)$. Consequently,

$$
R_{-}<\left(h_{k}^{(0)}\right)_{i}^{n}<R_{+}
$$

for all lattice nodes $i$, time steps $n$, and directions $k \in\{0,1\}$.

Now, choose $\delta_{0} \ni R_{-} \leq\left(h_{k}^{(0)}\right)_{i}^{0}+\sum_{j=1}^{3}\left(\delta_{0}\right)^{j} B^{(j)} \leq R_{+}$for all lattice nodes $i$ and directions $k \in\{0,1\} .^{19}$ Then

$$
\begin{aligned}
\left\|\mathrm{h}^{n}-\frac{R_{+}+R_{-}}{2}\right\|_{\ell_{\infty}} & =\left\|\sum_{j=0}^{3} \delta^{j}\left(\mathrm{~h}^{(j)}\right)^{n}-\frac{R_{+}+R_{-}}{2}\right\|_{\ell_{\infty}} \\
& \leq\left\|\left(\mathrm{h}^{(0)}\right)^{n}-\frac{R_{+}+R_{-}}{2}\right\|_{\ell_{\infty}}+\sum_{j=1}^{3} \delta^{j}\left\|\left(\mathrm{~h}^{(j)}\right)^{n}\right\|_{\ell_{\infty}} \\
& \leq\left\|\left(\mathrm{h}^{(0)}\right)^{n}-\frac{R_{+}+R_{-}}{2}\right\|_{\ell_{\infty}}+\sum_{j=1}^{3}\left(\delta_{0}\right)^{j} B^{(j)} \\
& \leq \frac{R_{+}-R_{-}}{2},
\end{aligned}
$$

the last step by Eq. 3.50 and our choice of $\delta$.

Corollary 3.15. $\exists \delta_{0}>0$ such that $\forall \delta \in\left(0, \delta_{0}\right)$, if $\mathrm{h}_{\vec{i}}^{0} \in \mathcal{E}, \forall \vec{\imath} \in \mathfrak{L}$, then $\mathrm{h}_{\vec{\imath}}^{n} \in \mathcal{E}, \forall \vec{\imath} \in \mathfrak{L} \forall n$, where $\mathcal{E}$ is the domain of monotonicity, $\mathcal{E}=[0,1]^{2}$.

\footnotetext{
${ }^{19}$ This amounts to choosing $\delta_{0} \ni R_{-} \leq u(x ; 9)+\sum_{j=1}^{3}\left(\delta_{0}\right)^{j} B^{(j)} \leq R_{+} \forall x \in[0, L]$ so that Corollary
} 3.13 applies. 
Proof. Let $R_{+} \equiv 1$ and $R_{-} \equiv 0$. Then apply Lemma 3.14 to yield the result.

LemMa 3.16 (Stabilit' of LB Method for Bungers' Equation). Let $\mathrm{n}^{n}, \mathrm{~h}^{n}$ and $L\left[\mathrm{n}^{n}, \mathrm{~h}^{n}\right]$ be defined as in Definition 2.41. Let domain of monotonicity, $\mathcal{E}$, be as in Lemma 3.10. Suppose $\mathrm{n}_{i}^{0},\left(\mathrm{~h}^{(0)}\right)_{i}^{0} \in \mathcal{E}$. And suppose the hypotheses of Lemma 3.14 are satisfied. Then $\exists \tau>0$ such that $\left\|L\left[\mathbf{n}^{n}, \mathrm{~h}^{n}\right]\right\|_{\ell_{1}} \leq 1 \forall n$ such that $0 \leq n \Delta t \leq T$ and $0<\Delta t<\tau$.

Proof. Let $R_{+}=1$ and $R_{-}=0$. Let $\delta_{0}$ be given by Lemma 3.14. Choose $\tau=T\left(\delta_{0}\right)^{2}$ so that $\Delta x \leq L \delta_{0}$. Note that Corollary 3.15 applies to yield that $\mathrm{h}^{n} \in \mathcal{E}$, $\forall n$ such that $0 \leq n \Delta t \leq T$ and $0<\Delta t<\tau$. Then $\left\|L\left[\mathbf{n}^{n}, \mathrm{~h}^{n}\right]\right\|_{\ell_{1}} \leq 1$ by Lemma 2.59 .

Theorem 3.17 (Convergence of LB Method for Burgers' Equation). Let the conditions of Lemmas 3.1, 3.3, and 3.5 be satisfied. Then $\lim _{\Delta t \rightarrow 0} \frac{1}{\Delta t}\left\|F^{n}\right\|_{\ell_{1}}=$ $0, \forall n \ni 0 \leq n \Delta t \leq T$, in which $\Delta t=(\Delta x)^{2} /(2 \nu)$, and $\Delta x=L \delta, \Delta t=T \delta^{2}$, and $\nu=L^{2} /(2 T)$ for spatial and temporal scale lengths $L$ and $T$.

Proof. This is a consequence of Theorem 2.61 .

\section{Discussion}

But since this problem is one-dimensional, some of the abstract quantities that appear in Theorem $2.61 \mathrm{can}$ be easily listed. We include them here for informational purposes.

Let error, $\mathbf{e}_{i}^{n}=\left[\begin{array}{c}\left(e_{0}\right)_{i}^{n} \\ \left(e_{1}\right)_{i}^{n}\end{array}\right]$, be as in Definition 2.44. Then operator $L\left[\mathbf{n}^{n}, \mathrm{~h}^{n}\right]$ can be written in the block diagonal form,

$$
L\left[\mathrm{n}^{n}, \mathrm{~h}^{n}\right]=\operatorname{diag}\left(L_{i}\right)_{i \in \mathfrak{L}}
$$


where $L_{i}$ is a $2 \times 2$ matrix and is given by

$$
L_{i}=\left[\begin{array}{ll}
\frac{1+\epsilon}{2}-\frac{\epsilon}{2}\left[\left(n_{1}\right)_{i}^{n}+\left(h_{1}\right)_{i}^{n}\right] & \frac{1+\epsilon}{2}-\frac{\epsilon}{2}\left[\left(n_{0}\right)_{i}^{n}+\left(h_{0}\right)_{i}^{n}\right] \\
\frac{1-\epsilon}{2}+\frac{\epsilon}{2}\left[\left(n_{1}\right)_{i}^{n}+\left(h_{1}\right)_{i}^{n}\right] & \frac{1-\epsilon}{2}+\frac{\epsilon}{2}\left[\left(n_{0}\right)_{i}^{n}+\left(h_{0}\right)_{i}^{n}\right]
\end{array}\right] .
$$

Indeed

$$
\mathcal{A} \mathbf{e}^{n}=L\left[\mathrm{n}^{n}, \mathrm{~h}^{n}\right] \mathbf{e}^{n}+\mathcal{T}\left(\mathrm{h}^{n}\right) .
$$

The reordered operator matrix, $\tilde{L}\left[\mathbf{n}^{n}, \mathbf{h}^{n}\right]$, as it appears in the proof of Theorem 2.61, can be derived from the recurrence,

$$
\begin{aligned}
\left(e_{0}\right)_{i}^{n+1}= & {\left[\frac{1+\epsilon}{2}-\frac{\epsilon}{2}\left(\left(n_{1}\right)_{i-1}^{n}+\left(h_{1}\right)_{i-1}^{n}\right)\right]\left(e_{0}\right)_{i-1}^{n} } \\
& {\left[\frac{1+\epsilon}{2}-\frac{\epsilon}{2}\left(\left(n_{0}\right)_{i-1}^{n}+\left(h_{0}\right)_{i-1}^{n}\right)\right]\left(e_{1}\right)_{i-1}^{n}+\mathcal{T}_{0}\left(\mathbf{h}_{i-1}^{n}\right) } \\
\left(e_{1}\right)_{i}^{n+1}= & {\left[\frac{1-\epsilon}{2}+\frac{\epsilon}{2}\left(\left(n_{1}\right)_{i+1}^{n}+\left(h_{1}\right)_{i+1}^{n}\right)\right]\left(e_{0}\right)_{i+1}^{n} } \\
& {\left[\frac{1-\epsilon}{2}+\frac{\epsilon}{2}\left(\left(n_{0}\right)_{i+1}^{n}+\left(h_{0}\right)_{i+1}^{n}\right)\right]\left(e_{1}\right)_{i+1}^{n}+\mathcal{T}_{1}\left(\mathbf{h}_{i+1}^{n}\right) . }
\end{aligned}
$$

Note that the coefficients of $\left(e_{k}\right)_{i \neq 1}^{n}$ for $k \in\{0,1\}$ in the r.h.s. of the above are nonnegative by Lemma 3.11 and Lemma 3.14. Hence, $\left\|\tilde{L}\left[\mathbf{n}^{n}, \mathbf{h}^{n}\right]\right\|_{\ell_{1}}=1$.

\section{Summary}

We have shown that the lattice Boltzmann method converges to the solution of Eq. 3.34 in the $L_{1}$-norm. Although the convergence rate for $\mathcal{O}\left[\delta^{3}\right]$ for the error, the method is $\mathcal{O}\left[\delta^{2}\right]$. We see this by the following: Let $U_{i}^{n}=\left(\left(n_{0}\right)_{i}^{n}+\left(n_{1}\right)_{i}^{n}\right) / 2$ be solution computed by the lattice Boltzmann method. Then by consistency arguments (see the expansion for h, Eq. 3.44), $u(x ; t)=\left(h_{0}^{(0)}(x ; t)+h_{1}^{(0)}(x ; t)\right) / 2+\mathcal{O}\left[\delta^{2}\right]$ so that $u_{i}^{n}=\left(\left(h_{0}^{(0)}\right)_{i}^{n}+\left(h_{1}^{(0)}\right)_{i}^{n}\right) / 2+\mathcal{O}\left[\delta^{2}\right]$. We showed that $\mathbf{e}_{i}^{n}=\mathbf{n}_{i}^{n}-\mathbf{h}_{i}^{n}=\mathcal{O}\left[\delta^{3}\right]$ in the 
arguments for convergence. However.

$$
\begin{aligned}
\left(f_{0}\right)_{i}^{n} & =\frac{\mathbf{q}_{0} \cdot \mathbf{e}_{i}^{n}}{2} \\
& =\left(\left(n_{0}\right)_{i}^{n}+\left(n_{1}\right)_{i}^{n}-\left(h_{0}\right)_{i}^{n}-\left(h_{1}\right)_{i}^{n}\right) / 2+\mathcal{O}\left[\delta^{3}\right] \\
& =\left(\left(n_{0}\right)_{i}^{n}+\left(n_{1}\right)_{i}^{n}-\left(h_{0}^{(0)}\right)_{i}^{n}-\left(h_{1}^{(0)}\right)_{i}^{n}+\mathcal{O}\left[\delta^{2}\right]\right)+\mathcal{O}\left[\delta^{3}\right] \\
& =\left(\left(n_{0}\right)_{i}^{n}+\left(n_{1}\right)_{i}^{n}\right) / 2-u_{i}^{n}+\mathcal{O}\left[\delta^{2}\right] .
\end{aligned}
$$

Hence, the lattice method is second-order convergent (and not third-order) because of how we relate the occupation numbers to the exact solution, $u(x ; t)$.

Under the transformation, $P_{i}^{n}=c\left(1-2 U_{i}^{n}\right)$, where $P_{i}^{n} \approx \rho(i \Delta x ; n \Delta t)$ and $\rho(x ; t)$ satisfies Eq. 3.29, we see that the method yields second-order convergent solutions to the one-dimensional viscous Burgers equation. Note that this also verifies convergence of the corresponding lattice gas method to t'ie extent that the covariances may be safely neglected. As previously pointed out Lebowitz, et al. [53] have shown that the covariances indeed can safely be neglected.

\subsection{A Lattice Method for a 2-D Advection-Diff- usion Equation}

This section introduces a lattice Boltzmann method the two-dimensional advectiondiffusion equation,

$$
\rho_{t}+\rho \rho_{x}=\nu\left(\rho_{x x}+\rho_{y y}\right) .
$$

The first section defines the lattice Boltzmann method. The second section derives a hydrodynamical equation (via the discrete Chapman-Enskog procedure) that, under a linear transformation, yields solutions to Eq. 3.51. This provides the groundwork 
for a formal consistency argument of this method. The conditions of monotonicity have yet to be determined. Thus, the convergence proof remains to be completed. However, a numerical study of this method is reported in Section 4.4 and indicates second-order convergence.

\subsubsection{Collision Rules}

First, note the lattice, $\mathfrak{L}$, and velocity vectors, $\vec{e}_{k}, k \in\{0,1,2,3\}$ are the same as those for $\mathrm{LB}_{1}$. Table 3.8 lists the collision rules consistent with our notation; Table 3.7 offers a pictoral description. The rules include an $\mathcal{O}[\delta]$ advection bias so that

$$
a=\frac{1+\epsilon}{2} \text { and } \bar{a}=\frac{1-\epsilon}{2}
$$

where for some constant $K>0$,

$$
\epsilon=K \mathcal{E}
$$

is the advection bias (to the right). As for the lattice method for the one-dimensional Burgers equation (Section 3.2), that $\epsilon=\mathcal{O}[\delta]$ is crucial for recovering the hydrodynamics of the method with the discrete Chapman-Enskog expansion.

\subsubsection{Collision Operator}

By examining the collision rules as listed in Table 3.8, we find the collision operator to be given by

$$
\begin{aligned}
& \mathcal{C}_{0}(\hat{\mathbf{n}})= \\
& \quad+a \overline{\hat{n}_{0}} \overline{\hat{n}_{1}} \hat{n}_{2} \overline{\hat{n}_{3}}+a \overline{\hat{n}_{0}} \overline{\hat{n}_{1}} \hat{n}_{2} \hat{n}_{3}+a \overline{\hat{n}_{0}} \hat{n}_{1} \hat{n}_{2} \overline{\hat{n}_{3}}+a \overline{\hat{n}_{0}} \hat{n}_{1} \hat{n}_{2} \hat{n}_{3} \\
& \quad-\bar{a} \hat{n}_{0} \overline{\hat{n}_{1}} \overline{\hat{n}_{2}} \overline{\hat{n}_{3}}-\bar{a} \hat{n}_{0} \overline{\hat{n}_{1}} \overline{\hat{n}_{2}} \hat{n}_{3}-\bar{a} \hat{n}_{0} \hat{n}_{1} \overline{\hat{n}_{2}} \overline{\hat{n}_{3}}-\bar{a} \hat{n}_{0} \hat{n}_{1} \overline{\hat{n}_{2}} \hat{n}_{3}
\end{aligned}
$$


Table 3.7: Pictorial Description of Collision Rules for Tiwo-Dimensional AdvectionDiffusion. Each line in the table lists possible input states $\alpha$ and corresponding output states $\beta$ with nonzero probabilities.

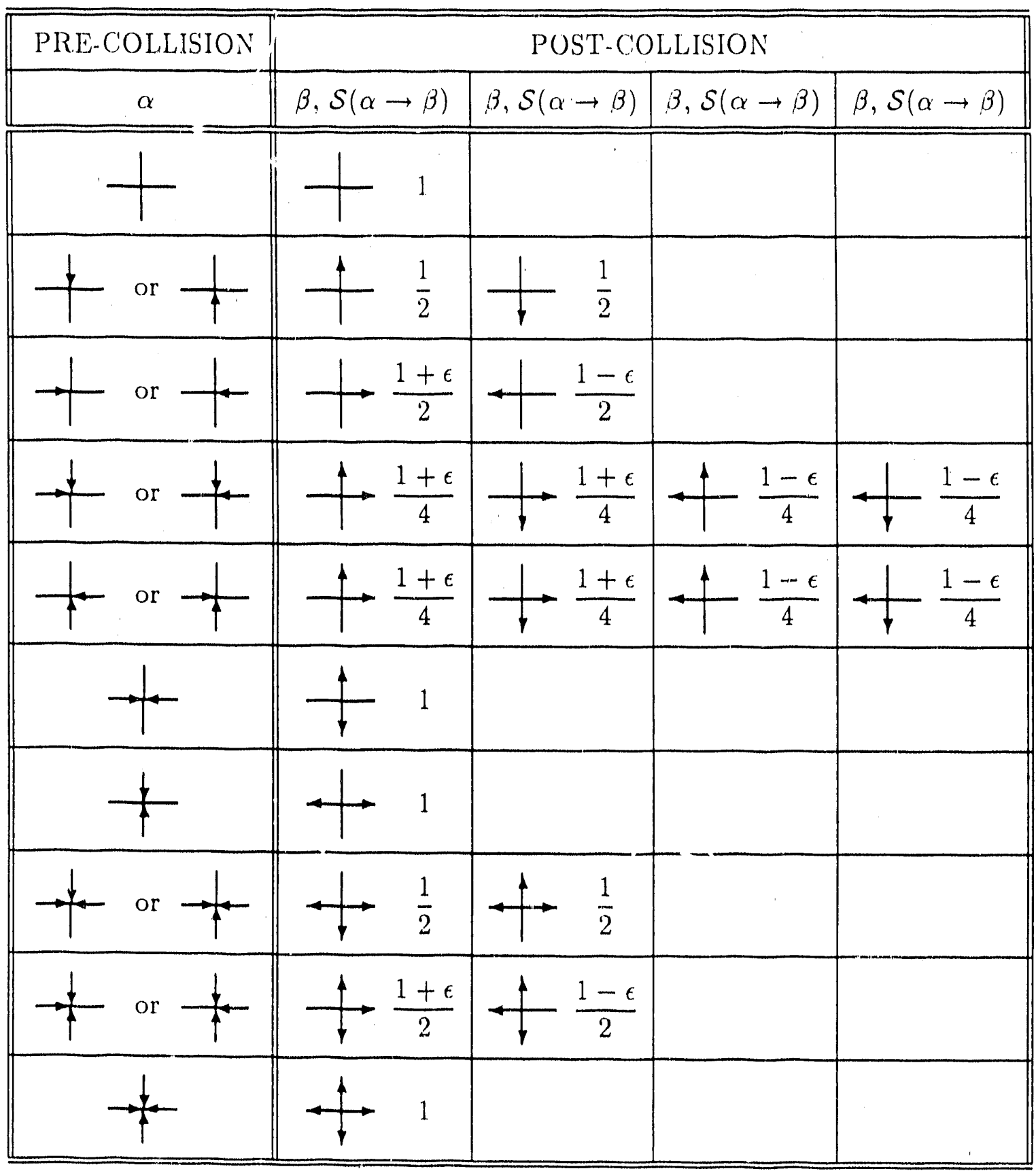


Table 3.8: Collision Rules for Two-Dimensional Advection-Diffusion. $a=(1+\epsilon) / 2$ and $\bar{a}=(1-\epsilon) / 2$.

\begin{tabular}{|c|c|c|c|c|c|c|c|c|c|}
\hline \multirow[t]{2}{*}{ Rule } & \multicolumn{4}{|c|}{$\alpha$} & \multicolumn{4}{|c|}{$\beta$} & \multirow[t]{2}{*}{$\mathcal{S}(\alpha \rightarrow \beta)$} \\
\hline & $\alpha_{0}$ & $\alpha_{1}$ & $\alpha_{2}$ & $\alpha_{3}$ & $\beta_{0}$ & $\beta_{1}$ & $\bar{\beta}_{2}$ & $\beta_{3}$ & \\
\hline$\overline{0}$ & 0 & 0 & 0 & 0 & 0 & $\overline{0}$ & $\overline{0}$ & 0 & $\overline{1}$ \\
\hline \multirow[t]{2}{*}{1} & 0 & 0 & 0 & 1 & 0 & $\overline{0}$ & 0 & 1 & $1 / 2$ \\
\hline & & & & & 0 & 1 & 0 & 0 & $1 / 2$ \\
\hline \multirow[t]{2}{*}{2} & 0 & 0 & 1 & 0 & 1 & 0 & 0 & & $a$ \\
\hline & & & & & 0 & 0 & 1 & 0 & $\bar{a}$ \\
\hline \multirow[t]{4}{*}{3} & 0 & 0 & 1 & 1 & 0 & 0 & 1 & 1 & $\bar{a} / 2$ \\
\hline & & & & & 1 & 0 & 0 & & $a / 2$ \\
\hline & & & & & 0 & 1 & 1 & & $\bar{a} / 2$ \\
\hline & & & & & 1 & 1 & 0 & 0 & $a / 2$ \\
\hline \multirow[t]{2}{*}{4} & 0 & 1 & 0 & 0 & 0 & 0 & 0 & & $1 / 2$ \\
\hline & & & & & 0 & 1 & 0 & 0 & $1 / 2$ \\
\hline 5 & 0 & 1 & 0 & 1 & 1 & 0 & 1 & 0 & 1 \\
\hline \multirow[t]{4}{*}{6} & 0 & 1 & 1 & $\overline{0}$ & 0 & 0 & 1 & & $\bar{a} / 2$ \\
\hline & & & & & 1 & 0 & 0 & 1 & $a / 2$ \\
\hline & & & & & 0 & 1 & 1 & 0 & $\bar{a} / 2$ \\
\hline & & & & & 1 & 1 & 0 & 0 & $a / 2$ \\
\hline \multirow[t]{2}{*}{7} & 0 & 1 & 1 & 1 & 0 & 1 & 1 & 1 & $\bar{a}$ \\
\hline & & & & & 1 & 1 & 0 & 1 & $a$ \\
\hline \multirow[t]{2}{*}{8} & 1 & 0 & 0 & 0 & 0 & 0 & 1 & 0 & $\bar{a}$ \\
\hline & & & & & 1 & 0 & 0 & 0 & $a$ \\
\hline \multirow[t]{4}{*}{9} & 1 & 0 & 0 & 1 & 0 & 0 & $\overline{1}$ & & $\overline{\bar{a} / 2}$ \\
\hline & & & & & 1 & 0 & $c$ & 1 & $a / 2$ \\
\hline & & & & & 0 & 1 & 1 & 0 & $\bar{a} / 2$ \\
\hline & & & & & 1 & 1 & 0 & 0 & $a / 2$ \\
\hline 10 & 1 & 0 & 1 & 0 & 0 & 1 & c & 1 & 1 \\
\hline \multirow[t]{2}{*}{11} & 1 & 0 & 1 & 1 & 1 & 0 & 1 & 1 & $1 / 2$ \\
\hline & & & & & 1 & 1 & 1 & 0 & $1 / 2$ \\
\hline \multirow[t]{4}{*}{12} & 1 & 1 & $\overline{0}$ & $\overline{0}$ & 0 & 0 & 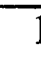 & & $\bar{a} / 2$ \\
\hline & & & & & 1 & 0 & c & & $a / 2$ \\
\hline & & & & & 0 & 1 & 1 & 0 & $\bar{a} / 2$ \\
\hline & & & & & 1 & 1 & I & $\underline{0}$ & $a / 2$ \\
\hline \multirow[t]{2}{*}{13} & 1 & 1 & 0 & 1 & 0 & 1 & 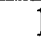 & & $\bar{a}$ \\
\hline & & & & & 1 & 1 & & 1 & $a$ \\
\hline \multirow[t]{2}{*}{14} & 1 & 1 & 1 & 0 & 1 & 0 & 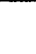 & & $1 / 2$ \\
\hline & & & & & 1 & 1 & & 0 & $1 / 2$ \\
\hline 15 & 1 & 1 & 1 & 1 & 1 & 1 & - & 1 & 1 \\
\hline
\end{tabular}




$$
\begin{aligned}
& +\bar{n}_{0} \hat{n}_{1} \bar{n}_{2} \hat{n}_{3}-i_{1} \bar{n}_{1} \hat{n}_{2} \bar{n}_{3} \\
& \mathcal{C}_{1}(\hat{\mathrm{n}})= \\
& +\frac{1}{2} \bar{n}_{0} \overline{n_{1}} \overline{\hat{n}}_{2} \hat{n}_{3}+\frac{1}{2} \bar{n}_{0} \overline{n_{1}} \hat{n}_{2} \hat{n}_{3}+\frac{1}{2} \hat{n}_{0} \overline{\hat{n}_{1}} \overline{\hat{n}_{2}} \hat{n}_{3}+\frac{1}{2} \hat{n}_{0} \overline{\hat{n}_{1}} \hat{n}_{2} \hat{n}_{3} \\
& -\frac{1}{2} \hat{n}_{0} \hat{n}_{1} \overline{\hat{n}_{2}} \overline{\hat{n}_{3}}-\frac{1}{2} \bar{n}_{0} \hat{n}_{1} \hat{n}_{2} \overline{\hat{n}_{3}}-\frac{1}{2} \hat{n}_{0} \hat{n}_{1} \overline{\hat{n}_{2}} \overline{\hat{n}_{3}}-\frac{1}{2} \hat{n}_{0} \hat{n}_{1} \hat{n}_{2} \overline{\hat{n}_{3}} \\
& +\hat{n}_{0} \overline{\hat{n}_{1}} \hat{n}_{2} \overline{\hat{n}_{3}}-\overline{\hat{n}_{0}} \hat{n}_{1} \overline{\hat{n}_{2}} \hat{n}_{3} \\
& \mathcal{C}_{2}(\hat{\mathbf{n}})= \\
& +\bar{a} \hat{n}_{0} \overline{\hat{n}_{1}} \overline{\hat{n}_{2}} \overline{\hat{n}_{3}}+\bar{a} \hat{n}_{0} \overline{\hat{n}_{1}} \overline{\hat{n}_{2}} \hat{n}_{3}+\bar{a} \hat{n}_{0} \hat{n}_{1} \overline{\hat{n}_{2}} \overline{\hat{n}_{3}}+\bar{a} \hat{n}_{0} \hat{n}_{1} \overline{\hat{n}_{2}} \hat{n}_{3} \\
& -a \overline{\hat{n}_{0}} \overline{\hat{n}_{1}} \hat{n}_{2} \overline{\hat{n}_{3}}-a \overline{\hat{n}_{0}} \overline{\hat{n}_{1}} \hat{n}_{2} \hat{n}_{3}-a \overline{\hat{n}_{0}} \hat{n}_{1} \hat{n}_{2} \overline{\hat{n}_{3}}-a \overline{\hat{n}_{0}} \hat{n}_{1} \hat{n}_{2} \hat{n}_{3} \\
& +\overline{\hat{n}_{0}} \hat{n}_{1} \overline{\hat{n}}_{2} \hat{n}_{3}-\hat{n}_{0} \overline{\hat{n}}_{1} \hat{n}_{2} \overline{\hat{n}_{3}} \\
& \mathcal{C}_{3}(\hat{\mathbf{n}})= \\
& +\frac{1}{2} \overline{\hat{n}_{0}} \hat{n}_{1} \overline{\hat{n}_{2}} \overline{\hat{n}_{3}}+\frac{1}{2} \bar{n}_{0} \hat{n}_{1} \hat{n}_{2} \overline{\hat{n}_{3}}+\frac{1}{2} \hat{n}_{0} \hat{n}_{1} \overline{\hat{n}_{2}} \overline{\hat{n}_{3}}+\frac{1}{2} \hat{n}_{0} \hat{n}_{1} \hat{n}_{2} \overline{\hat{n}_{3}} \\
& -\frac{1}{2} \overline{\hat{n}_{0}} \overline{\hat{n}_{1}} \overline{\hat{n}_{2}} \hat{n}_{3}-\frac{1}{2} \overline{\hat{n}_{0}} \overline{\hat{n}_{1}} \hat{n}_{2} \hat{n}_{3}-\frac{1}{2} \hat{n}_{0} \overline{\hat{n}_{1}} \overline{\hat{n}_{2}} \hat{n}_{3}-\frac{1}{2} \hat{n}_{0} \overline{\hat{n}_{1}} \hat{n}_{2} \hat{n}_{3} \\
& +\hat{n}_{0} \overline{\hat{n}_{1}} \hat{n}_{2} \overline{\hat{n}_{3}}-\overline{\hat{n}_{0}} \hat{n}_{1} \overline{\hat{n}}_{2} \hat{n}_{3}
\end{aligned}
$$

or, in vector notation,

$$
\begin{aligned}
& \mathcal{C}(\hat{\mathbf{n}})= \\
& \quad+\mathrm{q}_{1}\left[(1-2 a) \hat{n}_{0} \hat{n}_{2}+a \hat{n}_{2}-\bar{a} \hat{n}_{0}\right] \\
& \quad+\mathrm{q}_{2}\left[\hat{n}_{3}-\hat{n}_{1}\right] / 2 \\
& \quad+\mathrm{q}_{3}\left[\hat{n}_{0} \hat{n}_{2} \hat{n}_{3}+\hat{n}_{0} \hat{n}_{1} \hat{n}_{2}-\hat{n}_{1} \hat{n}_{2} \hat{n}_{3}-\hat{n}_{0} \hat{n}_{1} \hat{n}_{3}+\hat{n}_{1} \hat{n}_{3}-\hat{n}_{0} \hat{n}_{2}\right]
\end{aligned}
$$


where column vectors $\mathrm{q}_{k}$ are given by

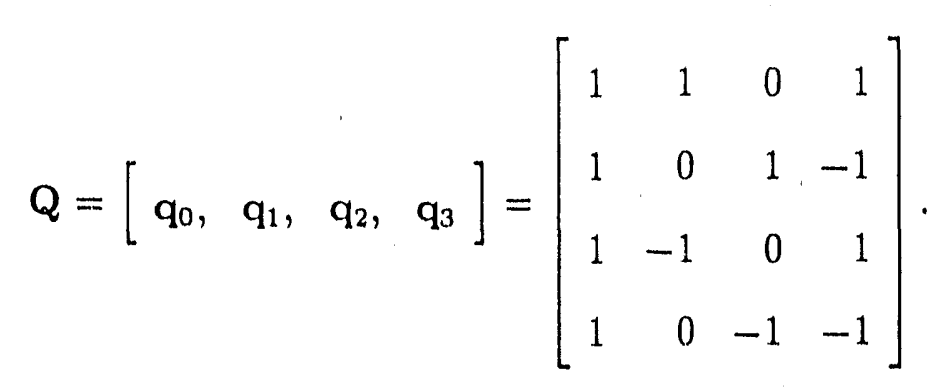

(We find later in the text that $\mathbf{Q}$ is the eigenmatrix of the linearized collision operator.)

\subsubsection{Lattice Boltzmann Approximation}

The Lattice Boltzmann Approximation is obtained by ensemble averaging Eq. 3.54 and neglecting the covariances. With $n_{k} \approx\left\langle\hat{n}_{k}\right\rangle$ the resulting approximation is

$$
\mathcal{C}(\mathbf{n})=\mathcal{C}^{(0)}(\mathbf{n})+\delta \mathcal{C}^{(1)}(\mathbf{n})
$$

in which

$$
\begin{aligned}
\mathcal{C}^{(0)}(\mathbf{n}) & =\mathrm{q}_{1}\left(n_{2}-n_{0}\right) / 2+\mathrm{q}_{2}\left(n_{3}-n_{1}\right) / 2 \\
& +\mathrm{q}_{3}\left(n_{0} n_{2} n_{3}+n_{0} n_{1} n_{2}-n_{1} n_{2} n_{3}-n_{0} n_{1} n_{3}+n_{1} n_{3}-n_{0} n_{2}\right), \\
\mathcal{C}^{(1)}(\mathbf{n}) & =\mathbf{q}_{1} K\left(n_{0}+n_{2}-2 n_{0} n_{2}\right) / 2
\end{aligned}
$$

where we have used the relations Eq. 3.52 and Eq. 3.53. Then

$$
\mathcal{A} \mathrm{n}=\mathrm{n}+\mathcal{C}(\mathrm{n})
$$

may be used as a lattice Boltzmann method with $\mathbf{n} \in[0,1]^{4}$. The remaining analysis derives the hydrodynamical equations of this method. 


\subsubsection{Equilibrium Analysis}

Let us use the direct method of Section 2.2.1 for determining the equilibrium. We will find one form of the equilibrium.

$$
\mathbf{n}^{(0)}=u \mathbf{q}_{0}
$$

To see this. first set the leading term of the collision operator to zero,

$$
\mathcal{C}^{(0)}(\mathrm{n})=0=\left[\begin{array}{l}
0 \\
0 \\
0 \\
0
\end{array}\right]
$$

This implies $\mathcal{C}_{0}^{(0)}(\mathbf{n})=\mathcal{C}_{2}^{(0)}(\mathbf{n})$, whict. forces $n_{0}=n_{2} \equiv v$. Similarly, $\mathcal{C}_{1}^{(0)}(\mathbf{n})=\mathcal{C}_{3}^{(0)}(\mathbf{n})$ implies $n_{1}=n_{3} \equiv w$. Substituting these back intc Eq. ?.57 we find that either

$$
v=w
$$

or

$$
w=\frac{v}{2 v-1} .
$$

This yields two forms for the equilibrium:

$$
\left[\begin{array}{l}
u \\
u \\
u \\
u
\end{array}\right] \quad(v=w \equiv u) \text {, and }\left[\begin{array}{c}
v \\
v /(2 v-1) \\
v \\
v /(2 v-1)
\end{array}\right] \quad(v=v /(2 v-1)) \text {. }
$$

Note that $f(?) \equiv v /(2 v-1) \notin(0,1)$ for $v \in(0,1)$. Thus, the second form does not apply; occupation numbers cannot lie outside $[0,1]$. We will use the first form for the 
remainder of the analysis. Then the equilibrium is

$$
\mathbf{n}^{(0)}=\left[\begin{array}{l}
u \\
u \\
u \\
u
\end{array}\right] .
$$

\subsubsection{Linearized Collision Operator}

Using the non-trivial equilibrium solution, $\mathrm{n}^{(0)}$, we can now linearize the leading order of the collision operator,

$$
\mathcal{L}=\left.\frac{\partial}{\partial \mathbf{n}} \mathcal{C}^{(0)}(\mathbf{n})\right|_{\mathbf{n}=\mathbf{n}(0)}=\left[\mathcal{L}_{k, l}\right],
$$

where

$$
\mathcal{L}_{k, l}=\left.\frac{\partial}{\partial n_{l}} \mathcal{C}_{k}^{(0)}(\mathbf{n})\right|_{\mathbf{n}=\mathbf{n}(0)} .
$$

Completing the calculation, we find

$$
\begin{gathered}
\left.\frac{\partial}{\partial n_{k}} \mathcal{C}_{k}^{(0)}(\mathbf{n})\right|_{\mathbf{n}=\mathbf{n}^{(0)}}=\left(2 u^{2}-2 u-1\right) / 2,\left.\quad \frac{\partial}{\partial n_{k+1}} \mathcal{C}_{k}^{(0)}(\mathbf{n})\right|_{\mathbf{n}=\mathbf{n}^{(0)}}=u(1-u), \\
\left.\frac{\partial}{\partial n_{k+2}} \mathcal{C}_{k}^{(0)}(\mathbf{n})\right|_{\mathbf{n}=\mathbf{n}^{(0)}}=\left(2 u^{2}-2 u+1\right) / 2,\left.\frac{\partial}{\partial n_{k+3}} \mathcal{C}_{k}^{(0)}(\mathbf{n})\right|_{\mathbf{n}=\mathbf{n}^{(0)}}=u(1-u),
\end{gathered}
$$

for $k \in\{0,1,2,3\}$ and all indices are evaluated modulo 4 . Then $\mathcal{L}$ may be written

$$
\mathcal{L}=\frac{1}{2}\left[\begin{array}{llll}
2 u^{2}-2 u-1 & 2 u(1-u) & 2 u^{2}-2 u+1 & 2 u(1-u) \\
2 u(1-u) & 2 u^{2}-2 u-1 & 2 u(1-u) & 2 u^{2}-2 u+i \\
2 u^{2}-2 u+1 & 2 u(1-u) & 2 u^{2}-2 u-1 & 2 u(1-\vdots) \\
2 u(1-u) & 2 u^{2}-2 u+1 & 2 u(1-u) & 2 u^{2}-2 u-1
\end{array}\right] .
$$




\subsubsection{Eigenvalues and Eigenvectors}

Note that indeed $\mathcal{L}$ is a symmetric circulant, so it has a complete set of orthogonal real eigenvectors with real eigenvalues. The eigenvalues of $\mathcal{L}$ are given by

$$
\text { eigenvalues }(\mathcal{L})=\left(\lambda_{0}, \lambda_{1}, \lambda_{2}, \lambda_{3}\right)=(0,-1,-1,-4 u(1-u))
$$

Indeed we see that $\mathcal{L}$ is nonpositive definite for $u \in[0,1]$.

As previously suggested Eq. 3.55 lists the (unnormalized) eigenvectors of $\mathcal{L}$, which one can find, e.g., by noting that $\mathcal{L}$ is a circulant and using the formulas for eigenvectors of circulants [3, pp. 242-3]. Note that the dimension of the nullspace of $\mathcal{L}$ is one, the nullspace being spanned by $q_{0}$, i.e.,

$$
\operatorname{nullspace}(\mathcal{L})=\operatorname{span}\left(\mathbf{q}_{0}\right)
$$

The pseudo-inverse of $\mathcal{L}$ may be written

$$
\mathcal{L}^{+}=\sum_{k \ni \lambda_{k} \neq 0} \frac{1}{\lambda_{k}} \frac{\mathbf{q}_{k} \mathbf{q}_{k}^{T}}{\mathbf{q}_{k} \cdot \mathbf{q}_{k}} .
$$

Noting that the eigenmatrices for this and the $\mathrm{LB}_{1}$ (and $\mathrm{LB}_{2}$ ) lattice Bolizmann methods are identical, Table 2.4 lists the componentwise eigenvector products for the lattice method of this section also.

\subsubsection{Discrete Chapman-Enskog Expansion}

We assume

$$
\begin{aligned}
\epsilon & =K \delta \\
\Delta x & =L \delta \\
\Delta y & =L \delta \\
\Delta t & =T \delta^{2},
\end{aligned}
$$


in which $K>0$, and $L$ and $T$ are the respective spatial and temporal scale lengths. Now, we may readily apply the discrete Chapman-Enskog analysis.

\section{Gradient Expressiors}

Note that in the present method the unit velocity vectors, $\vec{e}_{k}(k \in\{0,1,2,3\})$, are the same as in $L B_{1}$ and $L B_{2}$. Thus, gradient expressions involving the unit velocity vectors are expressed in the same fashion (see Eq. 2.25).

\section{Matching the Expansions}

Employing the results of Section 2.3.5, order by order we obtain the following hierarchy:

$\mathcal{O}[1]: \mathcal{C}^{(0)}\left(\mathbf{n}^{(0)}\right)=0$, as desired.

$\mathcal{O}[\delta]:$ We find

$$
\begin{aligned}
\mathcal{L} \cdot \mathbf{n}^{(1)} & =L\left[\vec{e}_{k} \cdot \nabla\right] \mathbf{n}^{(0)}-\mathcal{C}^{(1)}\left(\mathbf{n}^{(0)}\right) \\
& =\left(L u_{x}-K u(1-u)\right) \mathbf{q}_{1}+\left(L u_{y}\right) \mathbf{q}_{1} \\
& \equiv \mathbf{g}^{(1)}
\end{aligned}
$$

- Consistency: Note that already $\mathrm{g}^{(1)}$ is orthogonal to nullspace $(\mathcal{L})$, i.e., $\mathrm{q}_{0}$. $\mathrm{g}^{(1)}=0$, so that no consistency condition is introduced at this order.

- Solve for $\mathbf{n}^{(1)}$ : We find

$$
\begin{aligned}
\mathbf{n}^{(1)} & =\mathcal{L}^{+} \cdot \mathbf{g}^{(1)}+\sigma^{(1)} \mathbf{q}_{0} \\
& =\sigma^{(1)} \mathbf{q}_{0}+\left(K u(1-u)-L u_{x}\right) \mathbf{q}_{1}+\left(-L u_{y} ! \mathbf{q}_{2},\right.
\end{aligned}
$$


where $\sigma^{(1)}$ is the arbitrary parameter introduced by the nullspace of the linearized collision operator. One parameter is introduced since

$$
\operatorname{dim}(\operatorname{nuil} \operatorname{space}(\mathcal{L}))=1
$$

$\mathcal{O}\left[\delta^{2}\right]:$ We find

$$
\begin{aligned}
\dot{L} \cdot \mathbf{n}^{(2)}= & \frac{L^{2}}{2}\left[\left(\vec{e}_{k} \cdot \nabla\right)^{2}\right] \mathbf{n}^{(0)}+T \frac{\partial}{\partial t} \mathbf{n}^{(0)}+L\left[\vec{e}_{k} \cdot \nabla\right] \mathbf{n}^{(1)} \\
- & \frac{1}{2} \mathcal{D}^{2} \mathcal{C}^{(0)}(\mathbf{n}) \cdot \mathbf{n}^{(1)} \mathbf{n}^{(1)}-\mathcal{D} \mathcal{C}^{(1)}\left(\mathbf{n}^{(0)}\right) \cdot \mathbf{n}^{(1)} \\
= & \left(T u_{t}+\frac{K L}{2} \frac{\partial}{\partial x}(K u(1-u))-\frac{L^{2}}{4} \nabla^{2} u\right) \mathbf{q}_{0} \\
+ & \left(L \sigma_{x}^{(1)}+K(2 u-1) \sigma^{(1)}\right) \mathbf{q}_{1}+\left(L \sigma_{y}^{(1)}\right) \mathbf{q}_{2} \\
+ & \left(2 K L \frac{\partial}{\partial x} \cdot(K u(1-u))-\frac{L^{2}}{4}\left(u_{x x}-u_{y y}\right)\right. \\
& \left.(1-2 u)\left(\left(L u_{y}\right)^{2}-\left(K u(1-u)-L u_{x}\right)^{2}\right)\right) \mathbf{q}_{3} \\
\equiv & \mathbf{g}^{(2)}
\end{aligned}
$$

in which

$$
\begin{aligned}
& \mathcal{D}^{2} \mathcal{C}^{(0)}(\mathbf{n}) \cdot \mathbf{n}^{(1)} \mathbf{n}^{(1)}=2(2 u-1)\left(\left(L u_{y}\right)^{2}-\left(K u(1-u)-L u_{x}\right)^{2}\right) \mathbf{q}_{3} \\
& \mathcal{D C} \mathcal{C}^{(1)}\left(\mathbf{n}^{(0)}\right) \cdot \mathbf{n}^{(1)}=K \sigma^{(1)}(1-2 u) \mathbf{q}_{1} .
\end{aligned}
$$

- Consistency: Setting $\mathrm{q}_{0} \cdot \mathrm{g}^{(2)}=0$ we find the consistency condition,

$$
\frac{\partial}{\partial t} u+c \frac{\partial}{\partial x}(u(1-u))=\nu\left(\frac{\partial^{2}}{\partial x^{2}} u+\frac{\partial^{2}}{\partial y^{2}} u\right)
$$

where

$$
\begin{aligned}
& c=\frac{K L}{2 T}, \\
& \nu=\frac{L^{2}}{4 T} .
\end{aligned}
$$


Note that we can now write $\Delta t$ and $\epsilon$ in terms of $c, \nu, \Delta x$, and $\Delta y$ :

$$
\begin{aligned}
\epsilon & =\frac{c \Delta x}{2 \nu}, \\
\Delta t & =\frac{(\Delta x)^{2}}{4 \nu} .
\end{aligned}
$$

Then with the linear change of variable,

$$
\rho \equiv c(1-2 u)
$$

Eq. 3.58 becomes Eq. 3.51 .

- Solve for $\mathrm{n}^{(2)}$ : We have

$$
\begin{aligned}
\mathbf{n}^{(2)}= & \mathcal{L}^{+} \cdot \mathbf{g}^{(2)}+\sigma^{(2)} \mathbf{q}_{0} \\
= & \mathbf{q}_{0} \sigma^{(2)}+\mathbf{q}_{1}\left[K(1-2 u) \sigma^{(1)}-L \sigma_{x}^{(1)}\right]+\mathbf{q}_{2}\left[-\sigma_{y}^{(1)}\right] \\
& +\mathbf{q}_{3} \frac{1}{16 u(1-u)}\left[L^{2}\left(u_{x x}-u_{y y}\right)-K L \frac{\partial}{\partial x}(u(1-u))\right. \\
& \left.4(2 u-1)\left(\left(L u_{y}\right)^{2}-\left(K u(1-u)-L u_{x}\right)^{2}\right)\right] .
\end{aligned}
$$

where $\sigma^{(2)}$ is the arbitrary scalar parameter introduced by the nullspace of the linearized collision operator.

$\mathcal{O}\left[\delta^{3}\right]:$ We find

$$
\begin{aligned}
\mathcal{L} & \cdot \mathbf{n}^{(3)} \\
& =\left[\frac{L^{3}}{3 !}\left(\vec{e}_{k} \cdot \nabla\right)^{3}+L T\left(\vec{e}_{k} \cdot \nabla\right) \frac{\partial}{\partial t}\right] \mathbf{n}^{(0)}+\left[\frac{L^{2}}{2 !}\left(\vec{e}_{k} \cdot \nabla\right)^{2}+T \frac{\partial}{\partial t}\right] \mathbf{n}^{(1)} \\
& +\left[L\left(\vec{e}_{k} \cdot \nabla\right)\right] \mathbf{n}^{(2)}-\frac{1}{6} \mathcal{D}^{3} \mathcal{C}^{(0)}\left(\mathbf{n}^{(0)}\right) \cdot \mathbf{n}^{(1)} \mathbf{n}^{(1)} \mathbf{n}^{(1)}-\mathcal{D}^{2} \mathcal{C}^{(0)}\left(\mathbf{n}^{(0)}\right) \cdot \mathbf{n}^{(1)} \mathbf{n}^{(2)} \\
& -\frac{1}{2} \mathcal{D}^{2} \mathcal{C}^{(1)}\left(\mathbf{n}^{(0)}\right) \cdot \mathbf{n}^{(1)} \mathbf{n}^{(1)}-6 \mathcal{D} \mathcal{C}^{(1)}\left(\mathbf{n}^{(0)}\right) \cdot \mathbf{n}^{(2)} \\
& =\sum_{k=0}^{3} \tilde{c}_{k}^{(3)} \mathbf{q}_{k},
\end{aligned}
$$


where

$$
\dot{c}_{0}^{(3)}=2 K L \frac{\partial}{\partial x}\left((1-2 u) \sigma^{(1)}\right)-L^{2} \frac{\partial^{2}}{\partial x^{2}} \sigma^{(1)}+\frac{L^{2}}{2} \frac{\partial^{2}}{\partial x^{2}} \sigma^{(1)}+T^{\prime} \frac{\partial}{\partial t} \sigma^{(1)},
$$

and $c_{k}^{(3)}$ remains uncalculated for $k \in\{1,2,3\}$. Although not shown here, each of $\mathcal{D}^{3} \mathcal{C}^{(0)}\left(n^{(0)}\right) \cdot n^{(1)} n^{(1)} n^{(1)}, \mathcal{D}^{2} \mathcal{C}^{(0)}\left(n^{(0)}\right) \cdot n^{(1)} n^{(2)}, \mathcal{D}^{2} \mathcal{C}^{(1)}\left(n^{(0)}\right) \cdot n^{(1)} n^{(1)}$, and $\mathcal{D} \mathcal{C}^{(1)}\left(n^{(0)}\right)$. $\mathrm{n}^{(2)}$ are orthogonal to the nullspace of $\mathcal{L}$. They only contribute to the uncalculated coefficients $\tilde{c}_{k}^{(3)}, k \in\{1,2,3\}$ and do not affect the rlctermination of the consistency condition.

- Consistency: Setting $\mathbf{q}_{0} \cdot \mathbf{g}^{(3)}=0$ yields the consistency condition,

$$
\frac{\partial}{\partial t} \sigma^{(1)}+\frac{2 K L}{T} \frac{\partial}{\partial x}\left[(1-2 u) \sigma^{(1)}\right]=\frac{L^{2}}{2 T}\left(\frac{\partial^{2}}{\partial x^{2}} \sigma^{(1)}+\frac{\partial^{2}}{\partial y^{2}} \sigma^{(1)}\right)
$$

We have carried out the expansion this far to see that the $\mathcal{O}\left[\delta^{3}\right]$ consistency condition is satisfied with $\sigma^{(1)}(x, y ; t)=$ const. We would choose $\sigma^{(1)}(x, y ; t)=0$ were we to apply a formal consistency argument subsequent to the derivation. Further, we stop the expansion here since we are not interested in pursuing higher order consistency condition. An attempt at proving its convergence would reveal whether higher order consistency conditions would be necessary.

This complates the first application of the discrete Chapman-Enskog expansion. It determined that the hydrodynamical equation of the lattice Boltzmann method is Eq. 3.58, while the $\mathcal{O}\left[\delta^{2}\right]$ consistency condition is Eq. 3.59 .

\subsection{Conclusions}

This chapter presented three lattice methods and determined their hydrodynamical equations via the discrete Chapman-Enskog expansion. For two of the methods, 
through consistency and stability arguments, we proved second-order convergence in the $L_{1}$-norm. 


\section{Chapter 4}

\section{Computational Studies}

This chapter provides computational evidence supporting the theory developed in the previous two (hapters for the following lattice Boltzmann methods: $L_{1} B_{1}$ (of Sections 2.1-2.4), $\mathrm{LB}_{2}$ (of Section 3.1), the method for the one-dimensional viscous Burgers equation (of Section 3.2), and the method for a two-dimensional advection-diffusion equation (of Section 3.3). Section 4.1 examines the computational results for $L B_{1}$, Section 4.2 for $\mathrm{LB}_{2}$, Section 4.3 for the method for the one-dimensional viscous Burgers equation, and Section 4.4 for the method for two-dimensional advection-diffusion.

The numerical studies yield quantitative results regarding the order of convergence of the various lattice Boltzmann methods. Coarser grid solutions computed by the lattice Boltzmann methods are compared with fine grid solutions computed by finite difference methods (the finite difference-computed solutions are used in place of analytic solutions). All the finite difference methods are conservative monotone schemes. We find that the studies substantiate the convergence results reported for each lattice Boltzmann method, excepting the lattice Boltzmann method for a two-dimensional advection-diffusion equation ${ }^{1}$.

\footnotetext{
${ }^{1}$ No proof of convergence was given for that method; however, the computational results reported in Section 4.4 suggest second-order convergence.
} 
llo now develop some notion for compring the linite difference- and latetice Boltzmann-computed solutions. Consider solving the time-dependent partial differantial cepuation,

$$
u_{t}+F(u)=0
$$

with some appropriate initial and boundary conditions, where t'e functional $F$ depends on $u(\vec{x} ; t)$ and its derivatives. Then let $V_{i}^{n}$ and $r_{r}^{n}$ denote the finits difference and lattice Boltzmann approximations to $u(\vec{\imath} \Delta x ; n \Delta t)$, respectively, for grid size $N$, where $u(\vec{x} ; t)$ is an exact solution to the partial differential equation. ${ }^{2}$ Then let.

$$
E^{(N)}\left(\vec{\imath} ; r_{2}\right)=U_{r}^{n}-V_{r}^{n},
$$

with $E^{(N)}(n)$ being a vector of the $E^{(N)}(\vec{\imath} ; n)$ over the spatial grid points $\vec{\imath}^{3}$ Quantitative results are reported in the $L_{1^{-}}$and $L_{\infty}$-norms. In one dimension, consider an error vector $E$, then the norms are defined by

$$
\begin{aligned}
\|E\|_{\ell_{1}} & =\frac{\Delta x}{L} \sum_{i}\left|E_{i}\right|, \\
\|E\|_{\ell_{\infty}} & =\sup _{i}\left|E_{i}\right|,
\end{aligned}
$$

where $L$ is the spatial scale length. In two dimensions, consider a column vector $E$ then the norms are defined as follows:

$$
\begin{aligned}
\|E\|_{\ell_{1}} & =\frac{\Delta x \Delta y}{L^{(x)} L^{(y)}} \sum_{i, j}\left|E_{i, j}\right|, \\
\|E\|_{\ell_{\infty}} & =\sup _{i, j}\left|E_{i, j}\right|
\end{aligned}
$$

where $L^{(x)}$ and $L^{(y)}$ denote the $x$ and $y$ scale lengths, respectively. (We use $\Delta x=\Delta y$ and $L=L^{(x)}=L^{(y)}$ in the two-dimensional numerical studies.) We pay particular

\footnotetext{
"We will be using a finer grid to compute the finite difference solution than to compute the lattice Boltzmann solution. Then relative to the lattice Boltzmann-romputed solution, the finite difference-computed solution can be viewed as an exact solution.

"I 1 one dimension $\vec{\imath}=i$; in two, $\vec{\imath}=(i, j)$.
} 
attention to the ratios,

$$
\left\|E^{(N)}\right\|_{\ell_{1}} /\left\|E^{(2 N)}\right\|_{\ell_{1}} \text { and }\left\|E^{(N)}\right\|_{\ell_{\infty}} /\left\|E^{(2 N)}\right\|_{\ell_{\infty}} \text {, }
$$

both of which for $\mathcal{O}\left[\delta^{2}\right]$ convergence should be near four.

\section{$4.1 \quad \mathrm{LB}_{1}$}

This section presents numerical results regarding the order of convergence for lattice Boltzmann method, $\mathrm{LB}_{1}$. We find that they confirm (and suggest extension of) the theoretical results reported in Chapter 2 for this lattice Boltzmann method. ${ }^{4}$ Specifically, we introduce a finite difference method to generate reference solutions with which to compare the lattice Boltzmann computations. Then we compare the solutions computed by the two different methods and discuss the results.

\subsubsection{Finite Difference Method}

Consider the conservative finite difference method,

$$
\begin{aligned}
& U_{i, j}^{n+1}=U_{i, j}^{n} \\
& +\frac{\nu \Delta t}{\Delta x}\left[\frac{D\left(U_{i+1, j}^{n}\right)+D\left(U_{i, j}^{n}\right)}{2}\left(\frac{U_{i+1, j}^{n}-U_{i, j}^{n}}{\Delta x}\right)-\frac{D\left(U_{i, j}^{n}\right)+D\left(U_{i-1, j}^{n}\right)}{2}\left(\frac{U_{i, j}^{n}-U_{i-1, j}^{n}}{\Delta x}\right)\right] \\
& +\frac{\nu \Delta t}{\Delta y}\left[\frac{D\left(U_{i, j+1}^{n}\right)+D\left(U_{i, j}^{n}\right)}{2}\left(\frac{U_{i, j+1}^{n}-U_{i, j}^{n}}{\Delta y}\right)-\frac{D\left(U_{i, j}^{n}\right)+D\left(U_{i, j-1}^{n}\right)}{2}\left(\frac{U_{i, j}^{n}-U_{i, j-1}^{n}}{\Delta y}\right)\right],
\end{aligned}
$$

where $D(u)=\frac{1}{4 u(1-u)}-\frac{1}{2}$. With $\Delta x=\Delta y$, one can show that the method is an $\mathcal{O}\left[(\Delta x)^{2}\right]+\mathcal{O}[\Delta t]$ monotone finite difference method for $U_{i, j}^{n} \in(0,1)$ with a stability criterion of $\Delta t \leq(\Delta x)^{2} /(4 \nu)$, where $U_{i, j}^{n}$ approximates solutions to Eq. 2.29 .

\footnotetext{
${ }^{4} \mathrm{LB}_{1}$ is the running example of Chapter 2.
} 


\subsubsection{Numerical Results}

We compute solutions to $E_{1} .2 .29$ for $\left(x, y^{\prime \prime}\right) \in[0,1] \times[0,1], t>0$, periodic boundary conditions, and initial condition, $u(x, y ; 0)=u_{I}(x, y)=A \sin (2 \pi x) \sin (2 \pi y)+B$, for constants $A$ and $B$ such that $u_{I}(x, y) \in(0,1)$. We vary $A$ and $B$ to generate initial conditions for the lattice Boltzmann method within or not (wholly) within the domain of monotonicity, $\mathcal{E}=[(1-1 / \sqrt{5}) / 2,(1+1 / \sqrt{5}) / 2]^{4}$, as given in Lemma 2.53 .

While the lattice Boltzmann computations are on a grid of size $N \times N$, where $N<256$, the finite difference computations are computcd originally on a grid of $256 \times 256$ points and rendered on the coarser grids via pointwise projection ${ }^{5}$. To avoid conflict with the limit of stability in the finite difference computations, the associated time increment, $\Delta_{F D} t$, is half that limit, i.e., $\Delta_{F D} t=(\Delta x)^{2} /(8 \nu)$. For the cases in which $A=0.45, \Delta_{F D} t$ is a quarter of the stability limit. Note that the finite difference-computed solution retains its accuracy when projected onto the coarser grids.

We computed solutions for two differenct initial conditions. Figure 4.1 exhibits the solution at time $t=0$ and time $t=1 / 32$ for initial conditions in the domain of monotonicity for $L B_{1}$. Figure 4.2 exhibits the solution at time $t=0$ and time $t=1 / 32$ for initial conditions not everywhere satisfying the conditions of monotonicity for $L B_{1}$, e.g., the monotonicity conditions are not satisfied at the locations

$$
(x, y) \in\{(1 / 4,1 / 4),(3 / 4,1 / 4),(1 / 4,3 / 4),(3 / 4,3 / 4)\} .
$$

\footnotetext{
${ }^{5}$ Pointwise projection of the finite difference-computed solutions means that the approximation on a coarse grid is the value of the corresponding point on the finest grid. This is analogous to the pointwise projection operator, $P$, used in showing consistency of finite difference schemes, i.e., in one space dimension $P u(x, 1)=u(x, t)$, where $u(x, t)$ is an exact solution. A contrasting operator is the central averaging operator, $A u(x, t)=\int_{x-\Delta x}^{x+\Delta x} u(x, t) d x$.
} 
At these points the initial occupation numbers are outside the domain of motonicity. The graphs at $t=1 / 32$ are the result of the $256 \times 256$ finite difference computations.

\section{Quantitative Analysis}

The first case regards Tables 4.1 and 4.2. Here, the initial condition satisfies the conditions of monotonicity. We look at the ratio between the norm of the difference between the finite difference- and lattice Boltzmann-computed solutions for grid sizes a factor of two apart, expecting the tio to be near four. The $L_{1}$-norm results support the theoretical second-order convergence in that norm, and even suggest a slight superconvergence, i.e., convergence beyond the predicted $\mathcal{O}\left[\delta^{2}\right]$ rate. The $L_{\infty}$ norm results indicate that $L B_{1}$ may also be second-order convergent in the $L_{\infty}$-norm.

The second case regards Tables 4.3 and 4.4. Here, the initial condition does not everywhere satisfy the conditions of monotonicity for $\mathrm{LB}_{1}$. Again, we look at the ratio between the norm of difference between the finite difference- and lattice Boltzmann-computed solutions for grid sizes a factor of two apart, but not necessarily expecting the ratio to be four (because of a lack of a statement of convergence for initial conditions not satisfying the monotonicity conditions). The $L_{1}$-norm results show that the theoretical second-order convergence of $\mathrm{LB}_{1}$ may still hold for some initial conditions not everywhere satisfying the conditions of monotonicity. One might argue in favor of a slight superconvergence here, too. The $L_{\infty}$-norm results indicate similar findings, i.e., $L B_{1}$ may be second-order accurate in the $L_{\infty}$-norm for initial conditions not everywhere satisfying the conditions of monotonicity. 


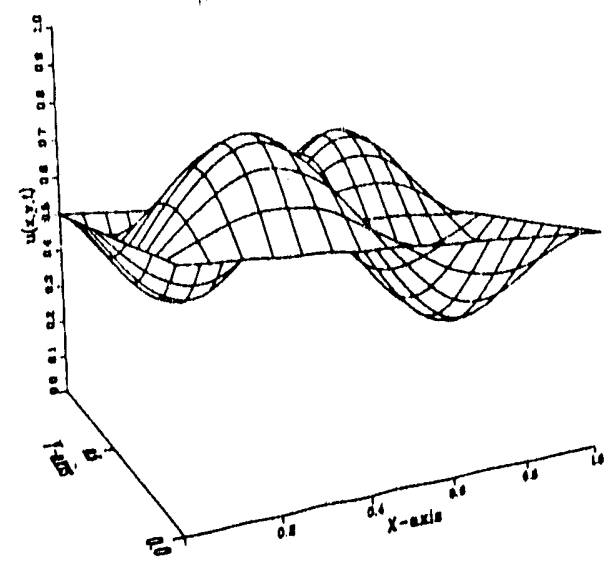

(a)

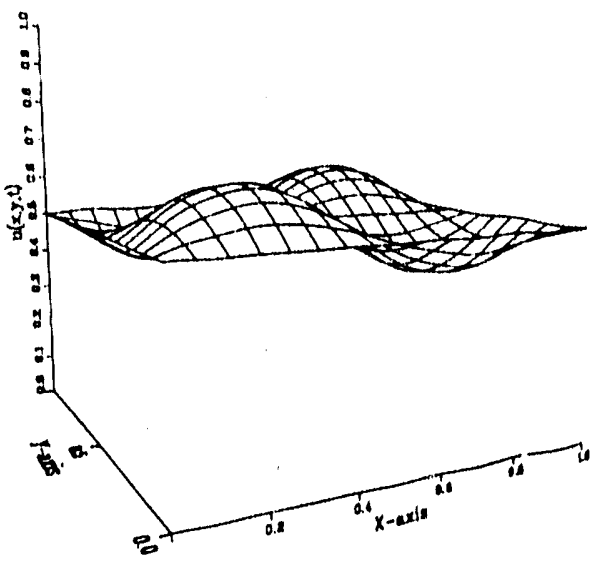

(b)

Figure 4.1: Evolution of $u(x, y ; t)$ according to Eq. 2.29 with $\nu=1 / 2$ : (a) Initial condition, $u(x, y ; 0)=u_{I}(x, y)=A \sin (2 \pi x) \sin (2 \pi y)+B$, where $A=1 / \sqrt{20}$ and $B=1 / 2$; (b) $u(x, y ; t)$ at $t=1 / 32$.

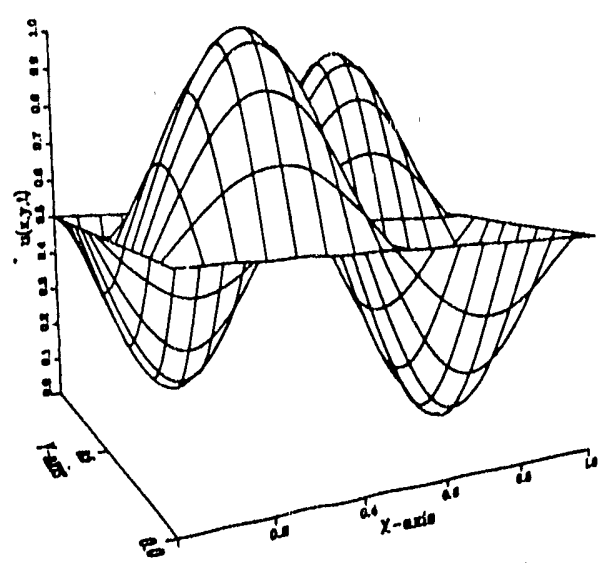

(a)

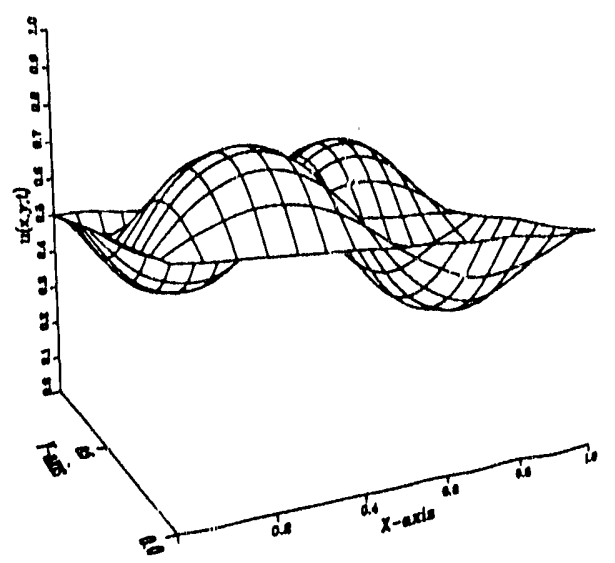

(b)

Figure 1.2: Evolution of $u(x, y ; t)$ according to Eq. 2.29 with $\nu=1 / 2$ : (a) Initial condition, $u(x, y ; 0)=u_{I}(x, y)=A \sin (2 \pi x) \sin (2 \pi y)+B$, where $A=0.45$ and $B=1 / 2$; (b) $u(x, y ; t)$ at $t=1 / 32$. 
Table 4.1: $L_{1}$-norm Comparison of $\mathrm{LB}_{1}$ - a'd Finite Difference-Computed Solutions with Initial Condition Parameters, $A=1 / \sqrt{20}$ and $B=1 / 2$.

\begin{tabular}{|c|c|c|c|c|c|c|}
\hline \multirow[b]{2}{*}{$N$} & \multirow[b]{2}{*}{$1 / N^{2}$} & \multicolumn{5}{|c|}{$\mid E^{(N)}$} \\
\hline & & $t=$ & $1 / 32$ & $1 / 16$ & $3 / 32$ & $1 / 8$ \\
\hline 8 & $1 / 64$ & & 0.00241 & 0.00301 & 0.00241 & 0.00170 \\
\hline 16 & $1 / 256$ & & 0.000605 & 0.000759 & 0.000624 & 0.000449 \\
\hline & $1 / 1024$ & & 0.000151 & 0.000190 & 0.000157 & 0.000114 \\
\hline 64 & $1 / 4096$ & & 0.0000366 & 0.0000465 & 0.0000387 & 0.0000279 \\
\hline 128 & $1 / 16384$ & & 0.00000804 & 0.0000107 & 0.00000902 & 0.00000662 \\
\hline$N$ & & & & $\|_{\ell_{1}} /$ & $\left.{ }^{N}\right) \|_{\ell_{1}}$ & \\
\hline 8 & & & 3.991 & 3.961 & 3.867 & 3.776 \\
\hline 16 & & & 4.010 & 4.002 & 3.987 & 3.955 \\
\hline$\overline{32}$ & & & 4.124 & $4.07 \overline{8}$ & 4.051 & 4.062 \\
\hline 64 & & & 4.550 & 4.336 & 4.287 & 4.223 \\
\hline
\end{tabular}

Table 4.2: $L_{\infty}$-norm Comparison of $\mathrm{LB}_{1}$ - and Finite Difference-Computed Solutions with Initial Condition Parameters, $A=1 / \sqrt{20}$ and $B=1 / 2$.

\begin{tabular}{||rr|llll||}
\hline \hline & \multicolumn{5}{|c||}{$\| E^{(N))^{\prime \prime}}$} \\
$N$ & $1 / N^{2}$ & $t=1 / 32$ & $1 / 16$ & $3 / 32$ & $1 / 8$ \\
\hline \hline 8 & $1 / 64$ & 0.0110 & 0.00885 & 0.00683 & 0.00476 \\
\hline 16 & $1 / 256$ & 0.00191 & 0.00194 & 0.00157 & 0.00113 \\
\hline 32 & $1 / 1024$ & 0.000455 & 0.000473 & 0.000387 & 0.000280 \\
\hline 64 & $1 / 4096$ & 0.000110 & 0.000115 & 0.0000950 & 0.0000675 \\
\hline 128 & $1 / 16384$ & 0.0000250 & 0.0000275 & 0.0000225 & 0.0000175 \\
\hline \hline$N$ & \multicolumn{5}{|c||}{$\left\|E^{(N)}\right\|_{\ell_{\infty}} /\left\|E^{(2 N)}\right\|_{\ell_{\infty}}$} \\
\hline 8 & & 5.755 & 4.572 & 4.343 & 4.192 \\
\hline 16 & 4.198 & 4.095 & 4.058 & 4.053 \\
\hline 32 & 4.137 & 4.108 & 4.078 & 4.147 \\
\hline 64 & 4.394 & 4.178 & 4.217 & 3.854 \\
\hline \hline
\end{tabular}


Table 4.3: $L_{1}$-norm Comparison of $L B_{1}$ - and Finite Difference-Computed Sorutions with Initial Condition Parameters, $A=0.45$ and $B=1 / 2$.

\begin{tabular}{||rr|llll||}
\hline \hline & & \multicolumn{5}{|c||}{$\left\|E^{(N)}\right\|_{\ell_{1}}$} \\
$N$ & $1 / N^{2}$ & $t=1 / 32$ & $1 / 16$ & $3 / 32$ & $1 / 8$ \\
\hline \hline 8 & $1 / 64$ & 0.00576 & 0.00427 & 0.00386 & 0.00281 \\
\hline 16 & $1 / 256$ & 0.00118 & 0.00125 & 0.00108 & 0.000789 \\
\hline 32 & $1 / 1024$ & 0.000251 & 0.000325 & 0.000279 & 0.000203 \\
\hline 64 & $1 / 4096$ & 0.0000603 & 0.0000818 & 0.0000704 & 0.0000513 \\
\hline 128 & $1 / 16384$ & 0.0000142 & 0.0000199 & 0.0000175 & 0.0000130 \\
\hline$N$ & & & $\left\|E^{(N)}\right\|_{\ell_{1}} /\left\|E^{(2 N)}\right\|_{\ell_{1}}$ & \\
\hline 8 & & 4.875 & 3.409 & 3.557 & 3.560 \\
\hline 16 & & 4.715 & 3.846 & 3.884 & 3.884 \\
\hline 32 & & 4.161 & 3.981 & 3.964 & 3.959 \\
\hline 64 & & 4.231 & 4.116 & 4.025 & 3.943 \\
\hline \hline
\end{tabular}

Table 4.4: $L_{\infty}$-norm Comparison of $\mathrm{LB}_{1}$ - and Finite Difference-Computed Solutions with Initial Condition Parameters, $A=0.45$ alıd $B=1 / 2$.

\begin{tabular}{||rr|llll||}
\hline \hline & & \multicolumn{5}{|c||}{$\left\|E^{(N)}\right\|_{\ell_{\infty}}$} \\
$N$ & $1 / N^{2}$ & $t=1 / 32$ & $1 / 16$ & $3 / 32$ & $1 / 8$ \\
\hline \hline 8 & $1 / 64$ & 0.0438 & 0.0173 & 0.0123 & 0.00854 \\
\hline 16 & $1 / 256$ & 0.00545 & 0.00344 & 0.00272 & 0.00199 \\
\hline 32 & $1 / 1024$ & 0.00124 & 0.000860 & 0.000685 & 0.000502 \\
\hline 65 & $1 / 4096$ & 0.000300 & 0.0002125 & 0.000172 & 0.000127 \\
\hline 128 & $1 / 16384$ & 0.0000700 & 0.0000525 & 0.0000425 & 0.0000325 \\
\hline \hline$N$ & \multicolumn{5}{|c|}{$\left\|E^{(N)}\right\|_{\ell_{\infty}} /\left\|E^{(2 N)}\right\|_{\ell_{\infty}}$} \\
\hline 8 & & 8.041 & 5.043 & 4.505 & 4.294 \\
\hline 16 & & 4.402 & 3.997 & 3.971 & 3.955 \\
\hline 32 & & 4.125 & 4.047 & 3.971 & 3.941 \\
\hline 64 & & 4.284 & 4.047 & 4.059 & 3.921 \\
\hline \hline
\end{tabular}




\section{$4.2 \quad \mathrm{LB}_{2}$}

This section presents numerical results regarding the order of convergence for lattice Boltzmann method, $\mathrm{LB}_{2}$. We find that they confirm the theoretical results reported in Section 3.1 for this lattice Boltzmann method. Specifically, we introduce a finite difference method to generate reference solutions with which to compare the lattice Boltzmann computations. Then we compare the solutions computed by the two different methods and discuss the results.

\subsubsection{Finite Difference Method}

Consider the conservative finite difference method, Eq. 4.1, in which

$$
D(u)=\frac{1}{2(1+u)(1-u)}-\frac{1}{2} .
$$

With $\Delta x=\Delta y$, one can show that the method is an $\mathcal{O}\left[(\Delta x)^{2}\right]+\mathcal{O}[\Delta t]$ monotone finite difference method for $U_{i, j}^{n} \in(0,1)$ with a stability criterion of $\Delta t \leq(\Delta x)^{2} /(4 \nu)$, where $U_{i, j}^{n}$ approximates solutions to Eq. 3.6.

\subsubsection{Numerical Results}

We compute solutions to Eq. 3.6 for $(x, y) \in[0,1] \times[0,1], t>0$, periodic boundary conditions, and initial condition, $u(x, y ; 0)=u_{I}(x, y)=A \sin (2 \pi x) \sin (2 \pi y)+B$, for constants $A$ and $B$ such that $u_{I}(x, y) \in(0,1)$. We vary $A$ and $B$ to generaic initial conditions for the lattice Boltzmann method within or not wholly within the domair. of monotonicity, $\mathcal{E}=[2 / 3,5 / 6]^{4}$, as given in Lemma 3.2 .

While the lattice Boltzmann computations are on the grid of size $N$, where $N<$ 256 , the finite difference computations are computed originally on a grid of $256 \times 256$ 
points and rendered onto the coarser grids via pointwise projection. To avoid conflict with the limit of stability in the finte difference computations, the associated time increment, $\Delta_{F D} t$, is half the stability limit. i.c., $\Delta_{F D} t=(\Delta x)^{2} /(8 \nu)$. For the cases in which $A=0.45, \Delta_{F D} t$ is a quarter of the stability limit. Note that the finite difference-computed solution retains its accuracy when projected onto the coarser grids.

We computed solutions for two differenct initial conditions. Figure 4.3 exhibits the solution at time $t=0$ and time $t=1 / 32$ for initial conditions satisfying the conditions of monotonicity for $\mathrm{LB}_{2}$. Figure 4.4 exhibits the solution at time $t=0$ and time $t=1 / 32$ for initial conditions not everywhere satisfying the conditions of monotonicity for $\mathrm{LB}_{2}$, e.g., the monotonicity conditions are not satisfied at the locations

$$
(x, y) \in\{(1 / 4,1 / 4),(3 / 4,1 / 4),(1 / 4,3 / 4),(3 / 4,3 / 4)\}
$$

At these points the initial occupation numbers are outside the domain of motonicity. The graphs at $t=1 / 32$ are the result of the $256 \times 256$ finite difference computations.

\section{Quantitative Analysis}

The first case regards Tables 4.5 and 4.6. Here, the initial condition satisfies the conditions of monotonicity for $\mathrm{LB}_{2}$. We look at the ratio between the norm of the difference between the finite difference- and lattice Boltzmann-computed solutions for grid sizes a factor of two apart, expecting the ratio to be near four. The $L_{1}$ norm results support the theoretical second order convergence of $\mathrm{LB}_{2}$, though not as strongly as in the corresponding (i.e., in the sense of both initial conditions satisfying the conditions of monotonicity) $L_{1} B_{1}$ case. The $L_{\infty}$-norm results suggest that $L_{1} B_{2}$ is 
second-order convergent in that norm, and, again, not as strongly as in the $\mathrm{LB}_{1}$ case.

The second case regards Tables 4.7 and 4.8. Here, the initial condition does not everywhere satisfy the conditions of monotonicity for $\mathrm{LB}_{2}$. Again, we look at the ratio between the norm of difference between the finite difference- and lattice Boltzmann-computed solutions for grid sizes a factor of two apart, but not necessarily expecting the ratio to be near four. The $L_{1}$-norm results show that the theoretical $L_{1}$-norm second-order convergence of $\mathrm{LB}_{2}$ indeed has trouble for initial conditions not everywhere satisfying the monotonicity conditions. (Of particular interest in this regard are the $N=64$ entries in Tables 4.7 and 4.8.) It should be noted that $N=128$ entries do uphold the ratio of sixteen for errors on grid sizes a factor of four apart, e.g., at $t=1 / 32$ the ratio, $\left\|E^{(16)}\right\|_{\ell_{1}} /\left\|E^{(128)}\right\|_{\ell_{1}}=16.042$ based on Table 4.7. The apparent discrepancy may be restricted to smaller grid sizes. 


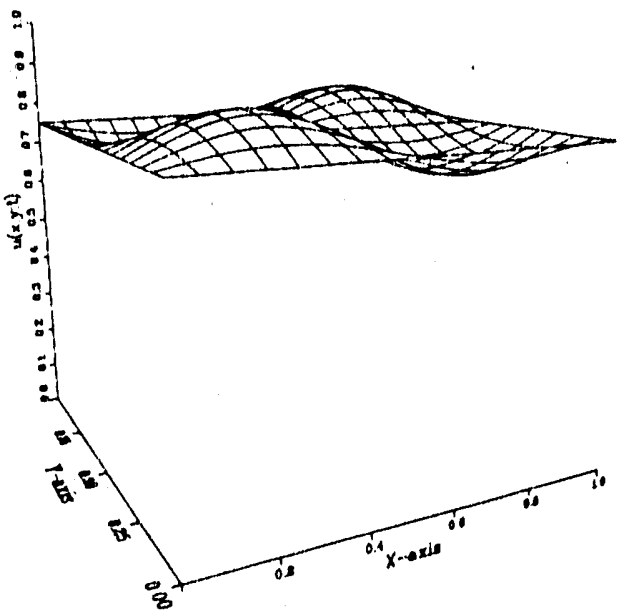

(a)

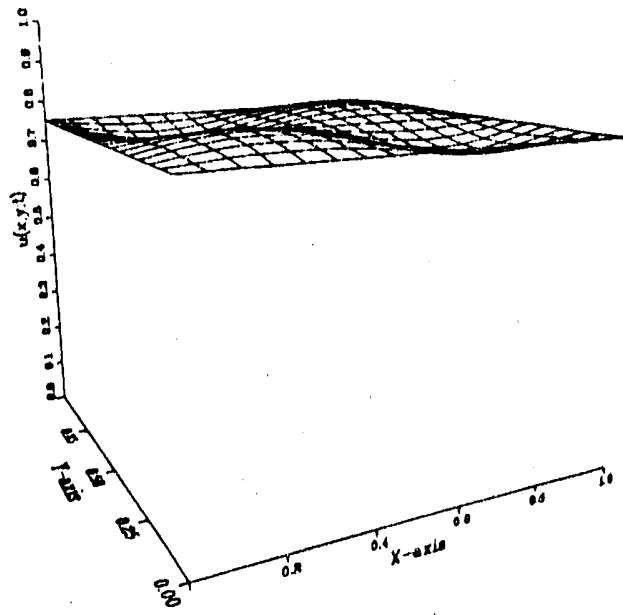

(b)

Figure 4.3: Evolution of $u(x, y ; t)$ according to Eq. 3.6 with $\nu=1 / 2:$ (a) Initial condition, $u(x, y ; 0)=u_{I}(x, y)=A \sin (2 \pi x) \sin (2 \pi y)+B$, where $A=1 / 12$ and $B=3 / 4 ;$ (b) $u(x, y ; t)$ at $t=1 / 32$.

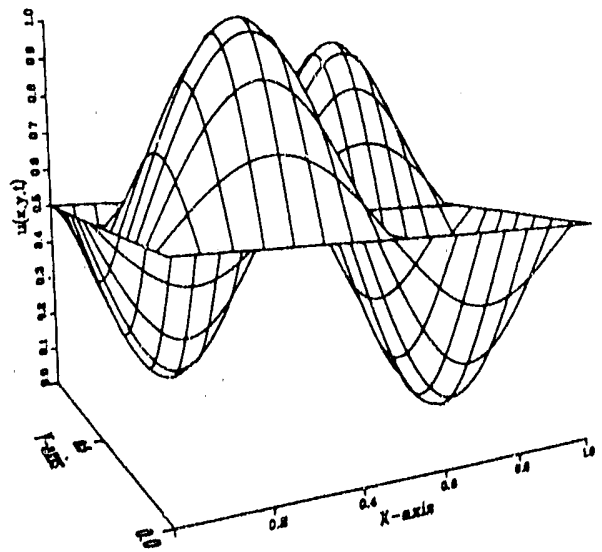

(a)

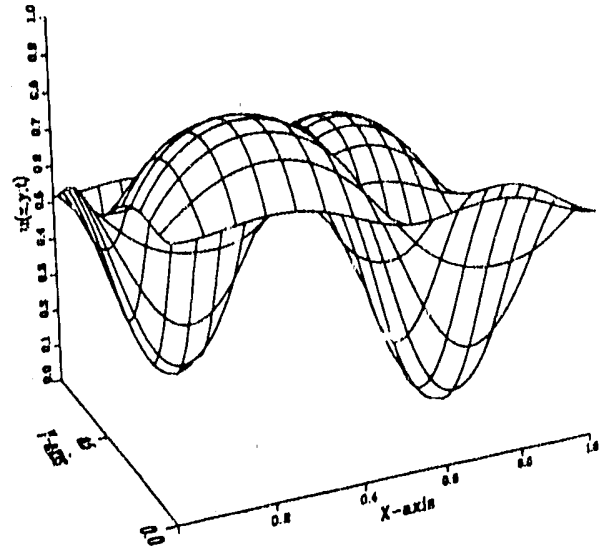

(b)

Figure 4.4: Evolution of $u(x, y ; t)$ according to Eq. 3.6 with $\nu=1 / 2$ : (a) Initial condition, $u(x, y ; 0)=u_{I}(x, y)=A \sin (2 \pi x) \sin (2 \pi y)+B$, where $A=0.45$ and $B=1 / 2$; (b) $u(x, y ; t)$ at $t=1 / 32$. 
Table 4.5: $L_{1}$-norm Comparison of $\mathrm{LB}_{2}$ - and Finite Difference-Computed Solutions with Initial Condition Parameters, $A=1 / 12$ and $B=3 / 4$.

\begin{tabular}{|c|c|c|c|c|c|c|}
\hline \multirow[b]{2}{*}{$N$} & \multirow[b]{2}{*}{$1 / N^{2}$} & \multicolumn{5}{|c|}{$E^{(N)}$} \\
\hline & & $t=$ & $1 / 32$ & $1 / 16$ & $3 / 32$ & $1 / 8$ \\
\hline$\overline{8}$ & $\overline{1 / 64}$ & & 0.000692 & 0.00202 & 0.00144 & 0.000827 \\
\hline 16 & $1 / 256$ & & 0.000276 & 0.000443 & 0.000321 & 0.000198 \\
\hline 32 & $1 / 1024$ & & 0.0000722 & 0.000106 & 0.0000782 & 0.0000491 \\
\hline 64 & $1 / 4096$ & & 0.0000180 & 0.0000264 & 0.0000197 & 0.0000123 \\
\hline 128 & $1 / 16384$ & & 0.00000463 & 0.00000668 & 0.00000502 & 0.00000316 \\
\hline$N$ & & & & $\left\|E^{(N)}\right\|_{\ell_{1}} / \|$ & N) $\|_{\ell_{1}}$ & \\
\hline$\overline{8}$ & & & 2.504 & 4.572 & 4.480 & 4.186 \\
\hline 16 & & & 3.827 & 4.166 & 4.106 & 4.021 \\
\hline 32 & & & 4.009 & 4.028 & 3.971 & 3.995 \\
\hline 64 & & & 3.893 & 3.948 & 3.924 & 3.893 \\
\hline
\end{tabular}

Table 4.6: $L_{\infty}$-norm Comparison of $\mathrm{LB}_{2}$ - and Finite Difference-Computed Solutions with Initial Condition Parameters, $A=1 / 12$ and $B=3 / 4$.

\begin{tabular}{|c|c|c|c|c|c|c|}
\hline \multirow[b]{2}{*}{$N$} & \multirow[b]{2}{*}{$1 / N^{2}$} & \multicolumn{5}{|c|}{$E$} \\
\hline & & $t=$ & $1 / 32$ & $1 / 16$ & ${ }^{\infty} 3 / 32$ & $1 / 8$ \\
\hline$\overline{8}$ & $1 / 64$ & & 0.00287 & $\overline{0.00504}$ & 0.00389 & $\overline{0.00226}$ \\
\hline 16 & $1 / 256$ & & 0.00104 & 0.00118 & 0.000830 & 0.000502 \\
\hline 32 & $1 / 1024$ & & 0.000263 & 0.000284 & 0.000200 & 0.000123 \\
\hline 64 & $1 / 4096$ & & 0.0000650 & 0.0000700 & 0.0000500 & 0.0000300 \\
\hline 128 & $1 / 16384$ & & 0.0000175 & 0.0000175 & 0.0000125 & 0.0000100 \\
\hline \multicolumn{2}{|c|}{$N$} & \multicolumn{5}{|c|}{$E^{(N)}\left\|_{\ell_{\infty}} /\right\| E^{(2 N)} \|_{\ell_{\infty}}$} \\
\hline 8 & & & 2.762 & 4.271 & 4.684 & 4.503 \\
\hline 16 & & & 3.952 & 4.141 & 4.151 & 4.100 \\
\hline 32 & & & 4.037 & 4.069 & 3.999 & 4.079 \\
\hline 64 & & & 3.711 & 3.997 & 3.995 & 3.000 \\
\hline
\end{tabular}


Table 4.7: $L_{1}$-norm Comparison of $L B_{2-}$ and Finito Difference-Computed Solutions with Initial Condition Parameters, $A=0.45$ and $B=1 / 2$.

\begin{tabular}{||cc|cccc||}
\hline \hline & & \multicolumn{5}{|c||}{$\left\|E^{(N)}\right\|_{i_{1}}$} \\
\hline$N$ & $1 / N^{2}$ & $t=1 / 32$ & $1 / 16$ & $3 / 32$ & $1 / 8$ \\
\hline \hline 8 & $1 / 64$ & 0.0191 & 0.0131 & 0.0126 & 0.0137 \\
\hline 16 & $1 / 256$ & 0.00483 & 0.00337 & 0.00368 & 0.00259 \\
\hline 32 & $1 / 1024$ & 0.00116 & 0.000914 & 0.000738 & 0.000594 \\
\hline 64 & $1 / 4096$ & 0.00617 & 0.00830 & 0.00920 & 0.00969 \\
\hline 128 & $1 / 16384$ & 0.0000657 & 0.0000471 & 0.0000359 & 0.0000295 \\
\hline$N$ & & & $\left\|E^{(N)}\right\|_{\ell_{1}} /\left\|E^{(2 N)}\right\|_{\ell_{1}}$ & \\
\hline 8 & & 3.949 & 3.884 & 3.426 & 5.296 \\
\hline 16 & & 4.158 & 3.684 & 4.985 & 4.366 \\
\hline 32 & 0.188 & 0.110 & 0.0803 & 0.0613 \\
\hline 64 & & 93.855 & 176.265 & 256.275 & 328.987 \\
\hline \hline
\end{tabular}

Table 4.8: $L_{\infty}$-norm Comparison of $\mathrm{LB}_{2-}$ and Finite Difference-Computed Solutions with Initial Condition Parameters, $A=0.45$ and $B=1 / 2$.

\begin{tabular}{|c|c|c|c|c|c|c|}
\hline \multirow[b]{2}{*}{$N$} & \multirow[b]{2}{*}{$1 / N^{2}$} & \multicolumn{5}{|c|}{$\left\|E^{(N)}\right\|$} \\
\hline & & $t=$ & $1 / 32$ & $1 / 16$ & $3 / 32$ & $1 / 8$ \\
\hline 8 & $1 / 64$ & & 0.0561 & 0.0859 & 0.114 & 0.148 \\
\hline 16 & $1 / 256$ & & 0.0141 & 0.0228 & 0.0320 & 0.0332 \\
\hline 32 & $1 / 1024$ & & 0.00462 & 0.00598 & 0.00946 & 0.0131 \\
\hline 64 & $1 / 4096$ & & 0.0130 & 0.0187 & 0.0262 & 0.0362 \\
\hline \multirow[t]{2}{*}{128} & $1 / 16384$ & & 0.000288 & 0.000338 & 0.000503 & 0.000777 \\
\hline & & \multicolumn{5}{|c|}{$E^{(N)}\left\|_{\ell_{\infty}} /\right\| E^{(2 N)} \|_{\ell_{\infty}}$} \\
\hline 8 & & & 3.984 & 3.765 & 3.555 & 4.440 \\
\hline 16 & & & 3.046 & 3.817 & 3.387 & 2.531 \\
\hline 32 & & & 0.355 & 0.319 & 0.361 & 0.362 \\
\hline 64 & & & 45.217 & 55.397 & 52.076 & 46.605 \\
\hline
\end{tabular}




\subsection{1-D Burgers' Equation LB Method.}

This section presents qualitative and quantitative numerical results regarding the order of convergence for lattice Boltzmann method for the one-dimensional viscous Burgers equation, the method as presented and discussed in Section 3.2. We find that the numerical results substantiate the theoretical second-order convergence of the method. Specifically, we give a finite difference method to generate reference solutions with which to compare the lattice Boltzmann computations. Then we compare the solutions computed by the two different methods and discuss the results.

\subsubsection{Finite Difference Method}

To generate accurate solutions to Eq. 3.34, with which to compare those computed by the lattice Boltzmann method, we use the finite difference method of Appendix C, i.e.,

$$
P_{i}^{n+1}=P_{i}^{n}-\frac{\Delta t}{4 \Delta x}\left[\left(P_{i+1}^{n}\right)^{2}-\left(P_{i-1}^{n}\right)^{2}\right]+\frac{\nu \Delta t}{(\Delta x)^{2}}\left[P_{i+1}^{n}-2 P_{i}^{n}+P_{i-1}^{n}\right]
$$

(cf. Eqs. C.3 and C.4 in which $U_{i}^{n} \equiv P_{i}^{n}$ ). One can show (see Appendix C) that this is an $\mathcal{O}\left[(\Delta x)^{2}\right]+\mathcal{O}[\Delta t]$ conservative monotone finite difference method for solutions $\rho(x ; t)$ of the one-dimensional viscous Burgers equation, $\rho_{t}+\rho \rho_{x}=\nu \rho_{x x}$, the solutions of which can be transformed to Eq. 3.34 with the substitution $\rho=c(1-2 u)$, where constant $c \geq\|\rho(x, 0)\|_{\ell_{\infty}}$. The stability criterion is that $\Delta t \leq(\Delta x)^{2} /(2 \nu)$. The difference method, Eq. 4.2, can be compared to the lattice Boltzmann method through the transformation, $P_{i}^{n}=c\left(1-2 U_{i}^{n}\right)$, where $U_{i}^{n}$ approximates solutions to Eq. 3.34 . 


\subsubsection{Numerical Results}

I'e compute solutions to Ey. 33.3.t for $x \in(0,1], 1>0$, periodic: boumdary conditions, and intial condition, $u(x ; 0)=u l(x ; 0)=(\cos (2 \pi, x)+1) / 2$. Figure d, 5 exhibits the initial condition and its evolution to time $t=1 / 4$ for $\nu=2^{-4}$.

\section{Qualitative Analysis}

Figure de exhibits a comparison of the finite difference- and lattice Boltamanncomputed solutions, $Y(x ; t)$ and $l(x ; t)$, respectively, at $t=1 / 4$ for $\nu=2^{-8}$. The linite difference-computed solution is on a grid of size $N=32768$; the lattice Boltzmann-computed solutions are on grids of size $N \in\{256,192,160,128\}$. 'The figure illustrates qualitative differences from the discrete Chapman-Enskog predicted behavior of the lattice Boltzmann method. Recall from Section 3.2 that the advection bias, $c=c \Delta x /(2 \nu)$. For the grid sizes $N \in\{256,192,160,128\}, \epsilon \in\{1 / 2,2 / 3,4 / 5,1\}$, respectively (for $c=1, \nu=2^{-8}$, and $\Delta x=1 / N$ ). For decreasing grid sizes $N$, the assumption that the advection bias is $\mathcal{O}[\Delta x]$ weakens, and tho hydrodynamical equations revealed in the discrete Chapman-Enskog procedure grow less accurate.

\section{Quantitative Analysis}

Table 4.9 regards the $L_{1}$ - and $L_{\infty}$-norm differences between the finite difference and lattice Boltzmann calculations at time $t=1 / 4$ for $\nu=2^{-8}$. The finite difference calculations are on a grid of size $N=32768$. Varying in the table is the grid size

for the lattice Boltzrnann method. Concentrating on the ratio $\left\|E^{(N)}\right\|\left\|_{\ell_{1}} /\right\| E^{(2 N)} \|_{\ell_{1}}$, the $L_{1}$-norm results in the table strongly support the theoretical $\mathcal{O}\left[\delta^{2}\right]$ convergence of the lattice Boltzmann method and its convergence in the $L_{1}$-norm. The $L_{\infty}$-norm 
Table 4.9: Comparison of Lattice Boltzmann- and Finite Difference-Computed Solutions to $\mathrm{Eq} .3 .34$ at $t=1 / 4$ with $\nu=2^{-8}$ and Initial Condition $u_{I}(x)=$ $(\cos (2 \pi x)+1) / 2$.

\begin{tabular}{||r||c|c||c|c||}
\hline \hline$N$ & $\left\|E^{(N)}\right\|_{\ell_{1}}$ & $\left\|E^{(N)}\right\|_{\ell_{1}} /\left\|E^{(2 N)}\right\|_{\ell_{1}}$ & $\left\|E^{(N)}\right\|_{\ell_{\infty}}$ & $\left\|E^{(N)}\right\|_{l_{\infty}}\left\|E^{(2 N)}\right\|_{l_{\infty}}$ \\
\hline \hline 256 & $1.042 \times 10^{-2}$ & 4.436 & $1.741 \times 10^{-2}$ & 4.455 \\
\hline 512 & $2.349 \times 10^{-3}$ & 4.099 & $3.908 \times 10^{\cdots 3}$ & 4.096 \\
\hline 1024 & $5.731 \times 10^{-4}$ & 4.026 & $9.540 \times 10^{-4}$ & 4.025 \\
\hline 2048 & $1.423 \times 10^{-4}$ & 4.010 & $2.370 \times 10^{-4}$ & 3.950 \\
\hline 4096 & $3.549 \times 10^{-5}$ & 4.014 & $6.000 \times 10^{-5}$ & 4.000 \\
\hline 3152 & $8.842 \times 10^{-6}$ & 4.046 & $1.500 \times 10^{-5}$ & 3.750 \\
\hline 16384 & $2.185 \times 10^{-6}$ & 4.114 & $4.000 \times 10^{-6}$ & 4.000 \\
\hline 32768 & $5.311 \times 10^{-7}$ & $\mathrm{NA}$ & $1.000 \times 10^{-6}$ & $\mathrm{NA}$ \\
\hline \hline
\end{tabular}

results suggest $\mathcal{O}\left[\delta^{\dagger}\right]$ convergence in that norm. As a reference, 'Table 4.10 compares coarser finite difference-computed solutions with the fine finite difference solution computed on a grid sized $N=32768$. For coarser grid sizes away from $N=32768$ (by three orders of magnitude or more), the trend of $\left\|E^{(N)}\right\|\left\|_{\ell_{1}} /\right\| E^{(i / N)} \|_{\ell_{1}} \approx 4$ and $\left\|E^{(N)}\right\|_{\ell_{\infty}} /\left\|E^{(2 N)}\right\|_{\ell_{\infty}} \approx 4$ is apparent..$^{\circ}$

\footnotetext{
${ }^{8}$ Here, $E^{(N)}$ is the difference between conrser and the finest finite difference computations, where the finest computation is rendered pointwise to the coarser grids.
} 


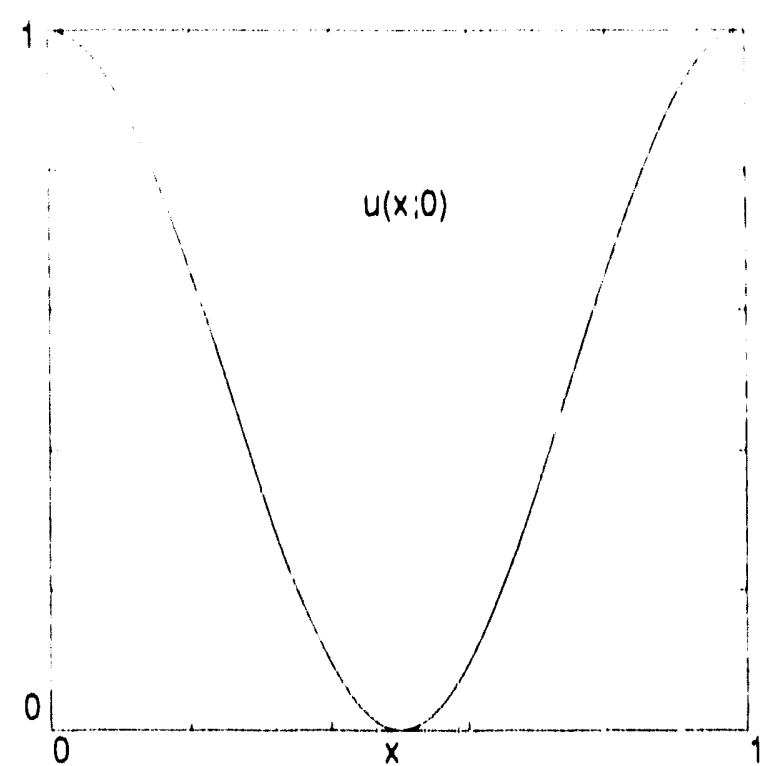

(a)

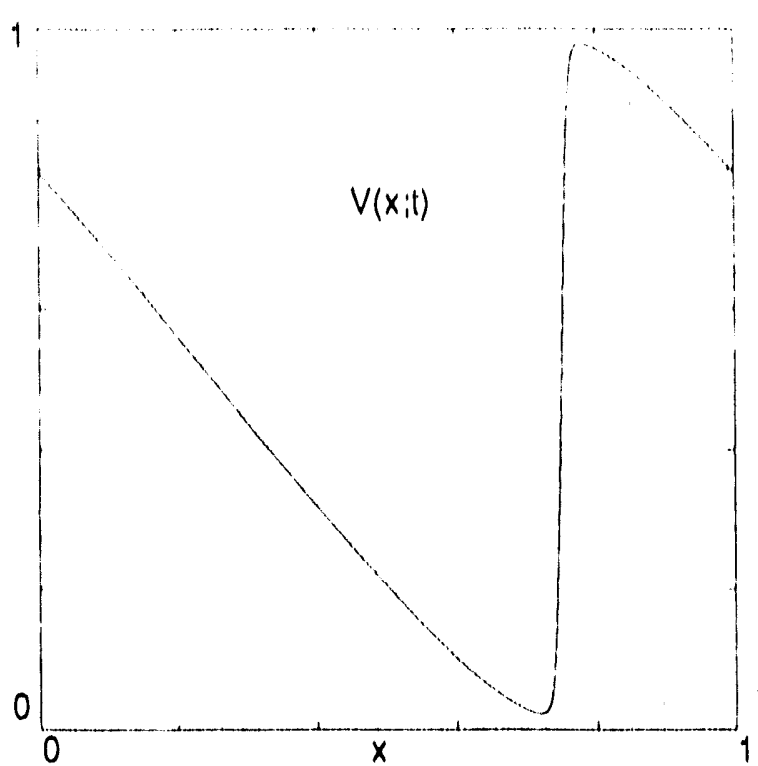

(b)

Figure 4.5: Evolution of $u(x ; t)$ according to Eq. 3.34 with $\nu=2^{-8}$ : (a) Initial condition $u(x ; 0)=u_{I}(x)=(\cos (2 \pi x)+1) / 2 ;(b) u(x ; t)$ at $t=1 / 4$.

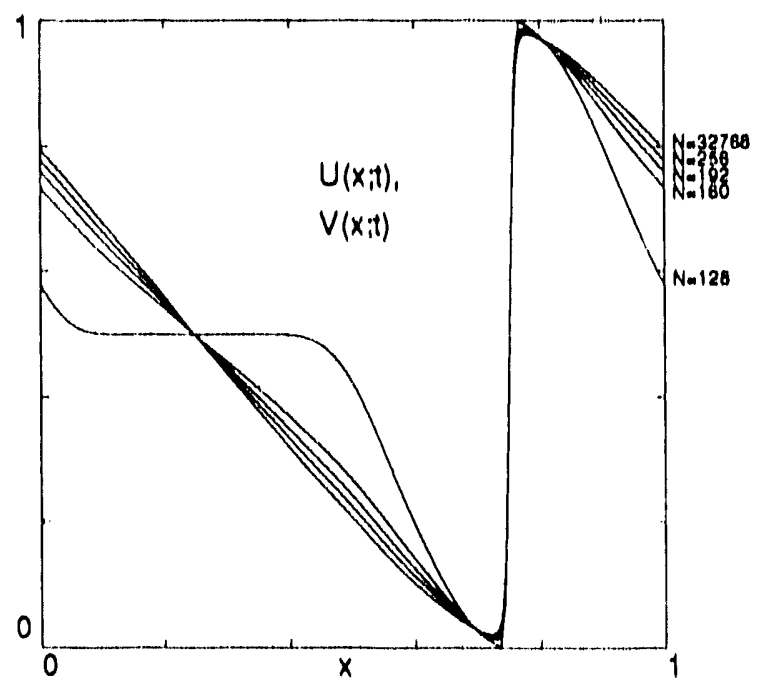

Figure 4.6: Comparison of lattice Boltzmann-computed and finite difference. computed solutions to Eq. 3.34 at $t=1 / 4$ with $\nu=2^{-8}$ and varying grid sizes: The finest grid, i.e, $N=32768$, is the finite difference-computed solution, $V(x, t)$; calculations for grid sizes $N \in\{256,192,160,129\}$ alde the lattice Boltzmann-computed solutions, $U(x, t)$. 
Table 4.10: Comparison of Coarse and Fine Grid Finite Difference-Computed Solutions to Eq. 3.34 at $t=1 / 4$ with $\nu=2^{-8}$ and Initial Condition $u_{I}(x)=$ $(\cos (2 \pi x)+1) / 2$. Fine solution is computed on a 32768 point grid.

\begin{tabular}{|r||c|c||c|c||}
\hline \hline$N$ & $\left\|E^{(N)}\right\|_{\ell_{1}}$ & $\left\|E^{(N)}\right\|_{\ell_{1}} /\left\|E^{(2 N)}\right\|_{\ell_{1}}$ & $\left\|E^{(N)}\right\|_{\ell_{\infty}}$ & $\left\|E^{(N)}\right\|_{\ell_{\infty}}\left\|E^{(2 N)}\right\|_{\ell_{\infty}}$ \\
\hline \hline 256 & $8.990 \times 10^{-4}$ & 4.041 & $6.525 \times 10^{-3}$ & 4.012 \\
\hline 512 & $2.223 \times 10^{-4}$ & 4.009 & $1.626 \times 10^{-3}$ & 4.033 \\
\hline 1024 & $5.551 \times 10^{-5}$ & 4.018 & $4.032 \times 10^{-4}$ & 3.992 \\
\hline 2048 & $1.381 \times 10^{-5}$ & 4.061 & $1.010 \times 10^{-4}$ & 4.040 \\
\hline 4096 & $3.402 \times 10^{-6}$ & 4.207 & $2.500 \times 10^{-5}$ & 4.167 \\
\hline 8192 & $8.087 \times 10^{-7}$ & 4.899 & $6.000 \times 10^{-6}$ & 1.000 \\
\hline 16384 & $1.651 \times 10^{-7}$ & $\infty$ & $6.000 \times 10^{-6}$ & $\infty$ \\
\hline 32768 & $0.000 \times 10^{-0}$ & NA & $0.000 \times 10^{-0}$ & NA \\
\hline \hline
\end{tabular}




\subsection{2-D Advection-Diffusion LB Method}

This section gives some numerical results regarding the order of convergence for lattice Boltzmann method for the two-dimensional advection-diffusion equation, Eq. 3.51. We find that the results suggest $C^{O}\left(\delta^{2}\right)$ convergence for the method in both the $L_{1}$ and $L_{\infty}$-norms for occupations numbers, $n_{k} \in[0,1]$; the theoretical basis for these conclusions remains to be investigated. however. Specifically, we give a finite difference method to generate reference solutions with which to compare the lattice Boltzmann-computed solutions. Then we compare the solutions computed by the two different methods and discuss the results.

\subsubsection{Finite Difference Method}

Consider the conservative finite difference method,

$$
\begin{aligned}
P_{i, j}^{n+1} & =P_{i, j}^{n}-\frac{\Delta t}{4 \Delta x}\left[\left(P_{i+1, j}^{n}\right)^{2}-\left(P_{i-1, j}^{n}\right)^{2}\right] \\
& +\frac{\nu \Delta t}{(\Delta x)^{2}}\left[P_{i+1, j}^{n}-2 P_{i, j}^{n}+P_{i-1, j}^{n}\right]+\frac{\nu \Delta t}{(\Delta y)^{2}}\left[P_{i, j+1}^{n}-2 P_{i, j}^{n}+P_{i, j-1}^{n}\right] .
\end{aligned}
$$

With $\Delta x=\Delta y$, one can shiw that this is an $\mathcal{O}\left[(\Delta x)^{2}\right]+\mathcal{O}[\Delta t]$ monotone finite difference method for solutions $\rho(x, y ; t)$ of Eq. 3.51, which can be transformed to Eq. 3.58 by the linear transformation, $\rho=c(1-2 u)$, with constant $c \geq\|\rho(x, y ; 0)\|_{\ell_{\infty}}$. The stability criterion is that $\Delta t \leq(\Delta x)^{2} /(4 \nu)$. The difference method, Eq. 4.3, can be compared to the lattice Boltzmann method through the transformation,

$$
P_{i, j}^{n}=c\left(1-2 U_{i, j}^{n}\right)
$$




\subsubsection{Numerical Results}

In the absence of a convergence theory for the lattice Boltzmann method, we provide some computational evidence of it being an $\mathcal{O}\left[\delta^{2}\right]$ convergent method for occupations numbers $n_{k} \in[0,1]$ and $\mathcal{O}[\delta]$ advection bias. To this end, we compute solutions to Eq. 3.58 on $\Omega=[0,1] \times[0,1]$ with $c=1$, periodic boundary conditions, and two different initial conditions, $u(x, y ; 0)=u_{I}(x, y) \in[0,1]$, where

$$
\begin{aligned}
& u_{I}(x, y)=(\cos (2 \pi x)+1) / 2 \\
& u_{I}(x, y)=(\sin (2 \pi x) \sin (2 \pi y)+1) / 2 .
\end{aligned}
$$

We compute solutions with initial condition, Eq. 4.5(a), for various diffusion coefficients, $\nu$, while with initial condition, Eq. 4.5(b), for only $\nu=2^{-5}$. Figure 4.7 exhibits the initial conditicn, Eq. 4.5(a), along with with its evolution at time $t=1 / 8$ for $\nu=2^{-5}$. Similarly, Figure 4.8 exhibits the initial condition, Eq. 4.5(b), along with with its evolution at time $t=1 / 8$ for $\nu=2^{-5}$. In both figures the evolution of $U$ is computed on a $512 \times 512$ grid by the finite difference method, Eq. 4.3, through linear transformation, Eq. 4.4 with $c=1$. To avoid conflict with the limit of stability in the finite difference computations, the associated time increment, $\Delta_{F D} t$, is half that limit, i.e., $\Delta_{F D} t=(\Delta x)^{2} /(8 \nu)$.

\section{Quantitative Analysis}

Case $u_{I}(x, y)=(\cos (2 \pi x)+1) / 2:$ Tables $4.11,4.12$, and 4.13 list quantitative results at $t=1 / 8$ for $\nu=2^{-5}: 2^{-4}$, and $2^{-3}$, respectively. In general, for each case the ratio $\left\|E^{(N)}\right\|_{\ell_{1}} /\left\|E^{(2 N)}\right\|_{\ell_{1}}$ is close to four (and approaches four from below for larger $N)$, suggesting an $\mathcal{O}\left[\delta^{2}\right]$ convergence in the $L_{1}$-norm. Although the magnitude of the error in the $L_{\infty}$-norm is greater than in $L_{1}$-norm, the same trend 
holds for the ratio $\left\|E^{(N)}\right\|_{\ell_{\infty}} /\left\|E^{(2 N)}\right\|_{r_{\infty}}$. Hence, $\mathcal{O}\left[\delta^{2}\right]$ convergence in the $L_{\infty}$ norm seems indicated also. Exceptional entries in the tables involving $N=512$ can be explained by noting that the finite difference calculation is $\mathcal{O}\left[(1 / 512)^{2}\right]$ accurate, and therefore can no longer be considered, for comparison purposes, an exact solution. This does not rule out, however, possible superconvergence as the advection bias, $\epsilon$, diminishes to $\epsilon \ll \nu$. (It could also be that some special cancelling of errors occurs for $\nu=2^{-4}$.) While further results would be needed to resolve this matter, it does appear that this lattice Boltzmann method is at least $\mathcal{O}\left[\delta^{2}\right]$ convergent.

Considering the results in the tables as a function of $\nu$, one sees that the magnitude of the error (in both of the norms) decreases for each $N$ as $\nu$ increases. This trend agrees with the predicted theory since the $\mathcal{O}[\delta]$ assumption regarding advection bias in the lattice Boltzmann method is stronger with larger $\nu$.

Case $u_{I}(x, y)=(\sin (2 \pi x) \sin (2 \pi y)+1) / 2$ : Table 4.14 comprises quantitative results for $\nu=2^{-5}$ at $t=1 / 8$. The results show a strong case for $\mathcal{O}\left[\delta^{2}\right]$ convergence in the $L_{1}$-norm, while not as strongly for $\mathcal{O}\left[\delta^{2}\right]$ convergence in the $L_{\infty}$-norm.

In conclusion, based on the quantitative results in Tables 4.11-4.14, it seems likely that at least a theoretical statement regarding $\mathcal{O}\left[\delta^{2}\right]$ convergence in the $L_{1}$-norm for the lattice Boltzmann method for the two-dimensional advection-diffusion equation, Eq. 3.58, with periodic boundary conditions can be obtained; perhaps one in the $L_{\infty}$-norm also. Unfortunately, the results can not offer any prediction of the extent of the domain of monotonicity of the lattice Boltzmann method. 

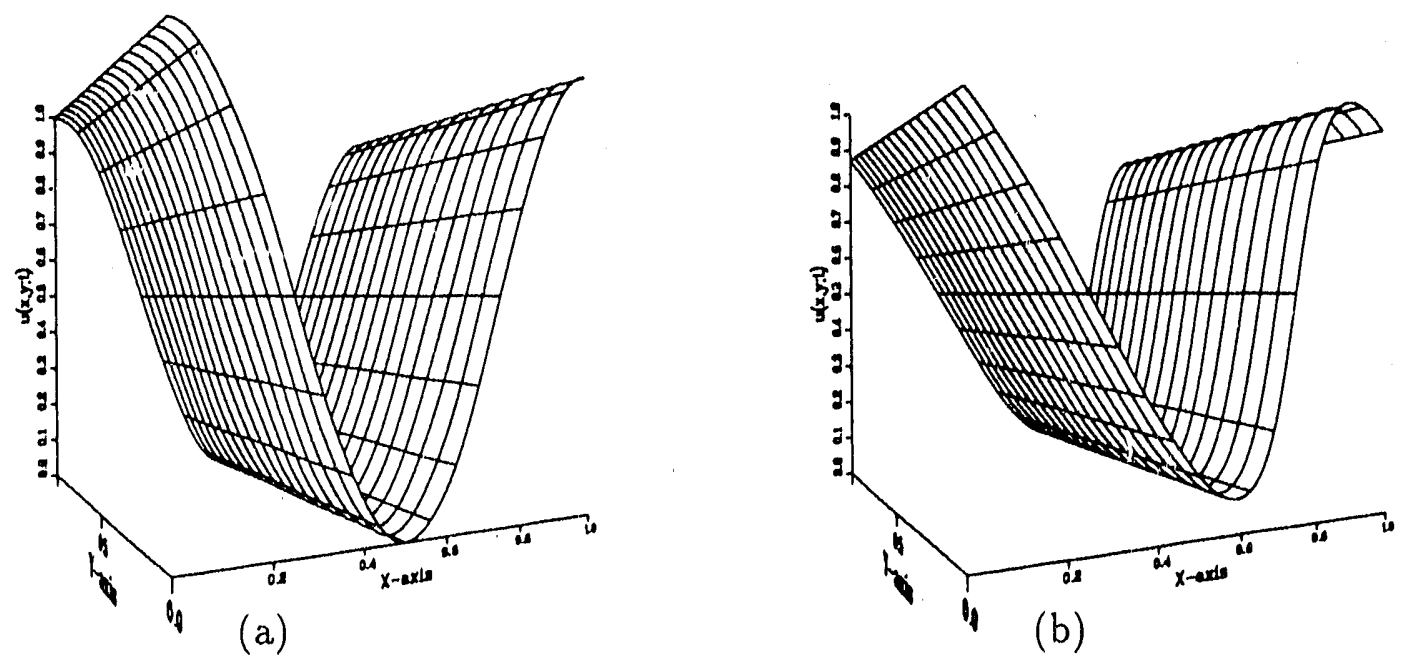

(b)

Figure 4.7: Evolution of $u(x, y ; t)$ according to Eq. 3.51 with $\nu=2^{-5}$ : (a) Initial condition, $u(x, y ; 0)=u_{I}(x, y)=(\cos (2 \pi x)+1) / 2 ;(\mathrm{b}) u(x, y ; t)$ at $t=1 / 8$.

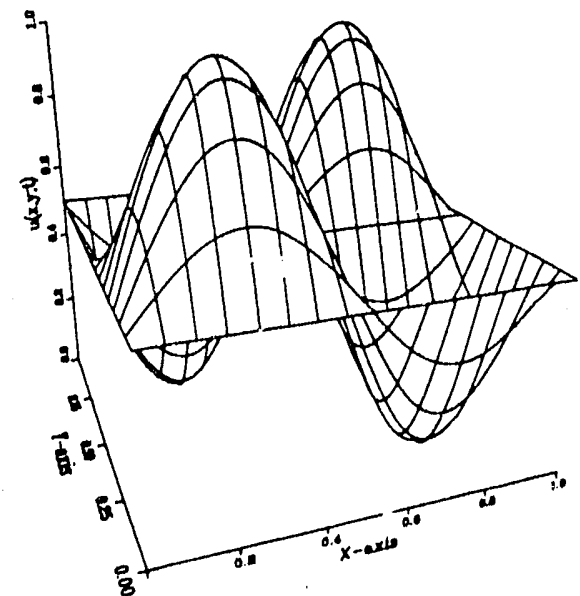

(a)

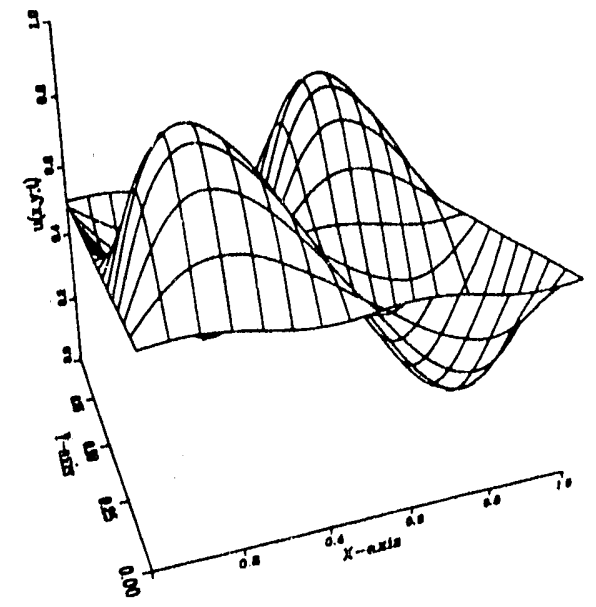

(b)

Figure 4.8: Evolution of $u(x, y ; t)$ according to Eq. 3.51 with $\nu=2^{-5}$ : (a) Initial condition, $u(x, y ; 0)=u_{I}(x, y)=(\sin (2 \pi x) \sin (2 \pi y)+1) / 2$; (b) $u(x, y ; t)$ at $t=1 / 8$. 
Table 4.11: Comparison Lattice Boltzmann- and Finite Difference-Computed Solutions to Eq. 3.58 at $t=1 / 8$ for $\nu=1 / 32$ and Initial Condition $(\cos (2 \pi x)+1) / 2$.

\begin{tabular}{|c|c|c|c|c|c|}
\hline$N^{\prime}$ & $1 / N^{2}$ & $\left\|E^{(N)}\right\|_{\ell_{1}}$ & $\left\|E^{(N)}\right\|_{\ell_{1}} /\left\|E^{\left(2 N^{\prime}\right)}\right\|_{\ell_{1}}$ & $\left\|E^{\left(N^{\prime}\right)}\right\|_{\ell_{\infty}}$ & $\left\|E^{(N)}\right\|_{\ell_{\infty}} /\left\|E^{(2 N)}\right\|_{\ell}$ \\
\hline 32 & $1 / 1024$ & 0.00986 & 3.446 & 0.0327 & 3.287 \\
\hline 64 & $1 / 4096$ & 0.00286 & 3.698 & 0.00996 & 3.563 \\
\hline 128 & $1 / 16384$ & 0.000774 & 3.836 & 0.00280 & 3.813 \\
\hline 256 & $1 / 65536$ & 0.000202 & 3.973 & 0.000733 & 3.899 \\
\hline 512 & $1 / 262144$ & 0.0000508 & $\mathrm{NA}$ & 0.000188 & NA \\
\hline
\end{tabular}

\subsection{Conclusions}

We have presented computational results substantiating the theoretical results in the $L_{1}$-norm that $\mathrm{LB}_{1}, \mathrm{LB}_{2}$, and the lattice method for the one-dimensional Burgers' equation are $\mathcal{O}\left[\delta^{2}\right]$ convergent monotone finite difference methods in the respective domains of convergence. Additional computational results support the conjecture that the lattice method for the two-dimensional advection-diffusion equation, Eq. 3.51, is $\mathcal{O}\left[\delta^{2}\right]$ convergent in the $L_{1}$-norm. 
Table 4.12: Comparison of Lattice Boltzmann- and Finite Difference-Computed Solutions to Eq. 3.58 at $t=1 / 8$ for $\nu=1 / 16$ and Initial Condition $(\cos (2 \pi x)+1) / 2$.

\begin{tabular}{||cr||c|c||c|c||}
\hline \hline$N$ & $1 / N^{2}$ & $\left\|E^{(N)}\right\|_{\ell_{1}}$ & $\left\|E^{(N)}\right\|_{\ell_{1}}\left\|E^{(2 N)}\right\|_{\ell_{1}}$ & $\left\|E^{(N)}\right\|_{\ell_{\infty}}$ & $\left\|E^{(N)}\right\|_{\ell_{\infty}} /\left\|E^{(2 N)}\right\|_{\ell_{\infty}}$ \\
\hline \hline 32 & $1 / 1024$ & $3.85 \times 10^{-3}$ & 4.014 & $9.88 \times 10^{-3}$ & 3.896 \\
\hline 64 & $1 / 4096$ & $9.58 \times 10^{-4}$ & 3.967 & $2.54 \times 10^{-3}$ & 4.043 \\
\hline 128 & $1 / 16384$ & $2.42 \times 10^{-4}$ & 4.000 & $6.27 \times 10^{-4}$ & 4.071 \\
\hline 256 & $1 / 65536$ & $6.04 \times 10^{-5}$ & 9.155 & $1.54 \times 10^{-4}$ & 10.27 \\
\hline 512 & $1 / 262144$ & $6.60 \times 10^{-6}$ & $\mathrm{NA}$ & $1.50 \times 10^{-5}$ & $\mathrm{NA}$ \\
\hline \hline
\end{tabular}

Table 4.13: Comparison of Lattice Boltzmann- and Finite Difference-Computed Solutions to Eq. 3.58 at $t=1 / 8$ for $\nu=1 / 8$ and Initial Condition $(\cos (2 \pi x)+1) / 2$.

\begin{tabular}{||cr||c|c||c|c||}
\hline \hline$N$ & $1 / N^{2}$ & $\left\|E^{(N)}\right\|_{\ell_{1}}$ & $\left\|E^{(N)}\right\|_{\ell_{1}} /\left\|E^{(2 N)}\right\|_{\ell_{1}}$ & $\left\|E^{(N)}\right\|_{\ell_{\infty}}$ & $\left\|E^{(N)}\right\|_{\ell_{\infty}} /\left\|E^{(2 N)}\right\|_{\ell_{\infty}}$ \\
\hline \hline 32 & $1 / 1024$ & $9.50 \times 10^{-4}$ & 3.963 & $1.77 \times 10^{-3}$ & 3.582 \\
\hline 64 & $1 / 4096$ & $2.40 \times 10^{-4}$ & 3.789 & $4.93 \times 10^{-4}$ & 3.652 \\
\hline 128 & $1 / 16384$ & $6.32 \times 10^{-5}$ & 3.883 & $1.35 \times 10^{-4}$ & 3.857 \\
\hline 256 & $1 / 65536$ & $1.63 \times 10^{-5}$ & 4.163 & $3.50 \times 10^{-5}$ & 3.889 \\
\hline 512 & $1 / 262144$ & $3.91 \times 10^{-6}$ & $\mathrm{NA}$ & $9.00 \times 10^{-4}$ & $\mathrm{NA}$ \\
\hline \hline
\end{tabular}


Table 4.14: Comparison of Lattice Boltzmann- and Finite Difference-Computed Solutions to Eq. 3.58 at $t=1 / 8$ for $\nu=1 / 32$ and Initial Condition $(\sin (2 \pi x) \sin (2 \pi y)+$ $1) / 2$.

\begin{tabular}{||cr||c|c||c|c||}
\hline \hline$N$ & $1 / N^{2}$ & $\left\|E^{(N)}\right\|_{\| \ell_{1}}$ & $\left\|E^{(N)}\right\|_{\ell_{1}} /\left\|E^{(2 N)}\right\|_{\ell_{1}}$ & $\left\|E^{(N)}\right\|_{\ell_{\infty}}$ & $\left\|E^{(N)}\right\|_{\ell_{0}} /\left\|E^{(2 N)}\right\|_{\ell_{\infty}}$ \\
\hline \hline 32 & $1 / 1024$ & $6.68 \times 10^{-3}$ & 4.181 & $2.38 \times 10^{-2}$ & 3.861 \\
\hline 64 & $1 / 4096$ & $1.60 \times 10^{-3}$ & 4.034 & $6.17 \times 10^{-3}$ & 3.900 \\
\hline 128 & $1 / 16384$ & $3.96 \times 10^{-4}$ & 4.011 & $1.58 \times 10^{-4}$ & 3.923 \\
\hline 256 & $1 / 65536$ & $9.87 \times 10^{-5}$ & 4.009 & $4.03 \times 10^{-4}$ & 3.838 \\
\hline 512 & $1 / 262144$ & $2.46 \times 10^{-5}$ & $\mathrm{NA}$ & $1.05 \times 10^{-4}$ & $\mathrm{NA}$ \\
\hline \hline
\end{tabular}




\section{Chapter 5}

\section{A Domain Decomposition for Lattice Methods}

One difficulty with lattice gas and lattice Boltzmann methods (and often with explicit methods) is that refining the grid for a problern's domain can exorbitantly increase the amount of computation. The stability criterion often appears as a restriction

on the time step, $\Delta t=\mathcal{O}\left[\Delta x^{2}\right] ;$ a twofold increase in the number of grid points corresponds to a fourfold increase in the number of time steps. Were one to refine the grid of such a method, the amount of computation would, depending on the stability criterion and the grid refinement, dramatically increase.

However, often a more accurate computational solution is not required everywhere in the problem domain. It would be desirable to be able to concentrate computational resources in regions of interest, e.g., near boundary layers and shocks. There are techniques available for finite difference methods for accomplishing such redistribution of resources. One technique is domain decomposition, where the problem domain is subdivided into subregions according to some interest metric. Artificial boundary conditions are imposed along the boundaries of the subdomains, which may overlap. And for time-dependent problems these boundaries, as well as the number of subdomains, may change. Ideally, a numerical time differencing scheme is used to advance 
the solution over the entire domain. Then a measurement of the computed soiution is taken to determine subregions of activity: The solution is then recomputed on these subregions using a different, grid and possibly a different time differencing scheme. Initial and boundary conditions on the subdomains are obtained by interpolating the data from the computed solution on the global domain $[60,61,68]$.

For implicit finite difference schemes, a computed solution may involve iterating computations over the subdomains until some convergence criterion is satisfied, e.g., the Schwartz Alternating Method [67]. A typical finite difference scheme does not in general involve iteration. Subregion solutions are simply computed in the subregions with the boundaries determined from neighboring subregions. A reasonable domain decomposition strategy is the following:

1. Advance coarse time step $\Delta_{c} t$ on the coarse lattice.

2. Decompose problem dornain into subregions.

3. Interpolate coarse boundaries to obtain boundary conditions for refined regions.

4. Advance refined subregions a number of time steps to correspond with one coarse time step.

5. Update coarse lattice.

The purpose of this chapter is to investigate the possibility and plausibility of sub-structuring schemes (domain decomposition) for lattice gas methods. We provide a proof of concept of using sub-structuring techniques to better utilize consputational resources in these methods. We forego attempting to generate subregions from computed solutions and assume a static domain decomposition. Further, we 
do not update the coarse lattice solution based upon the fine lattice solution. Sub. structuring is shown to have some viability with this simplification for the lattice gas method discussed in Section 3.2. It indicates the plausibility of using some form of sub-structuring in lattice gas methods.

Section 5.1 proposes a sub-structuring method for lattice gas method of Section 3.2. Section 5.2 provides supporting computational evidence. Finally, general conclusions are drawn in Section 5.3.

\subsection{A Sub-Structuring Method for a Lattice Gas Method for the 1-D Viscous Burgers Equa- tion}

Consider the lattice gas method discussed in Section 3.2 for the one-dimensional viscous Burgers equation. Ideally in a sub-structuring method for this lattice gas method, the mean occupation numbers would be computed on a coarse lattice and then a first order measurement of a gradient depending on them would be taken to determine active subregions. Initial and boundary conditions on these subregions would be obtained by interpolating the computed mean occupation numbers on the coarse lattice. The mean occupation numbers would then be recomputed on these subregions using a finer lattice. And the process would repeat at time $\hat{\imath}+\Delta_{c} t$. (Quantities related to the differing lattices are subscripted or superscripted with a "c" for coarse lattice and " $f$ " for fine lattice.)

Desiring a proof of concept for using domain decomposition in lattice gas methods methods, we consider a problem with a stationary shock so that a static domain 
decomposition may be used. In a typical finite difference calculation (without domain (lecomposition), computing solutions with a higher resolution requires a finer mesh. The question to be answered by our study is whether it seems reasonable to expect that a domain decomposition strategy is viable (at all) in a lattice gas setting.

Consider a coarse periodic lattice $\mathfrak{L}_{c}$ with lattice spacing $\Delta_{c} x$ on a domain $\Omega=$ $[0,1]$. Then $\Delta_{c} t=\left(\Delta_{c} x\right)^{2} /(2 \nu)$. The advection bias is given by $\epsilon_{c}=\Delta_{c} x /(2 \nu)$ (assuming $c=1 \mathrm{in} \mathrm{Eq} .3 .35)$. Suppose refinement is desired on $\Omega_{f}=\left[B_{L}, B_{R}\right]$ for $B_{L}<B_{R}$. Let us associate a refined lattice $\mathfrak{L}_{f}$ on $\Omega_{f}$ with lattice spacing $\Delta_{f} x$ such that $\Delta_{f} x=\Delta_{c} x / 2$. Then $\Delta_{f} t=\Delta_{c} t / 4$ and $\epsilon_{f}=2 \epsilon_{c}$. Note that $\epsilon_{f}=\mathcal{O}\left[\Delta_{c} x\right]$ still. We have two sets of occupation numbers.

The coarse lattice maintains $n_{k}^{(c)}(x ; t)$ for $k \in\{0,1\}$, where $x \in[0,1]$ is an integral multiple of $\Delta_{c} x$ and $t$ is an integral multiple of $\Delta_{c} t$. The fine lattice maintains $n_{k}^{(f)}(x ; t)$ for $k \in\{0,1\}$, where $x \in\left[B_{L}, B_{R}\right]$ is an integral multiple of $\Delta_{f} x$ and $t$ is an integral multiple of $\Delta_{f} t$. In our approach, the fine solution depends on the coarse lattice for its boundaries; the coarse solution operates independently. Initially, $n_{k}^{(c)}$ and $n_{k}^{(f)}$ is determined from the initial condition of the problem. Then $n_{k}^{(c)}$ at future time steps is determined by the lattice gas method with $\epsilon=\epsilon_{c}$. To determine $n_{k}^{(f)}\left(B_{L / R} ; m_{f} \Delta_{f} t\right)$, we advance $n_{k}^{(c)}$ one $\Delta_{c} t$ time step, from $m_{c} \Delta_{c} t$ to $\left(m_{c}+\right.$ 1) $\Delta_{c} t$. Then we interpolate the ensemble averaged values of $n_{k}^{(c)}\left(B_{L / R} ; m_{c} \Delta_{c} t\right)$ and $n_{k}^{(c)}\left(B_{L / R} ;\left(m_{c}+1\right) \Delta_{c} t\right)$ to obtain Dirichlet boundary conditions for $n_{k}^{(f)}\left(B_{L / R} ; m_{c} \Delta_{c} t+\right.$ $\left.j \Delta_{f} t\right)$, where $j \in\{0,1,2,3,4\}$. Then $n_{k}^{(f)}(x ; t)$ for $x \in\left(B_{L}, B_{R}\right)$ and $t=m_{c} \Delta_{c} t+j \Delta_{f} t$ for $j \in\{1,2,3,4\}$ is determined by operating the lattice gas method with $\epsilon=\epsilon_{f}$. The process repeats. Note that this algorithm operates on an ensernble of lattice gas instances so that the interpolated boundaries are more accurately represented. The 
results of the computation are (1) a conrse solution, which is identical to the result without the decomposition, and $(2)$ a fine solution on $\Omega_{f}$.

\subsection{Computational Evidence}

We apply the lattice gas method of Section 3.2 to compute approximate solutions to Eq. 3.29 with periodic boundary conditions and initial condition

$$
\rho_{I}(x)=\rho(x ; 0)= \begin{cases}-\frac{4}{5}+\frac{1}{5}, & 0 \leq x<3 / 4 \\ -\frac{4}{5}+1, & 3 / 4 \leq x \leq 1\end{cases}
$$

where $0 \leq x \leq 1$ and

$$
\nu=2^{-\theta}
$$

Then the initial condition for the lattice gas method is given by

$$
n_{k}\left(x_{i} ; 0\right)= \begin{cases}1, & \text { with probability } \rho_{I}\left(x_{i}\right) / 2 \\ 0, & \text { with probability } 1-\rho_{I}\left(x_{i}\right) / 2\end{cases}
$$

where $x_{i}=i \Delta_{c} x \in[0,1]$ or $x_{i}=i \Delta_{f} x \in\left[B_{L}, B_{R}\right]$, depending on whether the coarse or fine lattices are being initialized.

Let $n_{k_{1}(j)}(x ; t) \in \mathcal{B}$ denote lattice gas instance $j$. Then the ensemble averages in our computation are

$$
N_{k}(x ; t)=\left\langle n_{k,(j)}(x ; t)\right\rangle=\frac{1}{P} \sum_{j=1}^{P} n_{k,(j)}(x ; t),
$$

where $P=2048$ is the number of lattice gas instances in the ensemble. (The $(x ; t)$ are computed only at the discrete spatial and temporal locations.) Let

$$
\mathcal{N}(x ; t)=N_{0}(x ; t)+N_{1}(x ; t)
$$


Our results also include interplay with some spalial aleraging. Let

$$
\hat{\mathcal{N}}(x ; t)=\frac{1}{M} \sum_{n=0}^{M-1} N(x+\mu \Delta x ; t) .
$$

Then our npproximation to $p$ is

$$
\hat{\rho}=\hat{N}(x ; t) / 2-1
$$

Regarding the figures, the horizontal axis is the spatial dimension a $\in(0,1)$; the vertical axis is $\hat{\rho} \in(-1,+1)$. Figure 5.1 depicts the initial condition. The remaining figures (Figures $5.2-5.5$ ) exhibit a conservative finite difference calculation ${ }^{1}$ superimposed on coarse and fine solutions at $t=0.125$. The figures vary with the level of spatial averaging. In each case, the fine solution more accurately capturing the steep gradient near $x=3 / 4$. And as one expects, increased spatial averaging decreases higher frequencies in the noise while also smoaring the steep gradient near $x=3 / 4$. The steep gradient is better resolved on the fine lattice over the region $\Omega_{f}$ without having to compute a fine solution everywhere on $\Omega$.

\subsection{Conclusions}

Using ideas developed in [60] for solving Burgers' equation by domain decomposition, a domain decomposition method was developed for a lattice gas method for the onedimensional viscous Burgers equation. The computational evidence supports that sub-structuring or decomposing lattice gas methods is a viable means for achieving greater accuracy in specific regions without having to pay for an everywhere reduced performance.

\footnotetext{
'Appendix (' describes the method used.
} 


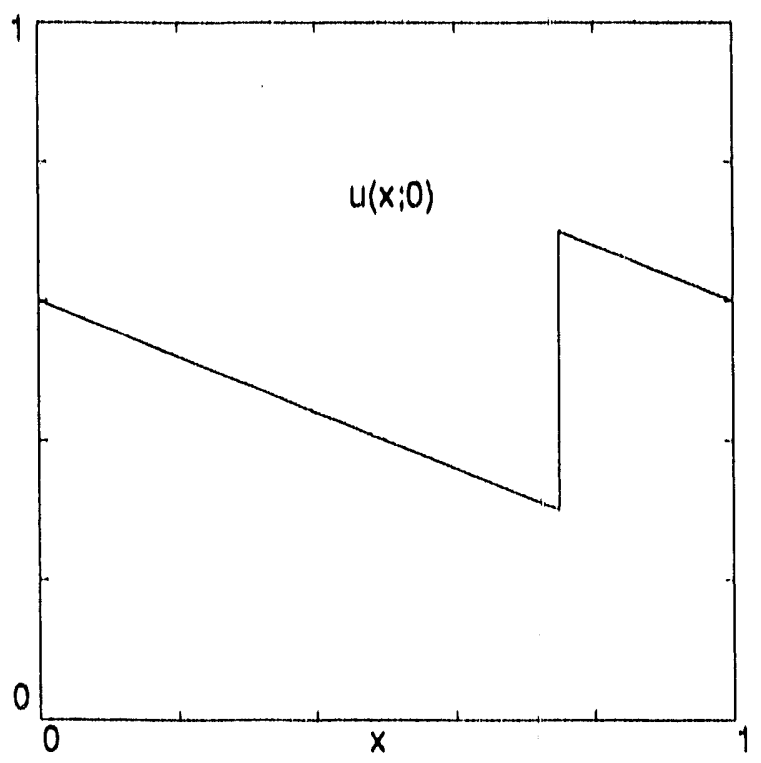

Figure 5.1: Initial conditioı.

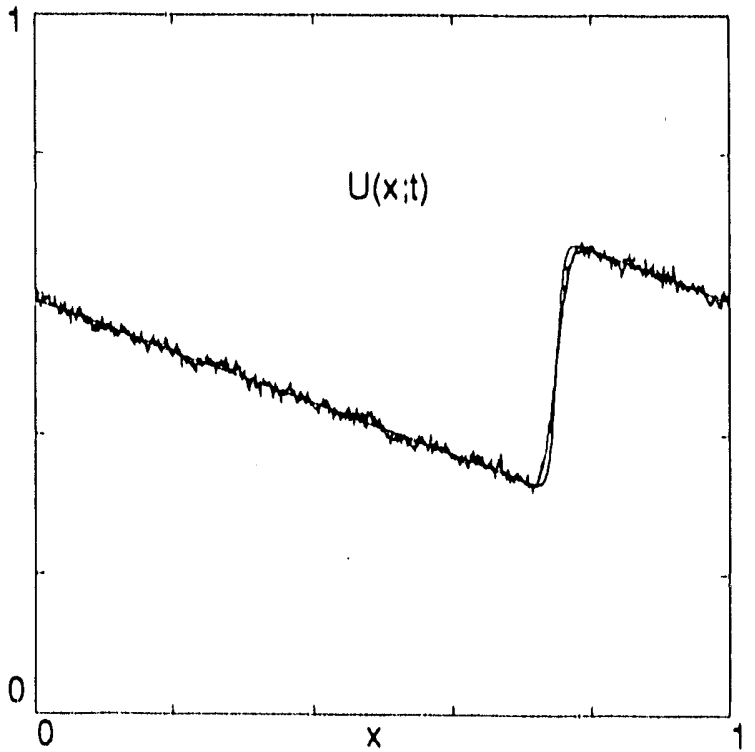

(a)

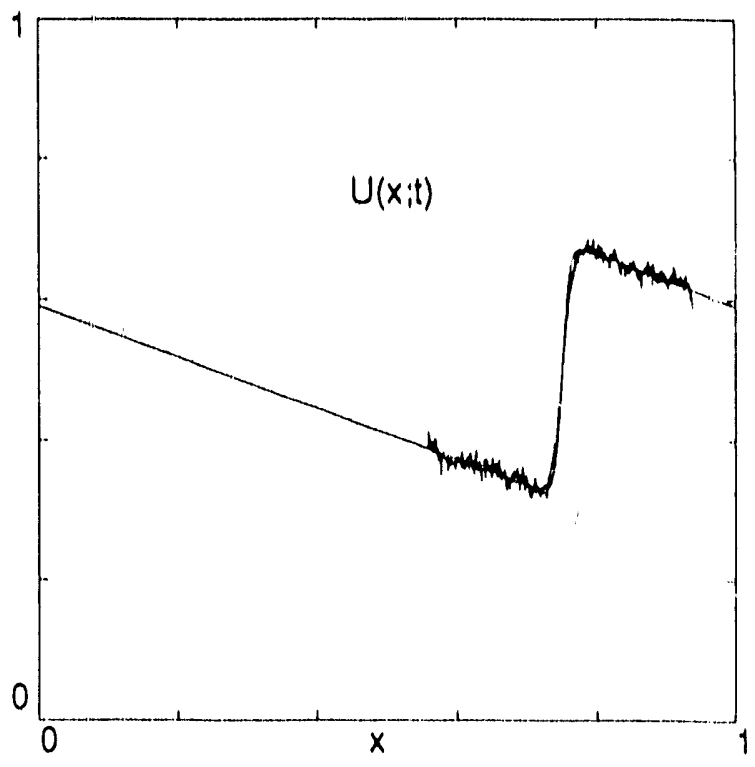

(b)

Figure 5.2: (a) Coarse lattice and (b) fine lattice solutions; $t=0.125 ; M=1$. 


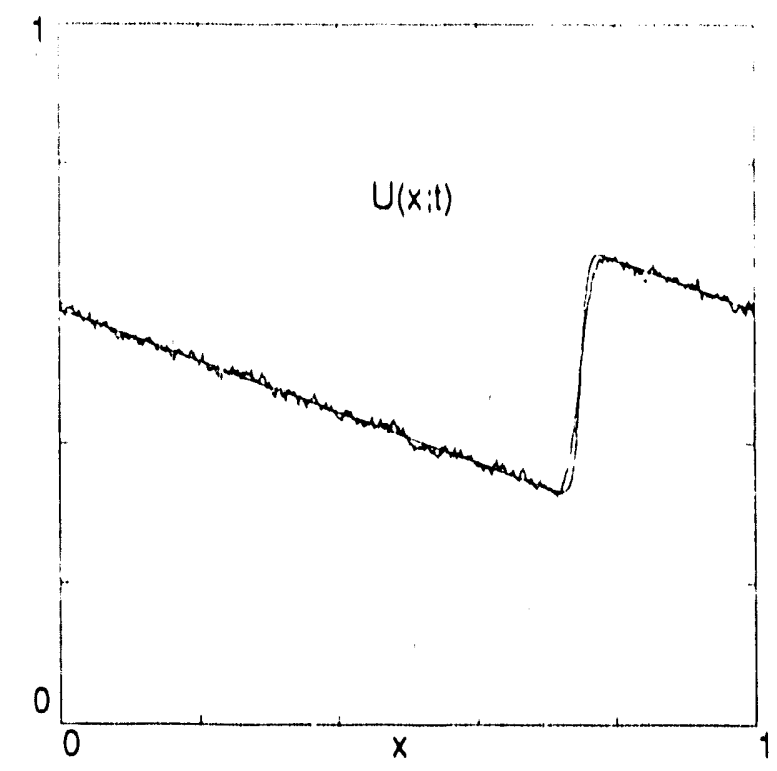

(a)

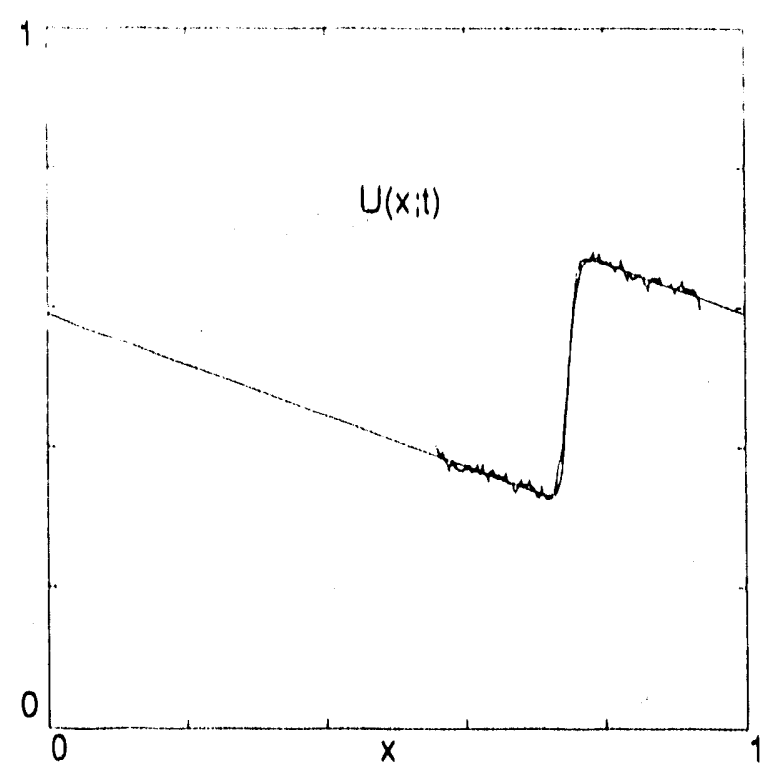

(b)

Figure 5.3: (a) Coarse lattice and (b) fine lattice solutions; $t=0.125 ; M=2$.

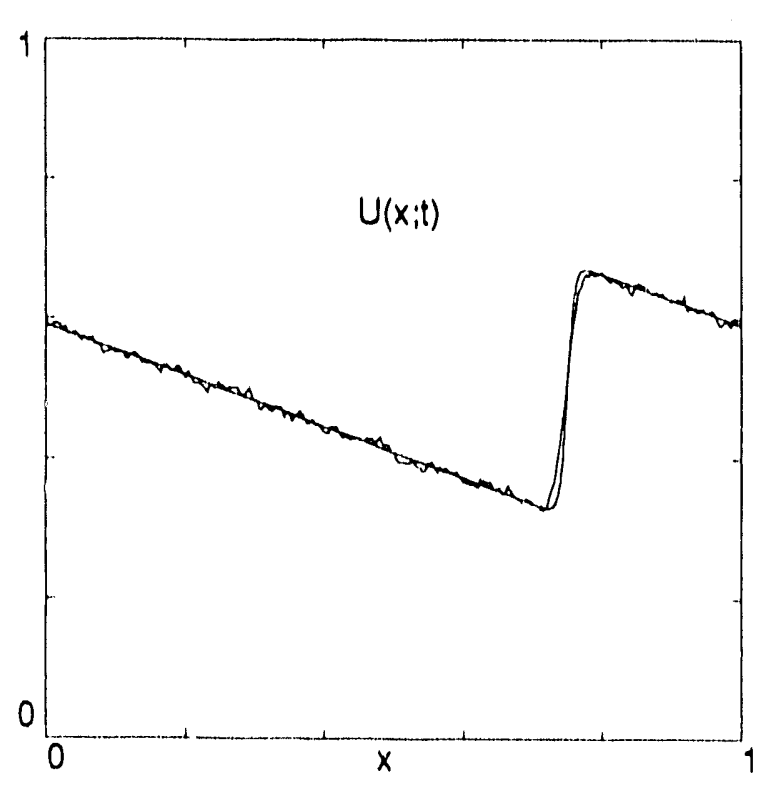

(a)

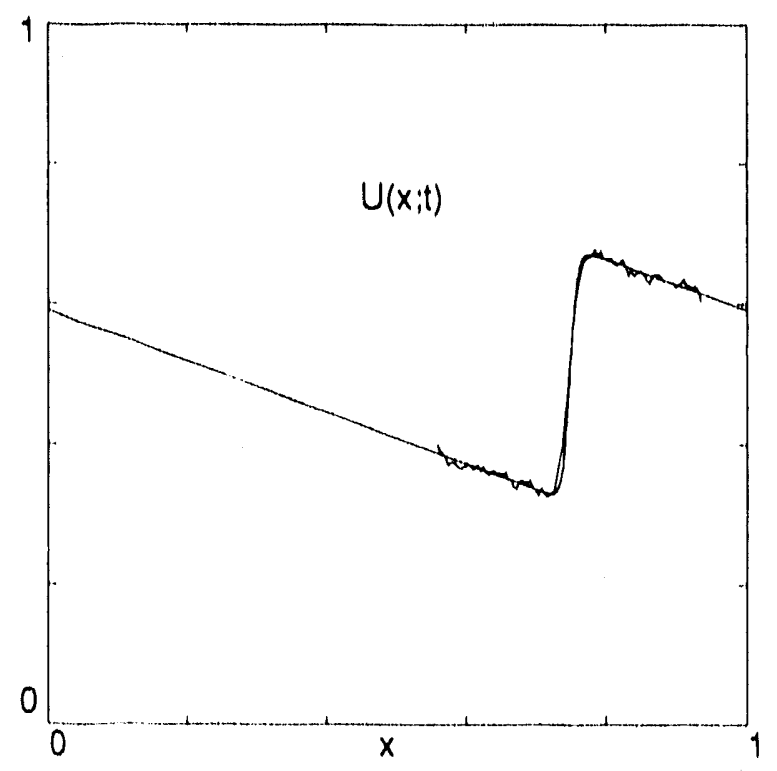

(b)

Figure 5.4: (a) Coarse latice and (b) fine lattice solutions; $t=0.125 ; M=4$. 


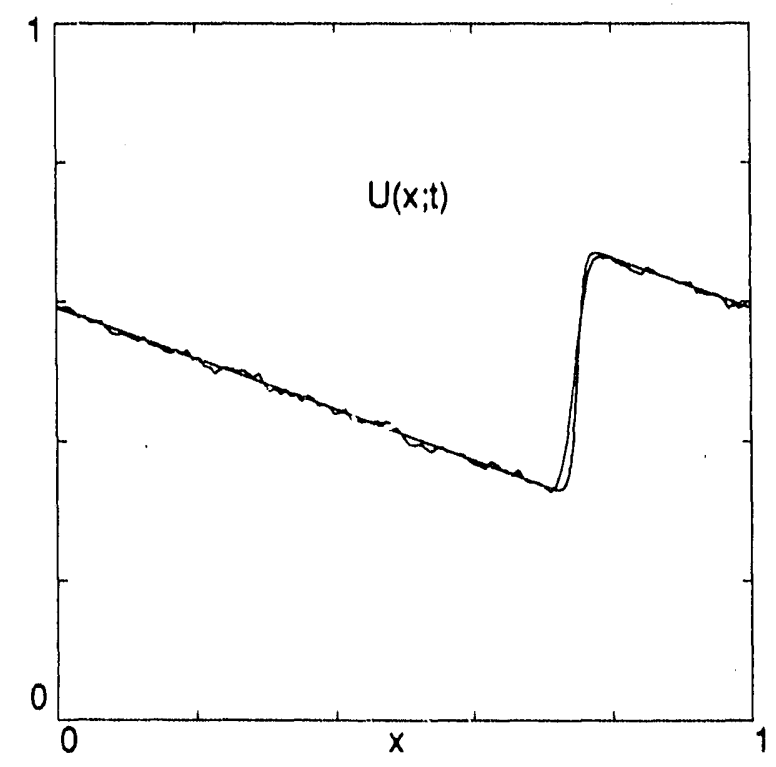

(a)

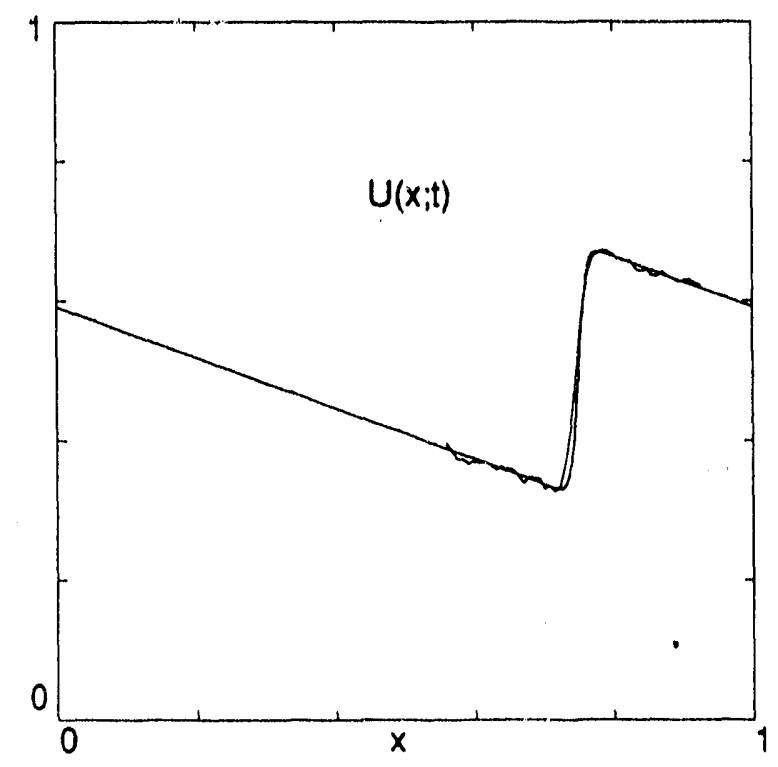

(b)

Figure 5.5: (a) Coarse lattice and (b) fine lattice solutions; $t=0.125 ; M=8$. 


\section{Chapter 6 Conclusions}

This dissertation provides a theoretical basis for studying the numerical properties regarding consistency, stability, and convergence of lattice Boltzmann methods and the Lattice Boltzmann Approximation to lattice gas methods for computationally solving some partial differential equations. The lattice methods studied are shown to be second-order explicit, conservative, monotone finite difference methods. Convergence proofs were provided for lattice methods for two nonlinear two-dimensional diffusion models of the form,

$$
u_{t}=\nu \nabla \cdot(D(u) \nabla u)
$$

in which $D(u)$ is a nonlinear diffusion coefficient determined by the discrete ChapmanEnskog expansion, and one nonlinear one-dimensional advection-diffusion model (the one-dimensional viscous Burgers equation),

$$
\rho_{t}+\rho \rho_{x}=\nu \rho_{x x}
$$

The details of the convergence proofs revealed possible restrictions on the occupation numbers for convergence of a lattice method. Computational evidence that compares lattice Boltzmann- and finite difference-computed solutions substantiated the results. 
This could have significant repercussions for lattice methods in general. The computations, completed on Alliant $1: \mathrm{X} / \mathrm{S}, \mathrm{CRAY}-2, \mathrm{CRAY}-\mathrm{X} / \mathrm{MP}$, and Connection Machine CM-2 computers, included a variety of initial conditions. While convergence was not proven for a lattice method for a nonlinear two-dimensional advection-diffusion equation,

$$
\rho_{t}+\rho \rho_{x}=\nu\left(\rho_{x x}+\rho_{y y}\right)
$$

computational evidence suggests second-order convergence.

A domain decomposition strategy for lattice gas methods was introduced. The strategy uses a combination of coarse and fine lattices to resolve regions of interest, e.g., near steep gradients, with fewer computational resources than a case in which only a fine lattice is used. Toward developing a proof of concept, the ideas were applied to the lattice gas method for the one-dimensional viscous Burgers equation. For simplification the problem involved a stationary steep gradient. Computational results verified that a finer resolution near a steep gradient can be obtained with the combined coarse and fine lattices than with only the coarse lattice. The results indicate that the strategy shows some promise and merits further investigation. Although the strategy was originally developed for lattice gas methods, it can apply to lattice Boltzmann methods.

Generally, it is expected that the numerical theory will extend to other lattice methods, e.g., the FHP [38] and FCHC [37] type models for the two- and threedimensional Navier-Stokes equations, respectively. The extension would also include lattice Boltzmann methods lacking the Fermi Exclusion Principle. Convergence of these and other lattice methods remains to be investigated in an effort to build the numerical understanding of lattice methods and to bring them into a better position 
for competition with other algorithms. 


\section{Appendix A}

\section{$\mathrm{LB}_{1}$ Analysis Details}

This appendix lists some of the details regarding the discrete Chapman-Enskog analysis of the lattice Boltzmann method, $\mathrm{LB}_{1}$. The listing is order by order as in Section 2.3.5. We denote zero order parameter $\sigma^{(0)}=u$, and also use

$$
\begin{aligned}
D(u) & =-\frac{1}{\lambda}-\frac{1}{2}=\frac{1}{4 u(1-u)}-\frac{1}{2}, \\
D^{\prime}(u) & =\frac{4(2 u-1)}{\lambda^{2}},
\end{aligned}
$$

in which $\lambda=-4 u(1-u)$. Also, regarding notation, at $\mathcal{O}\left[\delta^{4}\right]$, we do use some of the $c_{k}^{(j)}$ and $\tilde{c}_{k}^{(j)}$ coefficients as defined in Section 2.3.5; otherwise, the expressions would be undescriptively long.

$\mathcal{O}[\delta]:$

$\left[\left(\vec{e}_{k} \cdot \nabla\right) n_{k}^{(0)}\right]=\mathrm{q}_{1} u_{x}+\mathrm{q}_{2} u_{y}$.

$\mathcal{O}\left[\delta^{2}\right]:$

$$
\begin{aligned}
& {\left[\left(\vec{e}_{k} \cdot \nabla\right)^{2} n_{k}^{(0)}\right]=\mathrm{q}_{0} \frac{1}{2}\left(u_{x x}+u_{y y}\right)+\mathrm{q}_{3} \frac{1}{2}\left(u_{x x}-u_{y y}\right),} \\
& {\left[\frac{\partial}{\partial t} n_{k}^{(0)}\right]=\mathrm{q}_{0} u_{t},}
\end{aligned}
$$




$$
\begin{aligned}
& {\left[\left(\vec{e}_{k} \cdot \nabla\right) n_{k}^{(1)}\right]} \\
& =\mathbf{q}_{0} \frac{L}{2}\left(\frac{\partial}{\partial x}\left(\frac{1}{\lambda} u_{x}\right)+\frac{\partial}{\partial y}\left(\frac{1}{\lambda} u_{y}\right)\right)+\mathbf{q}_{1} \sigma_{x}^{(1)}+\mathbf{q}_{2} \sigma_{y}^{(1)} \\
& +\mathbf{q}_{3} \frac{L}{2}\left(\frac{\partial}{\partial x}\left(\frac{1}{\lambda} u_{x}\right)-\frac{\partial}{\partial y}\left(\frac{1}{\lambda} u_{y}\right)\right) \\
& \mathcal{D}^{2} \mathcal{C}\left(\mathbf{n}^{(0)}\right) \cdot \mathbf{n}^{(1)} \mathbf{n}^{(1)} \\
& =\mathbf{q}_{1} 2 L \lambda \sigma^{(1)} D^{\prime}(u) u_{x}+\mathbf{q}_{2} 2 L \lambda \sigma^{(1)} D^{\prime}(u) u_{y}-\mathbf{q}_{3} \frac{L^{2}}{2} D^{\prime}(u)\left(\left(u_{x}\right)^{2}-\left(u_{y}\right)^{2}\right)
\end{aligned}
$$

$\mathcal{O}\left[\delta^{3}\right]:$

$$
\begin{aligned}
& {\left[\left(\vec{e}_{k} \cdot \nabla\right)^{3} n_{k}^{(0)}\right]=\mathrm{q}_{1} u_{x x x}+\mathrm{q}_{2} u_{y y y},} \\
& {\left[\left(\vec{e}_{k} \cdot \nabla\right) \frac{\partial}{\partial t} n_{k}^{(0)}\right]=\mathrm{q}_{1} u_{x t}+\mathrm{q}_{2} u_{y t}} \\
& {\left[\left(\vec{e}_{k} \cdot \nabla\right)^{2} n_{k}^{(1)}\right]} \\
& =\mathrm{q}_{0} \frac{1}{2}\left(\sigma_{x x}^{(1)}+\sigma_{y y}^{(1)}\right)+\mathrm{q}_{1} L \frac{\partial^{2}}{\partial x^{2}}\left(\frac{1}{\lambda} u_{x}\right)+\mathrm{q}_{2} L \frac{\partial^{2}}{\partial y^{2}}\left(\frac{1}{\lambda} u_{y}\right)+\mathrm{q}_{3} \frac{1}{2}\left(\sigma_{x x}^{(1)}-\sigma_{y y}^{(1)}\right), \\
& \frac{\partial}{\partial t} \mathbf{n}^{(1)}=\mathbf{q}_{0} \sigma_{t}^{(1)}+\mathbf{q}_{1} L \frac{\partial}{\partial t}\left(\frac{1}{\lambda} u_{x}\right)+\mathbf{q}_{2} L \frac{\partial}{\partial t}\left(\frac{1}{\lambda} u_{y}\right) \\
& {\left[\left(\vec{e}_{k} \cdot \nabla\right) n_{k}^{(2)}\right]} \\
& =\mathrm{q}_{0} \frac{L}{2} \nabla \cdot\left[\frac{1}{\lambda} \nabla \sigma^{(1)}-D^{\prime}(u) \sigma^{(1)} \nabla u\right] \\
& +\mathrm{q}_{1}\left[\sigma_{x}^{(2)}+\frac{L^{2}}{2} \frac{\partial}{\partial x}\left(\frac{1}{2 \lambda} D^{\prime}(u)\left(\left(u_{x}\right)^{2}-\left(u_{y}\right)^{2}\right)-\frac{1}{\lambda}\left(\frac{\partial}{\partial x}\left(D(u) u_{x}\right)-\frac{\partial}{\partial y}\left(D(u) u_{y}\right)\right)\right)\right] \\
& +\mathbf{q}_{2}\left[\sigma_{y}^{(2)}-\frac{L^{2}}{2} \frac{\partial}{\partial y}\left(\frac{1}{2 \lambda} D^{\prime}(u)\left(\left(u_{x}\right)^{2}-\left(u_{y}\right)^{2}\right)-\frac{1}{\lambda}\left(\frac{\partial}{\partial x}\left(D(u) u_{x}\right)-\frac{\partial}{\partial y}\left(D(u) u_{y}\right)\right)\right)\right] \\
& +\mathrm{q}_{3} \frac{L}{2}\left(\frac{\partial}{\partial x},-\frac{\partial}{\partial y}\right) \cdot\left[\frac{1}{\lambda} \nabla \sigma^{(1)}-D^{\prime}(u) \sigma^{(1)} \nabla u\right] \text {, } \\
& \mathcal{D}^{3} \mathcal{C}\left(\mathbf{n}^{(0)}\right) \cdot \mathbf{n}^{(1)} \mathbf{n}^{(1)} \mathbf{n}^{(1)}
\end{aligned}
$$




$$
\begin{aligned}
= & \mathbf{q}_{1} \frac{24 L}{\lambda^{3}}\left(u_{x}\right)\left(\left(\lambda \sigma^{(1)}\right)^{2}-\left(L u_{y}\right)^{2}\right)+\mathbf{q}_{2} \frac{24 L}{\lambda^{3}}\left(u_{y}\right)\left(\left(\lambda \sigma^{(1)}\right)^{2}-\left(L u_{x}\right)^{2}\right) \\
- & \mathbf{q}_{3} \frac{12 L^{2}}{\lambda^{2}} \sigma^{(1)}\left(\left(u_{x}\right)^{2}-\left(u_{y}\right)^{2}\right), \\
\mathcal{D}^{2} \mathcal{C}\left(\mathbf{n}^{(0)}\right) \cdot \dot{\mathbf{n}}^{(1)} \mathbf{n}^{(2)} & \\
= & -\mathbf{q}_{1} \frac{L}{4} D^{\prime}(u)\left[\left(2 \lambda \sigma^{(1)}\right)^{2} D^{\prime}(u) u_{x}-4 \lambda\left(\sigma^{(2)} u_{x}+\sigma^{(1)} \sigma_{x}^{(1)}\right)\right. \\
& \left.+L^{2} u_{x}\left(D^{\prime}(u)\left(\left(u_{x}\right)^{2}-\left(u_{y}\right)^{2}\right)-2\left(\frac{\partial}{\partial x}\left(D(u) u_{x}\right)-\frac{\partial}{\partial y}\left(D(u) u_{y}\right)\right)\right)\right] \\
- & \mathbf{q}_{2} \frac{L}{4} D^{\prime}(u)\left[\left(2 \lambda \sigma^{(1)}\right)^{2} D^{\prime}(u) u_{y}-4 \lambda\left(\sigma^{(2)} u_{y}+\sigma^{(1)} \sigma_{y}^{(1)}\right)\right. \\
& \left.\quad-L^{2} u_{y}\left(D^{\prime}(u)\left(\left(u_{x}\right)^{2}-\left(u_{y}\right)^{2}\right)-2\left(\frac{\partial}{\partial x}\left(D(u) u_{x}\right)-\frac{\partial}{\partial y}\left(D(u) u_{y}\right)\right)\right)\right] \\
+ & \mathbf{q}_{3} \frac{L^{2}}{4} D^{\prime}(u)\left[3 \lambda \sigma^{(1)} D^{\prime}(u)\left(\left(u_{x}\right)^{2}-\left(u_{y}\right)^{2}\right)-2\left(\sigma_{x}^{(1)} u_{x}+\sigma_{y}^{(1)} u_{y}\right)\right. \\
& \left.-2 \lambda \sigma^{(1)}\left(\frac{\partial}{\partial x}\left(D(u) u_{x}\right)-\frac{\partial}{\partial y}\left(D(u) u_{y}\right)\right)\right] .
\end{aligned}
$$

$\mathcal{O}\left[\delta^{4}\right]$

Since $\sigma^{(1)}(x, y ; t)=0$ satisfies the $\mathcal{O}\left[\delta^{3}\right]$ consistency condition (Eq. 2.31), we have made this assumption in the derivation of the $\mathcal{O}\left[\delta^{4}\right]$ terms.

$$
\begin{aligned}
& {\left[\left(\vec{e}_{k} \cdot \nabla\right)^{4} n_{k}^{(0)}\right]=\mathrm{q}_{0} \frac{1}{2}\left(u_{x x x x}+u_{y y y y}\right)+\mathrm{q}_{3} \frac{1}{2}\left(u_{x x x x}-u_{y y y y}\right),} \\
& {\left[\left(\vec{e}_{k} \cdot \nabla\right)^{2} \frac{\partial}{\partial t} n_{k}^{(0)}\right]=\mathrm{q}_{0} \frac{1}{2}\left(u_{x x t}+u_{y y t}\right)+\mathrm{q}_{3} \frac{1}{2}\left(u_{x x t}-u_{y y t}\right),} \\
& \frac{\partial^{2}}{\partial t^{2}} \mathrm{n}^{(0)}=\mathrm{q}_{0} u_{t t}, \\
& {\left[\left(\vec{e}_{k} \cdot \nabla\right) n_{k}^{(3)}\right]=\mathrm{q}_{0} \frac{1}{2}\left(\frac{\partial}{\partial x} c_{1}^{(3)}+\frac{\partial}{\partial y} c_{2}^{(3)}\right)+\mathrm{q}_{1} \sigma_{x}^{(3)}+\mathrm{q}_{2} \sigma_{y}^{(3)}+\mathrm{q}_{3} \frac{1}{2}\left(\frac{\partial}{\partial x} c_{1}^{(3)}-\frac{\partial}{\partial y} c_{2}^{(3)}\right),} \\
& {\left[\left(\vec{e}_{k} \cdot \nabla\right)^{2} n_{k}^{(2)}\right]}
\end{aligned}
$$




$$
\begin{aligned}
& =\mathbf{q}_{0} \frac{1}{2}\left[\sigma_{x x}^{(2)}+\sigma_{y y}^{(2)}+\left(\frac{\partial^{2}}{\partial x^{2}}-\frac{\partial^{2}}{\partial y^{2}}\right) c_{3}^{(2)}\right]+\mathbf{q}_{3} \frac{1}{2}\left[\sigma_{x x}^{(2)}-\sigma_{y y}^{(2)}+\left(\frac{\partial^{2}}{\partial x^{2}}+\frac{\partial^{2}}{\partial y^{2}}\right) c_{3}^{(2)}\right], \\
& \frac{\partial}{\partial t} n^{(2)}=q_{0} \sigma_{t}^{(2)}+q_{3} \frac{\partial}{\partial t} c_{3}^{(2)} \text {, } \\
& {\left[\left(\vec{e}_{k} \cdot \nabla\right)^{3} n_{k}^{(1)}\right]} \\
& =\mathrm{q}_{0} \frac{L}{2}\left(\frac{\partial^{3}}{\partial x^{3}}, \frac{\partial^{3}}{\partial y^{3}}\right) \cdot\left(\frac{1}{\lambda} \nabla u\right)+\mathrm{q}_{3} \frac{L}{2}\left(\frac{\partial^{3}}{\partial x^{3}},-\frac{\partial^{3}}{\partial y^{3}}\right) \cdot\left(\frac{1}{\lambda} \nabla u\right) \\
& {\left[\left(\vec{e}_{k} \cdot \nabla\right) \frac{\partial}{\partial t} n_{k}^{(1)}\right]} \\
& =\mathrm{q}_{0} \frac{L}{2} \nabla \cdot \frac{\partial}{\partial t}\left(\frac{1}{\lambda} \nabla u\right)+\mathrm{q}_{3} \frac{L}{2}\left(\frac{\partial}{\partial x},-\frac{\partial}{\partial y}\right) \cdot \frac{\partial}{\partial t}\left(\frac{1}{\lambda} \nabla u\right) \\
& \mathcal{D}^{4} \mathcal{C}\left(n^{(0)}\right) \cdot n^{(1)} n^{(1)} n^{(1)} n^{(1)}=0, \\
& \mathcal{D}^{2} \mathcal{C}\left(\mathbf{n}^{(0)}\right) \cdot \mathbf{n}^{(2)} \mathbf{n}^{(2)} \\
& =\mathrm{q}_{3} \frac{L^{2}}{2} D^{\prime}(u) \lambda \sigma^{(2)}\left[\frac{1}{2}\left(\left(u_{x}\right)^{2}-\left(u_{y}\right)^{2}\right)-\left(\frac{\partial}{\partial x},-\frac{\partial}{\partial y}\right) \cdot D(u) \nabla u\right] \text {, } \\
& \mathcal{D}^{3} \mathcal{C}\left(\mathbf{n}^{(0)}\right) \cdot \mathbf{n}^{(1)} \mathbf{n}^{(1)} n^{(2)} \\
& =\mathrm{q}_{3} \frac{2 L^{2}}{\lambda^{3}}\left[\frac{L^{2}}{2} D^{\prime}(u)\left(\left(u_{x}\right)^{4}-\left(u_{y}\right)^{4}\right)\right. \\
& \left.-L^{2}\left(\left(u_{x}\right)^{2}+\left(u_{y}\right)^{2}\right)\left(\frac{\partial}{\partial x},-\frac{\partial}{\partial y}\right) \cdot D(u) \nabla u-2 \lambda \sigma^{(2)}\left(\left(u_{x}\right)^{2}-\left(u_{y}\right)^{2}\right)\right] \text {, } \\
& \mathcal{D}^{2} \mathcal{C}\left(\mathbf{n}^{(0)}\right) \cdot \mathbf{n}^{(1)} \mathbf{n}^{(3)} \\
& =\mathrm{q}_{1} L D^{\prime}(u) \lambda \sigma^{(3)} u_{x}+\mathrm{q}_{2} L D^{\prime}(u) \lambda \sigma^{(3)} u_{y} \\
& \text { - } \mathrm{q}_{3} \frac{L}{2} D^{\prime}(u) \lambda\left(c_{1}^{(3)} u_{x}-c_{2}^{(3)} u_{y}\right) \text {. }
\end{aligned}
$$

$\mathcal{O}\left[\delta^{5}\right]$

$$
\left[\left(\vec{e}_{k} \cdot \nabla\right)^{5} n_{k}^{(0)}\right]=q_{1} \frac{\partial^{5}}{\partial x^{5}} u+q_{2} \frac{\partial^{5}}{\partial y^{5}} u
$$


APPENDIX A. LB ANALYSIS DETAILS

$230 / 240$

$$
\begin{aligned}
& \begin{array}{l}
{\left[\left(\vec{e}_{k} \cdot \nabla\right) \frac{\partial^{2}}{\partial t^{2}} n_{k}^{(0)}\right]=\mathrm{q}_{1} u_{x t t}+\mathrm{q}_{2} u_{y t t},} \\
{\left[\left(\vec{e}_{k} \cdot \nabla\right)^{3} \frac{\partial}{\partial t} n_{k}^{(0)}\right]=\mathrm{q}_{1} u_{x x x t}+\mathrm{q}_{2} u_{y y y t},}
\end{array} \\
& {\left[\left(\vec{e}_{k} \cdot \nabla\right)^{4} n_{k}^{(1)}\right]=\mathrm{q}_{1} L \frac{\partial^{4}}{\partial x^{4}}\left(\frac{1}{\lambda} u_{x}\right)+\mathbf{q}_{2} L \frac{\partial^{4}}{\partial y^{4}}\left(\frac{1}{\lambda} u_{y}\right),} \\
& {\left[\left(\vec{e}_{k} \cdot \nabla\right)^{2} \frac{\partial}{\partial t} n_{k}^{(1)}\right]=\mathrm{q}_{1} L \frac{\partial^{3}}{\partial x^{2} \partial t}\left(\frac{1}{\lambda} u_{x}\right)+\mathrm{q}_{2} L \frac{\partial^{3}}{\partial y^{2} \partial t}\left(\frac{1}{\lambda} u_{y}\right),} \\
& \frac{\partial^{2}}{\partial t^{2}} n_{k}^{(1)}=\mathbf{q}_{1} L \frac{\partial^{2}}{\partial t^{2}}\left(\frac{1}{\lambda} u_{x}\right)+\mathbf{q}_{2} L \frac{\partial^{2}}{\partial t^{2}}\left(\frac{1}{\lambda} u_{y}\right), \\
& {\left[\left(\vec{e}_{k} \cdot \nabla\right)^{3} n_{k}^{(2)}\right]=\mathrm{q}_{1} \frac{\partial^{3}}{\partial x^{3}}\left(\sigma^{(2)}+c_{3}^{(2)}\right)+\mathrm{q}_{2} \frac{\partial^{3}}{\partial y^{3}}\left(\sigma^{(2)}+c_{3}^{(2)}\right),} \\
& {\left[\left(\vec{e}_{k} \cdot \nabla\right) \frac{\partial}{\partial t} n_{k}^{(2)}\right]=\mathbf{q}_{1} \frac{\partial^{2}}{\partial x \partial t}\left(\sigma^{(2)}+c_{3}^{(2)}\right)+\mathbf{q}_{2} \frac{\partial^{2}}{\partial y \partial t}\left(\sigma^{(2)}-c_{3}^{(2)}\right),} \\
& {\left[\left(\vec{e}_{k} \cdot \nabla\right)^{2} n_{k}^{(3)}\right]=\mathbf{q}_{0} \frac{1}{2}\left(\sigma_{x x}^{(3)}+\sigma_{y y}^{(3)}\right)+\mathbf{q}_{1} \frac{\partial^{2}}{\partial x^{2}} c_{1}^{(3)}+\mathbf{q}_{2} \frac{\partial^{2}}{\partial y^{2}} c_{2}^{(3)}+\mathbf{q}_{3} \frac{1}{2}\left(\sigma_{x x}^{(3)}-\sigma_{y y}^{(3)}\right),} \\
& \frac{\partial}{\partial t} n_{k}^{(3)}=\mathbf{q}_{0} \sigma_{t}^{(3)}+\mathbf{q}_{1} \frac{\partial}{\partial t} c_{1}^{(3)}+\mathbf{q}_{2} \frac{\partial}{\partial t} c_{2}^{(3)} \text {, } \\
& {\left[\left(\vec{e}_{k} \cdot \nabla\right) n_{k}^{(4)}\right]} \\
& =\mathrm{q}_{0} \frac{L}{2} \nabla \cdot\left(\frac{1}{\lambda} \nabla \sigma^{(3)}-D^{\prime}(u) \sigma^{(3)} \nabla u\right) \\
& +\mathrm{q}_{1} \frac{\partial}{\partial x}\left(\sigma^{(4)}+c_{3}^{(4)}\right)+\mathrm{q}_{2} \frac{\partial}{\partial y}\left(\sigma^{(4)}-c_{3}^{(4)}\right) \\
& +\mathrm{q}_{3} \frac{L}{2}\left(\frac{\partial}{\partial x},-\frac{\partial}{\partial y}\right) \cdot\left[\frac{1}{\lambda} \nabla \sigma^{(3)}-D^{\prime}(u) \sigma^{(3)} \nabla u\right] \text {. }
\end{aligned}
$$




\section{Appendix B $\mathrm{LB}_{2}$ Analysis Details}

This appendix lists some of the details regarding the discrete Chapman-Enskog analysis of the lattice Boltzmann method, $\mathrm{LB}_{2}$. The listing is order by order as in Section 3.1.5. We denote zero order parameter $\sigma^{(0)}=u$, and also use

$$
\begin{aligned}
D(u) & =-\frac{1}{\lambda}-\frac{1}{2} \\
D^{\prime}(u) & =\frac{4 u}{\lambda^{2}},
\end{aligned}
$$

in which $\lambda=-2(1-u)(1+u)$. Also, regarding notation, at and beyond $\mathcal{O}\left[\delta^{4}\right]$, some of the $c_{k}^{(j)}$ and $\tilde{c}_{k}^{(j)}$ coefficients as defined in Section 3.1 .5 are used; otherwise, the expressions are undescriptively long.

One further note is that since the $\mathrm{LB}_{2} \mathrm{n}^{(0)}$ and $\mathrm{n}^{(1)}$ terms are symbolically identical to the $\mathrm{LB}_{1} \mathrm{n}^{(0)}$ and $\mathrm{n}^{(1)}$ terms, only those advection expansion-related quantities involving higher orders in $\mathrm{n}$ are listed. For those quantities not listed here, refer to Appendix $\mathrm{A}$, with $\lambda, D(u)$, and $D^{\prime}(u)$ defined as above, and $c_{k}^{(j)}$ clefined in the discrete Chapman-Enskog procedure applied to $\mathrm{n}$ of $\mathrm{LB}_{2}$ (Section 3.1.5). $\mathcal{O}\left[\delta^{2}\right]:$

$$
\mathcal{D}^{2} \mathcal{C}\left(n^{(0)}\right) \cdot n^{(1)} n^{(1)}
$$




$$
=\mathrm{q}_{1} 2 L \lambda \sigma^{(1)} D^{\prime}(u) u_{x}+\mathrm{q}_{2} 2 L \lambda \sigma^{(1)} D^{\prime}(u) u_{y}-\mathrm{q}_{3} \frac{2 L^{2}(2 u-1)}{\lambda^{2}}\left(\left(u_{x}\right)^{2}-\left(u_{y}\right)^{2}\right) .
$$

() $\left[\delta^{3}\right]:$

$$
\begin{aligned}
& {\left[\left(\vec{e}_{k} \cdot \nabla\right) n_{k}^{(2)}\right]} \\
& =\mathrm{q}_{0} \frac{L}{2} \nabla \cdot\left[\frac{1}{\lambda} \nabla \sigma^{(1)}-D^{\prime}(u) \sigma^{(1)} \nabla u\right] \\
& +\mathrm{q}_{1}\left[\sigma_{x}^{(2)}+L^{2} \frac{\partial}{\partial x}\left(\frac{1}{2 \lambda_{3}}\left(\frac{\partial}{\partial x},-\frac{\partial}{\partial y}\right) \cdot D(u) \nabla u+\frac{2 u-1}{\lambda_{3} \lambda^{2}}\left(\left(u_{x}\right)^{2}-\left(u_{y}\right)^{2}\right)\right)\right] \\
& +\mathrm{q}_{2}\left[\sigma_{y}^{(2)}+L^{2} \frac{\partial}{\partial y}\left(\frac{1}{2 \lambda_{3}}\left(\frac{\partial}{\partial x},-\frac{\partial}{\partial y}\right) \cdot D(u) \nabla u-\frac{2 u-1}{\lambda_{3} \lambda^{2}}\left(\left(u_{x}\right)^{2}-\left(u_{y}\right)^{2}\right)\right)\right] \\
& +\mathrm{q}_{3} \frac{L}{2}\left(\frac{\partial}{\partial x}, \frac{\partial}{\partial y}\right) \cdot\left(\frac{1}{\lambda} \nabla \sigma^{(1)}-D^{\prime}(u) \sigma^{(1)} \nabla u\right) \\
& \mathcal{D}^{3} \mathcal{C}\left(\mathbf{n}^{(0)}\right) \cdot \mathbf{n}^{(1)} \mathbf{n}^{(1)} \mathbf{n}^{(1)} \\
& =\mathrm{q}_{1} \frac{12 L}{\lambda^{3}} u_{x}\left(\left(\lambda \sigma^{(1)}\right)^{2}-\left(L u_{y}\right)^{2}\right)+\mathrm{q}_{2} \frac{12 L}{\lambda^{3}} u_{y}\left(\left(\lambda \sigma^{(1)}\right)^{2}-\left(L u_{x}\right)^{2}\right) \\
& \text { - } \mathrm{q}_{3} \frac{12 L^{2}}{\lambda^{2}} \sigma^{(1)}\left(\left(u_{x}\right)^{2}-\left(u_{y}\right)^{2}\right) \text {, } \\
& \mathcal{D}^{2} \mathcal{C}\left(\mathbf{n}^{(0)}\right) \cdot \mathbf{n}^{(1)} \mathbf{n}^{(2)} \\
& =\mathrm{q}_{1} \frac{L}{4} D^{\prime}(u)\left[2 \lambda\left(\sigma^{(2)} u_{x}+\sigma^{(1)} \sigma_{x}^{(1)}\right)-2\left(\lambda \sigma^{(2)}\right)^{2} D^{\prime}(u) u_{x}\right. \\
& \left.+L^{2} u_{x}\left(\frac{\partial}{\partial x},-\frac{\partial}{\partial y}\right) \cdot D(u) \nabla u-\frac{2 L^{2}(2 u-1)}{\lambda} u_{x}\left(\left(u_{x}\right)^{2}-\left(u_{y}\right)^{2}\right)\right] \\
& +\mathrm{q}_{2} \frac{L}{2 \lambda}\left[2 \lambda\left(\sigma^{(2)} u_{y}+\sigma^{(1)} \sigma_{y}^{(1)}\right)-2\left(\lambda \sigma^{(2)}\right)^{2} D^{\prime}(u) u_{y}\right. \\
& \left.-L^{2} u_{y}\left(\frac{\partial}{\partial x},-\frac{\partial}{\partial y}\right) \cdot D(u) \nabla u+\frac{2 L^{2}(2 u-1)}{\lambda} u_{y}\left(\left(u_{x}\right)^{2}-\left(u_{y}\right)^{2}\right)\right] \\
& +\mathrm{q}_{3} \frac{2 L^{2}(2 u-1)}{\lambda^{2}}\left[\sigma^{(1)}\left((2 u-1)+D^{\prime}(u) \lambda\right)\left(\left(u_{x}\right)^{2}-\left(u_{y}\right)^{2}\right)\right. \\
& \left.-\lambda \sigma^{(1)}\left(\frac{\partial}{\partial x},-\frac{\partial}{\partial y}\right) \cdot D(u) \nabla u-\left(\sigma_{x}^{(1)} u_{x}-\sigma_{y}^{(1)} u_{y}\right)\right] .
\end{aligned}
$$

$\mathcal{O}\left[\delta^{4}\right]:$ 
Recalling that $\sigma^{(1)}(x, y ; t)=$ const, satisfies the $\mathcal{O}\left[\delta^{3}\right]$ consistency condition, i,e., Eq. 3.8 , the expressions below assume $\sigma^{(1)}(x, y ; t)=0$.

$$
\begin{aligned}
& {\left[\left(\vec{e}_{k} \cdot \nabla\right)^{2} n_{k}^{(2)}\right]} \\
& =\mathrm{q}_{0} \frac{1}{2}\left[\sigma_{x x}^{(2)}+\sigma_{y y}^{(2)}+\frac{\partial^{2}}{\partial x^{2}} c_{3}^{(2)}-\frac{\partial^{2}}{\partial y^{2}} c_{3}^{(2)}\right]+\mathrm{q}_{3} \frac{1}{2}\left[\sigma_{x x}^{(2)}-\sigma_{y y}^{(2)}+\frac{\partial^{2}}{\partial x^{2}} c_{3}^{(2)}+\frac{\partial^{2}}{\partial y^{2}} c_{3}^{(2)}\right], \\
& \frac{\partial}{\partial t} \mathbf{n}^{(2)}=q_{0} \sigma_{t}^{(2)}+\mathbf{q}_{3} \frac{\partial}{\partial t} c_{3}^{(2)}, \\
& {\left[\left(\vec{e}_{k}, \nabla\right) n_{k}^{(3)}\right]} \\
& =\mathrm{q}_{0} \frac{1}{2}\left(\frac{\partial}{\partial x} c_{1}^{(3)}+\frac{\partial}{\partial y} c_{2}^{(3)}\right)+\mathrm{q}_{1} \sigma_{x}^{(3)}+\mathrm{q}_{2} \sigma_{y}^{(3)}+\mathrm{q}_{3} \frac{1}{2}\left(\frac{\partial}{\partial x} c_{1}^{(3)}-\frac{\partial}{\partial y} c_{2}^{(3)}\right), \\
& \mathcal{D}^{4} \mathcal{C}\left(\mathbf{n}^{(0)}\right) \cdot \mathbf{n}^{(1)} \mathrm{n}^{(1)} \mathbf{n}^{(1)} \mathbf{n}^{(1)}=0 \\
& \mathcal{D}^{3} \mathcal{C}\left(\mathbf{n}^{(0)}\right) \cdot \mathbf{n}^{(1)} \mathbf{n}^{(1)} \mathbf{n}^{(2)} \\
& =\mathrm{q}_{3} \frac{2 L^{2}}{\lambda^{3}}\left[\frac{2 L^{2}(2 u-1)}{\lambda \lambda_{3}}\left(\left(u_{x}\right)^{4}-\left(u_{y}\right)^{4}\right)\right. \\
& \left.-\frac{L^{2} \lambda}{\lambda_{3}}\left(\left(u_{x}\right)^{2}+\left(u_{y}\right)^{2}\right)\left(\frac{\partial}{\partial x},-\frac{\partial}{\partial y}\right) \cdot D(u) \nabla u-2 \lambda \sigma^{(2)}\left(\left(u_{x}\right)^{2}-\left(u_{y}\right)^{2}\right)\right], \\
& \mathcal{D}^{2} \mathcal{C}\left(\mathbf{n}^{(0)}\right) \cdot \mathbf{n}^{(2)} \mathbf{n}^{(2)} \\
& =\mathrm{q}_{3} \frac{4 L^{2}(2 u-1)}{\lambda_{3}} \sigma^{(2)}\left[\frac{2(2 u-1)}{\lambda^{2}}\left(\left(u_{x}\right)^{2}-\left(u_{y}\right)^{2}\right)-\left(\frac{\partial}{\partial x},-\frac{\partial}{\partial y}\right) \cdot D(u) \nabla u\right] \text {, } \\
& \mathcal{D}^{2} \mathcal{C}\left(\mathrm{n}^{(0)}\right) \cdot \mathrm{n}^{(1)} \mathrm{n}^{(3)} \\
& =\mathrm{q}_{1} L D^{\prime}(u) \lambda \sigma^{(3)} u_{x}+\mathrm{q}_{2} L D^{\prime}(u) \lambda \sigma^{(3)} u_{\mathrm{y}} \\
& \text { - } \mathrm{q}_{3} \frac{2 L(2 u-1)}{\lambda}\left(c_{1}^{(3)} u_{x}-c_{2}^{(3)} u_{y}\right) \text {. } \\
& {\left[\left(\vec{e}_{k} \cdot \nabla\right)^{3} n_{k}^{(2)}\right]=\mathrm{q}_{1} \frac{\partial^{3}}{\partial x^{3}}\left(\sigma^{(2)}+c_{3}^{(2)}\right)+\mathrm{q}_{2} \frac{\partial^{3}}{\partial y^{3}}\left(\sigma^{(2)}+c_{3}^{(2)}\right),}
\end{aligned}
$$


214

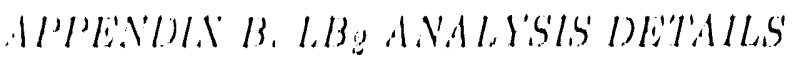

$$
\begin{aligned}
& {\left[\left(\vec{c}_{k} \cdot \nabla\right) \frac{\partial}{\partial t} n_{k}^{(2)}\right]=q_{1} \frac{\partial^{2}}{\partial x \partial t}\left(\sigma^{(2)}+\left(c_{3}^{(2)}\right)+q_{2} \frac{i)^{2}}{\partial y(j)}\left(\sigma^{(2)}-c_{i}^{(2)}\right),\right.} \\
& {\left[\left(\vec{e}_{k} \cdot \Gamma\right)^{2} u_{k}^{(3)}\right]=\mathrm{q}_{0} \frac{1}{2}\left(\sigma_{x x}^{(3)}+\sigma_{y y}^{(3)}\right)+\mathrm{q}_{1} \frac{\partial^{2}}{\partial x^{2}} c_{1}^{(3)}+\mathrm{q}_{2} \frac{\partial^{2}}{\partial y^{2} c^{2}} c_{z}^{(3)}+\mathrm{q}_{3} \frac{1}{2}\left(\sigma_{x^{\prime} x}^{(3)}-\sigma_{y y}^{(3)}\right),} \\
& \frac{\partial}{\partial t} n_{k}^{(3)}=\mathrm{q}_{0} \sigma_{t}^{(3)}+\mathrm{q}_{1} \frac{\partial}{\partial t} c_{1}^{(3)}+\mathrm{q}_{2} \frac{\partial}{\partial t} c_{2}^{(3)}, \\
& {\left[\left(\vec{e}_{k} \cdot \nabla\right) n_{k}^{(4)}\right]} \\
& =\mathrm{q}_{0} \frac{L}{2} \nabla \cdot\left(\frac{1}{\lambda} \nabla \sigma^{(3)}-D^{\prime}(u) \sigma^{(3)} \nabla u\right) \\
& +\mathrm{q}_{1} \frac{\partial}{\partial x}\left(\sigma^{(4)}+c_{3}^{(4)}\right)+\mathrm{q}_{2} \frac{\partial}{\partial y}\left(\sigma^{(4)}-c_{3}^{(4)}\right) \\
& +\mathrm{q}_{3} \frac{L}{2}\left(\frac{\partial}{\partial x},-\frac{\partial}{\partial y}\right) \cdot \nabla\left[\frac{1}{\lambda} \nabla \sigma^{(3)}-D^{\prime}(u) \sigma^{(3)} \nabla u\right] \text {. }
\end{aligned}
$$




\section{Appendix C}

\section{Convergence of a Nonlinear Finite Difference Scheme}

This appendix presents a proof of convergence of an explicit conservative monotone finite difference method for a nonlinear advection-diffusion equation, namely, the one-dimensional viscous Burgers equation. This converget ice proof serves as a model for convergence proofs of lattice gas methods. First, we give the method, then we prove convergence via proving consistency, establishing a maximum principle of the difference scheme, and proving stability. Stability is obtained directly from the (nonlinear) operator; it is not first linearized. See, for exanple, [69, Ch. IV] for further information regarding convergence of monotone finite difference methods.

\section{C.1 Nonlinear Problem}

Let $u(x, t)$ satisfy the one.dimensional viscous Burgers' equation,

$$
\frac{\partial}{\partial t} u(x, t)+u(x, t) \frac{\partial}{\partial x} u(x, t)=u \cdot \frac{\partial^{2}}{\partial x^{2}} u(u, t) \quad \text { for }(u, t) \in[0, L] \times\left[0, I^{\prime}\right]
$$


with periodic boundary conditions and smooth initial condition $u(x, 0)=u^{i}(x)$ for some positive constants $L$ and T. Then Eq. C.1 in conservative form is

$$
g(u(x, t)) \equiv \frac{\partial}{\partial t} u+\frac{\partial}{\partial x} F=0
$$

with

$$
F=\frac{1}{2} u^{2}-\nu \frac{\partial}{\partial x} u
$$

\section{C.2 Explicit Finite Difference Method}

Let the conservative finite difference method be

$$
\begin{aligned}
& G\left(U_{j}^{n}\right) \equiv \frac{U_{j}^{n+1}-U_{j}^{n}}{\Delta t}+\frac{F_{j+1 / 2}^{n+1 / 2}-F_{j-1 / 2}^{n+1 / 2}}{\Delta x}=0, \\
& F_{j+1 / 2}^{n+1 / 2} \equiv \frac{\left(U_{j+1}^{n}\right)^{2}+\left(U_{j}^{n}\right)^{2}}{4}-\nu \frac{U_{j+1}^{n}-U_{j}^{n}}{\Delta x},
\end{aligned}
$$

where $U_{j}^{n}$ is understood as an approximation to $A u(j \Delta x, n \Delta t)$, and averaging operator $A$ is defined by

$$
A u(x, t) \equiv \int_{x-\Delta x / 2}^{x+\Delta x / 2} u(x, t) d x
$$

\section{C.3 Maximum Principle}

We need two maximum principles for stability, one for $g(u)$ and one for $G(U)$. These may be stated as:

Lemma C.1 (Continuum Maximum Principle). Given

$$
u: \Omega=[0, L] \times(0, T] \rightarrow \mathcal{R}
$$

satisfying Eq. C.2 with " > 0, periodic boundary conditions and smooth, bounded initial condition $u(x ; 0)=u_{I}(x)$. Then the maximur, and minimum values of $\rho(x ; t)$ occur at the initial boundary. 
Proof. See Lemma 3.12 and its proof, which is based on general theoretical results for maximum and ninimum principles for nonlinear parabolic partial differential equations.

Lemma C.2 (Discre'te Maximum Principle). Let $n$ be given, and let $U_{j}^{n}$ be determined $\forall j$ from the difference method, Eq. C.3. Suppose $\Delta t \leq(\Delta x)^{2} /(2 \nu)$ and $\left\|U^{0}\right\|_{\infty} \leq 2 \nu / d x$. Then $\left\|U^{n}\right\|_{\infty} \leq\left\|U^{0}\right\|_{\infty}$.

Proof. First, write the difference equation as

$$
\begin{aligned}
U_{j}^{n+1} & =U_{j}^{n}-\frac{\Delta t}{4 \Delta x}\left[\left(U_{j+1}^{n}\right)^{2}-\left(U_{j-1}^{n}\right)^{2}\right]+\frac{\nu \Delta t}{(\Delta x)^{2}}\left[U_{j+1}^{n}-2 U_{j}^{n}+U_{j-1}^{n}\right](\text { C.4 }) \\
& \equiv H\left(U_{j+1}^{n}, U_{j}^{n}, U_{j-1}^{n}\right) .
\end{aligned}
$$

Note that

$$
\begin{aligned}
& \frac{\partial}{\partial U_{j}^{n}} H\left(U_{j+1}^{n}, U_{j}^{n}, U_{j-1}^{n}\right)=0 \\
& \frac{\partial}{\partial U_{j \pm 1}^{n}} H\left(U_{j+1}^{n}, U_{j}^{n}, U_{j-1}^{n}\right)=\frac{\Delta t}{2 \Delta x}\left(\frac{2 \nu}{\Delta x} \mp U_{j \pm 1}^{n}\right) \geq 0,
\end{aligned}
$$

if the monotonicity condition, $\left\|U^{n}\right\|_{\infty} \leq 2 \nu / \Delta x$, holds. Then let $M_{n}=\left\|U^{n}\right\|_{\infty}$ and assume $2 \nu \Delta t /(\Delta x)^{2} \leq 1$ (the stability criterion) to get

$$
\begin{aligned}
& U_{j}^{n+1} \leq U_{j}^{n}+\frac{2 \nu \Delta t}{(\Delta x)^{2}}\left(M_{n}-U_{j}^{n}\right) \leq+M_{n}, \\
& U_{j}^{n+1} \geq U_{j}^{n}-\frac{2 \nu \Delta t}{(\Delta x)^{2}}\left(M_{n}+U_{j}^{n}\right) \geq-M_{n},
\end{aligned}
$$

i.e., $\left\|U^{n+1}\right\|_{\infty} \leq M_{n}=\left\|U^{n}\right\|_{\infty}$.

Now, to get $\left\|U^{n+1}\right\|_{\infty} \leq\left\|U^{0}\right\|_{\infty}$ we use induction on $n$. The base step follows from the preceding argument with $n=1$, assuming the the monotonicity condition is initially satisfied, i.e., $\left\|U^{0}\right\|_{\infty} \leq 2 \nu / \Delta x$. For the induction step assume that $\left\|U^{n}\right\|_{\infty} \leq$ $\left\|U^{0}\right\|_{\infty}$. Then also from the preceding argument, it follows that $\left\|U^{n+1}\right\|_{\infty} \leq\left\|U^{n}\right\|_{\infty}$. Thus, $\left\|U^{n+1}\right\|_{\infty} \leq\left\|U^{0}\right\|_{\infty}$. 


\section{C.4 Consistency}

Sufficient to prove consistency is to show $\lim _{\Delta x, \Delta t \rightarrow \infty}\|G(A u)-A g(u)\|_{\infty}=0$ for all $x$ and $t$. Let $V \equiv A u$. Then

$$
\begin{aligned}
G(A u) & =\frac{V_{j}^{n+1}-V_{j}^{n}}{\Delta t}+\frac{1}{4 \Delta x}\left[\left(V_{j+1}^{n}\right)^{2}-\left(V_{j-1}^{n}\right)^{2}\right]-\frac{\nu}{(\Delta x)^{2}}\left[V_{j+1}^{n}-2 V_{j}^{n}+V_{j-1}^{n}\right] \\
& =\frac{\partial}{\partial t} V(x, t)+\frac{\partial}{\partial x}\left(\frac{V^{2}(x, t)}{2}\right)-\nu \frac{\partial^{2}}{\partial x^{2}} V(x, t)+\mathcal{O}[\Delta t]+\mathcal{O}\left[(\Delta x)^{2}\right] .
\end{aligned}
$$

And noting that ${ }^{1}$

$$
\frac{\partial}{\partial x} V(x, t)=\frac{\partial}{\partial x}\left[\frac{1}{\Delta x} \int_{x-\Delta x / 2}^{x+\Delta x / 2} u(x, t) d x\right]=A \frac{\partial}{\partial x} u(x, t),
$$

it follows that

$$
\begin{aligned}
A g(u) & =A\left(\frac{\partial}{\partial t} u(x, t)\right)+A\left(\frac{\partial}{\partial x} \frac{u^{2}(x, t)}{2}\right)-\nu A\left(\frac{\partial^{2}}{\partial x^{2}} u(x, t)\right) \\
& =\frac{\partial}{\partial t} V(x, t)+\frac{u^{2}(x+\Delta x / 2, t)-u^{2}}{2 \Delta x} \frac{(x-\Delta x / 2, t)}{2}-\nu \frac{\partial^{2}}{\partial x^{2}} V(x, t) .
\end{aligned}
$$

Thus,

$$
\begin{aligned}
G(A u)-A g(u) & =\frac{\partial}{\partial x}\left(\frac{V^{2}(x, t)}{2}\right)-\frac{u^{2}(x+\Delta x / 2, t)-u^{2}(x-\Delta x / 2, t)}{2 \Delta x} \\
& +\mathcal{O}[\Delta t]+\mathcal{O}\left[(\Delta x)^{2}\right] .
\end{aligned}
$$

Now,

$$
\begin{aligned}
\frac{\partial}{\partial x}\left[\frac{V^{2}(x, t)}{2}\right] & =V(x, t) \frac{\partial}{\partial x} V(x, t) \\
& =\left[\frac{u(x+\Delta x / 2, t)+u(x-\Delta x / 2, t)}{2}+\mathcal{O}\left[(\Delta x)^{3}\right]\right] \\
& {\left[\frac{u(x+\Delta x / 2, t)-u(x-\Delta x / 2, t)}{\Delta x}+\mathcal{O}\left[(\Delta x)^{2}\right]\right] } \\
& =\frac{u^{2}(x+\Delta x / 2, t)-u^{2}(x-\Delta x / 2, t)}{2 \Delta x}+\mathcal{O}\left[(\Delta x)^{2}\right] .
\end{aligned}
$$

\footnotetext{
${ }^{1}$ By the Fundamental Theorem of Calculus.
} 
Hence,

$$
G(A u)-A g(u)=\mathcal{O}[\Delta t]+\mathcal{O}\left[(\Delta x)^{2}\right]
$$

goes to zero in a norm sense as $\Delta t, \Delta x \rightarrow 0$.

\section{C.5 Stability}

The consistency analysis followed directly the sort of analysis one would do for a linear problem. However, the stability analysis departs slightly from the traditional stability analysis. Instead of one linear operator for all time steps, there is a linear operator for each time step $n$. The linear operator depends on both the $n$th computed and discretized solutions.

Let $V_{j}^{n} \equiv(A u)_{j}^{n}=\left.A u\right|_{(x, t)=(j \Delta x, n \Delta t)}$. Then let $W_{j}^{n} \equiv U_{j}^{n}-V_{j}^{n}$ with

$$
U^{n} \equiv\left[W_{j}^{n}\right], \quad V^{n} \equiv\left[W_{j}^{n}\right]: \quad W^{n} \equiv\left[W_{j}^{n}\right]
$$

Then write

$$
W^{n+1}=L\left[U^{n}, V^{n}\right]\left(W^{n}\right)+\mathcal{T}\left(V^{n}\right)
$$

where linear operator $L\left[U^{n}, V^{n}\right]$ (yet to be determined) depends on $U^{n}, V^{n}, \Delta t$, and $\Delta x$, and $\mathcal{T}\left(V^{n}\right)=\mathcal{O}\left[(\Delta t)^{2}\right]+\mathcal{O}\left[\Delta t(\Delta x)^{2}\right]$ (by the consistency arguments). Then to prove stability (assuming maximum principles of both the finite difference scheme and the differential equation) is to show that $\left\|L\left[U^{n}, V^{n}\right]\right\| \leq 1$ for each $n$. Generally, this is shown through induction on $n$, where $U^{0} \equiv V^{0}$ is assumed, i.e., initial absence of error.

Noting that $\left(U_{j \pm 1}^{n}+V_{j \pm 1}^{n}\right)\left(U_{j \pm 1}^{n}-V_{j \pm 1}^{n}\right)=\left(U_{j \pm 1}^{n}\right)^{2}-\left(V_{j \pm 1}^{n}\right)^{2}$, we have

$$
W_{j}^{n+1}=W_{j}^{n}-\frac{\Delta t}{4 \Delta x}\left[\left(U_{j+1}^{n}\right)^{2}-\left(V_{j+1}^{n}\right)^{2}-\left(U_{j-1}^{n}\right)^{2}+\left(V_{j-1}^{n}\right)^{2}\right]
$$




$$
\begin{aligned}
& +\frac{\nu \Delta t}{(\Delta x)^{2}}\left[W_{j+1}^{n}-2 W_{j}^{n}+W_{j-1}^{n}\right]+\mathcal{O}\left[(\Delta t)^{2}\right]+\mathcal{O}\left[\Delta t(\Delta x)^{2}\right] \\
& =\left[\frac{\nu \Delta t}{(\Delta x)^{2}}-\frac{\Delta t}{4 \Delta x}\left(U_{j-1}^{n}+V_{j-1}^{n}\right)\right] W_{j-1}^{n}+\left[1-\frac{2 \nu \Delta t}{(\Delta x)^{2}}\right] W_{j}^{n} \\
& +\left[\frac{\nu \Delta t}{(\Delta x)^{2}}+\frac{\Delta t}{4 \Delta x}\left(U_{j+1}^{n}+V_{j+1}^{n}\right)\right] W_{j+1}^{n}+\mathcal{O}\left[(\Delta t)^{2}\right]+\mathcal{O}\left[\Delta t(\Delta x)^{2}\right]
\end{aligned}
$$

Hence,

$$
W^{n+1}=L\left[U^{n}, V^{n}\right]\left(U^{n}-V^{n}\right)+\mathcal{T}\left(V^{n}\right),^{2}
$$

where

$$
L\left[U^{n}, V^{n}\right]=\left[\begin{array}{ccccccc}
d_{0} & b_{0} & 0 & \ldots & & 0 & a_{J} \\
a_{1} & \ddots & \ddots & \ddots & & & 0 \\
0 & \ddots & & & & & \vdots \\
\vdots & \ddots & a_{j} & d_{j} & b_{j} & \ddots & \vdots \\
\vdots & & & & \ddots & 0 \\
0 & & & \ddots & \ddots & \ddots & b_{J-1} \\
b_{J} & 0 & & \ldots & 0 & a_{J} & d_{J}
\end{array}\right]
$$

in which

$$
\begin{aligned}
a_{j} & \equiv \frac{\nu \Delta t}{(\Delta x)^{2}}-\frac{\Delta t}{4 \Delta x}\left(U_{j-1}^{n}+V_{j-1}^{n}\right), \\
d_{j} & \equiv 1-\frac{2 \nu \Delta t}{(\Delta x)^{2}}, \text { and } \\
b_{j} & \equiv \frac{\nu \Delta t}{(\Delta x)^{2}}+\frac{\Delta t}{4 \Delta x}\left(U_{j+1}^{n}+V_{j+1}^{n}\right),
\end{aligned}
$$

and

$$
\mathcal{T}_{j}\left(V^{n}\right)=\mathcal{O}\left[(\Delta t)^{2}\right]+\mathcal{O}\left[\Delta t(\Delta x)^{2}\right]=\mathcal{O}\left[(\Delta x)^{4}\right]
$$

The upper right and lower left corner values of $L\left[U^{n}, V^{n}\right]$ come from the periodic boundary conditions.

Recall the 1-matrix norm, $\|\cdot\|_{1}$, on some matrix S: $\|S\|_{1}=\sup _{j} \sum_{i}\left|S_{i j}\right|$. Then

$$
\left\|L\left[U^{n}, V^{n}\right]\right\|_{1}=
$$

\footnotetext{
${ }^{2} J=L / \Delta x$ is assumed (without loss of generality) to be integral.
} 


$$
\sup _{j}\left\{\left|\frac{\nu \Delta t}{(\Delta x)^{2}}-\frac{\Delta t}{4 \Delta x}\left(U_{j}^{n}+V_{j}^{n}\right)\right|+\left|1-\frac{2 \nu \Delta t}{(\Delta x)^{2}}\right|+\left|\frac{\nu \Delta t}{(\Delta x)^{2}}+\frac{\Delta t}{4 \Delta x}\left(U_{j}^{n}+V_{j}^{n}\right)\right|\right\} .
$$

Now, the absolute value signs may be dropped if the following hold:

$$
\begin{aligned}
\frac{2 \nu \Delta t}{(\Delta x)^{2}} & \leq 1, \text { and } \\
\left\|U^{n}+V^{n}\right\|_{\infty} & \leq \frac{4 \nu}{\Delta x} .
\end{aligned}
$$

The first requirement is the usual stability criterion and is only a function of the diffusion coefficient and grid spacings. The second requirement is a monotonicity condition. It is satisfied for sufficiently small $\Delta x$. If initially satisfied, then it is always satisfied since $U$ and $V$ are initially the same $\left(U^{0}=V^{0}\right)$ and by the finite difference and continuum maximum principles. So, $\Delta x$ must be chosen to satisfy the stability criterion and the monotonicity condition $\left\|V^{0}\right\|_{\infty} \leq 2 \nu / \Delta x$. Then if these conditions are satisfied, the absolute value signs may be dropped. Some terms cancel, leaving

$$
\left\|L\left[U^{n}, V^{n}\right]\right\|_{1}=1
$$

and we have stability.

A statement regarding convergence can now be stated. We show that the finite difference method is $\mathcal{O}[\Delta t]+\mathcal{O}\left[(\Delta x)^{2}\right]$ accurate for unit time. Specifically, we show that for $n \ni 0 \leq n \Delta t \leq T$,

$$
\left\|W^{n+1}\right\|_{\ell_{1}}=\mathcal{O}\left[(\Delta x)^{2}\right]
$$

which we do by induction on $n$. First, consider the base step. We assume $W_{j}^{0}=$ $U_{j}^{0}-V_{j}^{0}=0 \forall j \in\{0,1, \ldots, J\}, J=L / \Delta x \in \mathcal{J}$. Then we have

$$
\left\|W^{1}\right\|_{\ell_{1}}=\left\|L\left[U^{1}, V^{1}\right]\left(W^{0}\right)+\mathcal{T}\left(V^{0}\right)\right\|_{\ell_{1}}=C(\Delta x)^{4}
$$


for some constant $C$. Now, consider the induction hypothesis that

$$
\left\|W^{n}\right\|_{\ell_{1}}=(n-1) C(\Delta x)^{4}
$$

We have

$$
\begin{aligned}
\left\|W^{n+1}\right\|_{\ell_{1}} & =\left\|L\left[U^{n}, V^{n}\right]\left(W^{n}\right)+\mathcal{T}\left(V^{n}\right)\right\|_{\ell_{1}} \\
& \leq\left\|L\left[U^{n}, V^{n}\right]\right\|_{1}\left\|W^{n}\right\|_{\ell_{1}}+\left\|V^{n}\right\|_{\ell_{1}} \\
& \leq(n-1) C(\Delta x)^{4}+C(\Delta x)^{4} \\
& =n C(\Delta x)^{4} .
\end{aligned}
$$

Now, for $n$ such that $0 \leq n \Delta t \leq T$, we have

$$
\left\|W^{n+1}\right\|_{\ell_{1}} \leq n C(\Delta x)^{2} \leq \frac{C T}{\Delta t}(\Delta x)^{4}=2 \nu C T(\Delta x)^{2}
$$

Hence, the method is $\mathcal{O}\left[(\Delta x)^{2}\right]$ convergent, and the finite difference scheme, Eq. C.3, provides a convergent approximation to the solution to Eq. C.1. 


\section{Bibliography}

[1] N. Ashcroft and D. Mermin, Solid State Physics, Holt-Saunders International Editions, 1976.

[2] D. Barnardin and O. E. Sero-Guillaume, "Lattice Gas Mixtures Models for Mass Diffusion", Eur. J. Mech., 8(6):1-26, 1989.

[3] R. Bellman, Introduction to Matrix Analysis, McGraw Hill Book Company, New York, 2nd ed., 1970.

[4] N. Bellomo, A. Palczewski, and G. Toscani, Mathematical Topics in Nonlinear Kinetic Theory, World Scientific, New Jersey, 1988.

[5] R. Benzi and S. SuCCI, "Bifurcations of a Lattice Gas Flow under External Forcing", J. Stat. Phys., 56(1/2):69-81, 1989.

[6] R. BenzI AND S. SUCCI, "Two-dimensional turbulence with the lattice Boltzmann equation", J. Phys. A: Math. Gen., 23:L1-L5, 1990.

[7] B. Boghosian and C. D. Levermore, "A Cellular Automaton for Burgers' Equation", Complex Systems, 1(1):17-30, February 1987. Reprinted in Lattice Gas Methods for PDEs, G. Doolen, ed., Addison-Wesley (1989):481-96.

[8] B. Boghosian and C. D. Levermore, "A Deterministic Cellular Automaton with Diffusive Behavior", in Discrete Kinetic Theory, Lattice Gas Dynamics and Foundations of Hydrodynamics, R. Monaco, ed., World Scientific, 1989, pp. 4461.

[9] B. Boghosian, W. Taylor, and D. H. Rothman, "A Cellular Automata Simulation of Two-Phase Flow on the CM-2 Connection Machine Computer", in Proceedings of Supercomputing 1988, 1988.

[10] M. Bonetti, A. Noullez, And J.-P. Boon, "Viscous Fingering in a 2-D Porous Lattice", in Discrete Kinetic Theory, Lattice Gas Dynamics, and Foundations of Hydrodynamics, R. Monaco, ed., World Scientific, 1989, pp. 395-9. 
(11] L. Brieger and E. Bonomi, "A Stochastic Cellular Automaton Simulation of the Nonlinear Diffusion Equation", in Lattice Gas Methods for PDE's: Theory, Application, and Hardware, Physica D, September 1990.

[12] C. Burges and S. Zaleski, "Buoyant Mixtures of Cellular Automata Gases", Complex Systems, 1(1):31-50, 1987.

[13] D. Burgess, F. Hayo'T, and W. F. SaAm, "Model for Surface Tension in Lattice-Gas Hydrodynamics", Phys. Rev. A, 38(7):3589-92, 1988.

[14] D. Burgess, F. Hayot, and W. F. SaAm, "Interface Fluctuations in a Lattice Gas", Phys. Rev. A, 39:4695-4700, 1989.

[15] C. Cercignani, IV. Greenberg, and P. Zweifel, "Global solution of the Boltzmann equation on a lattice", J. Stat. Phys., 20:449-62, 1979.

[16] H. Chen, S. Chen, G. D. Doolen, and W. H. Matthaeus, "A Brief Description of Lattice Gas Models for Multiphase Flows and Magnetohydrodynamics". To be published by the Sante Fe Institute, 1990.

[17] H. Chen, W. H. Matthaeus, and L. W. Klein, "An Analytic Theory and Formulation of a Local Magnetohydrodynamic Lattice Gas Model", Phys. Fluids, 32(6):1439-45, 1988.

[18] H. Chen, W. H. Matthaeus, and L. W. Klein, "Theory of Multicolor Lattice Gas: A Cellular Automaton Poisson Solver", To appear in Journal of Computational Physics, 1989.

[19] P. Chen, "GNU Emacs BiBTEX-Mode", Tech. Report 87/317, Computer Science Division, University of California, Berkeley, Berkeley, California, October 1986.

[20] P. Chen, "GNU Emacs TEX-Mode", Tech. Report 87/316, Computer Science Division, University of California, Berkeley, Berkeley, California, October 1986.

[21] P. Chen and M. A. Harrison, "Automating Index Preparation", Tech. Report 87/347, Computer Science Division, University of California, Berkeley, Berkeley, California, March 1987.

[22] P. Chen and M. A. Harrison, "Integrating Noninteractive Document Processors into an Interactive Environment", Tech. Report 87/349, Computer Science Division, University of California, Berkeley, California, April 1987.

[23] S. Chen, K. Diemer, G. D. Doolen, K. G. Eggert, and B. J. Travis, "Lattice Gas Automata for Flow Through Porous Media", in Lattice Gas Methods for PDE's: Theory, Application, and Hardware, Physica D, September 1990. 
[24] Z. Chieng, J. L, Lebowit\%, and E. R. Speer, "Microscopic Shock Structure in Model Particle Systems: The Boghosian-Levermore Cellular Automaton Revisited"; 1990. Preprint.

[25] R. C. Y. Chin, G. W, H, F, A, Howes, and J. R. MCGraw, "Parallel Computation of Multiple-scale Problems", in New Computing Environments: Parallel, Vector and Systolic, A. Wouk, ed., SIAM, Philadelphia, 1986.

[26] B. Chopard and M. Droz, "Cellular Automata Approach to Diffusion Problems", in Cellular Automata and Modeling of Complex Physical Systems, P. Manneville, N. Boccara, G. Y. Vichniac, and R. Bidaux, eds., Springer-Verlag, 1989, pp. 130-143.

[27] B. Chopard and M. Droz, "Cellular Automata Model for the Diffusion Equation", to appear in J. Stat. Phys., 1990.

[28] D. DAB AND J.-P. Boon, "Cellular Automata Approach to Reaction-Diffusion Systems", in Cellular Automata and Modeling of Complex Physical Systems, P. Manneville, N. Boccara, G. Y. Vichniac, and R. Bidaux, eds., Springer-Verlag, Berlin, 1989, pp. 257-73.

[29] T. J. Darrell, "Psfig/TeX 1.2 Users Guide", tech. report, Computer and Information Science, University of Pennsylvania, 1987.

[30] G. Doolen, ed., Complex Systems, Vol. 1, no. 4, 1987, pp. 545-851. The articles in this issue are mostly based in part on presentations given at the Workshop on Large Nonlinear Systems", held in Santa Fe, New Mexico, on October 27-29, 1986.

[31] G. Doolen, ed, Lattice Gas Methods for PDEs, Addison-Wesley, 1989.

[32] W. Eckhaus, Asymptotic Analysis of Singular Pertubations, Elsevier NorthHolland, Inc., New York, 1979.

[33] B. Elton, A. L. Perkins, And G. Rodrigue, "Matrix Substructuring, Do" main Decomposition, and Particle Methods: Current Trends for Solving PDE's in Parallel", in IEE Proceedings of the Workshop on Design and Application of Parallel Digital Processors--Lisbon, Portugal, London, May 1988, The Institution of Electrical Engineers.

[34] B. H. Elton, C. D. Levermore, and G. H. Rodrigue, "Lattice Boltzmann Methods for Some 2-D Nonlinear Diffusion Equations: Computational Results", in Asymptotic Analysis and Numerical S'olution of Partial Differentici' Equations, Marcel Dekker Inc., 1990. Proceedings of a workshop held at Argonne National 
Laboratory, Argonne, IL, February 26-28, 1990. Available as Lawrence Livermore National Laboratory Tech. Report LCRL-JC-104691.

[35] B. H. Elton and G. H, Rodrigue, "Sub-Structuring for lattice Cases", in Third International Symposium on Domain Decomposition Methods for Partial Differential Equations, Philadelphia, 1990, SIAM, pp. 451-61. Available as Lawrence Livermore National Laboratory Tech. Report UCRL 101976.

[36] U. Frisch, "Relation Between the Lattice Boltzmann Equation and the NavierStokes Equations", in Lattice Gas Methods for PDE's: Theory, Application, and Hardware, Physica D, September 1990.

[37] U. Frisch, D. D'Humières, B. Hasslacher, P. Lallemand, Y. Pomeau, AND J.-P. RIVET, "Lattice Gas Hydrodynamics in Two and Three Dimensions", Complex Systems, 1(4), August 1087.

[38] U. Frisch, B. Hasslacher, and Y. POMEaU, "Lattice Gas Automata for the Navier-Stokes Equation", Physical Review Letters, 56(14):1505-8, April 1986.

[39] M. Garby, "Quasilinear Hyperbolic-hyperbolic Singular Perturbation Problem: Study of a Shock Layer", Mathematical Methods in the Applied Sciences, 11:23752,1989 .

[40] R. Gatignol, "The Hydrodynamical Description for a Discrete Ve'ocity Model of a Gas", Complex Systems, 1:709-25, 1987.

[41] G. H. Golub and C. F. Van Loan, Matrix Computations, The John Hopkins University Press, Baltimore, Maryland, 1983.

[42] H. GraD, "Principles of the Kinetic Theory of Gases", in Handbuch der Physik, S. Flügge, ed., Vol. XIı, Springer-Verlag, Berlin, 1958, ch. 26, p. 205.

[43] H. GraD, "Theory of Rarefied Gases", in Rarefied Gas Dynamics, F. M. Devienne, ed., Vol. 3 of International Series on Aeronautical Sciences and Space Flight, Pergamon Press Inc., New York, 1960, pp. 100-138. Proceedings of the First International Symposium on Rarefied Gas Dynamics held at Nice.

[44] H. Grad, "Asymptotic Theory of the Boltzmann Equation", Physics of Fluids, 6(2):147-181, February 1963.

[45] H. GraD, "Asymptotic Theory of the Boltzmann Equation II", in Rarefied Gas Dynamics, J. A. Laurmann, ed., Vol. I of Advances in Applied Mechanics, Academic Press Inc., New York, 1963, pp. 26-59. Proceedings of the Third International Symposium on Rarefied Gas Dynamics, held at the Palais de L'unesco, Paris, in June 1962. 
[46] J. Hardy, O. DE PAzZis, AND Y, POMEAu, "Molecular dynamics of a classical lattice gas: Transport properties and time correlation functions", Physical Review A, 13(5):1949-1961, May 1076.

[47] J. HARDY AND Y, POMEAU, "Thermodynamics and Hydrodynamics for a Modeled Fluid", Journal of Mathematical Physics, 13(7):1042-1051, July 1972.

[48] J. Hardy, Y. Pomeau, and O, DE Pazzis, "Time evolution of a twodimensional model system. I. Invariant states and time correlation functions", Journal of Mathematical Physics, 14(12):1746-59, December 1973.

[49] F. J. Higuera, S. Succi, and R. Benzi, "Lattice Gas Dynamics with Enhanced Collisions", Europhysics Letters, 9(4):345-9, June 1989.

[50] P. G. Hoel, S. C. Port, and C. J. Strone, Introduction to Stochastic Processes, Haughton Mifflin Company, Boston, Massachusetts, 1972.

[51] L. Lamport, $\left[a T_{E} X\right.$ : A Document Preparation System, Addison-Wesley Publishing Company, 1985.

[52] Lattice Gas Methods for PDE's: Theory, Application, and Hardware, Physica D, September 1990. Proceedings of a NATO-sponsored workshop held at Los Alamos :'ational Laboratory, September 1989.

[53] J. L. Lebowitz, E. Orlandi, and E. Presutti, "Convergence of Stochastic Cellular Automaton to Burgers' Equation: Fluctuations and Stability", Physica D, 33:165-1's8, October/November 1988.

[54] C. D. Levermore. Private communication.

[55] J. E. Marsden, Elementary Classical Analysis, W. H. Freeman and Company, San Francisco, 1974.

[56] G. Mcnamara and G. Zanett, "Using the Lattice Boltzmann Equation to Simulate Lattice Gas Automata", Physical Review Letters, 61(20), 1988.

[57] R. Monaco, ed, Discrete Kinetic Theory, Lattice Gas Dynamics and Foundations of Hydrodynamics, World Scientific, 1989. Proceedings of a workshop on the subject of the title (above) held in Torino, Italy, Sept. 20-4, 1988.

[58] D. Montgomery and G. D. Doolen, "Two Cellular Automata for Plasma Computations", Complex Systems, 1:831-8, 1987. Reprinced in Lattice Gius Methods for PDEs, G. Doolen, ed., Addison-Wesley (1989):461-70. 
[59] A. Palczewski, "Boltzmann Equation on a lattice: global solution for nonMaxwellian gases", Arch. Mech., 34:287-96, 1082.

(60) A. L. Perkins and G. Rodrigue, "A Domain Decomposition Method for Solving a 2-Dimensional Viscous Burgers' Equation", 'T'ech. Report UCRL-09823 Rev. 1, Lawrence Livermore National Laboratory, 1989. To appear in Applied Numerical Mathematics.

[61] L. Perkins, Parallel Heterogeneous Mesh Refincment For Multidimensional Convection-Diffusion Equations Using An Euler-Lagrange Method, Ph.D. Dissertation, University of California, Davis, CA, June 1989. Available as Lawrence Livermore National Laboratory report \#UCRL-53950.

[62] V. L. Peterson, "Computational Challenges in Aerospace", Future Generation Computer Systems, 5(2-3):243-258, 1989.

[63] M. H. Protter and H. F. Weinberger, Maximum Principles in Differential Equations, Springer-Verlag, 2nd ed., 1984.

[64] F. REIF, Fundamentals of statistical and thermal physics, McGraw-Hill, 1965.

[65] C. E. Rhoades, ed., Future Generation Computer Systems, Vol. 5, nos. 2-3, September 1989, pp. 167-350. Special Issue on "Grand Challenges to Computationai Science".

[66] R. D. Richtmyer and K. W. Mor'Ton, Difference Methods for Initicl-Value Problems, Vol. 4 of Interscience Tracts in Pure and Applied Mathematics, Interscience Publishers (John Wiley \& Sons), 2nd ed., 1967.

[67] H. A. Scilwarrz, "Ueber einen Grenzübersang durch alternirendes Verfahren", in Gesammelte Mathemaissche Abhandlungen, Vol. 2, Springer Verlag, Berlin, 1890. Translation available as Lawrence Livermore National Laboratory Tech. Report UCRL TRANS-11893, August 1983.

[68] J. S. Scroggs, The S'olution of a Parabolic Partial Differential Equation via Domain Decomposition: The Synthesis of Asymptotic and Numerical Analysis, Ph.D. Dissertation, University of Illinois, Urbana, IL, May 1988.

[69] G. A. SoD, Numerical Methods in Fluid Dynamics: Initial and Initial BoundaryValue Problems, Cambridge University Press, Cambridge, 1988.

[70] J. C. Strukwerda, Finite Difference Schemes and Partial Differential Equations, Wadsworth \& Brooks/Cole Advanced Books \& Software, Pacific Grove, California, 1989. 
[71] G. B. Wur'run M, Linear and Nonlinear Waves, Pure and Applied Mathematics: A Wiley-Interscience Series of 'lexts, Monographs, and Tracts, John Wiley \& Sons, 1.974.

[72] S. Wolrmam, "Cellular Automaton Fluids 1: Basic 'Theory", Journal of S'tatistical Physics, 45(3/4):171-526, November 1986. 


\section{Index}

A boldface page number, e.g.. 21, indicates the location of a definition.

advection bias, 44, 49,155, 181,210, 215,216

advection operator, xiv, $19,28,45$ asymptotic expansion, 66 derivative operations in, 62 Taylor expansion, 45, 66 advection operator expansion, 62,64 , 67

asymptic expansion, 44,45

of advection operator, 66

of coilision operator, 61

o. $\mathrm{n}, 47$

Bravais lattice, 15

Burgers' Equation, 5, 96, 121, 154, $176,180,181,209,231,232$,

245

LB method for, 154, 195, 209

maximurn/minimum principle for, 176

cellular automata, iii, 2

cellular fluids, 2

Chapman-Enskog consistency condition, see consistency condition

Chapman-Enskog-Taylor asymptotic equilibrium expansion, 61

circulant, 50, 52, 188

collision operator, xiv, $\mathbf{2 8}, 83$

linearized, see linearized collision operator of LB method for Burgers' equation, 155

ordering of, 32

Taylor expansion, 45, 58, 61

without Fermi Exclusion Principle, 34

collision operator expansion, 58,67

collision rules, 14, 19, 21ff, 121, 155

compatibility condition, see consistency condition

componentwise product $(\star), x \mathrm{iii}, 9$, $48,54,55,58$

using, 57

conditions of monotonicity, $\mathbf{8 5}, 96$, 97

connectedness, 12

conservative form, 246

conserved quantity, 21

consistency, $83, \mathbf{8 5}, 88 \mathrm{ff}, 117$

in determining convergence, 115

theorem on, 89

consistency condition, 46-48, 1, 72, 83,88

free parameters, 46

hydrodynamical equation, 71

continuum maximum/minimum principle, see maximum/minimum principle

convergence, $82 \mathrm{ff}, \mathbf{8 8}, 97,118,122$ 
computational evidence, 195-220

general results, 83

general theorem, 115

proof of for a nonlinear differ-

ence method, 245-252

covariance, 13, 33, 157, 185

in LB method for Burgers' equa-

tion, 121

detail balance, 24

in determining equilibrium solutions, 24

deterministic lattice gas method, 29, sce lattice gas method

differentials, 10

direct method, 158

discrete Chapman-Enskog analysis, $44 \mathrm{ff}$

discrete Chapman-Enskog expansion, $48,55,71,89,122$

and consistency, 88

equilibrium expansion, 47

discrete Chapman-Enskog procedure, 7,48

discrete maximum/minimum principle, see maximum/minimum principle

domain decomposition for lattice methods, $221 \mathrm{ff}$

domain of monotonicity, 83, 86, 97

in stability, 113

eigenmatrix, $\mathbf{5 2}$

$\vec{e}_{k}$, see velocity vectors

ensemble average, 13

equilibria, 7

in $L B_{1}$ via direct method, 36

in $\mathrm{LB}_{1}$ via equilibrium theorem, 42

via direct method, 36

via Equilibrium Theorem, 36 equilibrium, 35

equilibrium analysis, 35

equilibrium expansion, xiv, 44, 47, $62,66,70$

equilibrium function, 44

equilibrium theorem, 36

applying it, 41

ergodicity, 14

error

in truncated equilbrium expansion, 87

extreme points

of domain of monotonicity, 87 , 97

Fermi Exclusion Principle, 14, 34, $35,49,232$

example of lattice Boltzmann

method without the, 34

grid, see lattice

ground state, 44

$H, \mathbf{8 5}, 96$

hydrodynamical equation, 4!, 71, 83

hydrodynamical error, 88, 117

hydrodynamical mode, 87

hydrodynamical modes, 83

Jacobian matrix, 8, 11

$\mathfrak{L}$, see Bravais lattice

$\mathcal{L}$, see linearized collision operator

$\mathcal{L}^{+}$, see pseudo-inverse

$L[\mathbf{n}, \mathbf{h}], \mathbf{8 7}$

lattice, see Bravais lattice

Lattice Boltzmann Approximation, iv, 7, 32, 33, 118, 231

ir discrete Chapman-Enskog expansion, 44

$\mathrm{LB}_{2}, 125$ 
lattice Boltzmann collision operator, 32 , see also collision operator Lattice Boltzmann Equation, 32, 33, $35,46,48,85-87,89,95,97$, 138

lattice Boltzmann method, iv, $\mathbf{3 3}$ without Fermi Exclusion Principle, 34 example of, 34

lattice gas automata, 2

lattice gas method, 28

lattice gas methods, $14 \mathrm{ff}$

lattice isometry invariance, 21

lattice node, 15

LB method, see lattice Boltzmann method

LB $\mathrm{B}$ method for 2-D advection-diffusion, 195

advection bias, 181

collision operator, 181

collision rules, $181 \cdots 18 \%$

componentwise product, 188

computational results, 214-220

consistency condition

$\mathcal{O}\left[\delta^{2}\right], 190$

$\mathcal{O}\left[\delta^{3}\right], 192$

covariance, 185

discrete Chapman-Enskog expan-

sion, 188-192

equilibria, 186

hydrodynamical equation, 192

lattice, 181

Lattice Boltzmann Approxima-

tion, 185

velocity vectors, 181,189

LB method for Burgers' equation, 195

advection bias, 155

advection operator, 158

collision operator, 155 collision rules, 155,156

componentwise product, 160

computational results, 209-213

consistency, 170

consistency condition

$\mathcal{O}\left[\delta^{2}\right], 163$

$\mathcal{O}\left[\delta^{3}\right], 165$

$\mathcal{O}\left[\delta^{4}\right], 167$

$\mathcal{O}\left[\delta^{5}\right], 169$

continuum maximum/minimum principle, 176

convergence, $168,170,178,180$

covariance, 157,180

discrete Chapman-linskog expan-

sion, 160-170, 172, 173

discrete maximum/minimum

principle, 175

domain decomposition, 223

domain of monotonicity, 174

extreme values of, 175

equilibria, 158

H, 174

Lattice Boltzmann Approximation, 157

Lattice Boltzmann Equation, 158, 174

stability, 178

truncated equilibrium expansion, 170

truncation error, 171

$L^{L} B_{1}, 195$

advection operator

derivatives in, 63

circulant $\mathcal{L}, 51$

collision operator, 29,30

collision operator expansion, 81

collision rules, $21-23,31$

componentwise product, 55,69 , 70

componentwise product $(\star), 55$ 
computational results, $197-202$

consistency, 90

consistency condition

form of, 71

$\mathcal{O}\left[\delta^{2}\right], 75$

$\mathcal{O}\left[\delta^{3}\right], 77$

$\mathcal{O}\left[\delta^{4}\right], 78$

$\mathcal{O}\left[\delta^{5}\right], 81$

coitinuum maximum/minimum

principle, 110,111

convergence, 117

detailed balance, 24, 42

direction labels, 17

discrete Chapman-Enskog expan-

sion, 72-81, 94

discrete maximum/minimum

principle, 108

equilibria, 36, 42, 43

equilibrium solution, 47

ground state, 47

H, 99

hydrodynamical equation, 111

lattice, 17

Lattice Boltzmann Approxima-

tion, 33

lattice Boltzmann collision op-

erator, 33

Lattice Boltzmann Equation, 99

linearized collision operator, 50

eigenpairs of, 52

nullspace, 53

pseudo-inverse, 54

rotation invariance, 31,101

simplifying gradient expressions, 69,70

stability, 114

truncated equilibrium expansion, $72,90,91,93$

truncation error, 93

velocity vectors, 17
$L B_{2}, 195$

analysis, $122-154$

collision ope;ator, 125

collision rules, 122-124

componentwise product, 128

computational results, 203-208

consistency, 137, 153

consistency condition, 154

$\mathcal{O}\left[\delta^{2}\right], 131$

$\mathcal{O}\left[\delta^{3}\right], 133$

$\mathcal{O}\left[\delta^{4}\right], 135$

$\mathcal{O}\left[\delta^{5}\right], 137$

continuum maximum/minimum

principle, $150,152,154$

convergence, $137 \mathrm{ff}, 153$

detailed balance, 125

discrete Chapman-Enskog expan-

sion, 127-137, 141

discrete maximum/minimum

principle, 150

domain of monotonicity, 141

equilibria, 125

equilibrium solution, 127

H, 142

hydrodynamical error, 153

lattice, 122

Lattice Boltzmann Equation, 142

linearized collision operator, 127

stability, 152

truncated equilibrium expansion, 137, 153

truncation error, 140

velocity vectors, 122

LG method, see lattice gas method linearized collision operat,or, 46, 49, 55,83

and (semi-)detailed balance, 24

and quasi-detailed balance, 28

in matched expansion analysis, 46 
in terms of iis eigenpairs, 53

mass conservation, $\mathbf{2 4}, 83,113,114$

maximum principle

continuum, 246

discrete, 247

maximum/minimum principle, $83,95 \mathrm{ff}$

continuum, 97, 110, 111

discrete, $95-97$

general theorem, 97

for Burgers' equation, 176

mean occupation number, $\mathbf{3 1}$

microdynamical equation, $\mathbf{1 8 f f}, 33$

for mean occupation numbers, 31

microdynamical evolution equation, see microdynamical equation

molecular chaos, 14, 125

norms, 11

$\mathcal{O}[\cdot]$, xiii, 12

occupation number, 16

occupation numbers, 44

vector of, 19

without Fermi Exclusion Prin-

ciple, 35

particle speed, 15

permutation matrix, 116

probabilistic lattice gas method, 29, see lattice gas method

pseudo-inverse $\left(\mathcal{L}^{+}\right), 53$

quasi-detailed balance, $\mathbf{2 5}$

reflection invariance, 50

rotation invariance, 29

semi-detailed balance, 25,47

in determining equilibrium solutions, 24

set volume, 12 spatial-gradient expansion, 44

stability, 83, 87, 113ff in determining convergence, 115 in $L_{1}$-norm, 113

state, 19, see also occupation numbers

state transition function, 19, 20ff

stochastic lattice gas method, 29, see lattice gas method

su's-structuring for lattice methods, $221 \mathrm{ff}$

Taylor expansion, 46

of adyection operator, 45, see also advection operator, 62,66

of collision operator, 45,58 , see also collision operator

Taylor series expansion of vector function, 63

truncated equilibrium expansion, 82 , $\mathbf{8 5}, 87 \cdots 89,91,97,117$

in consistency, 85

truncation error, 85

unit velocity vector, see velocity vectors

vector exponentiation, 10

velocity vectors, $\mathbf{1 5}, 19,62,65,128$ 

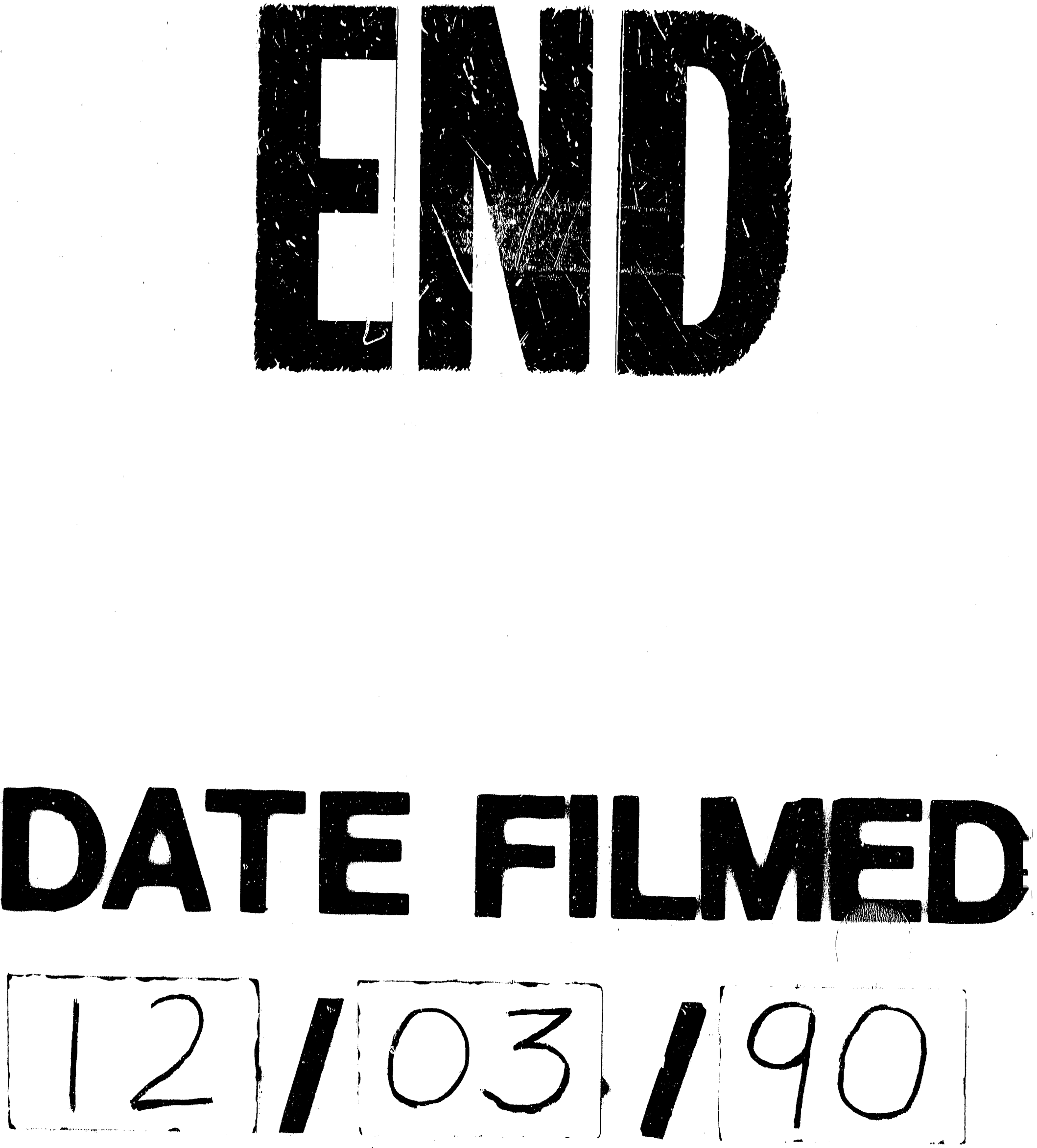
